LBNL-43546

\title{
I. Advances in NMR Signal Processing II. Spin Dynamics in Quantum Dissipative Systems
}

\author{
Yung-Ya Lin \\ Ph.D. Thesis \\ Department of Chemistry \\ University of California, Berkeley \\ and \\ Materials Sciences Division \\ Ernest Orlando Lawrence Berkeley National Laboratory \\ University of California \\ Berkeley, CA 94720
}

November 1998

This work was supported by the Director, Office of Energy Research, Office of Basic Energy Sciences,

Materials Sciences Division, of the U.S. Department of Energy under Contract No. DE-AC03-76SF00098. 


\section{DISCLAIMER}

This report was prepared as an account of work sponsored by an agency of the United States Government. Neither the United States Government nor any agency thereof, nor any of their employees, make any warranty, express or implied, or assumes any legal liability or responsibility for the accuracy, completeness, or usefulness of any information, apparatus, product, or process disclosed, or represents that its use would not infringe privately owned rights. Reference herein to any specific commercial product, process, or service by trade name, trademark, manufacturer, or otherwise does not necessarily constitute or imply its endorsement, recommendation, or favoring by the United States Government or any agency thereof. The views and opinions of authors expressed herein do not necessarily state or reflect those of the United States Government or any agency thereof. 


\section{DISCLAIMER}

Portions of this document may be illegible in electronic image products. Images are produced from the best available original document. 


\section{Advances in NMR Signal Processing II. Spin Dynamics in Quantum Dissipative Systems by Yung-Ya Lin}

B.A. (National Taiwan University) 1990
M.S. (National Taiwan University) 1993

A dissertation submitted in partial satisfaction of the requirements for the degree of

Doctor of Philosophy

in

Chemistry

in the

GRADUATE DIVISION

of the

UNIVERSITY of CALIFORNIA at BERKELEY

Committee in charge:

Professor Alexander Pines, Chair

Professor Daniel M. Neumark

Professor Jeffrey A. Reimer

Fall 1998 


\title{
I. Advances in NMR Signal Processing II. Spin Dynamics in Quantum Dissipative Systems
}

\author{
Copyright $@ 1998$
}

by

Yung-Ya Lin

The U.S. Department of Energy has the right to use this document for any purpose whatsoever including the right to reproduce all or any part thereof. 


\author{
Abstract \\ I. Advances in NMR Signal Processing \\ II. Spin Dynamics in Quantum Dissipative Systems \\ by \\ Yung-Ya Lin \\ Doctor of Philosophy in Chemistry \\ University of California at Berkeley \\ Professor Alexander Pines, Chair
}

Part I. Advances in NMR Signal Processing. Improvements of sensitivity and resolution are two major objects in the development of NMR/MRI. A signal enhancement method is first presented which recovers signal frorıi noise by a judicious combination of a prioriknowledge to define the desired feasible solutions and a set theoretic estimation for restoring signal properties that have been lost due to noise contamination. The effect of noise can be significantly mitigated through the process of iteratively modifying the noisy data set to the smallest degree necessary so that it possesses a collection of prescribed properties and also lies closest to the original data set. A novel detection-estimation scheme is then introduced to analyze noisy and/or 
strongly damped or truncated FIDs. Based on exponential modeling, the number of signals is detected based on information theory and the spectral parameters are estimated using the matrix pencil method.

Part II. Spin Dynamics in Quantum Dissipative Systems. Spin dynamics in manybody dipole-coupled systems constitutes one of the most fundamental problems in magnetic resonance and condensed-matter physics. Its many-spin nature precludes any rigorous treatment. Therefore, the spin-boson model is adopted to describe in the rotating frame the influence of the dipolar local fields on a tagged spin. Based on the polaronic transform and a perturbation treatment, an analytical solution is derived, suggesting the existence of self-trapped states in the strong coupling limit, i.e., when transverse local field $\gg$ longitudinal local field. Such nonlinear phenomena originate from the joint action of the lattice fluctuations and the reaction field. Under semiclassical approximation, it is found that the main effect of the reaction field is the renormalization of the Hamiltonian of interest. Its direct consequence is the two-step relaxation process: the spin is initially localized in a quasiequilibrium state, which is later detrapped by the lattice fluctuations in an extended time scale. Lowtemperature measurements and classical-spin simulations are carried out to verify the above analysis.

To promote the implementation and future study on the topics described in this thesis, program packages of advanced NMR signal processing and many-spin FID simulations are summarized and listed in the Appendix. 
To my parents

and

Nien-Hui 


\section{Contents}

List of Figures vii

List of Tables $\quad$ ix

I Advances in NMR Signal Processing 1

1 Set Theoretic Estimation: Theory 3

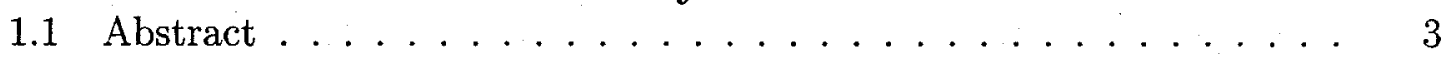

1.2 Introduction . . . . . . . . . . . . . . . 4

1.3 Best Feasible Approximation . . . . . . . . . . . . . . . . 5

1.4 Monte Carlo Simulations . . . . . . . . . . . . . . . . . 8

1.5 Conclusion ............................. 14

$\begin{array}{ll}\text { Bibliography } & 18\end{array}$

2 Set Theoretic Estimation: Algorithm 21

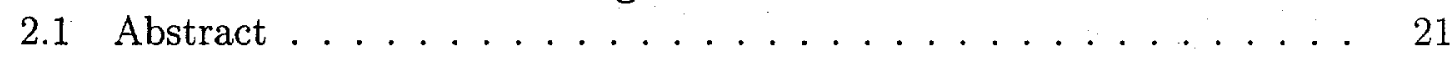

2.2 Introduction . . . . . . . . . . . . . . . . 22

2.3 Iterative Parallel Projections . . . . . . . . . . . . . . . 23

2.4 Sensitivity Enhancement in ${ }^{43} \mathrm{Ca}$ NMR . . . . . . . . . . . . . 25

2.5 Resolution Enhancement in ${ }^{25} \mathrm{Mg}$ NMR . . . . . . . . . . . . . . . . . . . . . . . .

2.6 Conclusion . . . . . . . . . . . . . . . . 38

Bibliography $\quad 41$

3 Matrix Pencil 44

3.1 Abstract ........................... 44

3.2 Introduction . . . . . . . . . . . . . . 45

3.3 Matrix Pencil Method . . . . . . . . . . . . . . . . 49

3.4 Information Theory . . . . . . . . . . . 57 
3.5 Monte Carlo Sinnulations . . . . . . . . . . . . . . . . . . 59

3.6 Experimental Applications . . . . . . . . . . . . . . 74

3.7 Conclusion . . . . . . . . . . . . . . . . 77

$\begin{array}{ll}\text { Bibliography } & 80\end{array}$

II Spin Dynamics in Quantum Dissipative Systems $\quad 87$

4 Solitonic Spin Dynamics $\quad 89$

4.1 Abstract . . . . . . . . . . . . . . . . . . . . 89

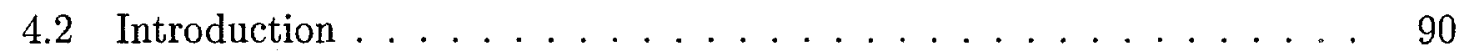

4.3 Experimental Verification $\ldots \ldots \ldots \ldots . \ldots . \ldots . \ldots 9$

4.4 Spin-Boson Hamiltonian . . . . . . . . . . . . . . . . 95

4.5 Reaction Field . . . . . . . . . . . . . . . . . . 97

4.6 Solitonic Modes . . . . . . . . . . . . . . . . . . . . . 100

4.7 Conclusion . . . . . . . . . . . . . . . . . . . . 101

$\begin{array}{ll}\text { Bibliography } & 105\end{array}$

5 Spin-Boson Hamiltonian 107

5.1 . Abstract . . . . . . . . . . . . . . . . . . . . 107

5.2 Introduction . . . . . . . . . . . . . . . 108

5.3 Polaronic Transformation . . . . . . . . . . . . . . . . . . . . 110

5.4 Second-Order Perturbation . . . . . . . . . . . . . . . . 114

5.5 Mean-Field Approximation . . . . . . . . . . . . 117

5.6 Trapped States . . . . . . . . . . . . . . . . . . . . . . . . . 123

5.7 Two-Dimensional Spin-Boson Hamiltonian . . . . . . . . . . . 126

5.8 Conclusion . . . . . . . . . . . . . . . . . . . . . . . 134

$\begin{array}{ll}\text { Bibliography } & 135\end{array}$

6 Hamiltonian Renormalization $\quad 137$

6.1 Abstract . . . . . . . . . . . . . . . . 137

6.2 Introduction . . . . . . . . . . . . . . . . . . 138

6.3 Semiclassical Approximation . . . . . . . . . . . . . . . . . . 139

6.4 Fokker-Planck Equation . . . . . . . . . . . . . . . . . . . 143

6.5 Numerical Simulation . . . . . . . . . . . . . . . . . . 147

6.6 Deterministic Chaos . . . . . . . . . . . . . . . 153

6.7 Conclusion . . . . . . . . . . . . . . . . . . . 154

$\begin{array}{ll}\text { Bibliography } & 158\end{array}$ 
7 Classical Spin Simulations 159

7.1 Abstract . . . . . . . . . . . . . . . . . . . . 159

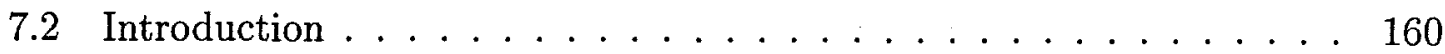

7.3 Computational Procedures . . . . . . . . . . . . . . . . . 163

7.4 Simulation Results . . . . . . . . . . . . . . . . . 165

$\begin{array}{ll}\text { Bibliography } & 172\end{array}$

$\begin{array}{ll}\text { III Appendix } & 173\end{array}$

A Advanced NMR Signal Processing Package $\quad 175$

A.1 Matrix Pencil . . . . . . . . . . . . . . . 176

A.1.1 Matrix Pencil Method with Information Theoretic Criteria . . 178

A.1.2 Linear Prediction with Singular Value Decomposition . . . . . 180

A.2 Set Theoretic Estimation . . . . . . . . . . . . . . . 182

A.2.1 B. de Moor algorithm . . . . . . . . . . . . . . . . . . 183

A.2.2 Iterative Parallel Projections . . . . . . . . . . . . . . 185

A.3 System Identification . . . . . . . . . . . . . . . . . . . . 194

A.3.1 Padé Approximation . . . . . . . . . . . . . . . . . . 194

A.3.2 Prony Method . . . . . . . . . . . . . . . . . . 196

A.3.3 Iterative Prefiltering . . . . . . . . . . . . . . . 198

A.3.4 Autocorrelation Method . . . . . . . . . . . . . . . . 199

A.3.5 Covariance Method . . . . . . . . . . . . . . . . . . . 201

A.4 Spectral Estimation . . . . . . . . . . . . . . . . . . . 202

A.4.1 Maximum Likelihood Method . . . . . . . . . . . . 203

A.4.2 Maximum Entropy Method . . . . . . . . . . . . . 204

A.4.3 Noise Subspace Methods . . . . . . . . . . . . . . . . 205

A.4.4 Signal Subspace Method . . . . . . . . . . . . . . . . . 210

$\begin{array}{ll}\text { Bibliography } & 212\end{array}$

B Many-Spin FID Simulation Package 215

B.1 Quantum Spins . . . . . . . . . . . . . . . . . . . 215

B.2 Classical Spins . . . . . . . . . . . . . . . . 221

B.2.1 Dipole-Dipole Interaction . . . . . . . . . . . . 225

B.2.2 Dipolar Local Fields . . . . . . . . . . . . . . . . . . . . 227

B.2.3 Equation of Motion . . . . . . . . . . . . . . . . . 228

B.2.4 Initial Configurations . . . . . . . . . . . . . . 229

B.2.5 Pulse Rotation . . . . . . . . . . . . . . . . . 232

B.2.6 Canonical Ensemble Average . . . . . . . . . . . . . 233

$\begin{array}{ll}\text { Bibliography } & 235\end{array}$ 


\section{List of Figures}

1.1 Set theoretic estimation: feasibility set . . . . . . . . . . 6

1.2 Best feasible approximation . . . . . . . . . . . . . . 9

1.3 Statistical results of the Monte Carlo simulations with and without signal-enhancement preprocessing: bias and standard deviation . . . .

1.4 Statistical results of the Monte Carlo simulations with and without signal-enhancement preprocessing: success rate . . . . . . . . . 16

2.1 Sensitivity enhancement in ${ }^{43} \mathrm{Ca}$ NMR $\ldots \ldots \ldots . \ldots . \ldots 32$

2.2 Algorithm convergence of the iterative parallel projections . . . . . 33

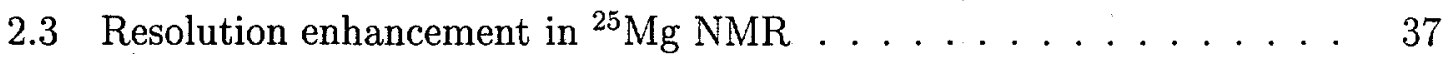

2.4 Rank determination by the relative amplitudes of the singular values 39

3.1 Flowchart of the ITMPM algorithm . . . . . . . . . . . . 60

$3.2{ }^{19} \mathrm{~F}$ spectrum of p-fluorophenol . . . . . . . . . . . . 67

3.3 Statistical results of the Monte Carlo simulations . . . . . . . . . . 69

$3.4{ }^{1} \mathrm{H}$ spectra of glutamic acid in $\mathrm{D}_{2} \mathrm{O} \ldots \ldots \ldots . \ldots 72$

3.5 Functional values of MDL criterion v.s. singular values . . . . . . . 73

$3.6{ }^{1} \mathrm{H}$ spectra of static polycrystalline anthracene and malonic acid . . . 76

3.7 Spectra of an aqueous solution containing $0.20 \mathrm{M} \mathrm{CaCl}_{2}$ and $0.10 \mathrm{M}$ sodium EDTA ...................... 78

$4.1{ }^{1} \mathrm{H}$ spectra of static polycrystalline glycine at $4 \mathrm{~K} \ldots \ldots . \ldots 93$

$4.2{ }^{1} \mathrm{H}$ spectra of static polycrystalline alanine at $4 \mathrm{~K} \ldots \ldots . \ldots 9$

4.3 FID $\left\langle I_{x}(t)\right\rangle$ and reaction field $\left\langle\tilde{\omega}_{x}^{(R)}(t)\right\rangle$ as a function of longitudinal

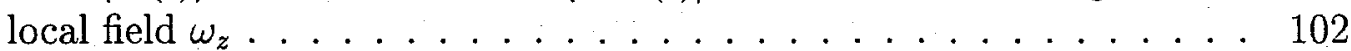

4.4 FID $\left\langle I_{x}(t)\right\rangle$ and reaction field $\left\langle\tilde{\omega}_{x}^{(R)}(t)\right\rangle$ as a function of spin-lattice coupling strength $\lambda \ldots \ldots \ldots \ldots$

5.1 FID $\left\langle I_{x}(t)\right\rangle$ and reaction field $\left\langle\tilde{\omega}_{x}^{(R)}(t)\right\rangle$ as a function of dipolar order $\alpha 125$ 
viii

6.1 A typical bistable trajectory of the spin-boson Hamiltonian under semiclassical approximation . . . . . . . . . . . . . . . . 148

6.2 Self-trapping of a tagged classical spin under dipolar local fields . . . 152

6.3 Chaotic trajectories of a tagged classical spin under diolar local fields: effect of numerical precision . . . . . . . . . . . . . . . . 155

6.4 Chaotic trajectories of a tagged classical spin under diolar local fields: effect of small tipping angle . . . . . . . . . . . . . . . . 156

6.5 Chaotic trajectories of a tagged classical spin under diolar local fields: effect of large tipping angle . . . . . . . . . . . . . . . 157

7.1 FID of a tagged classical spin with and without reaction field . . . . . 167

7.2 Long-time tail as a function of spin-lattice coupling strength . . . . . 168

7.3 Typical trajectories of a tagged classical spin with and without reaction

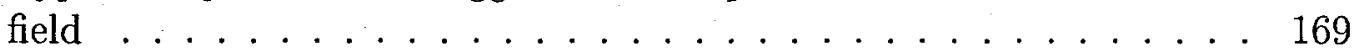

7.4 FID of a tagged classical spin as a function of dipolar order $\alpha \ldots 171$ 


\section{List of Tables}

3.1 Bias \pm Standard Deviation of the Estimated Spectral Parameters . . 63

7.1 New Trajectories Based on Symmetry Considerations $(i=0, \cdots, N-1) 165$

A.1 Summary of Advanced NMR Signal Processing Package . . . . . . . 177

B.1 Summary of Subroutines for Classical Spin Simulations . . . . . . . . 221 


\section{Introduction}

Part I. Advances in NMR Signal Processing.

Within the last decade, there has been a flurry of research activity into formulating and comparing alternative means of NMR signal processing. The primary motivation has apparently been the development of techniques for accurate spectral quantification and enhanced spectral resolution. In Chapter 1, a mathematical signal enhancement method is presented, which seeks to reduce the degradation made on the excitationresponse observations and to enhance the spectral/image estimators' performance. This object is achieved by using a wide range of $a$ priori knowledge available from our theoretical and experimental understanding of the underlying spin system to define the desired signal, and a set theoretic estimate to restore signal properties that have been lost due to noise corruption and measurement distortion. Among various novel algorithms, the feasibility algorithm of iterative parallel projections demonstrates certain theoretical and computational advantages for practical NMR applications, as demonstrated in Chapter 2. It is shown via Monte Carlo simulations and experiments that a significant enhancement in spectral sensitivity and resolution can be achieved by applying this method as a preprocessing step prior to routine data analysis, at the expense of greater computational complexity.

Many potentially interesting and useful classes of NMR experiments generate data for which conventional spectral estimation and quantification via the Fourier Transform is unsatisfactory. In particular, recently introduced solid-state NMR experi- 
ments which involve long delays before data acquisition (discussed in Part II) fall into this category, as the free induction decays are heavily "truncated" and have low signal-to-noise ratios. A novel detection-estimation scheme is introduced in Chapter 3 in order to analyze data from such experiments and others where the sensitivity is low and/or the data record is strongly damped or truncated. This method not only improves the estimation accuracy (by a factor of 2-4) with a lower "breakdown" signal-to-noise threshold $(\approx 1.5 \mathrm{~dB})$, but also reduces the computational cost by about an order of magnitude. It also holds great promise in effectively reducing truncation artifacts.

One of the most effective ways to gain a deep appreciation and understanding of such advanced techniques is to process NMR signals. Therefore, to assist in the implementation of these advanced NMR signal processing techniques, a package of MATLAB programs is constructed, as documented in Appendix A. None of these programs in this package are particularly long and may be typed-in by hand without too much difficulty.

Part II. Spin Dynamics in Quantum Dissipative Systems.

For abundant spins in solids, particularly those with high gyromagnetic ratios, the homonuclear dipolar coupling is the dominant internal interaction. Spin dynamics in such systems, as reflected in the measured free induction decay, is a subject that has been of great theoretical and experimental interest since the birth of magnetic resonance. The understanding is crucial to the interpretation of experimental results 
xii

and the development of new methodologies in NMR and related techniques, let alone its fundamental importance in quantum and statistical mechanics. In Chapter 4, the existence of the solitonic modes in long-time evolution is experimentally confirmed and theoretically modeled by a two-dimensional spin-boson Hamiltonian to describe the influence of the fluctuating dipolar local-fields upon the quantum coherence in connection with the macroscopic observable. Such striking phenomena, totally unexpected within current NMR theoretical framework, originate from the nonlinearity triggered by the joint action of the lattice fluctuations and the reaction field: the spins fluctuate and polarize the lattice which reacts back on the spins. The lattice fluctuations are not independent of the spin states, and the crucial problem is precisely how to take this effect into account. As shown in Chapter 5, our simple perturbation treatment based on the two-dimensional spin-boson Hamiltonian supplements the prototype approaches with proper inclusion of the reaction field in addition to the direct field in describing the dynamics of the spins coupled to a dissipative lattice.

We then illustrate in Chapter 6 how to carry out a semiclassical analysis without losing the influence of the reaction field. The semiclassical approximation allows us to simplify the analysis by replacing the quantum-mechanical dissipation process with the standard classical fluctuation-dissipation process. The resulting unusual spin relaxation process can then be satisfactorily accounted by a renormalization of the Hamiltonian of interest. Initially, the spin is localized in a self-trapped state due to the similar mechanism of Davydov soliton. The lattice fluctuations eventually 
provoke the destruction of this localized state with an extended time scale, in a way similar to the thermal instability of the Davydov soliton at physiological temperature. It is also demonstrated that the trajectories of classical spins show signs of chaos: sensitivity nonlinearly amplifies even the smallest difference in the initial conditions. Both the self-trapped states and the chaotic trajectories are again provoked by the reaction field. Molecular-dynamics simulation for classical spins in a rigid lattice is carried out in Chapter 7 to test the validity of the above analysis. When the spin-lattice coupling strength and/or the dipolar order is increased, the resulting solitonic dynamics is expected to become even more pronounced. To promote user's implementation and future study, a concise GAMMA program for quantum spins is documented in Appendix B, which simulates the FID of a polycrystalline dipolecoupled many-spin system with or without magic-angle spinning. A collection of MATLAB m-files for classical-spin FID simulations then follows. 
xiv

\section{Acknowledgements}

I want to thank my advisor, Professor Alexander Pines, for creating an exciting research environment, and providing me with the freedom to pursue our own ideas as well as the invaluable guidance and support at various pivotal points of my graduate career. His enthusiasm infects the group so that excellence and originality in research work is encouraged and expected. Even more important, his indefatigable good humor, scientific sense, and tolerance for my peculiarities have kept our scientific endeavors marching forward and our personal relationship warm. I consider him a friend.

Our group administrative assistant and mother to us all, Dione Carmichael, has been invaluable in the day-to-day operations of the group as well as always offering her guidance and encouragement. From her, I realize that in front of every great professor is an amazing Secretary. The assistance from Dr. Gerry Chingas, both at technical and scientific levels, is highly appreciated.

Alex has attracted outstanding graduate students and postdocs, and has created a group which is intellectually stimulating and hardworking, yet is relaxed and friendly. I consider it to be an impressive accomplishment. In a short space and in such a large group, it is impossible for me to properly thank all the pinenuts who have helped me in one way or another. Hence it must suffice to list a few names and extend my gratitude to all the others. I have been privileged to work with Dr. Matthias Ernst, Dr. Paul Hodgkinson, Dr. Jeffrey Yarger, Dr. Shuanhu Wang, Dr. Nien-Hui Ge, Dr. 
Young Lee and Jamie Walls. There have been many others in the group that I did not work with directly, but who supported me in many ways and made the group such an enjoyable working environment. Among them, special thanks are due to Dr. Roberto Seydoux, Dr. Matthew Augustine, Dr. Marco Tomaselli, Dr. Yang Li, Bin $\mathrm{He}$, and Seth Bush. It is the sort of vibrant intellectual environment I doubt could be recreated in many other locations.

I have to thank Dr. Geoff Barrell, Dr. Sheryl Gann, David deGraw, Dr. Jeffrey Yarger, Dr. Marco Tomaselli and Robert Havlin. They selflessly manage the group's computing resources. I have also benefited from insightful and interesting discussions with Prof. David Chandler, Prof. Erwin Hahn and Prof. Jean Jeener.

I want to thank my family for their love and support over all the years. My parents have always encouraged me in all I did and provided me with the best education possible. I can not thank them enough for all they have given me. For almost eleven years, my wife, Nien-Hui, and I have gone through the challenge in college and graduate school together. Without her love and support, I would not have been able to accomplish this work.

This work was supported by the Director, Office of Energy Research, Office of Basic Energy Sciences, Materials Sciences Division, U.S. Department of Energy, under contract No. DE-AC03-76SF00098. 
Part I

Advances in NMR Signal

Processing 



\section{Chapter 1}

\section{Set Theoretic Estimation: Theory}

\subsection{Abstract}

A signal enhancement method is presented which recovers signal from noise by a judicious combination of a priori knowledge to define the desired feasible solutions, and a set theoretic estimation technique for restoring signal properties that have been lost due to noise contamination. The usefulness of this approach is illustrated by a simple NMR application using the matrix properties of Hankel structure and rank deficiency. The algorithm proposed by B. de Moor is adopted to solve the resulting best feasible approximation problem. It is shown via Monte Carlo simulations that a significant enhancement in spectral sensitivity and resolution can be achieved by applying this method as a preprocessing step prior to routine data analysis, at the expense of greater computational complexity. 


\subsection{Introduction}

The measured noisy free induction decays (FIDs) can usually be regarded as a sum of true signal and corrupt noise. NMR/MRI signal enhancement techniques may, therefore, be logically classified into three categories: (i) signal strength enhancement, (ii) noise strength reduction, and (iii) recovery of signal from noisy measurements. In this chapter, a mathematical signal enhancement method in category (iii) is presented, which seeks to reduce the degradation made on the excitation-response observations and to enhance the spectral/image estimators' performance. This object is achieved by using a priori knowledge to define the desired signal, and a set theoretic estimate to restore signal properties that have been lost due to noise corruption and measurement distortion. In the context of NMR/MRI experiments, a wide range of a priori knowledge arises from our thecretical and experimental understanding of the underlying spin system. This includes information on the spectra to be studied (e.g., number of peaks, lineshapes, coupling patterns, and relations between spectral parameters), attributes pertaining to the system that generates the FIDs (e.g., deterministic/stochastic information and autoregressive order), and probabilistic description of the external noise process (e.g., statistical mean, variance, and whiteness). Each piece of information can potentially be used to discriminate signal from noise and hence increase the precision of the signal recovery. The resulting estimate, which possesses all the known or hypothesized properties, should then more accurately portray the salient characteristics of the spin system and a signal enhancement can then 
be mathematically accomplished. This is the basic philosophical motivation for the approach of mathematical signal enhancement in the set theoretic framework.

\subsection{Best Feasible Approximation}

Set theoretic estimation is governed by the notion of feasibility and is constrained to produce solutions consistent with all available information $[1,2,3,4,5]$. Each piece of information is associated with a set in the solution space and the intersection of these sets represents the acceptable solutions. If $\left(\Psi_{i}\right)_{1 \leq i \leq I}$ is the collection of $I$ propositions representing a priori knowledge and $\Xi$ the solution space, a collection of so-called property sets $\left(S_{i}\right)_{1 \leq i \leq I}$ can be formally written as

$$
S_{i}=\left\{a \in \Xi \mid \Psi_{i} \text { holds for } a\right\}
$$

A set theoretic estimate is any object consistent with all available information, i.e., any point in the set intersection

$$
S=\bigcap_{i=1}^{I} S_{i}=\left\{a \in \Xi \mid \forall i \in\{1, \cdots, I\} \Psi_{i} \text { holds for } a\right\}
$$

This set $S$ is called the feasibility set and the problem of set theoretic estimation reduces to finding a point in $S$. A pictorial description is given in Fig. 1.1.

In order to use the set theoretic approach most efficiently, one must first select the 


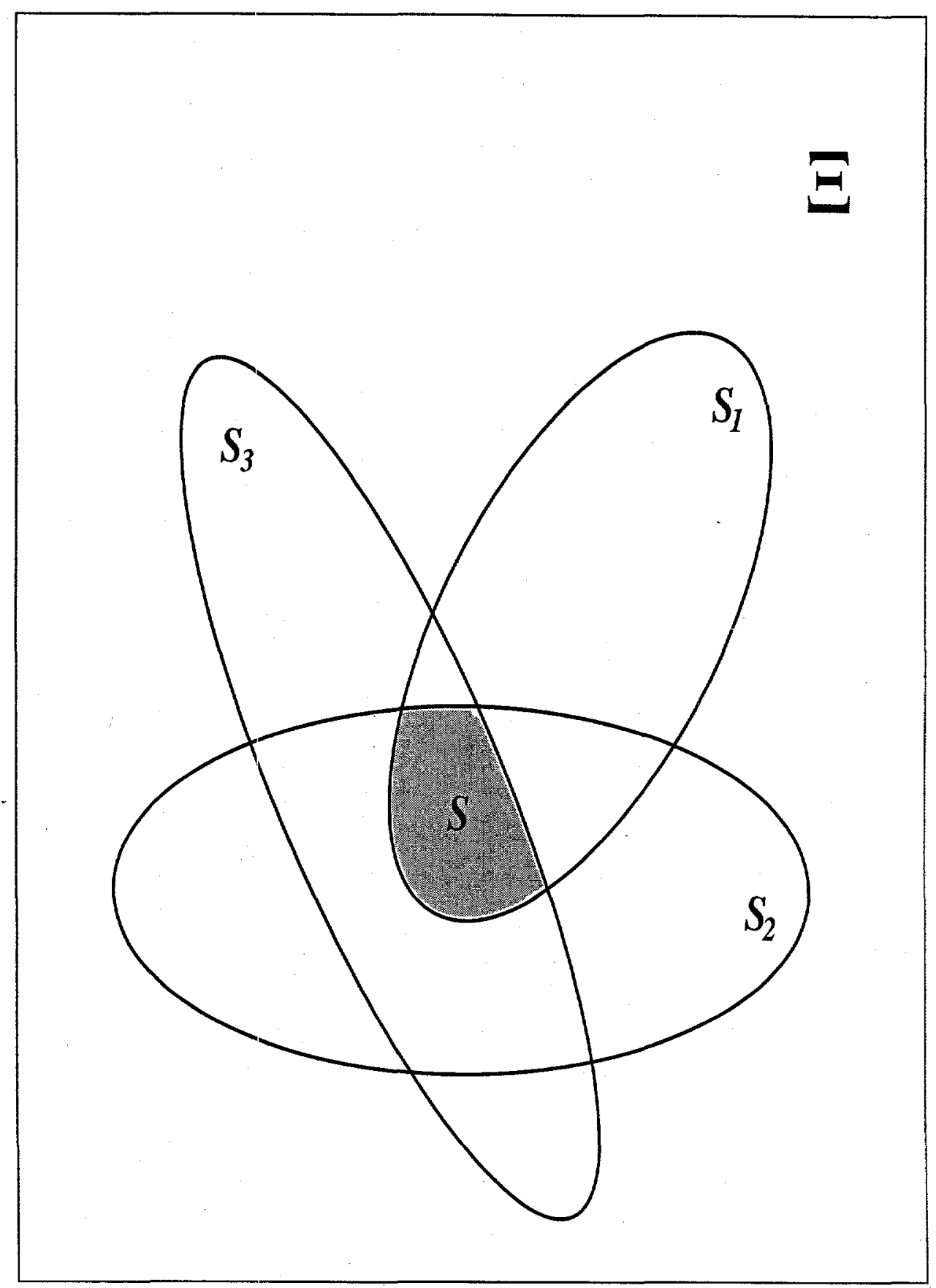

Figure 1.1: Set theoretic estimation. Feasibility set $S=S_{1} \cap S_{2} \cap S_{3}$. Any point in $S$ will be an equally acceptable solution in the set theoretic framework. 
solution space $\Xi$ which can describe all available information easily and accurately. A rule of thumb is to select a solution space that contains the objects directly described by most of the available information. The usual goal of NMR spectral estimation or MRI image processing is to obtain an estimate of the frequency or spatial response function of the underlying spin system from the measured FID, a time-series vector. Therefore, the space of complex vectors equipped with vector norms will be appropriate. For this purpose, we may temporarily assume the solution space $\Xi$ is a metric space denoted by $(\Gamma, d)$ in which $\Gamma$ is a set of vectors and $d(\boldsymbol{x}, \boldsymbol{y})$ is a metric that measures the distance between any two vectors $x, y$ of the set $\Gamma$. This distance measure $d(\boldsymbol{x}, \boldsymbol{y})$ is commonly referred to as the Euclidean norm of $(\boldsymbol{x}-\boldsymbol{y})$, i.e.,

$$
d(x, y)=\sqrt{\sum_{i}\left|x_{i}-y_{i}\right|^{2}}
$$

Specifically, $\left(S_{i}\right)_{1 \leq i \leq I}$ will then designate $I$ sets of vectors contained in $(\Gamma, d)$ that satisfy properties $\left(\Psi_{i}\right)_{1 \leq i \leq I}$, with $S$ being the intersection of these vector sets. Any point in $S$ will be an equally acceptable solution in the set theoretic framework.

When there is no noise contamination, the FID itself and its resulting data matrix, covariance matrix, and autocorrelation matrix are known to possess certain properties $[6,7,4,5]$. Inevitable noise in measurement, however, will result in a loss of some, or all, of these theoretical properties. If the noise perturbaition is reasonably small, a bound $\delta$ on the deviation of the noiseless FID from the empirically generated 
noisy FID $x$ may arise from the a priori constraint imposed by noise variance[8]. This constraint confines estimates to lie in a ball $B$ with center $\boldsymbol{x}$ and radius $\delta$, i.e., $B=\{\boldsymbol{y} \in \Xi \mid d(\boldsymbol{x}, \boldsymbol{y}) \leq \delta\}$, as shown in Fig. 1.2. Such a constraint can significantly reduce the set of feasible solutions and thereby improve the reliability of the set theoretic estimates. If $\delta$ can not be determined reliably, one can still exploit the constraint by choosing as a solution the feasible point $x^{*} \in S$ that lies nearest to $x$. Such a point, $\boldsymbol{x}^{*}$, will be guaranteed to lie in the intersection of $S$ and any ball $B$ centered at $\boldsymbol{x}$ and intersecting with $S$. Geometrically, $\boldsymbol{x}^{*}$ is simply the projection of $x$ onto $S$, i.e., $\boldsymbol{x}^{*}=\Pi(\boldsymbol{x})$ (see Fig. 1.2). Consequently, an enhanced signal can be formally obtained by finding the projection operator $\Pi$ or, equivalently, by solving the following best feasible approximation problem

$$
\inf _{\boldsymbol{y} \in S} d(\boldsymbol{x}, \boldsymbol{y})
$$

where "inf" denotes the greatest lower bound operator.

\subsection{Monte Carlo Simulations}

To illustrate the above abstract concepts, in the following we will consider a simple NMR example based on exponential FID modelling. The entire computation has been carried out on a SGI Indigo IRIS-4000 workstation.

If the spin relaxation can be treated as a first order process, the measured NMR 


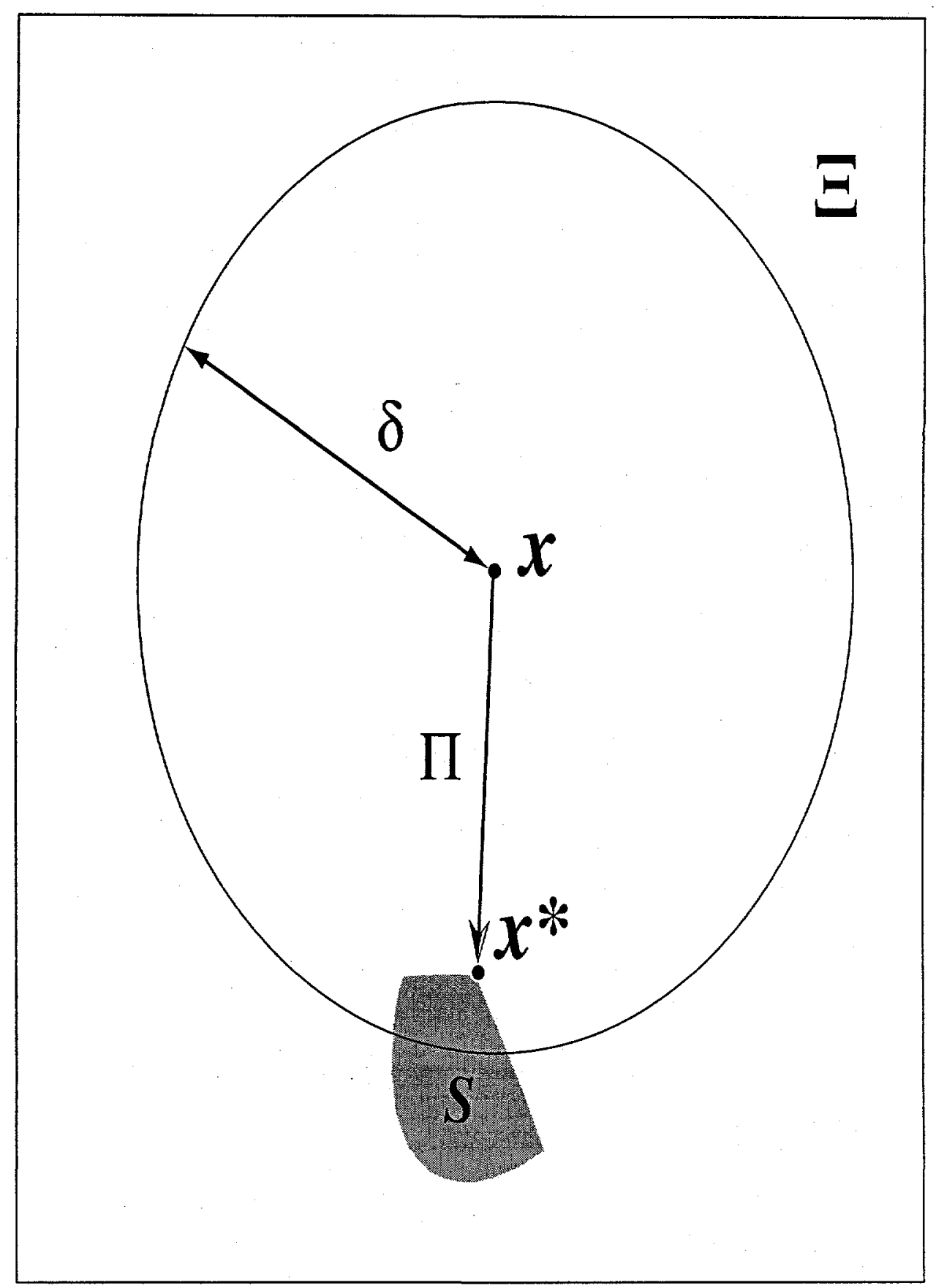

Figure 1.2: Best feasible approximation. $\boldsymbol{x}^{*}$, defined by the projection of $\boldsymbol{x}$ onto $S$, will be guaranteed to lie in the intersection of $S$ and any ball $B$ centered at $x$ and intersecting with $S$. 
impulse response will be a sum of exponentially damped sinusoids perturbed by additive noise,

$$
x_{n}=\sum_{i=1}^{M} a_{i} \exp \left(j \theta_{i}\right) \exp \left[\left(-\alpha_{i}+j 2 \pi f_{i}\right) n\right]+w_{n} \quad n=0,1, \ldots, N-1
$$

where $a_{i}, \alpha_{i}, f_{i}, \theta_{i}$ represent the amplitudes, damping factors (inverse time constants), frequencies, and phases respectively of the $M$ distinct exponentials; $w=$ $\left[w_{0}, w_{1}, \ldots, w_{N-1}\right]^{T}$ is the additive noise perturbation, $N$ is the number of data points, and $j$ is used to denote $\sqrt{-1}$. Consider the data matrix $\boldsymbol{X}$ formed from such a FID $\boldsymbol{x}=\left[x_{0}, x_{1}, \ldots, x_{N-1}\right]^{T}$

$$
\boldsymbol{X}(\boldsymbol{x})=\left[\begin{array}{cccc}
x_{0} & x_{1} & \cdots & x_{N-L} \\
x_{1} & x_{2} & \cdots & x_{N-L+1} \\
\vdots & \vdots & \ddots & \vdots \\
x_{L} & x_{L+1} & \cdots & x_{N-1}
\end{array}\right]
$$

where "T" de:zotes matrix transpose and $L$ is a parameter chosen by the user. It follows that $\boldsymbol{X}$, when constructed from noiseless FID, possesses two important properties, (i) $\Psi_{1}$, Hankel structure, and (ii) $\Psi_{2}$, rank deficiency. A Hankel matrix is one which is symmetrical about any cross-diagonal, while the rank deficiency of $\boldsymbol{X}$ results from $\boldsymbol{x}$ being the impulse response of a finite dimensional linear system of relatively low order. Indeed, since exponential signals satisfy recursive homogeneous difference 
equations[9], then if the FID $\boldsymbol{x}$ is the sum of $M$ noiseless complex exponentials with $M \leq \min (L+1, N-L+1), \boldsymbol{X}$ will be of rank $M$. Similarly, if $\boldsymbol{x}$ contains only the real or imaginary part of complex exponentials, $\boldsymbol{X}$ will be of rank $2 M$, since cosine or sine functions can always be decomposed into the sum of two complex exponentials with conjugate exponents.

With above a priori knowledge, the general expression of the best feasible approximation problem of Eq. (1.4) can be specified as

$$
\inf \boldsymbol{y} \in S=S_{1} \cap S_{2} d(\boldsymbol{x}, \boldsymbol{y}) \text {, }
$$

where property sets $S_{1}=\{\boldsymbol{x} \in \Xi \mid \boldsymbol{X}(\boldsymbol{x})$ is a Hankel matrix $\}, S_{2}=\{\boldsymbol{x} \in \Xi \mid \operatorname{rank}[\boldsymbol{X}(\boldsymbol{x})]=$ $M\}$, and $\boldsymbol{X}(\boldsymbol{x}) \in C^{(L+1) \times(N-L+1)}$. The specific projection operator required to solve Eq. (1.7) has recently been developed in the context of constrained total least squares $[15,16,17,4,18,19,20]$. The algorithm proposed by B. de Moor $[19,20]$ is adopted here, and its MATLAB implementation is listed in the Appendix. Unlike some other algorithms that only provide an approximate solution [17, 4, 18], B. de Moor's algorithm can offer the exact optimal solution to Eq. (1.7), as can be checked via the orthogonality condition $\left(x-x^{*}\right)^{T} x^{*}=0$ (which is necessary for optimality).

Within the last decade, much research activity has been focused on formulating and comparing alternative means of NMR spectral/parameter estimation[17, 19, 6, 7, 21, 22]. For example, least squares Prony method (LS-Prony) and its variants have 
been shown to be useful for NMR/MRI quantification $[17,19,6,7,21,22,23,24$, $25,26]$. LS-Prony embeds the nonlinear aspect of exponential model into a judicious combination of linear prediction and polynomial factoring. Its principles have been extensively documented[ 7,27$]$, but in summary, one first solves the following overdetermined linear prediction equations for the coefficients $\left\{b_{m}\right\}_{1 \leq m \leq q}$ of the prediction polynomial by minimizing the prediction error $\sum_{n=q}^{N-1}\left|x_{n}-\hat{x}_{n}\right|^{2}$,

$$
\left[\begin{array}{cccc}
x_{q-1} & x_{q-2} & \cdots & x_{0} \\
x_{q} & x_{q-1} & \cdots & x_{1} \\
\vdots & \vdots & \ddots & \vdots \\
x_{N-2} & x_{N-3} & \cdots & x_{N-q-1}
\end{array}\right]\left[\begin{array}{c}
b_{1} \\
b_{2} \\
\vdots \\
b_{q}
\end{array}\right] \approx\left[\begin{array}{c}
x_{q} \\
x_{q+1} \\
\vdots \\
x_{N-1}
\end{array}\right]
$$

where $\hat{x}_{n}=\sum_{m=1}^{q} b_{m} x_{n-m}$ is the predicted value of $x_{n}$. The roots of the prediction polynomial $P(z)=1-\sum_{m=1, q} b_{m} z^{-m}$ then produce the signal poles $z_{i}=\exp \left(-\alpha_{i}+\right.$ $\left.j 2 \pi f_{i}\right)$. Once damping factors $\alpha_{i}$ and frequencies $f_{i}$ are known, the amplitudes $a_{i}$ and phases $\theta_{i}$ can finally be solved by a general linear least-squares analysis.

It has been shown that LS-Prony and its variants can achieve a resolution in chemical shifts far exceeding that of conventional Fourier transform[7, 27]. However, their estimation accuracy and resolving ability rapidly deteriorate as the signal-to-noise ratio $(\mathrm{S} / \mathrm{N})$ drops to a critically low value[17, 19, 6, 7, 21, 22, 23, 24, 25, 26]. To reduce this problem, the enhancement procedure of Eq. (1.7) can be invoked to first "clean up" the FID before it is processed, for example; using LS-Prony. In the following, 
Monte Carlo simulations will be used to evaluate the resulting improvements in spectral sensitivity (Simulation I) and resolution (Simulation II). These examples have previously been used to compare the performances of various spectral estimators[28]. Spectral parameters are estimated by the standard LS-Prony subroutine listed in Ref. [7] using the same noise-free data set, but 400 different realizations of the noise for each considered $\mathrm{S} / \mathrm{N}$ with, and without, the enhancement preprocessing. The noise is assumed to be Gaussian and white with zero mean and variance $\sigma_{w}^{2}$. The signal-to-noise ratio $(\mathrm{S} / \mathrm{N})$ is here defined as

$$
\mathrm{S} / \mathrm{N} \equiv 10 \log _{10} \frac{\sum_{i} a_{i}^{2}}{\sigma_{w}^{2}}(\mathrm{~dB})
$$

Simulation I: Sensitivity Enhancement. The synthesized FIDs are given by $x_{n}=a \exp (-\alpha n) \cos (2 \pi f n+\theta)+w_{n}$ with damping factor $\alpha=0.05$, normalized frequency $f=0.2$, amplitude $a=1.0$, phase $\theta=0$, and $n=0, \ldots, 25$. Figure 1.3 (a) and (b) summarise the Cramér-Rao lower bounds and the statistics in damping factor $\alpha$ and frequency $f$,respectively. The Cramér-Rao lower bound predicts the best possible performance (smallest variance) for any unbiased estimator; its calculation has been outlined in several places[23, 29]. It is shown in Fig. 1.3 that the enhancement preprocessing effectively improves the estimate statistics as if the spectral sensitivity has been increased by $12 \mathrm{~dB}$ or, equivalently, the acquisition time has been reduced by a factor of 250 , as estimated from reduction in the mean square error of damping 
factors $\alpha$ or frequencies $f$ (mean square error is the sum of the variance and the square of bias). Over the range of noise levels investigated, the enhanced estimates are now essentially near-optimal in the sense that their biases are almost equal to zero and their variances are very close to their theoretical lower bounds.

Simulation II: Resolution Enhancement. The synthesized FIDs consist of two closely spaced resonances exceeding the resolving ability of FT:

$x_{n}=\sum_{i=1}^{2} a_{i} \exp \left(-\alpha_{i} n\right) \cos \left(2 \pi f_{i} n+\theta_{i}\right)+w_{n}$ with damping factors $\alpha_{1}=\alpha_{2}=0.05$, normalized frequencies $f_{1}=0.20, f_{2}=0.22$, amplitudes $a_{1}=a_{2}=1.0$, phases $\theta_{1}=\theta_{2}=0$, and $n=0, \ldots, 25$. The estimation is judged to have failed if the peaks are not both resolved within two standard deviations (given by the CramérRao lower bounds) of the exact normalized frequencies. Fig. 1.4 shows the success rate as a function of $\mathrm{S} / \mathrm{N}$. In this case, the enhancement preprocessing not only provides much higher estimation accuracy (similar to Simulation I, data not shown) but also has a "breakdown" threshold for resolution that is much lower by $\approx 19 \mathrm{~dB}$ (as estimated from the success rate).

\subsection{Conclusion}

There are many different types of problems which require signal enhancement for a solution. It is desirable, thorefore, to make effective use of the observed data by using some novel mathematical methods. Set theoretic estimation is a technique which 

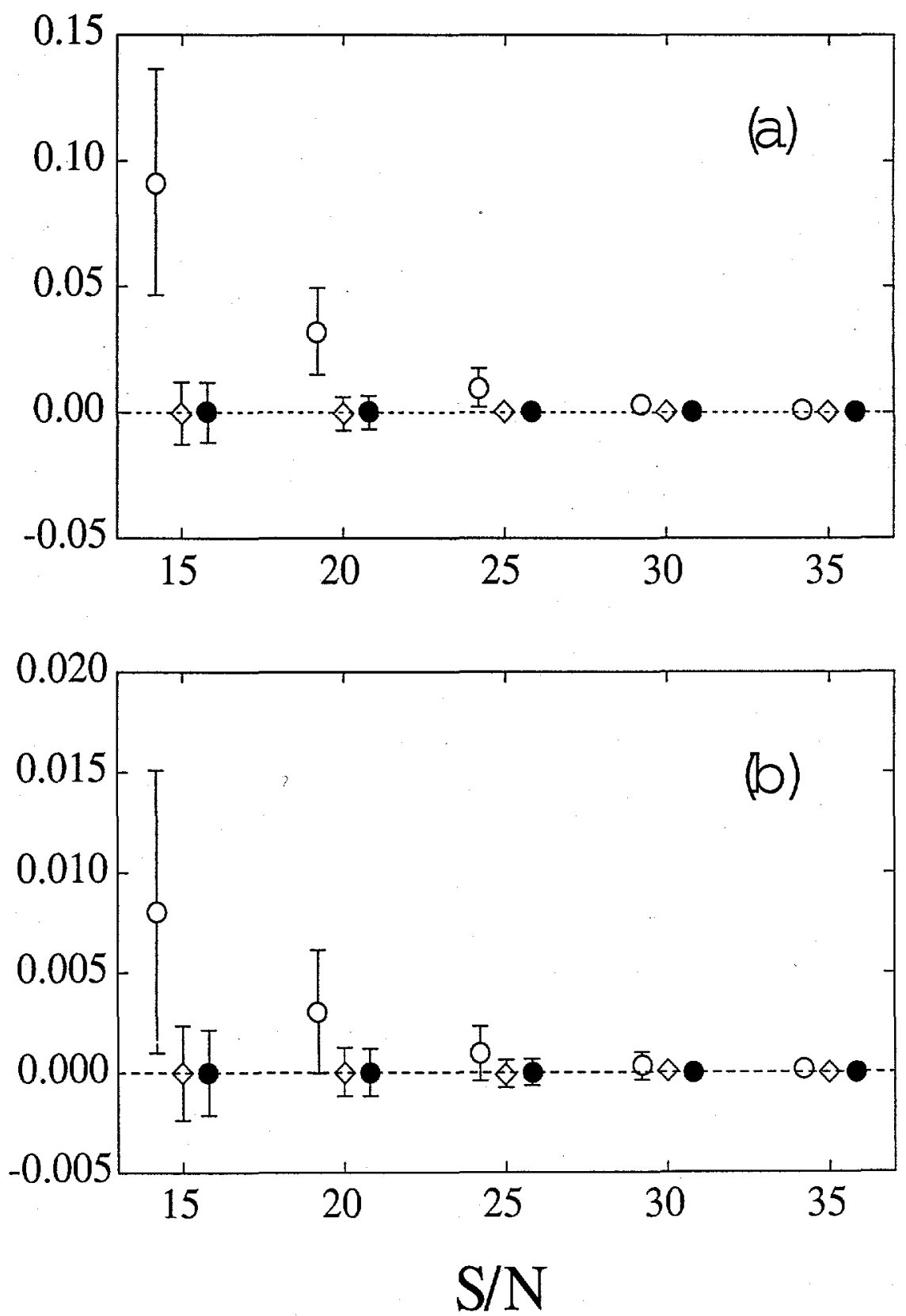

Figure 1.3: Estimated bias \pm std (standard deviation) in the (a) damping factor $\alpha$ and (b) frequency $f$ of Simulation I; (o) LS-Prony only, ( $\diamond)$ LS-Prony with enhancement preprocessing, and $(\bullet)$ Cramér-Rao lower bounds. 


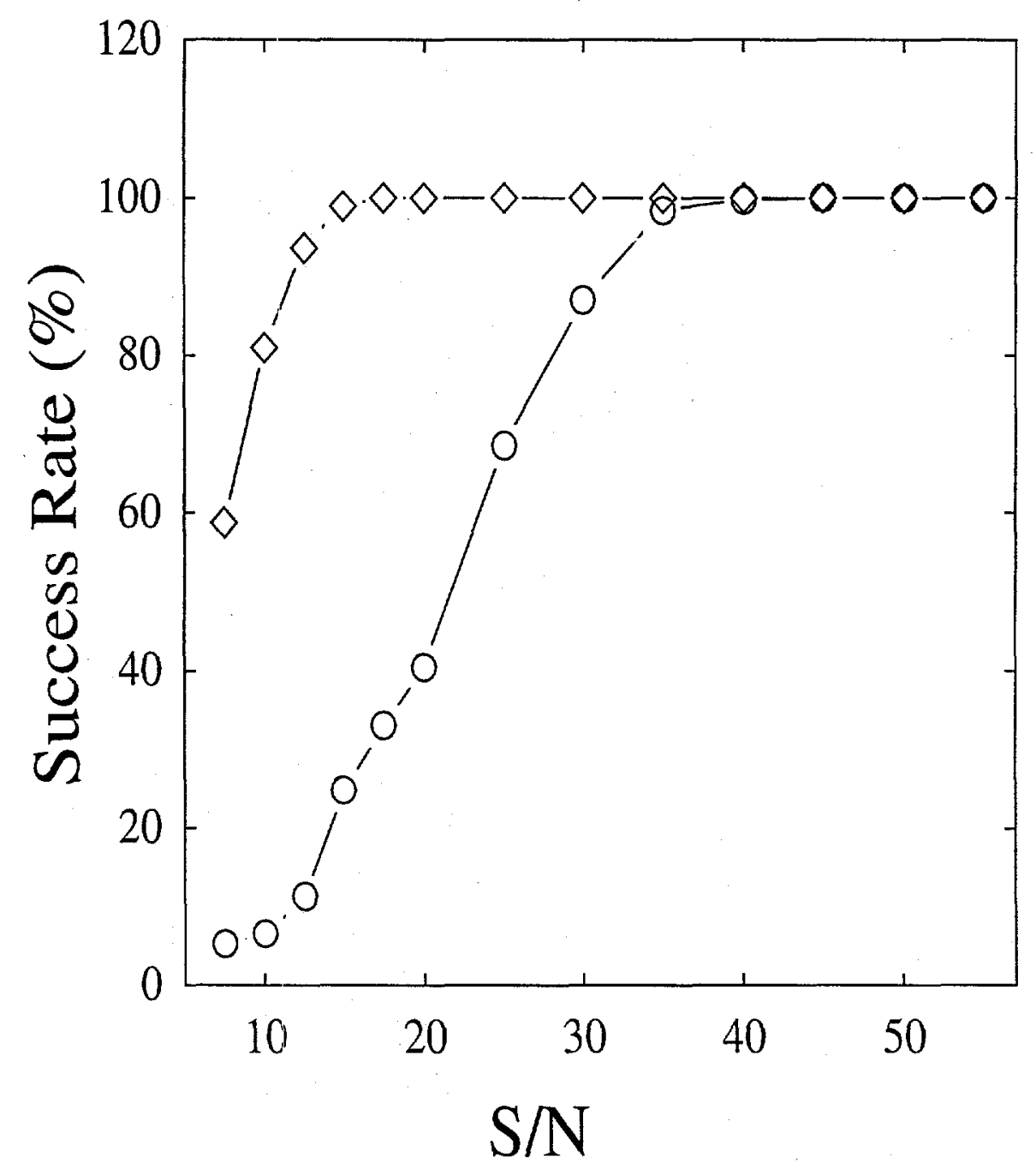

Figure 1.4: Success rate for resolving the closely spaced resonances of Simulation II;(o) LS-Prony only, ( $\diamond)$ LS-Prony with enhancement preprocessing. 
provides a set of solutions defined as the class of objects consistent with all information arising from a priori knowledge and the observed data set. If necessary, the unique feasible solution can be obtained from the feasibility set by optimizing some chosen objective function. This is exactly the case for NMR/MRI signal recovery where the true signal is known to lie near a reference or a prototype signal and the object function is simply the distance measurement. The resulting best feasible solution can be considered as a "clean" version of the original noisy data in which the effect of noise corruption has been significantly mitigated. This is illustrated by means of a rudimentary NMR application invoking the matrix properties of Hankel structure and rank deficiency. Monte Carlo simulations show that the signal enhancement preprocessing enables the LS-Prony method to produce effective estimates in a S/N environment $10-20 \mathrm{~dB}$ lower than would have been otherwise possible.

The concept of set theoretic estimation can be applied to a large number of applications. The development of better feasibility algorithms and incorporation of more signal and noise properties are the critical steps in broadening the scope of the applications of set theoretic estimation in NMR/MRI. The former เequests algorithmic procedures for obtaining an approximation of the optimal solution, while the latter usually entails some degree of ingenuity on the user's part to identify specific signal/noise properties and data representation that apply to a given problem. These issues shall be discussed in next chapter. 


\section{Bibliography}

[1] P. L. Combettes, Proc. IEEE 81, 182 (1993).

[2] J. R. Deller, IEEE ASSP Mag. 6, 4 (1989).

[3] H. J. Trussell and M. R. Civanlar, IEEE Trans. Acoust. Speech Signal Process. 32, 201 (1984).

[4] J. A. Cadzow, IEEE Trans. Acoust. Speech Signal Process. ASSP-36, 49 (1988).

[5] N. E. Hurt, Acta Applicandae Mathematicae 23, 145 (1991).

[6] S. M. Kay, "Modern Spectral Estimation," Prentice-Hall, Englewood Cliffs, New Jersey, 1987.

[7] S. L. Marple, "Digital Spectral Analysis with Applications," Prentice-Hall, Englewood Cliffs, New Jersey, 1987.

[8] P. L. Combettes, IEEE Trans. Image Process. 2, 269 (1993).

[9] A. V. Oppenheim and R. W. Schafer, "Digital Signal Processing," Prentice-Hall, Englewood Cliffs, New Jersey, 1975. 
[10] H. Park, J. B. Rosen, and S. van Huffel, in "SVD and Signal Processing III: Algorithms, Architectures, and Applications" (M. Moonen and B. De Moor, Eds.), pp. 399-406, Elsevier Science, New York, 1995.

[11] T. J. Abatzoglou, J. M. Mendel, and G. A. Harada, IEEE Trans. Signal Process. 39, 1070 (1991).

[12] J. H. McClellan and D. Lee, IEEE Trans. Signal Process. 39, 509 (1991).

[13] Y. Bresler and A. Macovski, IEEE Trans. Acoust. Speech Signal Process. ASSP34, 1081 (1986).

[14] B. de Moor, Linear Algebra and Its Applications 188-189, 163 (1993).

[15] B. de Moor, IEEE Trans. Signal Process. 42, 3104 (1994).

[16] R. K. Harris and B. E. Mann (Eds.), "NMR and the Periodic Table," Academic Press, London, 1978.

[17] R. de Beer and D. van Ormondt, in "NMR Basic Principles and Progress" (P. Diehl, E. Fluck, H. Gunther, R. Kosfeld, and J. Seelig, Eds.), Vol. 26, pp. 201, Springer-Verlag, Berlin/Heidelberg, 1992.

[18] J. J. Led and H. Gesmar, Chem. Rev. 91, 1413 (1991).

[19] R. E. Hoffman and G. C. Levy, Prog. NMR Spectrosc. 23, 211 (1991). 
[20] R. Roy, B. G. Sumpter, G. A. Pfeffer, S. K. Gray, and D. W. Noid, Physics Reports 205, 109 (1991).

[21] H. Gesmar, J. J. Led, and F. Abildgaard, Prog. NMR Spectrosc. 22, 255 (1990).

[22] D. S. Stephenson, Prog. NMR Spectrosc. 20, 515 (1988).

[23] R. Kumaresan and D. W. Tufts, IEEE Trans. Acoust. Speech Signal Process. ASSP-30, 833 (1982).

[24] H. Barkhuijsen, R. de Beer, W. M. M. J. Bovee, and D. van Ormondt, J. Magn. Reson. 61, 465 (1985).

[25] P. Barone, L. Guidoni, R. Ragona, V. Viti, E. Furman, and H. Degani, J. Magn. Reson. B 105, 137 (1994).

[26] P. Barone and G. Sebastiani, IEEE Trans. Med. Imaging 11, 250 (1992).

[27] L. L. Scharf, "Statistical Signal Processing: Detection, Estimation, and Time Series Analysis," Addison-Wesley, Massachusetts, 1991.

[28] T. Yu, IEEE Proc. F' 137, 192 (1990).

[29] H. Barkhuijsen, R. de Beer, D. van Ormondt, J. Magn. Reson. 67, 371 (1986).

[30] The Mathworks, Inc., "MATLAB Reference Guide," South Natick, Massachusetts, 1992. 


\section{Chapter 2}

\section{Set Theoretic Estimation:}

\section{Algorithm}

\subsection{Abstract}

The development of better feasibility algorithms and incorporation of more signal and noise properties are the critical steps in broadening the scope of the applications of set theoretic estimation in NMR/MRI. Among various novel algorithms, the feasibility algorithm of iterative parallel projections demonstrates certain theoretical and computational advantages for practical NMR applications. The effects of noise corruption, measurement distortion, and theoretical mismatch can be significantly mitigated through the process of iteratively and simultaneously modifying the noisecorrupted data set to the smallest degree necessary so that it possesses a collection 
of prescribed signal properties and also lies closest to the original data set. The usefulness of this approach is demonstrated by experimental ${ }^{43} \mathrm{Ca}$ and ${ }^{25} \mathrm{Mg}$ NMR spectra.

\subsection{Introduction}

For the reader's convenience, the concept of set theoretic estimation is outlined below. If $\left(\Psi_{i}\right)_{1 \leq i \leq I}$ is the collection of $I$ propositions representing a priori knowledge and $\Xi$ the solution space, a collection of so-called property sets $\left(S_{i}\right)_{1 \leq i \leq I}$ can be constructed in a propositional manner, namely,

$$
S_{i}=\left\{a \in \Xi \mid \Psi_{i} \text { holds for } a\right\} \text {. }
$$

A set theoretic estimate is any object consistent with all available information, i.e., any point in the set intersection

$$
S=\bigcap_{i=1}^{I} S_{i}=\left\{a \in \Xi \mid \forall i \in\{1, \cdots, I\} \Psi_{i} \text { holds for } a\right\}
$$

A signal-enhanced data set (constructed from the FID vector), $\boldsymbol{Y}$, can be formally obtained by solving the following best feasible approximation problem

$$
\inf _{Y \in S} d(\boldsymbol{X}, \boldsymbol{Y})
$$


where "inf" denotes the greatest lower bound operator and $\boldsymbol{X}$ is the measured noisy data set. This problem is of significance in many fields of research, and a number of algorithms have been proposed in the applied mathematics literature $[6,7,8,9,10,11]$. The selection of an algorithm mainly depends on the metric and geometric attributes of the property sets $\left(S_{k}\right)_{1 \leq k \leq M}$ and the structure of the underlying solution space $\Xi$. Because Eq. (2.3) can not usually be solved in one step, most feasibility algorithms are recursive and consist of building a sequence that converges to a point in the feasibility set.

\subsection{Iterative Parallel Projections}

The feasibility algorithm of iterative parallel projections $[1,16,17,18]$ in the framework of set theoretic estimation constitutes a particularly effective means to solve the best feasible approximation problem of Eq. (2.3). Let us first assume the exact solution is obtained by the projection operator $\Pi$, i.e., $\boldsymbol{Y}=\Pi(\boldsymbol{X})$. Similarly, the projection operator onto $S_{k}$ is denoted by $\Pi_{S_{k}}$. When two or more of the relatively simple property sets $\left(S_{k}\right)_{1 \leq k \leq M}$ are employed to form the feasibility set $S$, the associated projection operator $\Pi$ generally does not have an analytical form. This is due to the requirement that the solution must simultaneously lie in each of the property sets $\left(S_{k}\right)_{1 \leq k \leq M}$ and this set intersection can be extremely complicated (e.g., it is often nonconvex). In such cases, it is necessary to resort to algorithmic procedures for obtaining an approximation of the optimal solution. 
The algorithm of iterative parallel projections, instead of attacking Eq. (2.3) directly, exploits the techniques of best approximation and nonlinear programming [19] to solve the potentially more tractable set of problems

$$
\inf _{\boldsymbol{Y}_{\in S_{k}}} d(\boldsymbol{X}, \boldsymbol{Y}), 1 \leq k \leq M
$$

It then invokes the technique of parallel projections to constitute a local approximate solution to Eq. (2.3), which consists of the following recursive sequence

$$
\boldsymbol{X}^{(j)}=\Pi\left(\boldsymbol{X}^{(j-1)}\right)=\sum_{k=1}^{M} \omega_{k} \Pi_{S_{k}}\left(\boldsymbol{X}^{(j-1)}\right)
$$

where $j \geq 1, X^{(0)}$ is the initial estimate, and the weight satisfies $\sum_{k=1}^{M} \omega_{k}=1$. In such a scheme, all the property sets are activated simultaneously and the new iteration is a combination of the projections of the current iterate onto each property set. This algorithm has been shown to converge to a feasible point locally, as long as $\boldsymbol{X}^{(0)}$ lies in the region of attraction $[1,16,17,18]$.

In the framework of set theoretic estimation, most algorithms require that $\left(S_{k}\right)_{1 \leq k \leq M}$ be closed and convex setsi, or be describable by linear projection operators. Unfortunately, such restrictions are often too severe for matrix properties of interest as exemplified later by the singular value decomposition (SVD) characterization of matrix rank property. The main feature of the iterative parallel projections, therefore, lies in its ability to loosen these restrictions and only require that $\left(S_{k}\right)_{1 \leq k \leq M}$ be 
closed. As is shortly shown, the possibility to use nonconvex sets is critical in many important applications. Another salient feature of iterative parallel projections is its parallelism: at every iteration the projections can be computed simultaneously and independently on a parallel computing architecture to considerably reduce the computational burden. From our numerical experience, it is also found that Eq. (2.5) generally converges in a very efficient manner.

\subsection{Sensitivity Enhancement in ${ }^{43} \mathrm{Ca}$ NMR}

Both magnesium and calcium occur widely in biological as well as in non-biological systems. The relation of these elements to biological structure and function is a subject of intense research [20]. Since these ions, because of their closed electron shells, lack other useful spectroscopic properties such as ESR, UV/Visible, or luminescence properties, progress in these studies has been mainly relied on NMR spectroscopy $[21,22,23,24]$.

The receptivity at natural isotopic abundance of ${ }^{43} \mathrm{Ca}$ is one of the lowest in the Periodic Table [25]. The problem of inherently small NMR signal can be greatly improved through the use of isotope enriched samples, however, at a prohibitive cost. Many important and potential studies, therefore, remain unexplored.

Let's now consider the data matrix $\boldsymbol{X}$ formed from the measured FID $x(1), x(2), \cdots$, 
$x(N)$,

$$
\boldsymbol{X}=\left[\begin{array}{cccc}
x(L+1) & x(L) & \cdots & x(1) \\
x(L+2) & x(L+1) & \cdots & x(2) \\
\vdots & \vdots & \ddots & \vdots \\
x(N) & x(N-1) & \cdots & x(N-L)
\end{array}\right]
$$

where $L$ is a parameter chosen by the user. Since the usual NMR spin relaxations in liquids are in the Markovian limit, the magnetizations can be characterized by exponentially damped relaxation. It follows that this data matrix $X$, when constructed from noiseless FID, possesses three important matrix properties $[6,11,25]$.

Property 1: Toeplitz siructure. The Toeplitz matrix has the property that all the elements along the diagonal are identical and so are those along each subdiagonal. Assume the unique Toeplitz matrix $\boldsymbol{X}_{T}$ that lies closest to an arbitrary matrix $\boldsymbol{X} \in$ $C^{m \times n}$ in the Frobenius norm sense is obtained by the projection $\Pi_{S_{T}}(X)$. It is found that the $k$ th subdiagonal elements of the resulting $\boldsymbol{X}_{T}$ equal the average value of the $k$ th subdiagonal elements of $\boldsymbol{X}$. Here $C^{m \times n}$ denotes the metric space consisting of all $m \times n$ complex valued matrices.

Property 2: Matrix rank. If the FID $x(1), x(2), \cdots, x(N)$ comprises a sum of $Q$ noiseless complex exponentials with $Q \leq \min (N-L, L)$, it follows that the corresponding noiseless data matrix $X$ possesses rank $Q$. Its proof is dependent on the fact that exponential signals satisfy homogeneous difference equations. The widely 
employed SVD provides a particularly useful tool for characterizing the matrix-rank property. Recall that the SVD of an arbitrary matrix $X \in C^{m \times n}$ is given by

$$
\boldsymbol{X}=\sum_{i=1}^{r} \sigma_{i} \boldsymbol{u}_{i} \boldsymbol{v}_{i}^{\dagger}=\sigma_{1} \boldsymbol{u}_{1} \boldsymbol{v}_{1}^{\dagger}+\sigma_{2} \boldsymbol{u}_{2} \boldsymbol{v}_{2}^{\dagger}+\cdots+\sigma_{r} \boldsymbol{u}_{r} \boldsymbol{v}_{r}^{\dagger}
$$

where "†" stands for taking the Hermitian conjugate, $r \leq \min (m, n)$ is equal to the rank of $\boldsymbol{X}$, the $\left(\sigma_{i}\right)_{1 \leq i \leq r}$ are real and nonnegative singular values ordered in the monotonically nonincreasing fashion $\sigma_{i} \geq \sigma_{i+1}$, and the $\left(u_{i}\right)_{1 \leq i \leq r}$ and $\left(v_{i}\right)_{1 \leq i \leq r}$ are the corresponding orthonormal left and right singular vectors, respectively $[4,5]$. The matrix $\boldsymbol{X}$ is thus constructed from the contribution of $r$ rank-one matrices weighted by the respective singular values. If $\sigma_{Q} \neq \sigma_{Q+1}$, the unique matrix $\boldsymbol{X}_{R}$ of $\operatorname{rank} Q$ or less contained in property set $\dot{S}_{R}$ that lies closest to $\boldsymbol{X}$ in the minimum Frobenius norm sense is given by the matrix-rank projection (Eckard-Young theorem [27])

$$
X_{R}=\Pi_{S_{R}}(X)=\sum_{i=1}^{Q} \sigma_{i} u_{i} v_{i}^{\dagger}
$$

The nonconvexity of $S_{R}$ is established by noting that the sum of two rank- $Q$ matrices can have a rank greater than $Q$.

Property 3: Subsequences. Assume $\boldsymbol{x}=\{x(1), x(2), \cdots, x(N)\}$ comprises a sum of $Q$ noiseless complex exponentials. This sequençe may be decimated by a positive 
integer $p \geq 2$ to form $p$ subsequences

$$
\boldsymbol{x}_{p: q}=\{x(q), x(p+q), \cdots, x(\operatorname{FIX}(N / p-1) p+q)\} .
$$

Here $1 \leq q \leq p$ and "FIX" denotes rounding toward zero. Each such subsequence will also comprise a sum of $Q$ complex exponentials with the exponents being equal to those of the original raised to the $p^{\text {th }}$ power. We can then construct the composite subsequence data matrix $\boldsymbol{X}_{p}$

$$
\boldsymbol{X}_{p}=\left[\begin{array}{c}
\boldsymbol{X}_{p: 1} \\
\boldsymbol{X}_{p: 2} \\
\vdots \\
\boldsymbol{X}_{p: p}
\end{array}\right]
$$

where submatrix $\boldsymbol{X}_{p: q}(1 \leq q \leq p)$ denotes the data matrix formed from subsequence $\boldsymbol{x}_{p: q}$ according to Eq. (2.6). It follows that $\boldsymbol{X}_{p}$ will also possess rank $Q$ [28]. The projector $\Pi_{S_{U}}$, associated with the subsequence property set $S_{U, p}$ for an arbitrary standard data matrix $X \in C^{m \times n}$ can be described as follows. (i) Extract the sequence $\boldsymbol{x}$ from $\boldsymbol{X}$. Decimate $\boldsymbol{x}$ by $p$ to form $p$ subsequences $\left(\boldsymbol{x}_{p: q}\right)_{1 \leq q \leq p}$. (ii) For each $\boldsymbol{x}_{p: q}$, form submatrix $\boldsymbol{X}_{p: q}$ following Eq. (2.6), and then construct the composite subsequence data matrix $\boldsymbol{X}_{p}$ by Eq. (2.10). (iii) Find the nearest rank $Q$ approximation to $\boldsymbol{X}_{p}$. (iv) Perform the inverse operation of (i) and (ii), i.e., extract the 
submatrix and subsequence and then form the enhanced standard data matrix $\boldsymbol{X}_{U, p}$. Consequently, $\boldsymbol{X}_{U, p}=\Pi_{S_{U, p}}(\boldsymbol{X})$.

With such knowledge, the signal enhancement method can be easily formulated as

$$
\boldsymbol{X}^{(j)}=\omega_{S_{T}} \Pi_{S_{T}}\left(\boldsymbol{X}^{(j-1)}\right)+\omega_{S_{R}} \Pi_{S_{R}}\left(\boldsymbol{X}^{(j-1)}\right)+\sum_{p=2}^{p_{\max }} \omega_{S_{U, p}} \Pi_{S_{U, p}}\left(\boldsymbol{X}^{(j-1)}\right)
$$

with $\boldsymbol{X}^{(0)}$ the original noisy data matrix. The iterations can be stopped when negligible improvement in the projection is observed, which leads to the stopping rule

$$
\frac{d\left(\boldsymbol{X}^{(j)}, \boldsymbol{X}^{(j-1)}\right)}{d\left(\boldsymbol{X}^{(0)}, \mathbf{0}\right)} \leq \epsilon
$$

where 0 is the null matrix and $\epsilon$ is a suitably small positive number. Once the iterative procedure converges to a specific matrix, we can extract the spectral parameters by various quantification methods or just recover the signal-enhanced FID [refer to Eq. (2.6)] and then obtain the conventional FT spectrum.

We now apply Eq. (2.11) to NMR studies on $\mathrm{Ca}^{2+}$ complexation by using natural abundance ${ }^{43} \mathrm{Ca}$ samples. Figure 2.1a presents the original FT spectrum with 10,000 scans obtained at $300 \mathrm{~K}$ on a Bruker MSL-400 spectrometer for aqueous solution containing $0.20 \mathrm{M} \mathrm{CaCl}_{2}$ and $0.10 \mathrm{M}$ sodium EDTA. From the chemical composition of the solution, it is known that the frequency spectrum should show two Lorentzian peaks corresponding to free $\mathrm{Ca}^{2+}$ ion and Ca-EDTA complex. Unfortunately, these spectral 
features are now buried in a high level background noise. We have also performed another measurement under the same experimental conditions except for an increase of number of scans from 10,000 to 1,700,000. Its FT spectrum is depicted in Fig. 2.1d, which will be used as the "true" spectrum for comparison. The most commonly used technique to improve the signal-to-noise ratio $(\mathrm{S} / \mathrm{N})$ would be exponential multiplication, i.e., multiplying the noisy FID by an exponential decay function. Therefore, to compare the performance of our signal enhancement method with exponential multiplication, these two sensitivity enhanced versions of Fig. 2.1a are shown together in Fig. 2.1b (by exponential multiplication) and 2.1c (by Eq. (2.11)), respectively. The two peaks correspond to the $\mathrm{Ca}^{2+}$-EDTA (left) and free $\mathrm{Ca}^{2+}$ (right) states. The spectroscopy parameters used are: sampling interval $150 \mu \mathrm{s}$, deadtime delay $30 \mu \mathrm{s}$, pulse interval $400 \mathrm{~ms}$, and left shift 4 complex data points. Please notice the scale change in (b).

During the signal enhancement preprocessing, ten property sets are simultaneously activated. These include Toeplitz-structure property set $S_{T}$, matrix-rank property set $S_{R}$, and subsequence property sets $\left(S_{U, p}\right)_{2 \leq p \leq 9}$. Other algorithm parameters used are $N=256, L=192$ [Eq. (2.6)], $Q=2$ [Eq. (2.8)], $p_{\max }=9$ [Eq. (2.11)], and $\epsilon=0.004$ [Eq. (2.12)]. The algorithm convergence is shown in Fig. 2.2. Satisfactory results can in general be achieved within 10 iterations. Since no particular property set should be privileged in this case, the weights are taken to be equal, i.e., $\omega_{i}=1 / 10$. For problems in which some property sets are judged to be more critical than others, 
they should be assigned larger weights.

By comparing Fig. 2.1b with 2.1d, it is observed that the incorporation of exponential multiplication attenuates the noise contribution to some extent, however, at the expense of resolution degradation and intensity distortion. This problem arises because the generally used apodization technique is nothing but a linear operation, so that it treats noise in the same way as signal. Consequently, these spectral obstacles can not be surmounted without sacrificing something valuable, be it spectral resolution or sensitivity. This kind of artifact, however, does not occur in our signal enhancement method. In Fig. 2.1c, the signal-enhanced spectrum exhibits a very close approximation to the "true" spectrum (Fig. 2.1d). Therefore, this signal enhancement preprocessing not only effectively strips away noise that contaminates the original data, but also faithfully reconstructs the spectral details even with as few as 10,000 scans. This fact means that a factor of 170 reduction in NMR machine time might be obtained and, hence, the ${ }^{43} \mathrm{Ca}$ NMR studies without isotope-enriched samples might be possible. The price for such accomplishment is only 5 minutes CPU time in our SGI IRIS-4000 workstation.

\subsection{Resolution Enhancement in ${ }^{25} \mathrm{Mg}$ NMR}

Recently, NMR spectroscopy of enriched ${ }^{25} \mathrm{Mg}$ has been adapted as a useful tool in the studies of $\mathrm{Mg}^{2+}$ binding with biomolecules in solution [20, 21]. However, progress in these studies has been much retarded primarily because of experimental 

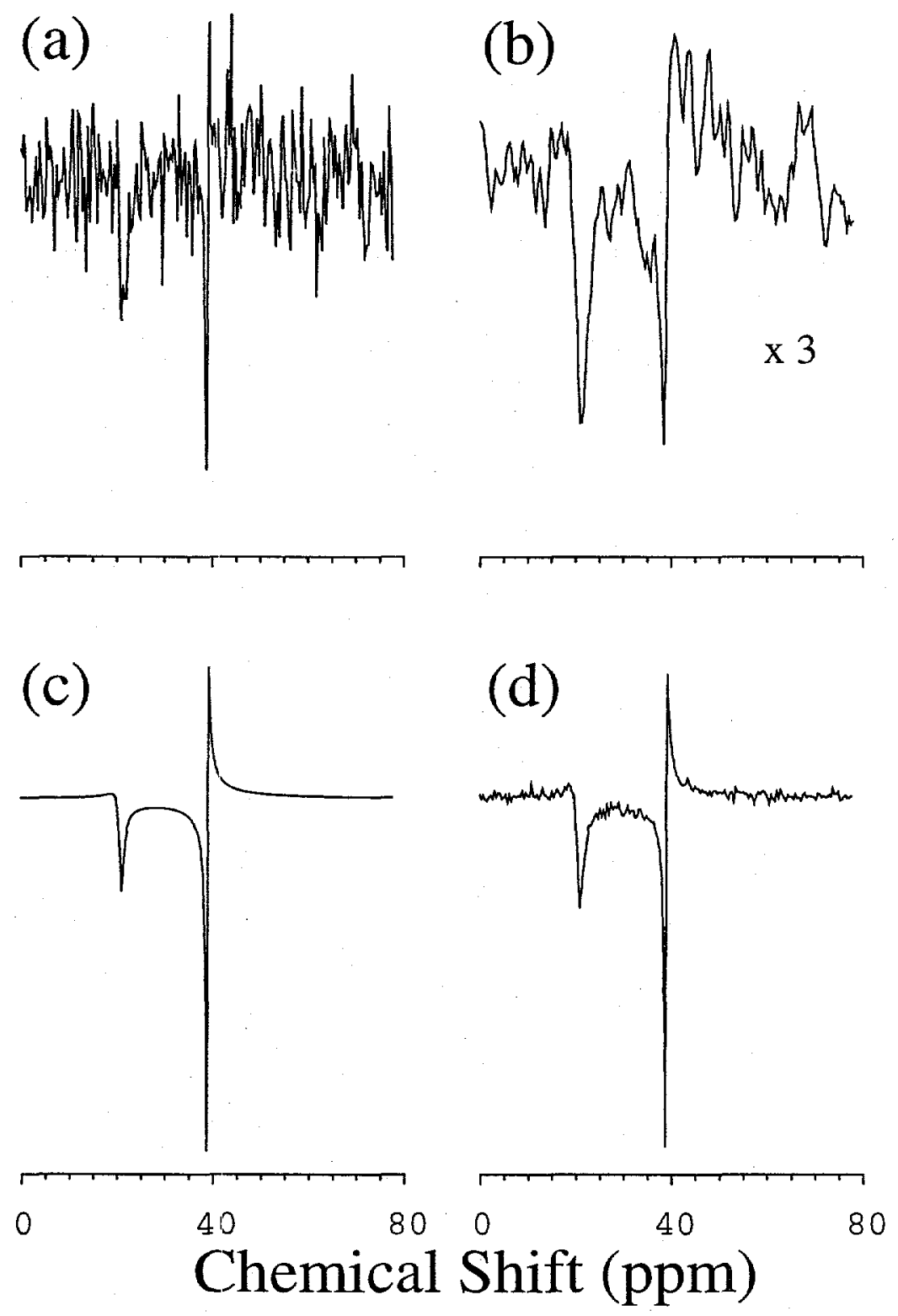

Figure 2.1: The natural abundance ${ }^{43} \mathrm{Ca}$ frequency spectra (real part without phase correction) obtained at $300 \mathrm{~K}$ on a $400 \mathrm{MHz}$ Bruker spectrometer for an aqueous solution containing $0.20 \mathrm{M} \mathrm{CaCl}_{2}$ and $0.10 \mathrm{M}$ sodium EDTA. (a) The direct FT of the FID with 10,000 scans; (b) Signal-enhanced spectrum of (a) obtained by multiplying the original measured FID by an exponential decay function, i.e., $x(n):=x(n) \mathrm{e}^{-\beta n}$ with $n=0 \cdots 255$ and $\beta=0.03$; (c) Signal-enhanced spectrum of (a) obtained by using signal enhancement preprocessing of Eq. (2.11); (d) The direct FT of the FID with $1,700,000$ scans, used as the "true" spectrum for comparison. 


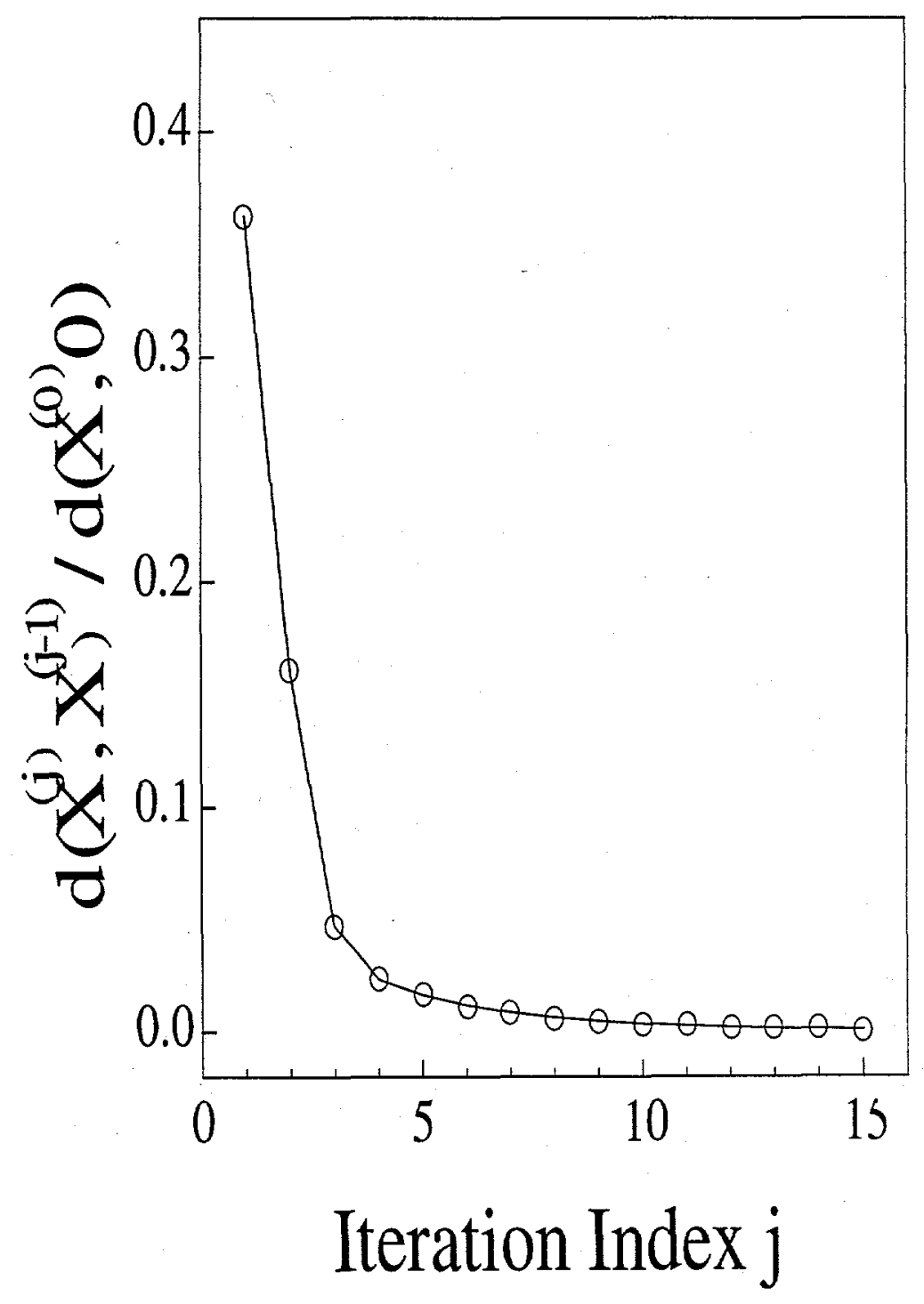

Figure 2.2: The algorithm convergence of the iterative parallel projections. 
inaccessibility due to poor chemical shift separation blurred further by low sensitivity, acoustic ringing, and sizable quadrupole relaxation effects $[22,23,24]$.

Within the last decade, there has been a flurry of research activity into formulating and comparing alternative means of NMR spectral estimation. The primary motivation has apparently been the development of techniques for enhanced spectral resolution. Among them, linear prediction (LP) has attracted considerable interest as an alternative approach to FT for quantification of NMR FIDs $[12,13,14,15,29,30,31,32]$. The success of $\mathrm{LP}$ resides in its predictive power to extend truncated FID for resolution enhancement, or to recalculate the initial data points from the remainder of the FID for baseline and phase corrections. It has been shown that LP as well as most of the other proposed estimators can achieve a resolution in chemical shifts far exceeding that of conventional FT. However, their resolving ability rapidly deteriorates as the signal-to-noise ratio $(\mathrm{S} / \mathrm{N})$ drops to a critical value.

To alleviate this problem, the signal enhancement method of Eq. (2.11) can be invoked to first "clean up" the FID being processed, and then a high-resolution spectral estimator can be applied to the signal-enhanced FID to extract the spectral parameters. Here, the LP with the Kumaresan-Tufts algorithm, LPSVD [29, 30], is selected to serve as the high-resolution spectral estimator due to its growing popularity in the NMR community. The result of LPSVD analysis is a table of spectral parameters (damping factor, frequency, amplitude, and phase) which either can be used to con- 
struct a frequency/time domain spectrum or can be used directly without the need for complicated peak searching, curve fitting, and intensity integration. It is well known that the ${ }^{25} \mathrm{Mg}$ chemical shift is very insensitive to the formation of complexes [24]. Therefore, we can use the example of ${ }^{25} \mathrm{Mg}$ binding with EDTA to verify the usefulness of this two-step procedure in resolving very closely spaced exponentially damped sinusoids under low $\mathrm{S} / \mathrm{N}$ environments. The FT spectra shown in Fig. 2.3a (500 scans) and 2.3c (30,000 scans) are obtained from an aqueous solution containing $0.80 \mathrm{M} \mathrm{MgCl}_{2}$ (natural abundance) and $0.60 \mathrm{M}$ sodium EDTA. To emphasize the fine spectral structure, only about $1 / 5$ of the full spectral width is shown here. The stock solution of $\mathrm{MgCl}_{2}$ was first standardized by titration with EDTA using Eriochrome Black $\mathrm{T}$ indicator. Under the experimental conditions, the $\mathrm{Mg}^{2+}$ ion is in the limit of slow exchange between complexed and uncomplexed sites and forms a 1:1 complex with EDTA. The signal-enhanced spectra (dash line) shown in Fig. 2.3b and 2.3d are obtained respectively from Fig. $2.3 \mathrm{a}$ and $2.3 \mathrm{c}$ by the signal enhancement algorithm of Eq. (2.11), while the two resolved components (solid line) are generated from further LPSVD analysis of the signal-enhanced FIDs. The algorithm parameters are the same as those used in the previous ${ }^{43} \mathrm{Ca}$ example. The two resolved components represent the $\mathrm{Mg}^{2+}-$ EDTA (right) and free $\mathrm{Mg}^{2+}$ states (left). After correcting the effect of deadtime and extrapolating to initial amplitudes, the $\left[\mathrm{Mg}^{2+}\right] /\left[\mathrm{Mg}^{2+}-\mathrm{EDTA}\right]$ ratios are estimated to be 0.32 (Fig. 2.3b) and 0.34 (Fig. 2.3d), which agree excellently with the stoichiometric ratio 0.33. By comparing the signal-enhanced spectrum (Fig. 2.3b) 
with the "true" spectrum (Fig. 2.3d), it might be concluded that the combined use of set theoretic estimation and high-resolution spectral estimator holds great promise in reducing the total accluisition time without significant loss of resolution. This is critical to, say, multi-dimensional in vivo NMR, where very poor sensitivity and resolution may arise from the low concentration of the sample species and limited data points in the higher dimensions. On the other hand, this example also reveals the inherent drawbacks of FT: limited resolution and its inability to distinguish between signal and noise. Consequently, in the absence of detailed knowledge about the spectral contents, FT should be used conservatively and carefully, particularly when overlapping peaks are to be accurately quantified or interpreted.

One point remaining to be addressed is the determination of the effective rank $Q$ in matrix-rank projection $\Pi_{S_{R}}$ and subsequerice projection $\Pi_{S_{U, p}}$. This can usually be done based on one's a priori knowledge about the number of exponential components in the FID or by examining the relative magnitudes of the singular values of the associated data matrix. It is found that the data matrix is generally almost rank deficient and there is a jump $\sigma_{Q} \gg \sigma_{Q+1}$ in its singular values, as exemplified in Fig. 2.4 for the data matrix constructed from the FID of Mg-EDTA shown in Fig. 2.3c. This distinction, however, will eventually become blurred when the spacing between resonance frequencies, the number of data points, and the $\mathrm{S} / \mathrm{N}$ all decrease. As discussed in Chapter 3, in such cases one can still resort to various statistical criteria based on the theories of perturbations of singular values and significance test [33]. It 


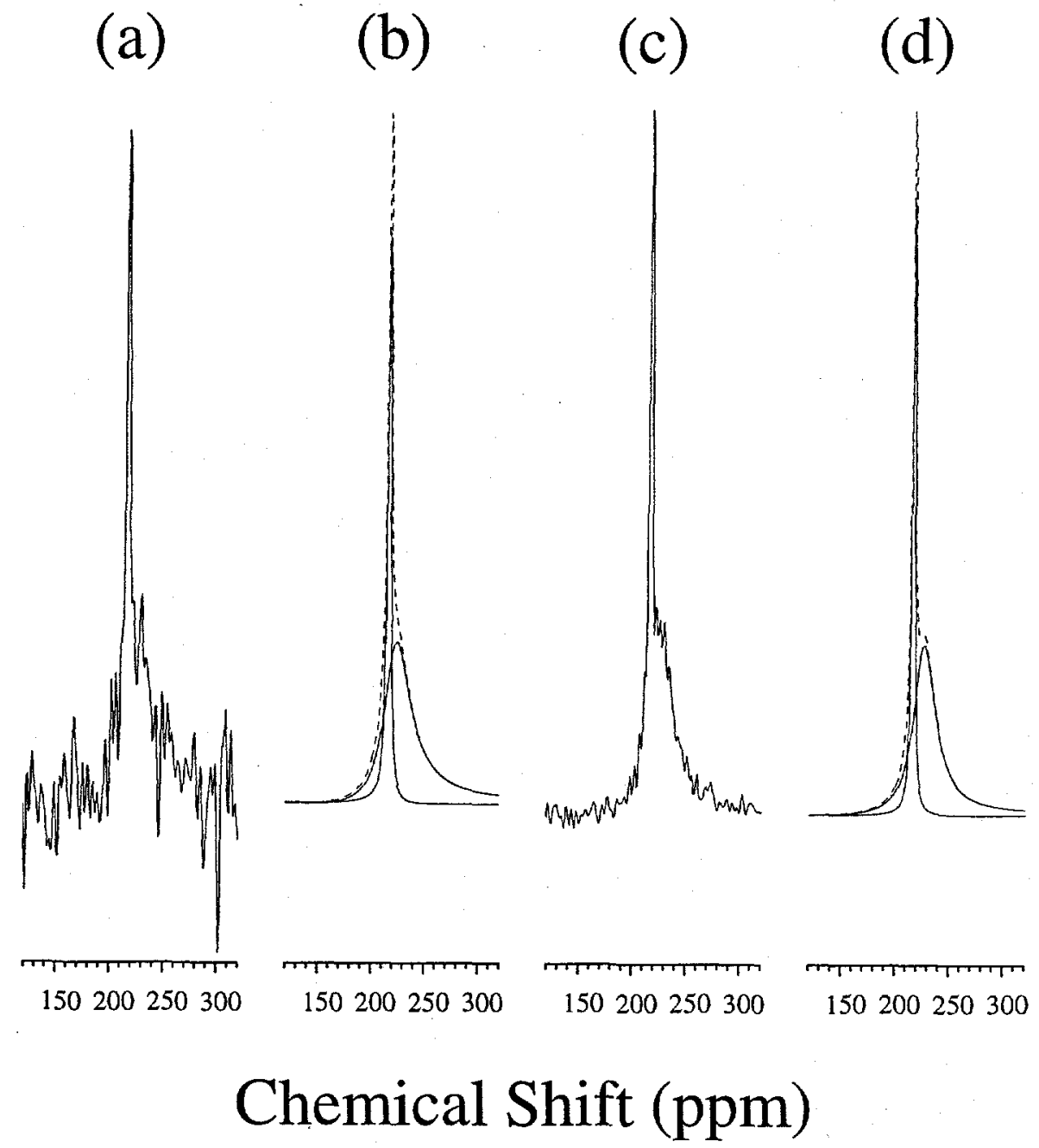

Figure 2.3: The natural abundance ${ }^{25} \mathrm{Mg}$ frequency spectra (real part with zeroth order phase correction) obtained at $300 \mathrm{~K}$ on a $400 \mathrm{MHz}$ Bruker spectrometer for an aqueous solution containing $0.80 \mathrm{M} \mathrm{MgCl}_{2}$ and $0.60 \mathrm{M}$ sodium EDTA. (a) The direct FT of the FID with 500 scans and 1K zero-filling; (b) Signal-enhanced spectrum of (a) obtained by the signal enhancement preprocessing (dash line) and then LPSVD analysis (solid line); (c) The direct FT of the FID with 30,000 scans, used as the "true" spectrum for comparison; (d) Signal-enhanced spectrum of (c) obtained by using the signal enhancement preprocessing (dash line) and then LPSVD analysis (solid line). 
is shown that these criteria in general perform better than those empirically set by the users. Besides, they also help lead to a fully automatic algorithm by minimizing user's involvement during the signal enhancement procedure.

\subsection{Conclusion}

Conceptually, the conventional least-square analysis is also capable of incorporating various types of requirement, constraint, or desideratum. In practice, however, the resulting constrained optimization problem may not be solvable by any known method. The main asset of set theoretic estimation, therefore, stems from the existance of efficient algorithms for finding these solutions and great flexibility with regard to incorporation of all available information. Among various novel algorithms, the feasibility algorithm of iterative parallel projections has demonstrated certain theoretical and computational advantages for practical NMR applications: unrestrictive convergence conditions, its iterative and parallel nature, and attractive convergence behavior. As is previously illustrated by means of examples, a dramatic enhancement in spectral sensitivity and resolution can be obtained at the expense of greater computational complexity. The advent of powerful computers at reasonable cost and the development of fast algorithms should render approaches of this kind usable on a routine basis.

Due to the generality of the set theoretic estimation, this approach can actually be applied to a wide spectrum of NMR and non-NMR applications. For various 


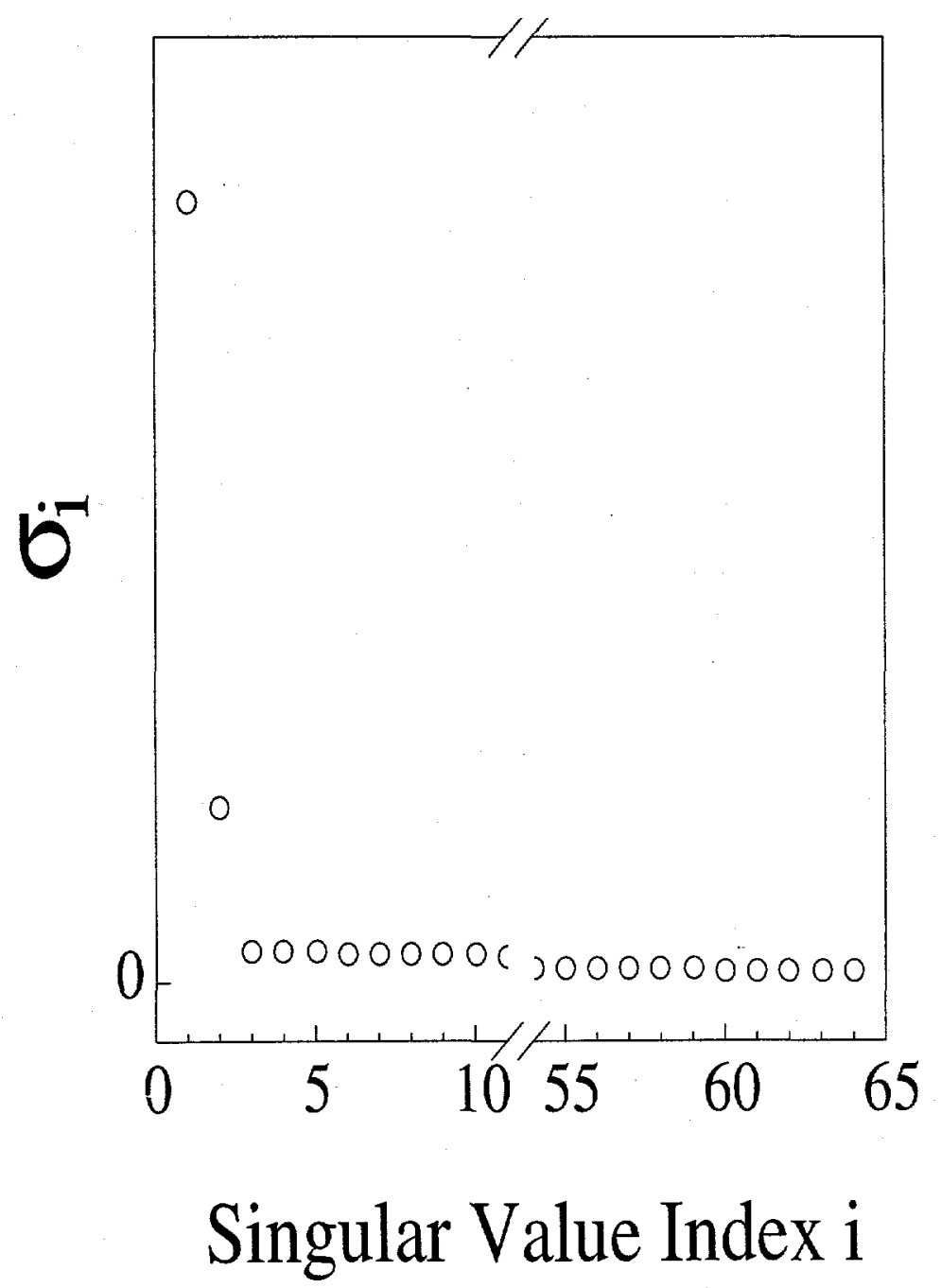

Figure 2.4: The singular values of the data matrix constructed from the FID of $\mathrm{Mg}$ EDTA shown in Fig. 4c. 
situations, one can use different data representations and incorporate different a priori knowledge. Besides the examples shown above, we have also successfully implemented such signal enhancement algorithms in solid-state NMR with non-Lorentzian lineshapes and MRI (not shown). In the solid-state experiments, the inherent $\mathrm{S} / \mathrm{N}$ problem can be aggravated further by long spin lattice relaxation times and relatively broad and complex lineshapes reflecting chemical shift anisotropy and incomplete averaging of the total spin Hamiltonian. Thus, a signal enhancement method without lineshape distortion will be very helpful.

The only way to restrict objectively the feasibility set is to incorporate more information in the formulation. Continuing work will be aimed at incorporating random matrix theory and probabilistic information pertaining to the noise process such as range, moments, second and higher order probabilistic attributes. Such information can be obtained from the tail of the FID where the exact signal has died out, or by acquiring the FID in the absence of a stimulated pulse. The construction of these property sets must resort to statistical confidence limit and fussy set theory. Adding these sets to the collection of sets describing the solution will yield a smaller feasibility set and, hence, more reliable estimates and more significant effect of signal enhancement. It is, therefore, concluded that the true utility of the set theoretic estimation is dependent on the user's theoretical and experimental understanding of the spin system to innovatively introduce signal and noise properties that characterize the underlying applications. 


\section{Bibliography}

[1] P. L. Combettes, Proc. IEEE81, 182 (1993).

[2] J. R. Deller, IEEE ASSP Mag.6, 4 (1989).

[3] H. J. Trussell and M. R. Civanlar, IEEE Trans. Acoust. Speech Signal Process. 32, 201 (1984).

[4] S. M. Kay, Modern Spectral Estimation (Prentice-Hall, Englewood Cliffs, New Jersey, 1987).

[5] S. L. Marple, Digital Spectral Analysis with Applications (Prentice-Hall, Englewood Cliffs, New Jersey, 1987).

[6] N. E. Hurt, Acta Applicandae Mathematicae23, 145 (1991).

[7] S. P. Han, Math. Programming40, 1 (1988).

[8] P. L. Combettes and H. J. Trussell, J. Optimiz. Theory Applications 67, 487 (1990).

[9] G. Crombez, J. Math. Analysis Applications 155, 413 (1991). 
[10] A. R. de Pierro and A. N. Iusem, Appl. Math. Optimiz. 17, 225 (1988).

[11] J. A. Cadzow, IEEE Trans. Acoust. Speech Signal Process. 36, 49 (1988).

[12] J. J. Led and H. Gesmar, Chem. Rev. 91, 1413 (1991).

[13] R. E. Hoffman and G. C. Levy, Prog. NMR Spectrosc. 23, 211 (1991).

[14] H. Gesmar, J. J. Led, and F. Abildgaard, Prog. NMR Spectrosc. 22, 255 (1990).

[15] D. S. Stephenson, Prog. NMR Spectrosc. 20, 515 (1988).

[16] P. L. Combettes, IEEE Trans. Image Process. 2, 269 (1993).

[17] A. N. Iusem and A. R. de Pierro, Math. Programming 52, 265 (1991).

[18] P. L. Combettes, IEEE Trans. Signal Process. 42, 2955 (1994).

[19] W. Zangwill, Nonlinear Programming: A Unified Approach (Prentice-Hall, Englewood Cliffs, New Jersey, 1969).

[20] R. H. Kretsinger, Ann. Rev. Biochem. 45, 239 (1976).

[21] S. Forsén, T. Drakenberg, and H. Wennerstrom, Quart. Rev. Biophys. 19, 83 (1987).

[22] K. J. Neurohr, T. Drakenberg, and S. Forsén, in NMR of Newly Accessible Nuclei, edited by P. Laszlo (Academic Press, New York, 1983), Vol. 2, pp.229-252. 
[23] T. Drakenberg and S. Forsén, in The Multinuclear Approach to NMR Spectroscopy, edited by J. B. Lambert, and F. G. Riddle (Reidel, Dordrecht, 1983), pp. 309-328.

[24] S. Forsén and B. Lindman, Annu. Rep. NMR Spectrosc. 11A, 183 (1981).

[25] R. K. Harris and B. E. Mann (eds.), NMR and the Periodic Table (Academic Press, London, 1978).

[26] D. Tufts and R. Kumaresan, Proc. IEEE 70, 975 (1982).

[27] C. Eckart and G. Young, Psychometrika 1, 211 (1936).

[28] J. A. Cadzow and D. M. Wilkes, Signal Processing 25, 171 (1991).

[29] R. Kumaresan and D. W. Tufts, IEEE Trans. Acoust. Speech Signal Process. 36, 757 (1988).

[30] H. Barkhuijsen, R. de Beer, W. M. M. J. Bovee, and D. van Ormondt, J. Magn. Reson. 61, 465 (1985).

[31] J. J. Led, H. Gesmar, K. R. Hejnaes, and F. B. Hansen, J. Am. Chem. Soc. 110, 4165 (1988).

[32] J. Tang and N. R. Norris, J. Chem. Phys. 84, 5210 (1986).

[33] K. Konstantinides and K. Yao, IEEE Trans. Acoust. Speech Signal Process. 36, 757 (1988). 


\section{Chapter 3}

\section{Matrix Pencil}

\subsection{Abstract}

Many potentially interesting and useful classes of NMR experiments generate data for which conventional spectral estimation and quantification via the Fourier Transform is unsatisfactory. In particular, recently introduced solid-state NMR experiments which involve long delays before data acquisition fall into this category, as the free induction decays are heavily "truncated" and have low signal-to-noise ratios. A novel detection-estimation scheme is introduced in order to analyze data from such experiments and others where the sensitivity is low and/or the data record is strongly damped or truncated. Based on the assumption of exponential data modelling, the number of signals present is first detected using criteria derived from information theory and the spectral parameters are then estimated using the matrix pencil method. 
Monte Carlo simulations and experimental applications are carried out to demonstrate its superior statistical and computational performances and its general applicability to delayed acquisition data. Over the range of noise levels investigated, it is found that this approach is essentially near-optimal in the sense that the estimated spectral parameters having biases almost equal to zero and variances very close to their theoretical Cramér-Rao lower bounds. Compared to the popular method of Linear Prediction with Singular Value Decomposition, this method not only improves the estimation accuracy (by a factor of 2-4) with a lower "breakdown" signal-to-noise threshold $(\approx 1.5 \mathrm{~dB})$, but also reduces the computational cost by about an order of magnitude. It also holds great promise in effectively reducing truncation artifacts. It is concluded that this approach not only facilitates the analysis of delayed acquisition data, but can also become a valuable tool in the routine quantification of general NMR spectra. A listing of programs is also included in the Appendix.

\subsection{Introduction}

Conventional spectral estimation of NMR data is based on the Fourier Transform (FT), which decomposes the time series into a sum of undamped sinusoidal oscillations. This can be done very efficiently using the Fast Fourier Transform. However, the Fourier Transform is only strictly applicable to the limited subset of "complete" signals, i.e., $t=0$ to $\infty[3,4,5,6,7,8,9,10]$. Fourier transformation of signals that are truncated, either at the start or end of the decay, leads to familiar spectral 
distortions, baseline roll and "sinc-wiggles" respectively. Besides, there is no built-in mechanism for noise suppression. The linear nature of the Fourier Transform implies that reducing these problems or improving apparent resolution can only be done at the expense of spectral resolution and/or sensitivity.

The goal of NMR spectral estimation is to obtain an estimate of the frequency response function of the underlying spin system from the measured free induction decay (FID). A particular FID can be characterized in terms of a model function with a set of free parameters. A crucial problem in NMR spectral estimation is, therefore, the detection of the signal model and the estimation of the spectral parameters (e.g., damping factor, frequency, amplitude, and phase). The difficulty of the detectionestimation problem is increased by the low sensitivity inherent in NMR spectroscopy. Because of the computational complexity and noise interference, the problem is usually solved in two steps. The model function is first chosen, and verified on physical grounds or by statistical tests. After successful signal modelling, the free parameters of the signals are then estimated.

Detection theory refers to the selection of the physical or mathematical model that best describes the measured phenomena. The model function must be chosen with care; if the number of parameters is too large, many of them will be spurious, particularly if one has to contend with noise, while too restrictive a model function leads to poor fitting of the data and systematic errors. Estimate statistics are usually better if the number of parameters is minimized. In NMR, the exper- 
imentally observed FID, $\boldsymbol{y}=\left[y_{0}, y_{1}, \ldots, y_{N-1}\right]^{T}$, can be approximated by a sum of complex-valued noise-free signal $\boldsymbol{x}=\left[x_{0}, x_{1}, \ldots, x_{N-1}\right]^{T}$ and additive noise perturbation $\boldsymbol{w}=\left[w_{0}, w_{1}, \ldots, w_{N-1}\right]^{T}$, where " $T$ " denotes matrix transpose and $N$ is the number of complex data points. It is generally assumed that the elements of $w$ are complex Gaussian random variables with zero mean, variance $\rho$, and uncorrelated real and imaginary parts. This a priori assumption of normality is not only mathematically convenient, but via the central limit theorem, it is often a good approximation of the real NMR circumstances.

The quality of spectral estimation can be improved by incorporating further information into the signal model. This is conventionally done by assuming that the signal can be decomposed into a set of exponentially damped oscillations,

$$
\begin{aligned}
y_{n}=x_{n}+w_{n}= & \sum_{i=1}^{M}\left|a_{i}\right| \exp \left(j \theta_{i}\right) \exp \left[\left(-\alpha_{i}+j 2 \pi f_{i}\right) n\right]+w_{n} \\
& =\sum_{i=1}^{M} a_{i} z_{i}^{n}+w_{n} \quad n=0,1, \ldots, N-1
\end{aligned}
$$

where $\left|a_{i}\right|, \alpha_{i}, f_{i}, \theta_{i}$ represent the absolute amplitudes, damping factors (inverse time constants), frequencies, and phases of the $M$ distinct exponentials, respectively; $j$ is used to denote $\sqrt{-1} . z_{i} \equiv \exp \left(-\alpha_{i}+j 2 \pi f_{i}\right)$ is the "signal pole" and $a_{i} \equiv\left|a_{i}\right| \exp \left(j \theta_{i}\right)$ is the "complex amplitude". This is in general a good assumption for liquid-state NMR, and for solid-state NMR with fast magic angle sample spinning[9]. Based on this model function, the detection problem is then reduced to the determination of the 
number of signals $M$. Note that the assumption of exponential decay is not necessarily unduly restrictive. The application of such a model to non-exponentially decaying signals results in a mathematical, rather than a physical, analysis by expanding each spectral component into a sum of exponentials.

Within the last decade, much research activity has been focused on formulating and comparing alternative means of $\mathrm{NMR}$ spectral estimation $[4,5,6,7,8,9,10,10]$, driven by the promise of potentially superior spectral sensitivity and/or resolution in comparison to the conventional Fourier Transform (albeit at the expense of greater computational complexity). In particular, parametric methods based on the exponential FID modelling, cf. Eq. 3.1, have attracted considerable interest. This incorporation of lineshape information should allow individual signals to be better resolved, both from each other and from the noise. In this chapter, a combined detectionestimation scheme, ITMPM, based on Information Theory and Matrix Pencil Method is introduced which improves the estimation performance and computational efficiency of the exponential FID modelling, relative to existing techniques based on Linear Prediction. Monte Carlo simulations are first carried out to verify the statistical superiority of ITMP.M. Its applications to the experimental data from delayed acquisition measurements are then demonstrated. A fully automated program in MATLAB[11] is provided in the Appendix to minimize the user's implementation effort. 


\subsection{Matrix Pencil Method}

There is an important difference between the complex amplitude $a_{i}$ and the signal pole $z_{i}$ in the functional form of Eq. 3.1; the signal model is linear in the first and nonlinear in the second. The complications of nonlinearity can be circumvented by invoking the linear prediction principle [e.g., linear prediction[12, 13], autoregressive modelling[1, 2]] or by employing matrix factorization techniques [e.g., state space formalism[16, 17], matrix pencil method [18, 19, 21]]. In particular, Linear Prediction with Singular Value Decomposition (LPSVD) and related methods have been shown to be useful complements to the Fourier Transform $[4,5,6,7,8,9,10,12,13,22,23$, 24, 25]. The principles of LPSVD have been extensively documented. In summary, one first solves the following linear prediction equations for the coefficients $\left\{c_{i}\right\}_{1 \leq i \leq L}$ of the prediction polynomial,

$$
\left[\begin{array}{cccc}
y_{L-1} & y_{L-2} & \cdots & y_{0} \\
y_{L} & y_{L-1} & \cdots & y_{1} \\
\vdots & \vdots & \ddots & \vdots \\
y_{N-2} & y_{N-3} & \cdots & y_{N-L-1}
\end{array}\right]\left[\begin{array}{c}
c_{1} \\
c_{2} \\
\vdots \\
c_{L}
\end{array}\right]=\left[\begin{array}{c}
y_{L} \\
y_{L+1} \\
\vdots \\
y_{N-1}
\end{array}\right]
$$

The roots of the prediction polynomial $P(z)=1-\sum_{i=1, L} c_{i} z^{-i}$ then produce the $M$ signal poles $\left\{z_{i}\right\}_{1 \leq i \leq M}$. The success of LPSVD resides in the ability of Linear Prediction to extend truncated FIDs for resolution enhancement, or to estimate missing or corrupted initial data points for baseline and phase corrections[26]. The corrupting 
effects of noise can be mitigated through a judicious combination of an over-estimated prediction order $(L \gg M$, introducing extra $L-M$ noise-related poles) to account for the noise in the measurements, and SVD-based signal-subspace techniques to discriminate between signal and noise[10].

While the use of LPSVD for spectral quantification has many advantages, its major drawback is the considerably larger computational burden and higher algorithmic complexity (necessary to avoid numerical instability and overflow) mainly due to the high degree polynomial rooting. In addition, LPSVD exhibits a "breakdown" in estimation performance when noise level increases beyond a certain threshold, e.g., Refs $[4,5,6,7,8,9,10,10,12,13,27]$. These factors make LPSVD less satisfactory, particularly when the signal-to-noise ratio (SNR) is low.

Unlike LPSVD, the recently proposed matrix pencil method, developed independently by Hua and Sarkar[18, 19] and by Kailath et al. [21], involves finding the signal poles, $z_{i}$, directly by solving a generalized eigenvalue problem. Its formulation is governed by the notion of pencil-of-functions and exploits the property of a matrix pencil constructed from the underlying FID. The mathematical entity "matrix pencil" refers to the linear combination of two matrices (say, $\boldsymbol{F}$ and $\boldsymbol{G}$ ) defined on a common domain, i.e., $\boldsymbol{F}+\lambda \boldsymbol{G}$. The eigenvalues of the matrix pencil are defined as the values of the scalar variable $\lambda$ that decrease the rank of the matrix pencil.

Let $\boldsymbol{X}_{0}$ and $\boldsymbol{X}_{1}$ be two noise-free data matrices with dimension $(N-L) \times L$ 
defined by

$$
\boldsymbol{X}_{0}=\left[\begin{array}{cccc}
x_{L-1} & x_{L-2} & \cdots & x_{0} \\
x_{L} & x_{L-1} & \cdots & x_{1} \\
\vdots & \vdots & \ddots & \vdots \\
x_{N-2} & x_{N-3} & \cdots & x_{N-L-1}
\end{array}\right], \boldsymbol{X}_{1}=\left[\begin{array}{cccc}
x_{L} & x_{L-1} & \cdots & x_{1} \\
x_{L+1} & x_{L} & \cdots & x_{2} \\
\vdots & \vdots & \ddots & \vdots \\
& & & \\
x_{N-1} & x_{N-2} & \cdots & x_{N-L}
\end{array}\right]
$$

where $L$ is called the pencil parameter. It follows from Eq. 3.1 that these matrices can be decomposed as

$$
X_{0}=Z_{L} B Z_{R}, \quad X_{1}=Z_{L} B Z Z_{R}
$$


where

$$
\begin{gathered}
Z_{L}=\left[\begin{array}{ccccc}
1 & 1 & \cdots & 1 \\
z_{1} & z_{2} & \cdots & z_{M} \\
\vdots & \vdots & \ddots & \vdots \\
z_{1}{ }^{N-L-1} & z_{2}{ }^{N-L-1} & \cdots & z_{M}^{N-L-1}
\end{array}\right], \boldsymbol{B}=\left[\begin{array}{cccc}
a_{1} & 0 & \cdots & 0 \\
0 & a_{2} & \cdots & 0 \\
\vdots & \vdots & \ddots & \vdots \\
0 & 0 & \cdots & a_{M}
\end{array}\right], \\
Z_{R}=\left[\begin{array}{cccc}
z_{1}^{L-1} & z_{1}{ }^{L-2} & \cdots & 1 \\
z_{2}{ }^{L-1} & z_{2}{ }^{L-2} & \cdots & 1 \\
\vdots & \vdots & \ddots & \vdots \\
z_{M}{ }^{L-1} & z_{M}{ }^{L-2} & \cdots & 1
\end{array}\right],
\end{gathered}
$$

$Z_{L}$ and $Z_{R}$ are Vandermonde matrices, and $B$ and $Z$ are diagonal matrices constructed from the complex amplitudes and signal poles respectively.

Now consider the matrix pencil $\boldsymbol{X}_{1}-\lambda \boldsymbol{X}_{0}$

$$
\boldsymbol{X}_{1}-\lambda \boldsymbol{X}_{0} \quad=\boldsymbol{Z}_{L} \boldsymbol{B}\left[\begin{array}{cccc}
=\boldsymbol{Z}_{L} \boldsymbol{B}\left(\boldsymbol{Z}-\lambda \boldsymbol{I}_{M}\right) \boldsymbol{Z}_{R} & \\
z_{1}-\lambda & 0 & \cdots & 0 \\
0 & z_{2}-\lambda & \cdots & 0 \\
\vdots & \vdots & \ddots & \vdots \\
0 & 0 & \cdots & z_{M}-\lambda
\end{array}\right] \boldsymbol{Z}_{R}
$$


where $\boldsymbol{I}_{M}$ is an $M \times M$ identity matrix. In general, the rank of the matrix pencil $\boldsymbol{X}_{1}-\lambda \boldsymbol{X}_{0}$ is $M$. However, if $\lambda=z_{i}$ then the rank of $\boldsymbol{Z}-\lambda \boldsymbol{I}_{M}$ will be reduced to $M-1$. In other words, each of the $z_{i}$ will be a rank reducing number of the matrix pencil $\boldsymbol{X}_{1}-\lambda \boldsymbol{X}_{0}$, and so, by definition, the set $\left\{z_{i}\right\}_{1 \leq i \leq M}$ can be identified with the $M$ non-zero generalized eigenvalues of the matrix pair $\left(\boldsymbol{X}_{1}, \boldsymbol{X}_{0}\right)$,

$$
\boldsymbol{X}_{\mathbf{1}} \boldsymbol{q}_{i}=z_{i} \boldsymbol{X}_{0} \boldsymbol{q}_{i}
$$

where $\boldsymbol{q}_{i}$ is the eigenvector associated with the eigenvalue (and signal pole) $z_{i}$. The Moore-Penrose pseudo-inverse of a matrix is a generalization of the matrix inverse to the case where the matrix is not square and possibly of incomplete rank. Left multiplying Eq. 3.9 by the Moore-Penrose pseudo-inverse of $\boldsymbol{X}_{0}, \boldsymbol{X}_{0} \#$, and using the property that $\boldsymbol{X}_{0}{ }^{\#} \boldsymbol{X}_{0}=\boldsymbol{I}$, it is clear that the generalized eigenvalues of $\left(\boldsymbol{X}_{1}, \boldsymbol{X}_{0}\right)$ can be solved by finding the $M$ non-zero eigenvalues of the $L \times L$ matrix product $\boldsymbol{X}_{0}^{\#} \boldsymbol{X}_{1}$,

$$
\boldsymbol{X}_{0}^{\#} \boldsymbol{X}_{1} \boldsymbol{q}_{i}=z_{i} \boldsymbol{q}_{i}
$$

In order to apply such techniques to experimental data, it is important to account for the effects of noise corruption. In spectral estimation, the data matrix, covariance matrix, and autocorrelation matrix characterize the information contained in the observed signal. When constructed from noiseless signal, these matrices possess cer- 
tain eigen-characteristics (e.g., rank, degeneracy, positive semidefinite, etc.) and/or matrix structures (e.g., Hermitian, Hankel, Toeplitz, etc.). Inevitable measurement noise, however, results in the loss of part (or all) of these theoretical matrix properties. Provided the noise perturbation is not too large, a useful procedure for approximating the noiseless matrix is to find the matrix which possesses a set of chosen properties and lies closest to the empirical noisy matrix. The resulting matrix is a more accurate representation of the characteristics of the underlying signal than the original noisy matrix, where the corrupting effect of noise has been mitigated and a signal enhancement has been accomplished.

This can be successfully incorporated into the matrix pencil method. For the noisy data, we define $\boldsymbol{Y}_{0}$ and $\boldsymbol{Y}_{1}$ the same way as for $\boldsymbol{X}_{0}$ and $\boldsymbol{X}_{1}$ with $x_{i}$ replaced by the noisy measurement $y_{i}$. It is clear that, while the noiseless daia matrices $\boldsymbol{X}_{0}$ and $\boldsymbol{X}_{1}$ have a rank equal to the number of signal components $(M)$, the noisy data matrices, $Y_{0}$ and $Y_{1}$, will in general be of full rank due to noise contamination. Singular Value Decomposition (SVD) provides a particularly useful tool for restoring the matrixrank property. SVD, one of the most stable and computationally effective algorithm in the theory of matrix algebra, is a generalization of the eigenvalue decomposition for non-square matrices[10, 28, 1, 2]. The SVD theorem states that for an arbitrary $(N-L) \times L$ matrix $\boldsymbol{Y}_{0}$ there exist positive real numbers $\sigma_{1} \geq \sigma_{2} \geq \cdots \geq \sigma_{R}>0$ (the so-called singular values), an $(N-L) \times(N-L)$ unitary matrix $U=\left[u_{1} u_{2} \cdots u_{N-L}\right]$, 
and an $L \times L$ unitary matrix $V=\left[\boldsymbol{v}_{1} \boldsymbol{v}_{2} \cdots \boldsymbol{v}_{L}\right]$ such that

$$
\boldsymbol{Y}_{0}=U\left[\begin{array}{c}
\Sigma \\
0
\end{array}\right] \boldsymbol{V}^{\dagger}=\sum_{i=1}^{R} \sigma_{i} \boldsymbol{u}_{i} \boldsymbol{v}_{i}^{\dagger}=\sigma_{1} u_{1} v_{1}^{\dagger}+\sigma_{2} u_{2} v_{2}^{\dagger}+\cdots+\sigma_{R} u_{R} v_{R}^{\dagger}
$$

where $\boldsymbol{\Sigma}=\operatorname{diag}\left(\sigma_{1}, \sigma_{2}, \cdots, \sigma_{R}\right)$ is an $R \times R$ diagonal matrix, $\mathbf{0}$ is a null matrix, "†" denotes conjugate transpose, and $R \leq \min (N-L, L)$ is defined as the rank of $Y_{0}$. The matrix $Y_{0}$ is thus constructed from $R$ rank-one matrices weighted by their respective singular values. The matrix of rank $M(<R)$ which lies closest, in a least squares sense, to the original matrix is constructed using the first $M$ principle singular values and the associated singular vectors [Eckard-Young theorem[29]], i.e.,

$$
\widehat{\boldsymbol{Y}_{0}}=U_{M} \boldsymbol{\Sigma}_{M} \boldsymbol{V}_{M}^{\dagger}=\sum_{i=1}^{M} \sigma_{i} \boldsymbol{u}_{i} \boldsymbol{v}_{i}^{\dagger}
$$

where $\boldsymbol{U}_{M}=\left[\boldsymbol{u}_{1} \boldsymbol{u}_{2} \cdots \boldsymbol{u}_{M}\right], \boldsymbol{V}_{M}=\left[\boldsymbol{v}_{1} \boldsymbol{v}_{2} \cdots \boldsymbol{u}_{M}\right]$, and $\boldsymbol{\Sigma}_{M}=\operatorname{diag}\left(\sigma_{1}, \sigma_{2}, \cdots, \sigma_{M}\right)$.

The Moore-Penrose pseudo-inverse can also be defined in terms of the SVD components of $Y_{0}$,

$$
{\widehat{Y_{0}}}^{\#}=V_{M} \boldsymbol{\Sigma}_{M}^{-1} U_{M}^{\dagger}=\sum_{i=1}^{M} \sigma_{i}^{-1} v_{i} \boldsymbol{u}_{i}^{\dagger}
$$

Hence, for noisy data, the $M$ non-zero eigenvalues of the signal-enhanced $L \times L$ matrix product ${\widehat{Y_{0}}}^{\#} \boldsymbol{Y}_{1}$ give the estimates of the signal poles $z_{i}$, and hence the damping factors $\alpha_{i}=-\log \left|z_{i}\right|$, and frequencies $f_{i}=\arg \left(z_{i}\right) / 2 \pi$. It should be noted that 
further replacement of $\boldsymbol{Y}_{1}$ by its $\operatorname{rank} M$ counterpart $\widehat{\boldsymbol{Y}_{1}}$ offers little advantage, due to the strong correlation of the noise between $\boldsymbol{Y}_{0}$ and $\boldsymbol{Y}_{1}$. Once $\left\{z_{i}\right\}_{1 \leq i \leq M}$ are known, the absolute amplitudes $\left|a_{i}\right|$ and phases $\theta_{i}=\arg \left(a_{i}\right)$ can be solved by a general linear least-squares analysis

$$
\left[\begin{array}{c}
y_{0} \\
y_{1} \\
\vdots \\
y_{N-1}
\end{array}\right]=\left[\begin{array}{cccc}
1 & 1 & \cdots & 1 \\
z_{1} & z_{2} & \cdots & z_{M} \\
\vdots & \vdots & \ddots & \vdots \\
z_{1}^{N-1} & z_{2}{ }^{N-1} & \cdots & z_{M}^{N-1}
\end{array}\right]\left[\begin{array}{c}
a_{1} \\
a_{2} \\
\vdots \\
a_{M}
\end{array}\right]+\left[\begin{array}{c}
w_{0} \\
w_{1} \\
\vdots \\
w_{N-1}
\end{array}\right]
$$

Note that the pencil parameter $L$ (where $M<L<N$ ) will influence the quality of the results, in a similar way that LPSVD is affected by the choice of the polynomial degree (prediction order). A poor choice of prediction order or pencil parameter will limit the performance of either technique. It is empirically found that the optimal value for $L$ ranges from $L=N / 3$ for noisy signals, to $L=N / 2$ for signals with a higher SNR[18, 19].

The computational efficiency can be increased by noting that since ${\widehat{Y_{0}}}^{\#} \boldsymbol{Y}_{1}$ has rank $M<L, L-M$ of its eigenvalues are zero[19]. The size of $\widehat{\boldsymbol{Y}}_{0}^{\#} \boldsymbol{Y}_{1}$ can therefore be reduced before its eigenvalues are found. Substituting for $\widehat{Y}_{0}^{\#}$ from Eq. 3.13 into the eigenvalue equation, Eq. 3.10, for ${\widehat{Y_{0}}}^{\#} \boldsymbol{Y}_{1}$,

$$
V_{M} \Sigma_{M}^{-1} U_{M}^{\dagger} \boldsymbol{Y}_{1} \boldsymbol{q}_{i}=z_{i} \boldsymbol{q}_{i}
$$


Left multiplying by $V_{M}{ }^{\dagger}$ and using the unitary nature of $V_{M}$, i.e., $V_{M} V_{M}^{\dagger}=$ $V_{M}^{\dagger} V_{M}=I$, we have

$$
\boldsymbol{\Sigma}_{M}^{-1} \boldsymbol{U}_{M}^{\dagger} \boldsymbol{Y}_{1} \boldsymbol{V}_{M}\left(\boldsymbol{V}_{M}^{\dagger} \boldsymbol{q}_{i}\right)=z_{i}\left(\boldsymbol{V}_{M}^{\dagger} \boldsymbol{q}_{i}\right)
$$

Now it can be seen that the estimates of $\left\{z_{i}\right\}_{1 \leq i \leq M}$ can be found by computing the eigenvalues of the much smaller $M \times M$ matrix $\boldsymbol{\Sigma}_{M}{ }^{-1} U_{M}^{\dagger} \boldsymbol{Y}_{1} V_{M}$.

\subsection{Information Theory}

A potential difficulty encountered in practical applications of matrix pencil and other SVD-based methods is the ambiguity in detecting the number of signal components, that is, in choosing the matrix rank $M[30]$. The determination of $M$ is crucial; too small a value of $M$ results in information loss, while too large a value effectively incorporates more noise and generates spurious spectral features. For well-resolved spectra with reasonable SNR, the value of $M$ can be determined from the sharp cutoff in magnitude of the singular values, or the number of resolved peaks in the FT spectrum above a predefined threshold. These criteria, however, become ill-defined as the spacing between resonance frequencies, the number of data points, or the SNR decreases.

Various criteria have been proposed to address this problem. They may be classified into the following five categories: (i) subjective threshold settings of singular 
values[28, 31, 32], e.g., $\sigma_{1} \geq \sigma_{2} \geq \cdots \geq \sigma_{M}>\delta \geq \sigma_{M+1}$, where the threshold value $\delta$ is selected on an ad hoc basis; (ii) statistical threshold bounds on singular values [30], which is similar to (i) but the threshold is based on the theories of perturbations of singular values and statistical significance test; (iii) hypothesis test of likelihood ratios $[33,34]$, for each hypothesis the likelihood ratio statistic is compared to a subjective threshold level; (iv) matching of reconstruction residue with noise power[35, 36], consecutive reconstructions for various rank are performed and the resulting error power is compared to the noise power; (v) information theory for model order selection[37]. All these criteria appear intuitively reasonable and function effectively over various cases. Moreover, the information theoretic criteria, (v), have certain theoretical and computational advantages over the others. These criteria were originally introduced in the context of linear prediction by Akaike [AIC, Akaike Information Criterion[38]] and by Schwartz and Rissanen [MDL, Minimum Description Length[39, 40]], and later adapted to exponential modelling by Wax and Kailath[37]. Unlike the conventional approaches in categories (i) and (iii), AIC and MDL do not require any subjective threshold settings. Statistically determined criteria generally perform better than those empirically set by the user, as well as minimizing the necessity for user involvement. The principle and the derivation of these criteria can be found elsewhere[1,2], but in summary, the optimal value of $M$ is determined merely by minimizing a discrete function of the singular values (which are already known 
from Eq. 3.11), e.g.,

$$
\operatorname{MDL}(k)=-\log \left[\frac{\left(\prod_{i=k+1}^{L} \sigma_{i}\right)^{N}}{\left(\frac{1}{L-k} \sum_{i=k+1}^{L} \sigma_{i}\right)^{(L-k) N}}\right]+\frac{1}{2} k(2 L-k) \log (N)
$$

This is very different from the approaches in category (iv) where a priori knowledge of the noise power and extra computational cost for consecutive reconstructions are definitely required. In this work, MDL is used in preference to AIC as it has been shown to give consistent estimates of $M$, while AIC tends to overestimate the number of signals as the number of data points increases[37].

The flowchart for the ITMPM algorithm is depicted in Fig. 3.1.

\subsection{Monte Carlo Simulations}

The development of any signal processing protocol requires a rigorous statistical evaluation of its performance. Visual comparison of the results of a few realizations is insufficient to draw any general conclusions $[1,2]$. The bias, variance, and mean relative error (MRE) are statistical measures that are commonly used to quantify the performance:

$$
\begin{gathered}
\operatorname{bias}(\hat{\beta}) \equiv \varepsilon(\hat{\beta})-\beta \\
\operatorname{var}(\hat{\beta}) \equiv \varepsilon\left\{[\hat{\beta}-\varepsilon(\hat{\beta})]^{2}\right\} \\
\operatorname{MRE}(\hat{\beta}) \equiv \varepsilon\left(\left|\frac{\hat{\beta}-\beta}{\beta}\right|\right) \times 100 \%
\end{gathered}
$$




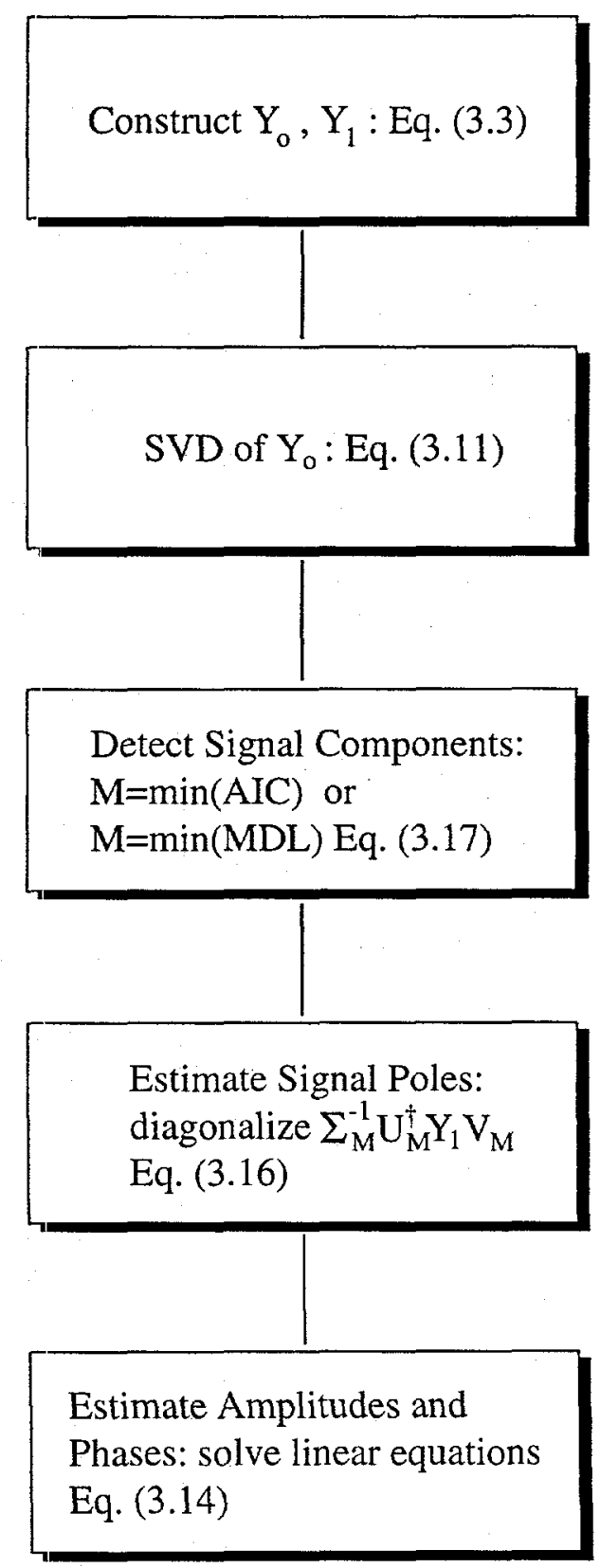

Figure 3.1: The flowchart of the ITMPM algorithm. 
where $\varepsilon$ is the expectation operator and $\hat{\beta}$ is the estimate of the spectral parameter whose true value is $\beta$. Ideally the bias should be negligibly small and the variance as close as possible to its theoretical lower limit, the Cramér-Rao Lower Bound (see below).

Analytical determination of these functions is generally intractable. Instead they can be estimated using Monte Carlo simulations in which the statistics are calculated using the same noise-free data set, but many different realizations of the noise. In this section, three simulation experiments are carried out to evaluate the performance of ITMPM, while LPSVD is used as a benchmark against which ITMPM can be compared. The MATLAB code for both ITMPM and the original Kumaresan-Tufts' LPSVD algorithm with bias compensation[12, 13] is listed in the Appendix. The signal-to-noise ratio (SNR) is here defined as

$$
\mathrm{SNR} \equiv 10 \log _{10} \frac{\sum_{i}\left|a_{i}\right|^{2}}{\rho}(\mathrm{dB})
$$

where $\rho$ is the noise variance.

Example I: Single Exponentially Damped Sinusoid. In this example, the synthesized FIDs are given by $y_{n}=|a| \exp (j \theta) \exp [(-\alpha+j 2 \pi f) n]+w_{n}$ with damping factor $\alpha=0.1$, normalized frequency $f=0.52$, absolute amplitude $|a|=1.0$, phase $\theta=0$, and $n=0, \ldots, 24$. For non-parametric estimators (e.g., the Fourier Transform), the noise can be observed directly in the spectrum, while for parametric 
estimators (e.g., LPSVD and ITMPM), the noise manifests itself as uncertainty in the estimated parameters, leading to a distribution of observed parameters values. Ideally the mean of this distribution corresponds to the true value of the parameter, i.e., the estimation method is unbiased, while the minimum value of its standard deviation is given by the Cramér-Rao Lower Bound (CRLB), also known as the Minimum Variance Bound $[1,2]$. The calculation of these lower bounds is reasonably straightforward for signals distorted by uncorrelated Gaussian noise and parallels exactly the calculation of the covariance matrix for least-squares model-fitting; indeed the CRLBs are identical to the standard deviations on parameters values returned by model-fitting (in the absence of systematic error). The details of the calculation can be found elsewhere[12, 19, 20].

Table 1 summaries the results of a Monte Carlo simulation using 500 noise realizations for each value of the SNR, using $M=1$. It can be seen that the standard deviations (square root of the variance) for ITMPM are close to the theoretical lower bounds, and, unlike LPSVD, the bias on each ITMPM result is always much smaller than its standard deviation. For all SNRs tested here, ITMPM is more accurate and precise than LPSVD. 
Table 3.1: Bias \pm Standard Deviation of the Estimated Spectral Parameters

\begin{tabular}{|rlrrrr|}
\hline \multirow{2}{*}{ SNR (dB) } & Method & Damping Factor $\alpha$ & Frequency $f$ & Amplitude $|a|$ & Phase $\theta$ \\
\hline \multirow{2}{*}{50} & & & & & \\
& CRLB & $0 \pm 2.10 \mathrm{E}-4$ & $0 \pm 3.35 \mathrm{E}-5$ & $0 \pm 1.32 \mathrm{E}-3$ & $0 \pm 1.32 \mathrm{E}-3$ \\
& ITMPM & $-7.34 \mathrm{E}-7 \pm 2.38 \mathrm{E}-4$ & $-1.30 \mathrm{E}-6 \pm 3.63 \mathrm{E}-5$ & $1.69 \mathrm{E}-5 \pm 1.41 \mathrm{E}-3$ & $2.74 \mathrm{E}-5 \pm 1.37 \mathrm{E}-3$ \\
& LPSVD & $5.83 \mathrm{E}-4 \pm 2.69 \mathrm{E}-4$ & $-1.21 \mathrm{E}-6 \pm 3.93 \mathrm{E}-5$ & $2.55 \mathrm{E}-3 \pm 1.53 \mathrm{E}-3$ & $2.50 \mathrm{E}-5 \pm 1.43 \mathrm{E}-3$ \\
40 & CRLB & $0 \pm 6.65 \mathrm{E}-4$ & $0 \pm 1.06 \mathrm{E}-4$ & $0 \pm 4.18 \mathrm{E}-3$ & $0 \pm 4.18 \mathrm{E}-3$ \\
& ITMPM & $-9.99 \mathrm{E}-6 \pm 7.37 \mathrm{E}-4$ & $6.93 \mathrm{E}-6 \pm 1.13 \mathrm{E}-4$ & $-2.71 \mathrm{E}-4 \pm 4.58 \mathrm{E}-3$ & $-3.05 \mathrm{E}-4 \pm 4.16 \mathrm{E}-3$ \\
& LPSVD & $1.86 \mathrm{E}-3 \pm 8.35 \mathrm{E}-4$ & $5.56 \mathrm{E}-6 \pm 1.21 \mathrm{E}-4$ & $7.77 \mathrm{E}-3 \pm 4.84 \mathrm{E}-3$ & $-2.68 \mathrm{E}-4 \pm 4.38 \mathrm{E}-3$ \\
30 & CRLB & $0 \pm 2.10 \mathrm{E}-3$ & $0 \pm 3.35 \mathrm{E}-4$ & $0 \pm 1.32 \mathrm{E}-2$ & $0 \pm 1.32 \mathrm{E}-2$ \\
& ITMPM & $1.57 \mathrm{E}-4 \pm 2.21 \mathrm{E}-3$ & $8.82 \mathrm{E}-6 \pm 3.50 \mathrm{E}-4$ & $9.26 \mathrm{E}-4 \pm 1.40 \mathrm{E}-2$ & $-5.81 \mathrm{E}-4 \pm 1.31 \mathrm{E}-2$ \\
& LPSVD & $5.98 \mathrm{E}-3 \pm 2.53 \mathrm{E}-3$ & $1.02 \mathrm{E}-5 \pm 3.85 \mathrm{E}-4$ & $2.55 \mathrm{E}-2 \pm 1.50 \mathrm{E}-2$ & $-6.18 \mathrm{E}-4 \pm 1.38 \mathrm{E}-2$ \\
24 & CRLB & $0 \pm 4.19 \mathrm{E}-3$ & $0 \pm 6.68 \mathrm{E}-4$ & $0 \pm 2.64 \mathrm{E}-2$ & $0 \pm 2.64 \mathrm{E}-2$ \\
& ITMPM & $2.44 \mathrm{E}-4 \pm 4.40 \mathrm{E}-3$ & $6.46 \mathrm{E}-5 \pm 7.01 \mathrm{E}-4$ & $1.99 \mathrm{E}-3 \pm 2.71 \mathrm{E}-2$ & $-2.44 \mathrm{E}-3 \pm 2.71 \mathrm{E}-2$ \\
& LPSVD & $1.17 \mathrm{E}-2 \pm 5.42 \mathrm{E}-3$ & $5.70 \mathrm{E}-5 \pm 7.68 \mathrm{E}-4$ & $4.89 \mathrm{E}-2 \pm 2.92 \mathrm{E}-2$ & $-2.21 \mathrm{E}-3 \pm 2.85 \mathrm{E}-2$ \\
18 & CRLB & $0 \pm 8.37 \mathrm{E}-3$ & $0 \pm 1.33 \mathrm{E}-3$ & $0 \pm 5.26 \mathrm{E}-2$ & $0 \pm 5.26 \mathrm{E}-2$ \\
& ITMPM & $2.69 \mathrm{E}-3 \pm 9.74 \mathrm{E}-3$ & $-2.54 \mathrm{E}-5 \pm 1.35 \mathrm{E}-3$ & $1.66 \mathrm{E}-2 \pm 5.52 \mathrm{E}-2$ & $4.88 \mathrm{E}-4 \pm 5.54 \mathrm{E}-2$ \\
& LPSVD & $2.52 \mathrm{E}-2 \pm 1.26 \mathrm{E}-2$ & $-3.22 \mathrm{E}-5 \pm 1.49 \mathrm{E}-3$ & $1.02 \mathrm{E}-1 \pm 5.92 \mathrm{E}-2$ & $5.83 \mathrm{E}-4 \pm 5.65 \mathrm{E}-2$ \\
\hline
\end{tabular}


There are a couple of reasons why ITMPM can perform better than LPSVD $[18,19,41,42]$. Despite their differences, both methods solve the following matrix prediction equation

$$
\widehat{Y_{0}} C=Y_{1}
$$

The solution obtained by ITMPM is

$$
{\widehat{\boldsymbol{Y}_{0}}}^{\#} \boldsymbol{Y}_{1}=\left[\widehat{\boldsymbol{Y}}_{0}^{\#} \boldsymbol{y}_{L}, \widehat{\boldsymbol{Y}}_{0}^{\#} \boldsymbol{y}_{L-1}, \cdots, \widehat{\boldsymbol{Y}}_{0}^{\#} \boldsymbol{y}_{1}\right]
$$

where $\boldsymbol{y}_{i}=\left[y_{i}, y_{i+1}, \cdots, y_{i+N-L-1}\right]^{T}(i=1,2, \cdots, L)$. Each column of $C_{\text {ITMPM }}$ (say, the $j$ th column) is a solution to the equation

$$
\widehat{Y_{0}} c=y_{L-j+1} .
$$

Note that, as $\widehat{Y_{0}}$ has a nontrivial null space, the least squares solution to Eq. 3.24 is not unique. Of all the least squares solution, the one with the minimum Euclidean norm is unique and is given by $\widehat{\boldsymbol{Y}}_{0}^{\#} \boldsymbol{y}_{L-j+1}$. The minimum-norm choice has been shown to be an effective way to overcome the estimate sensitivity to noise perturbation[43]. On the other hand, the solution provided by LPSVD is simply 
(cf. Eq. 3.3)

$$
\boldsymbol{C}_{\mathrm{LPSVD}}=\left[\widehat{\boldsymbol{Y}}_{0}^{\#} \boldsymbol{y}_{L}, \boldsymbol{J}_{L}, \boldsymbol{J}_{L-1}, \cdots, \boldsymbol{J}_{2}\right]
$$

where $J_{i}$ is the $L \times 1$ vector with its $i$ th element equal to 1 and all other elements zero. It is clear from Eq. 3.25 that only the first column of $C_{\text {LPSVD }}$ is the minimumnorm solution to Eq. 3.24 while the other columns are just trivial solutions. $C_{\text {ITMPM }}$ however, is the unique minimum-norm solution to the full matrix prediction equation of Eq. 3.22; this results in better immunity to noise perturbation.

In addition, ITMPM distinguishes more reliably between signal and noise eigenvalues (poles). This is because $C_{\text {ITMPM }}$ has $M$ eigenvalues at $\left\{z_{i}\right\}_{1 \leq i \leq M}$ and $L-M$ extraneous zero eigenvalues, while $\boldsymbol{C}_{\mathrm{LPSVD}}$ has $M$ eigenvalues at $\left\{z_{i}\right\}_{1 \leq i \leq M}$ and $L-M$ extraneous eigenvalues that are nonzero and located inside the unit circle. (Recall that $C_{\mathrm{LPSVD}}$ is in fact the companion matrix of the prediction polynomial in LPSVD, and solving the roots of a polynomial is equivalent to solving the eigenvalues of its companion matrix.) Consequently, LPSVD requires a tedious pruning step in discriminating the signal eigenvalues from the extraneous ones introduced by the noise. This discrimination becomes increasingly difficult as the noise level increases.

Example II: ${ }^{19} \mathrm{~F}$ Spectrum of p-fluorophenol. Figure 3.2 (a) shows the theoretical ${ }^{19} \mathrm{~F}$ NMR spectrum of p-fluorophenol, a heteronuclear system containing 5 spin-1/2 species. The theoretical FIDs used in Example II and Example III are calcu- 
lated by the NMR computer simulation package GAMMA[1]. The equilibrium density matrix of the underlying spin system is first treated by a $(\pi / 2)_{y}$ ideal pulse, and then propagated in time governed by the isotropic chemical shift and scalar coupling Hamiltonian without relaxation effects. The FID, taken by a single-quantum quadrature detection operator sampling the $x y$-magnetization, is apodized by an exponential decay function to simulate $\mathrm{T}_{2}$ relaxation. Fig. 3.2 (b) shows the FT spectrum of a typical FID used in the Monte Carlo simulation (SNR $=14.5 \mathrm{~dB}$ ) while $(\mathrm{c})$ is the corresponding ITMPM spectrum. The improvement in sensitivity is substantial, although the effects of noise can still be seen as small errors in the phases of the lines as compared to the ideal spectrum, (a).

The results of Monte Carlo simulations using 400 noise realizations for various SNR with $M=9$ are shown in Fig. 3.3. Figs. 3.3 (b) and (c) show the mean relative errors in the damping factors and amplitudes respectively; both ITMPM and LPSVD give very reliable estimates of signal frequencies[44]. Signals may not be correctly detected at low SNRs; for this example, detection is defined as successful if all the peaks are resolved within $\pm 0.6 \mathrm{ppm}$ (about $1 / 6$ of the average peak spacing) of their correct chemical shifts. Fig. 3.3 (a) shows the failure rate as a function of SNR. It is apparent that ITMPM not only provides significantly more accurate estimates of the spectral parameters (by a factor of 2-4), but also has a lower SNR "breakdown" threshold (lower by $\approx 1.5 \mathrm{~dB}$, as estimated from the success rate).

In practice, the usefulness of "exotic" spectral estimation methods, whatever their 

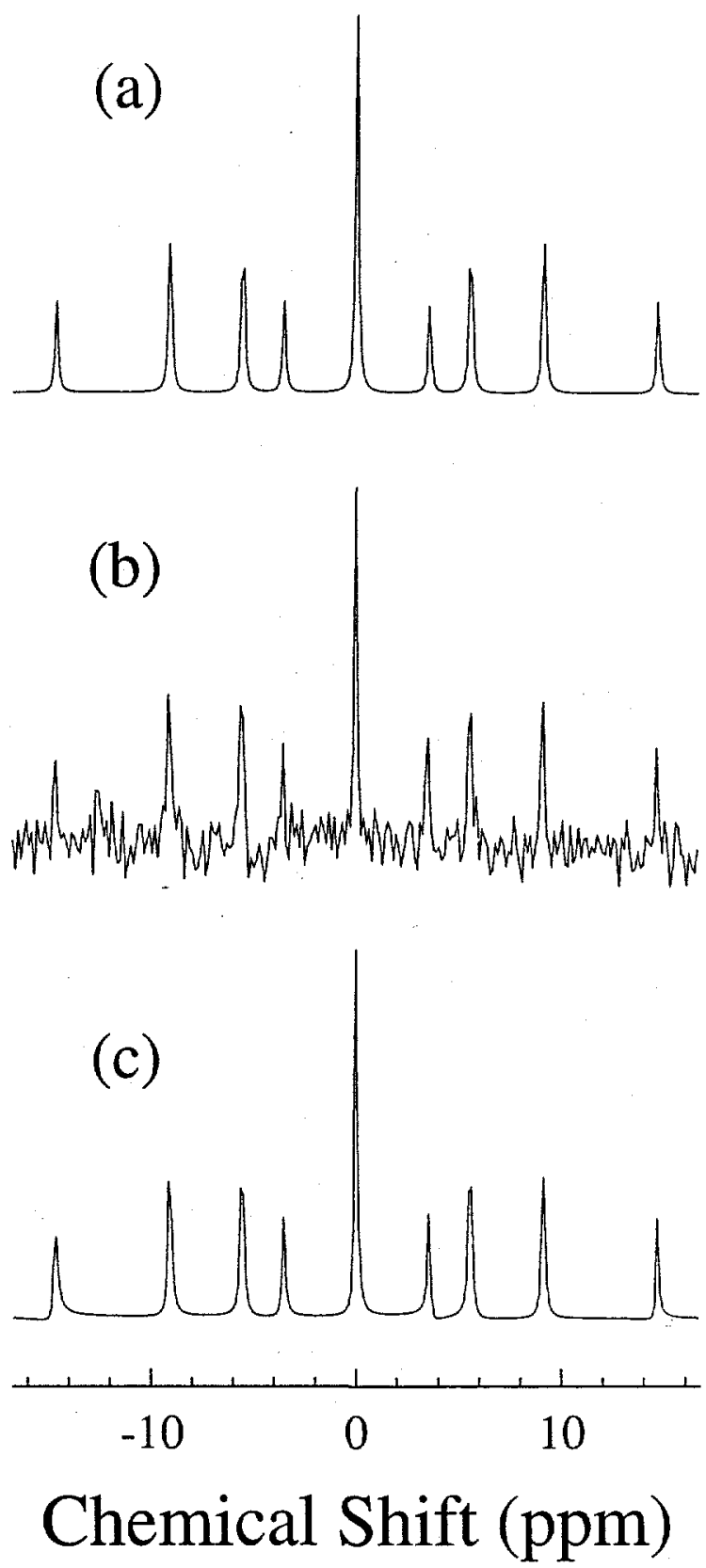

Figure 3.2: The ${ }^{19} \mathrm{~F}$ spectrum of p-fluorophenol calculated assuming weak coupling. (a) FT spectrum of the noiseless FID; (b) FT spectrum of a FID with SNR $=14.5$ $\mathrm{dB}$; (c) ITMPM spectrum of (b). 

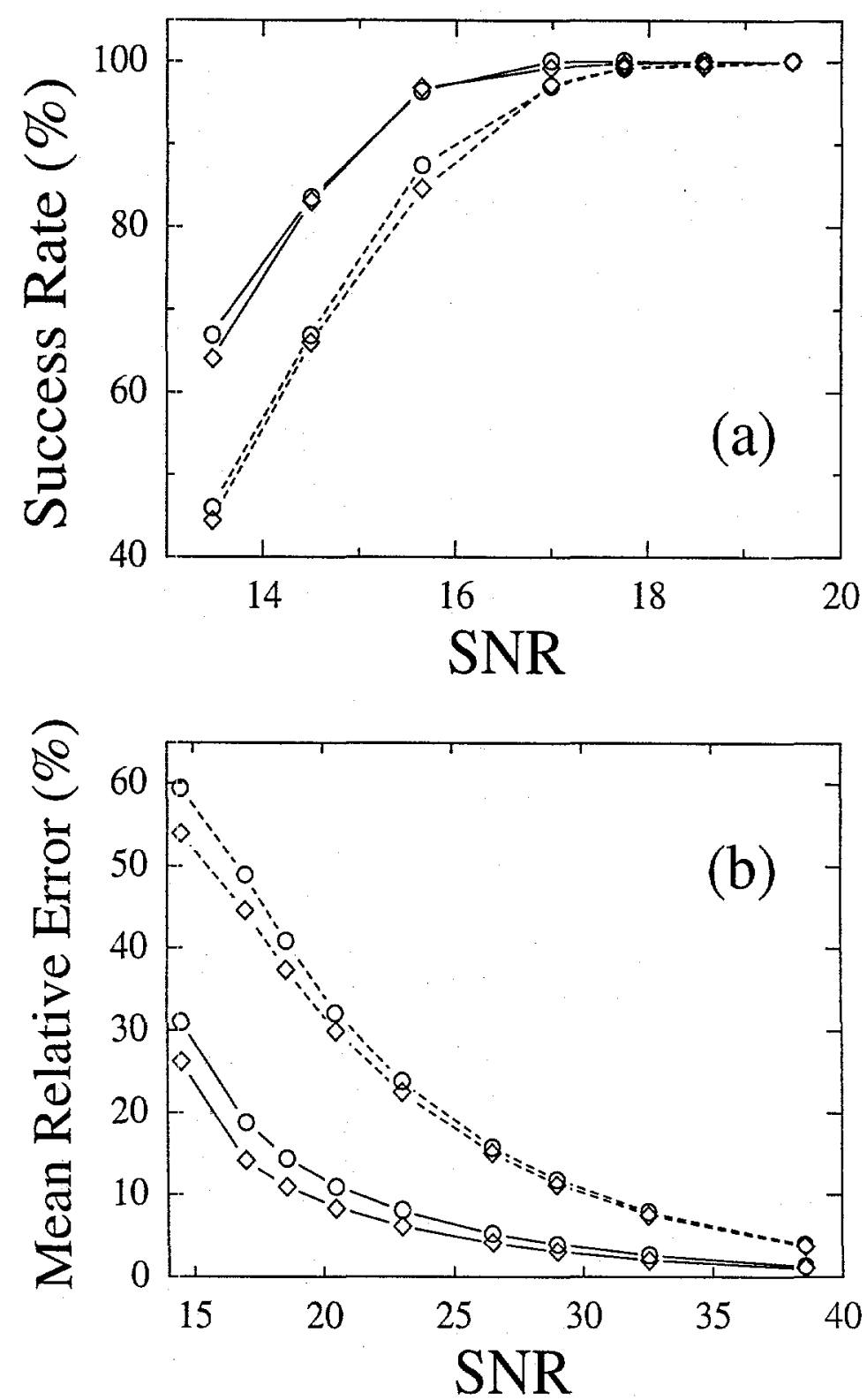


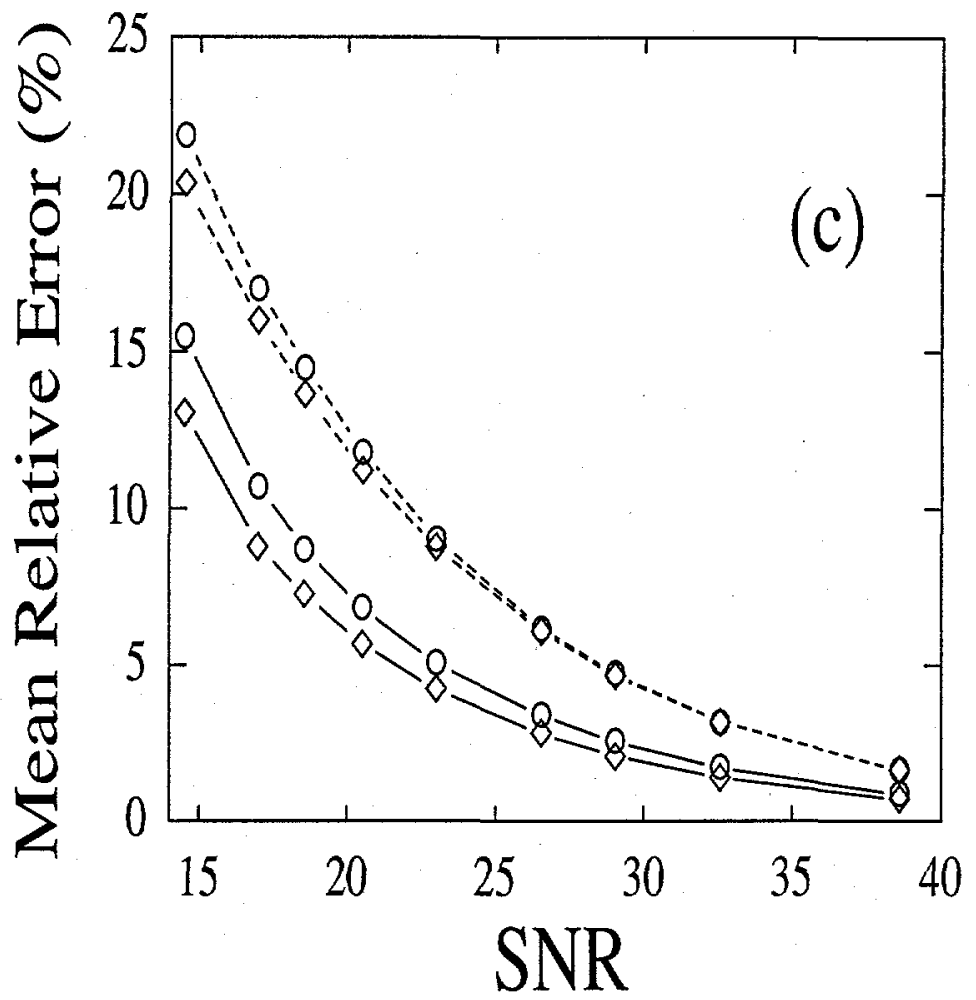

Figure 3.3: Monte Carlo simulations (with 400 noise realizations) on the ${ }^{19} \mathrm{~F}$ pfluorophenol FIDs of Fig. 3.2 as a function of SNR. The curves show the results for ITMPM (solid line) and LPSVD (dashed line) using FID lengths of $N=128$ (o) complex points (sampling from 0 to $2.56 T_{2}$ ) and $N=256(\diamond)$ points (sampling from 0 to $3.84 T_{2}$ ); (a) success rate, (b) MRE of damping factors, and (c) MRE of amplitudes. 
theoretical advantages, is limited if they involve significant computational overheads. ITMPM, however, is significantly faster than methods such as LPSVD, since no high degree polynomial rooting is needed. This is particularly true for general NMR FID lengths of about $1-2 \mathrm{~K}$ points, where the number of floating-point operations (flops) is reduced by roughly an order of magnitude, as measured in their MATLAB implementations. ICMPM can still not compete, however, with the efficiency of the Fast Fourier Transform. The FFT requires computational time proportional to $O\left(N \log _{2} N\right)$, whereas SVD is an $O\left[(N-L) \times L^{2}\right]$ process. Should computational efficiency be of great concern, rapid SVD of a Toeplitz or Hankel matrix can be accomplished by using the Lanczos algorithm[45] and/or explicitly exploiting the Toeplitz or Hankel structure of the data matrix[46]. Moreover, the result of the ITMPM analysis is a table of spectral parameters which either can be used to construct a frequency/time domain spectrum or can be used directly without extra tedious manual operation for peak searching, curve fitting, and intensity integration. With the present processing protocol, results can be obtained in 4.5 minutes of CPU time for 1024 complex data points on a SGI Indigo workstation with $100 \mathrm{MHz}$ R4000 processor and a spectroscopist's intervention is needed only for data transfer (compared to 2 seconds and 40 minutes for FT and LPSVD, respectively).

Example III: ${ }^{1} \mathrm{H}$ Spectrum of Glutamic Acid in $\mathrm{D}_{2} \mathrm{O}$. Classical spectral estimation using Fourier transformation of truncated data sets implicitly assumes that the unobserved data is zero. A blurred spectral estimate is a consequence of such 
an unrealistic assumption. It should be possible to obtain a better estimate by using a priori knowledge to construct a suitable model for the unobserved data. This would eliminate the need for window functions, along with the associated trade-offs of resolution and sensitivity. Moreover, by incorporating lineshape information, parametric methods, such as ITMPM, are able to resolve overlapped signals that cannot be separated in an FT spectrum.

This is illustrated in Fig. 3.4 using the calculated ${ }^{1} \mathrm{H}$ spectrum of glutamic acid in $\mathrm{D}_{2} \mathrm{O}$, whose "true" spectrum is shown in (d). The result of Fourier transforming the FID truncated to 384 complex data points (and zero-filled back to 4096) is shown in Fig. 3.4 (a). The details of the spectrum are obscured and distorted by sinc-wiggles from nearby stronger signals. Fig. 3.4 (b) shows the result of applying an exponential apodizing function to the FID prior to Fourier transformation. This effectively attenuates the sinc-wiggles, at the expense, however, of resolution. In contrast, applying ITMPM to the truncated FID gives a spectrum, Fig. 3.4 (c), in which the sinc-wiggles are suppressed without degrading resolution. This is particularly significant in multidimensional spectroscopy where the indirectly detected dimensions must often be severely truncated to keep the experiment time within reasonable bounds. Clearly ITMPM can effectively reduce the truncation artifacts.

Figure 3.5 plots the singular values obtained during the ITMPM analysis of Fig. 3.4 (c). It is clear from the gradual decrease of the singular values (solid line) that the separation of signal singular values from those associated with noise is not 
straightforward and subjective approaches are likely to be unsatisfactory. By contrast, the minimum of the MDL criterion (dashed line) is relatively well defined.

\subsection{Experimental Applications}

This work has been prompted by our studies of the long-time behavior of free induction decays in solid-state NMR. Apparently high-resolution spectra (more characteristic of solution rather than solid-state NMR) have been obtained by using a sufficiently long delay between simple pulse excitation and data acquisition. This striking phenomenon has been observed in various experiments using static, magicangle spinning, and off-magic angle spinning samples, and has attracted a wide variety of explanations $[3,48,49,50,51,52,5,54,6]$. Extensions of SPEDAS (Single-Pulse Excitation Delayed Acquisition Spectroscopy) to two-dimensional experiments involving ${ }^{1} \mathrm{H}$ COSY and multi-quantum coherence using polycrystalline fumaric acid monoethyl ester (a "rigid" solid in which there is no motional averaging) have also been reported[48, 50].

Investigation of these experiments is hampered by the extremely large phase distortions and severe sensitivity losses (by factors of $10^{-2}-10^{-4}$ ) that result from the long delay between excitation and acquisition. The use of signal averaging to improve the SNR is limited by the long $T_{1}$ spin-lattice relaxation times of solid-state samples. Hence, experimental restrictions on the total acquisition time result in low sensitivity, and potentially poor spectral resolution in multi-dimensional experiments (due 

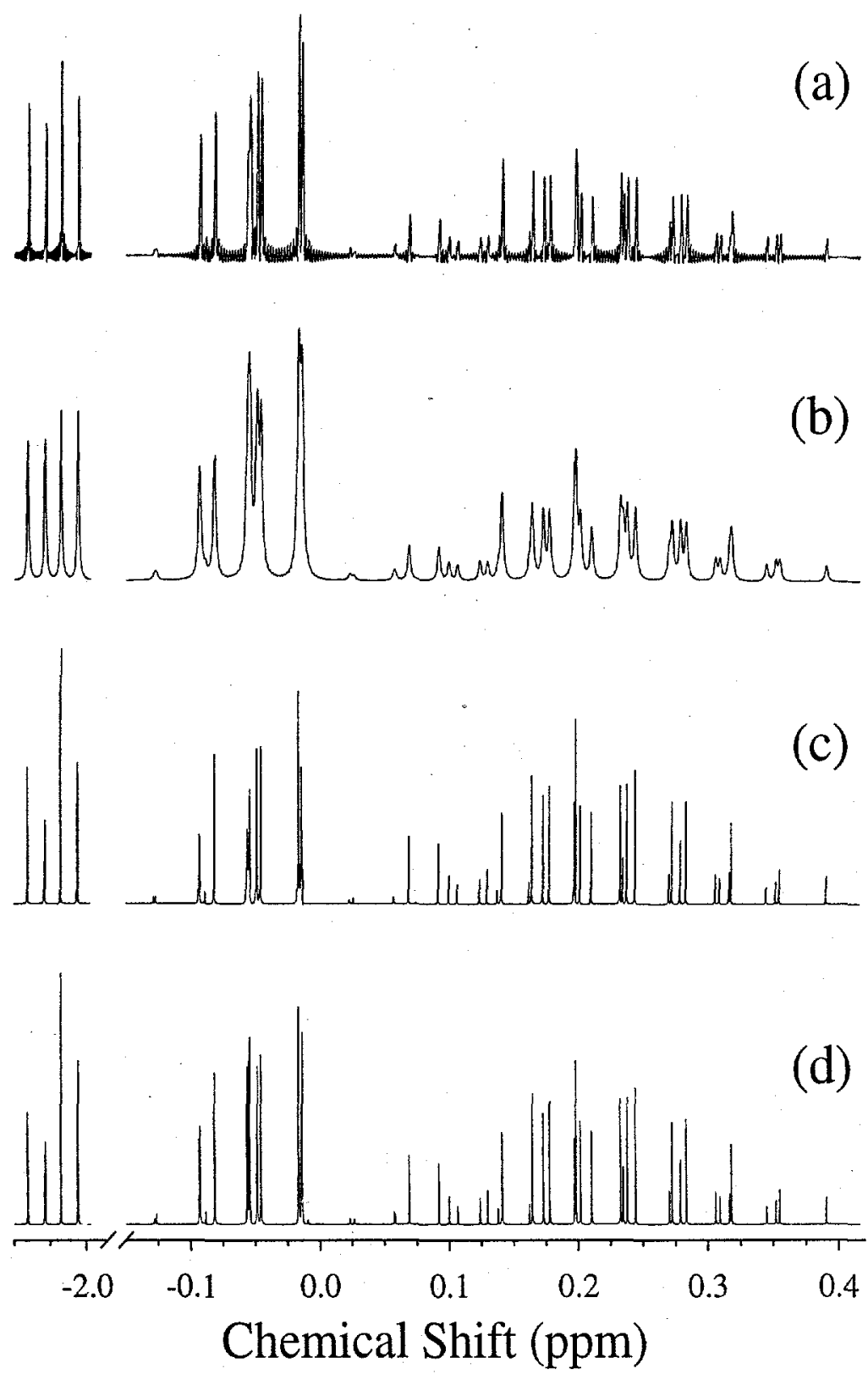

Figure 3.4: Calculated ${ }^{1} \mathrm{H}$ spectra of glutamic acid in $\mathrm{D}_{2} \mathrm{O}$. (a) FT of the FID truncated to 384 data points and zero-filled to 4096 points (SNR $=54 \mathrm{~dB}$ ); (b) FT spectrum of (a) apodized by multiplying the truncated FID by an exponential decay; (c) ITMPM spectrum of (a); (d) FT spectrum of the original FID of 4096 points and $\mathrm{SNR}=\infty$. 


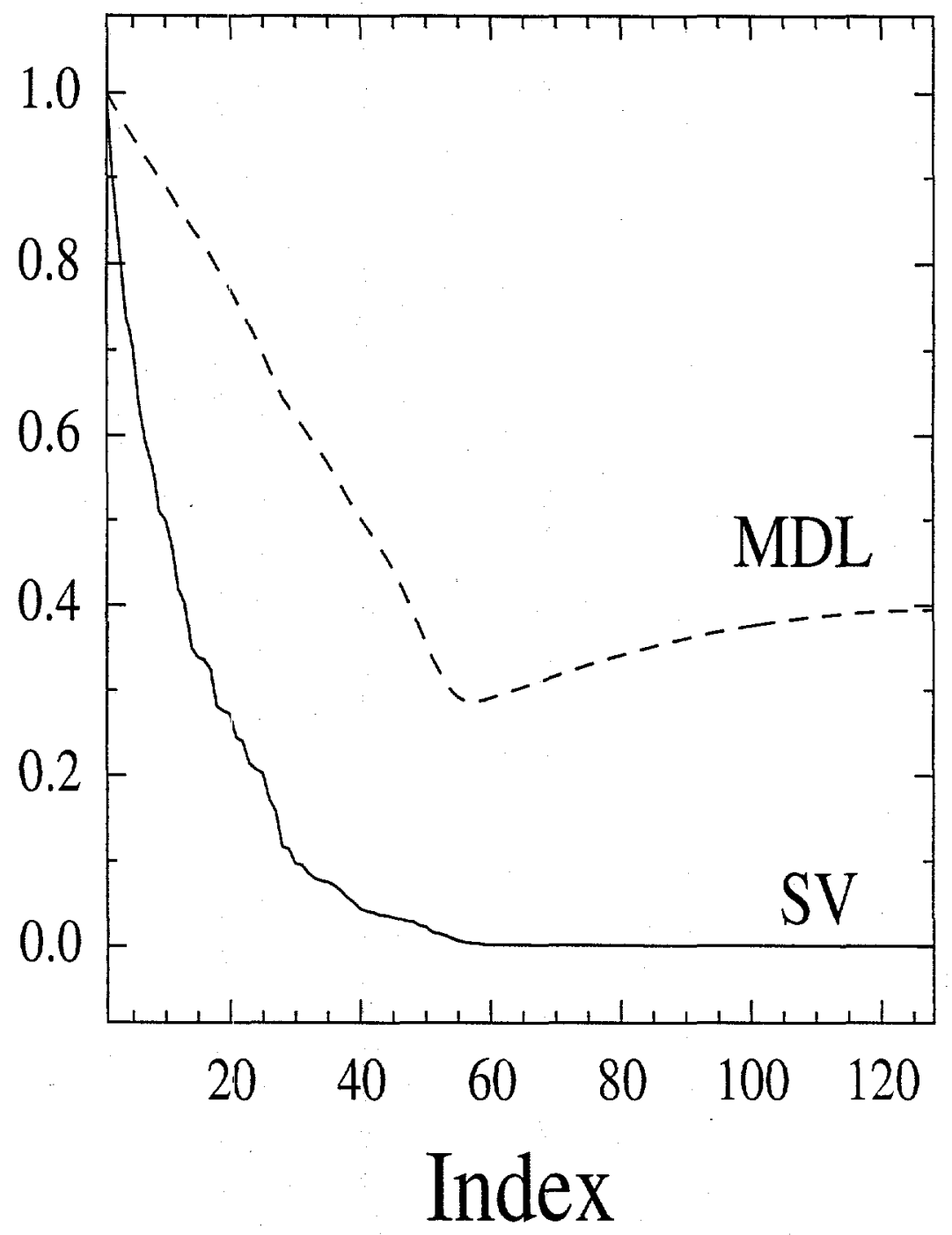

Figure 3.5: The functional value of MDL criterion (dashed line) and the magnitude of the singular values (solid line) (both are normalized) during the ITMPM analysis of Fig. 3.4 (c). The optimal matrix rank determined by MDL is $M=57$. 
to data truncation in the indirectly detected dimensions).

Clearly a more sophisticated spectral estimation method than the Fourier Transform is required to analyze the results of these experiments. Although the nature of the signals is still currently under investigation, our experimental experience suggests that modelling as a sum of exponentials is a reasonable approximation[56]. Figure 3.6 (a) shows the FT spectra using 10,240 and 8,192 scans for static polycrystalline anthracene (upper row) and malonic acid (lower row) respectively. Despite the extensive signal averaging, the features of the spectrum are still difficult to distinguish from the high-level background noise. The corresponding ITMPM spectra are shown in Fig. 3.6 (b). Increasing the number of scans from 10,240 to 198,856 for anthracene and from 8,192 to 117,112 for malonic acid, gives the FT spectra shown in Fig. 3.6 (c). The difference between these "true" spectra and the ITMPM spectra derived from much noisier signals is very small. The ability of ITMPM to extract the signal information from relatively noisy FIDs greatly facilitates the study of the long time behavior of solid-state NMR signals, particularly as the problems of phase correction are eliminated cf. Fig. 3.6 (d).

ITMPM should also be important in other experiments where the FID is truncated or whenever the SNR is low, as demonstrated below. Figure 3.7 (a) presents the FT spectrum of a noisy ${ }^{43} \mathrm{Ca}$ FID from an aqueous solution containing $0.20 \mathrm{M} \mathrm{CaCl}_{2}$ (natural abundance) and $0.10 \mathrm{M}$ sodium EDTA, and acquired with 50,000 scans. Its corresponding ITMPM spectrum is shown in Fig. 3.7 (b). From the chemical com- 


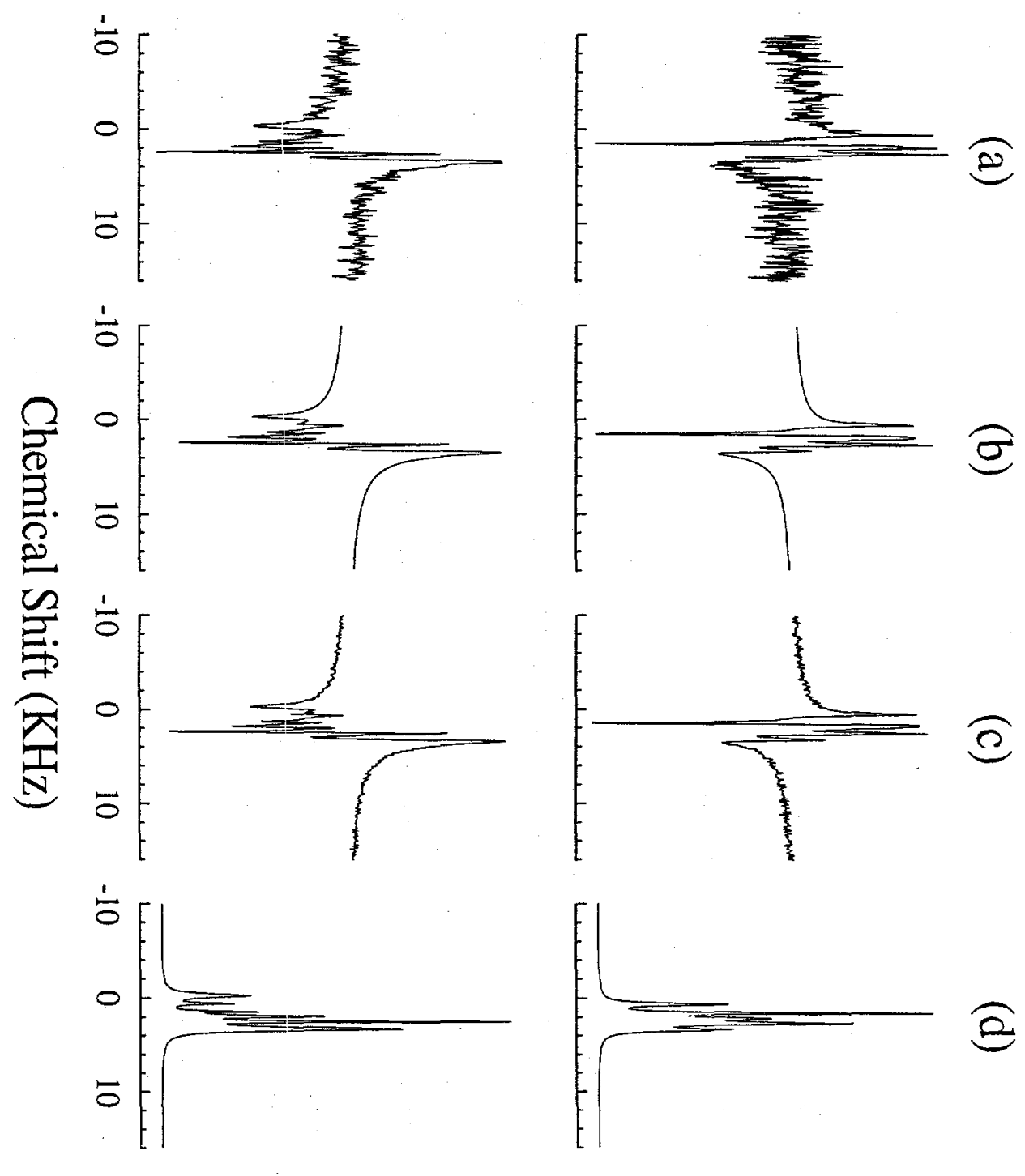

Figure 3.6: Experimental spectra of static polycrystalline anthracene (upper row) and malonic acid (lower row) measured at room temperature on a Bruker AM-400 spectrometer with 231 points and initial delays of $600 \mu \mathrm{s}$ (anthracene) and $856 \mu \mathrm{s}$ (malonic acid). (a) FT spectra of the FIDs obtained by accumulating 10,240 (anthracene) and 8,192 (malonic acid) scans; (b) ITMPM spectra of (a) with the optimal matrix rank, $M=5$ (anthracene) and $M=6$ (malonic acid), determined by the MDL criterion; (c) FT spectra of the FIDs obtained under the same experimental conditions as (a) but accumulating 198,865 (anthracene) and 117,112 (malonic acid) scans. (d) phasecorrected ITMPM spectra, obtained from (b) by setting all the phases to zero. 
position of the solution, it is known that the FID should consist of two exponential components corresponding to free $\mathrm{Ca}^{2+}$ and $\mathrm{Ca}$-EDTA complex. We have also performed another measurement under the same experimental conditions except for an increase of scans from 50,000 to 1,700,000. Its FT spectrum is depicted in Fig. 3.7 (c). and will be used as the "true" spectrum for comparison. Again, the ITMPM spectrum serves as a very close approximation to the "true" spectrum.

\subsection{Conclusion}

An often recurring problem in NMR spectroscopy is how to improve spectral sensitivity and resolution. The Monte-Carlo simulations described above indicate that this combined detection-estimation scheme, ITMPM, is able to simultaneously achieve these objectives. The deficiencies of the applications of the FT to imperfect NMR data can be diminished to a large extent at the expense of reasonable computational complexity. Compared to LPSVD, it has the advantages of greater computational efficiency, higher precision and accuracy of the estimated spectral parameters, and less tendency for spurious estimates at low signal-to-noise ratios. ITMPM is essentially near-optimal over the range of signal-to-noise ratios investigated; the parameter estimates have biases that are close to zero and standard deviations close to their Cramér-Rao Lower Bounds.

For experimental applications, ITMPM considerably facilitates our analysis of the delayed acquisition data, and increases the potential applicability of SPEDAS- 


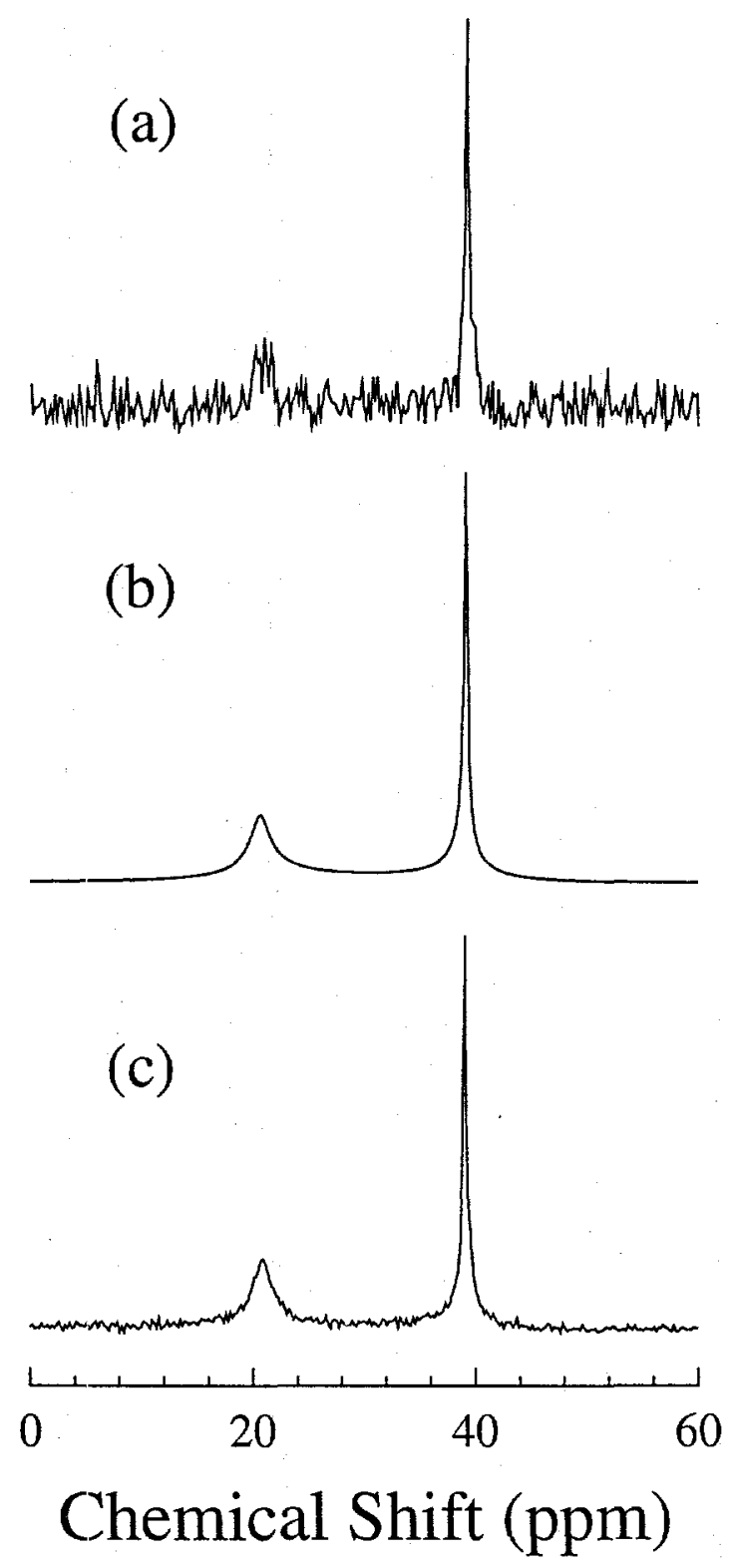

Figure 3.7: Experimental spectra of an aqueous solution containing $0.20 \mathrm{M} \mathrm{CaCl}_{2}$ and $0.10 \mathrm{M}$ sodium EDTA obtained at $300 \mathrm{~K}$ on a Bruker AM-400 spectrometer. (a) FT spectra of the FID obtained by accumulating 50,000 scans; (b) ITMPM spectra of (a) with the optimal matrix rank, $M=2$, determined by the MDL criterion; (c) FT spectra of the FIDs obtained under the same experimental conditions as (a) but accumulating $1,700,000$ scans. 
like experiments to solid-state NMR, by providing phase-corrected spectra with much improved sensitivity and resolution. ITMPM should also be important in other experiments where the FID is truncated or whenever the SNR is low. This is often the case in one- and multi-dimensional NMR studies of low gamma nuclei, in vivo samples, and macromolecules of biological interest. Consequently, ITMPM could become a valuable quantification tool within NMR and other branches of Fourier spectroscopy. 


\section{Bibliography}

[1] J. C. Lindon and A. G. Ferrige, Prog. NMR Spectrosc. 14, 27 (1980).

[2] R. de Beer and D. van Ormondt, in "NMR Basic Principles and Progress" (P. Diehl, E. Fluck, H. Gunther, R. Kosfeld, and J. Seelig, Eds.), Vol. 26, pp. 201, Springer-Verlag, Berlin/Heidelberg, 1992.

[3] R. E. Hoffman and G. C. Levy, Prog. NMR Spectrosc. 23, 211 (1991).

[4] J. J. Led and H. Gesmar, Chem. Rev. 91, 1413 (1991).

[5] R. Roy, B. G. Sumpter, G. A. Pfeffer, S. K. Gray, and D. W. Noid, Physics Reports 205, 109 (1991).

[6] H. Gesmar, J. J. Led, and F. Abildgaard, Prog. NMR Spectrosc. 22, 255 (1990).

[7] D. S. Stephenson, Prog. NMR Spectrosc. 20, 515 (1988).

[8] H. Barkhuijsen; R. de Beer, A. C. Drogendijk, D. van Ormondt, and J. W. C. van der Veen, in 'Proceedings International School of Physics 'Enrico Fermi' on the 
'Physics of NMR Spectroscopy in Biology and Medicine' " (B. Maraviglia, Ed.), pp. 313, Italian Physical Society, 1988.

[9] M. Mehring, "Principles of High Resolution NMR in Solids," 2nd ed., SpringerVerlag, Berlin/New York, 1983.

[10] A. van der Veen, E. F. Deprettere, and A. L. Swindlehurst, Proc. IEEE 81, 1277 (1993).

[11] The Mathworks, Inc., "MATLAB Reference Guide," South Natick, Massachusetts, 1992.

[12] R. Kumaresan and D. W. Tufts, IEEE Trans. Acoust. Speech Signal Process. ASSP-30, 833 (1982).

[13] H. Barkhuijsen, R. de Beer, W. M. M. J. Bovee, and D. van Ormondt, J. Magn. Reson. 61, 465 (1985).

[14] S. M. Kay, "Modern Spectral Estimation," Prentice-Hall, Englewood Cliffs, New Jersey, 1987.

[15] S. L. Marple, "Digital Spectral Analysis with Applications," Prentice-Hall, Englewood Cliffs, New Jersey, 1987.

[16] S. Y. Kung, K. S. Arun, and D. V. Bhaskar Rao, J. Opt. Soc. Am. 73, 1799 (1983). 
[17] H. Barkhuijsen, R. de Beer, and D. van Ormondt, J. Magn. Reson. 73, 553 (1987).

[18] Y. Hua and T. K. Sarkar, IEEE Trans. Signal Process. 39, 892 (1991).

[19] Y. Hua and T. K. Sarkar, IEEE Trans. Acoust. Speech Signal Process. ASSP-38, $814(1990)$.

[20] P. Hodgkinson and P. J. Hore, Adv. Magn. Opt. Reson. 20 (in press).

[21] R. Roy, A. Paulraj, and T. Kailath, IEEE Trans. Acoust. Speech Signal Process. ASSP-34, 1340 (1986).

[22] G. Zhu and A. Bax, J. Magn. Reson. 100, 202 (1992).

[23] J. Tang and J. R. Norris, J. Chem. Phys. 84, 5210 (1986).

[24] J. Tang and J. R. Norris, Chem. Phys. Lett. 131, 252 (1986).

[25] J. Tang. C. P. Lin, M. K. Bowman, and J. R. Norris, J. Magn. Reson. 62, 167 (1985).

[26] Y. K. Lee, R. L. Vold, G. L. Hoatson, Y.-Y. Lin, and A. Pines, J. Magn. Reson. A 112, 112 (1995).

[27] A. C. Kot, D. W. Tufts, and R. J. Vaccaro, IEEE Trans. Signal Process. 41, $3174(1993)$. 
[28] G. H. Golub and C. F. Van Loan, "Matrix Computations," 2nd ed., John Hopkins University Press, Baltimore, 1989.

[29] C. Eckart and G. Young, Psychometrika 1, 211 (1936).

[30] K. Konstantinides and K. Yao, IEEE Trans. Signal Process. 36, 757 (1988).

[31] J. M. Chambers, "Computational Methods for Data Analysis," Wiley, New York, 1977.

[32] J. A. Cadzow, B. Baseghi, and T. Hsu, IEE Proc. pt. F, 130, 202 (1983).

[33] M. S. Bartlett, J. Roy. Stat. Soc. Ser. B, 16, 296 (1954).

[34] D. N. Lawley, Biometrica 43, 128 (1956).

[35] I. Dologlou and G. Carayannis, IEEE Trans. Acoust. Speech Signal Process. ASSP-39, 1681 (1991).

[36] M. Shinnar and S. M. Eleff, J. Magn. Reson. 76, 200 (1988).

[37] M. Wax and T. Kailath, IEEE Trans. Acoust. Speech Signal Process. ASSP-33, $387(1985)$.

[38] H. Akaike, IEEE Trans. Automat. Contr. AC-19, 716 (1974).

[39] G. Schwartz, Ann. Stat. 6, 461 (1978).

[40] J. Rissanen, Automatica 14, 465 (1978). 
[41] Y.-M. Wang, H. Lee, and D. V. Apte, Int. J. Imag. Sys. Tech. 4, 201 (1992).

[42] H. Ouibrahim, IEEE Trans. Acoust. Speech Signal Process. ASSP-37, 133 (1989).

[43] B. D. Rao, IEEE Trans. Acoust. Speech Signal Process. ASSP-36, 1026 (1988).

[44] P. Koehl, C. Ling, and J. F. Lefèvre, J. Magn. Reson. A 109, 32 (1994).

[45] G. L. Millhauser, A. A. Carter, D. J. Schneider, J. H. Freed, and R. E. Oswald, J. Magn. Reson. 82, 150 (1989).

[46] W. W. F. Pijnappel, A. van den Boogaart, R. de Beer, and D. van Ormondt, J. Magn. Reson. 97, 122 (1992).

[47] S. Ding and C. A. McDowell, J. Magn. Reson. A 111, 212 (1994).

[48] S. Ding and C. A. McDowell, J. Magn. Reson. A 115, 141 (1995).

[49] S. Ding and C. A. McDowell, J. Magn. Reson. A 117, 171 (1995).

[50] S. Ding and C. A. McDowell, J. Magn. Reson. A 120, 261 (1996).

[51] S. Ding and C. A. McDowell, Chem. Phys. Lett. 255, 151 (1996).

[52] S. Ding and C. A. McDowell, Chem. Phys. Lett. 259, 538 (1996).

[53] B. C. Gerstein, J. Z. Hu, J. Zhou, C. Ye, M. Solum, R. Pugmire, and D. M. Grant, Solid State NMR 6, 63 (1996). 
[54] J. Z. Hu, J. Zhou, F. Deng, H. Feng, N. Yang, L. Li, and C. Ye, Solid State NMR 6, 85 (1996).

[55] B. M. Fung, T. Dollase, M. L. Magnuson, and T.-H. Tong, J. Magn. Reson. A 123, $56(1996)$.

[56] M. Engelsberg and I. J. Lowe, Phys. Rev. B 10, 822 (1974).

[57] S. A. Smith, T. O. Levante, B. H. Meier, and R. R. Ernst, J. Magn. Reson. A 106, 75 (1994). 


\section{Part II}

\section{Spin Dynamics in Quantum}

Dissipative Systems 



\section{Chapter 4}

\section{Solitonic Spin Dynamics}

\subsection{Abstract}

The existence of the solitonic modes in the NMR transverse relaxation of dipolecoupled many-spin systems is experimentally confirmed and theoretically modeled by a spin-boson Hamiltonian to describe the influence of the fluctuating dipolar localfields upon the quantum coherence in connection with the macroscopic observable. Such striking phenomena, totally unexpected within current NMR theoretical framework, originate from the nonlinearity triggered by the joint action of the lattice fluctuations and the reaction field: the spins fluctuate and polarize the lattice which reacts back on the spins. 


\subsection{Introduction}

For abundant spins in solids, particularly those with high gyromagnetic ratios such as ${ }^{1} \mathrm{H}$ and ${ }^{19} \mathrm{~F}$, the homonuclear dipolar coupling $\mathcal{H}_{d}$ is the dominant internal interaction. For $N$ equivalent spin dipoles under a large static longitudinal Zeeman field, the effective Hamiltonian $\mathcal{H}$ in the rotating frame precessing with the Larmor frequency $\omega_{\circ}$ can be approximated by its secular part $\mathcal{H}_{d}^{\circ}$ (in angular-frequency unit)

$$
\begin{aligned}
\mathcal{H} & =\sum_{i=0}^{N-1} \sum_{i<j} \mathcal{H}_{d, i j}^{\circ}, \\
\mathcal{H}_{d, i j}^{\circ} & =D_{i j}\left[2 I_{z i} I_{z j}-\left(I_{x i} I_{x j}+I_{y i} I_{y j}\right)\right] \\
D_{i j} & =\frac{\gamma^{2} \hbar}{2} \frac{1-3 \cos ^{2} \theta_{i j}}{r_{i j}^{3}}
\end{aligned}
$$

where $\gamma$ is the gyromagnetic ratio, $\boldsymbol{I}$ is the spin angular-momentum operator, $r$ is the internuclear distance, and $\theta$ is the angle between the internuclear vector and the Zeeman field [2]. The flip-flop interaction $\left(I_{+i} I_{-j}+I_{-i} I_{+j}\right)$, embedded in the $I_{x i} I_{x j}$ or $I_{y i} I_{y j}$ term, excites the mutual spin flips, disturbing the phase of an individual spin without energy loss in the entire system. Such dephasing process, as reflected in the measured free induction decay, is a subject that has been of great theoretical and experimental interest since the birth of magnetic resonance. The understanding is crucial to the interpretation of experimental results and the development of new methodologies in NMR and related techniques, let alone its fundamental importance in quantum and statistical mechanics. Since the optical pseudo-dipolar coupling 
also share similar Hamiltonian structure, any resulting comprehension may be easily generalized to its counterpart in optical spectroscopies.

The many-body character and the absence of a small parameter in the Hamiltonian preclude any exact and general analysis covering the whole duration of the phase relaxation process in various free induction decay experiments. Nevertheless, numerous approaches, mainly based on moment expansions and stochastic local-field models[1], have been proposed and can indeed quantitatively or qualitatively describe the shorttime dephasing behavior: Gaussian decays in rigid solids subject to a quasi-static distribution of the local-fields, and exponential decays in liquids and solids with a strong exchange interaction in which rapid local-field fluctuations are present. Recent experimental work by Ding and McDowell, however, suggests the possible existence of anomalous long-time behavior [3]. Without resorting to demanding decoupling techniques in the spin and/or spatial spaces, apparently high-resolution solid-state NMR spectra can be obtained simply by using a sufficiently long delay between simple pulse excitation and data acquisition to filter out the initial fast-decaying part before performing Fourier transformation. This striking phenomenon has been demonstrated in various experiments using static, magic-angle spinning, and off magic-angle spinning samples [4], and has attracted a wide variety of explanations. The original authors claim that it is an intrinic property of the bulk rigid lattice and can be explained by a memory effect with no clear physical foundation. Other authors, however, suggest that it may result from the motional narrowing effects associated with a small frac- 
tion of thermally activated mobile species present in the heterogeneous structures of the rigid lattice due to lattice defects, surface disorder, or moisture adsorption[5]. It has also been indicated that there may exist specific crystalline orientations at which the total dipolar coupling vanishes, or that the homogeneously broadened spectral peak may consist of a superposition of numerous transitions with different linewidths [6]. In this chapter, we ask the following questions: does this anomalous long-time behavior really exist, and if there is any, what is its physical origin?

\subsection{Experimental Verification}

To reach an experimental confirmation, static NMR measurements at liquidhelium temperature were carried out first. The low temperature ensures that the samples, static polycrystalline glycine and alanine, are "rigid" solids without significant thermal motion. To eliminate the effects of moisture, $4 \mathrm{~mm}$ quartz tubes containing the sample were attached to a vacuum line $\left(\leq 10^{-5} \mathrm{mmHg}\right)$; first dried at $50^{\circ} \mathrm{C}$ for 2 days and then sealed. Experiments were performed on a home-built spectrometer with a home-built transmission-line probe assembly operating at a ${ }^{1} \mathrm{H}$ frequency of $\omega_{0} / 2 \pi=178.025 \mathrm{MHz}$. Figures 4.1 and 4.2 show the short-time and long-time behaviors of the ${ }^{1} \mathrm{H}$ free induction decays.

According to contemporary NMR theory, for typical ${ }^{1} \mathrm{H}$ coupling networks in solids, the transverse magnetization is completely dephased within tens of microseconds and therefore results in the broad peak in spectrum (a). The much sharper 

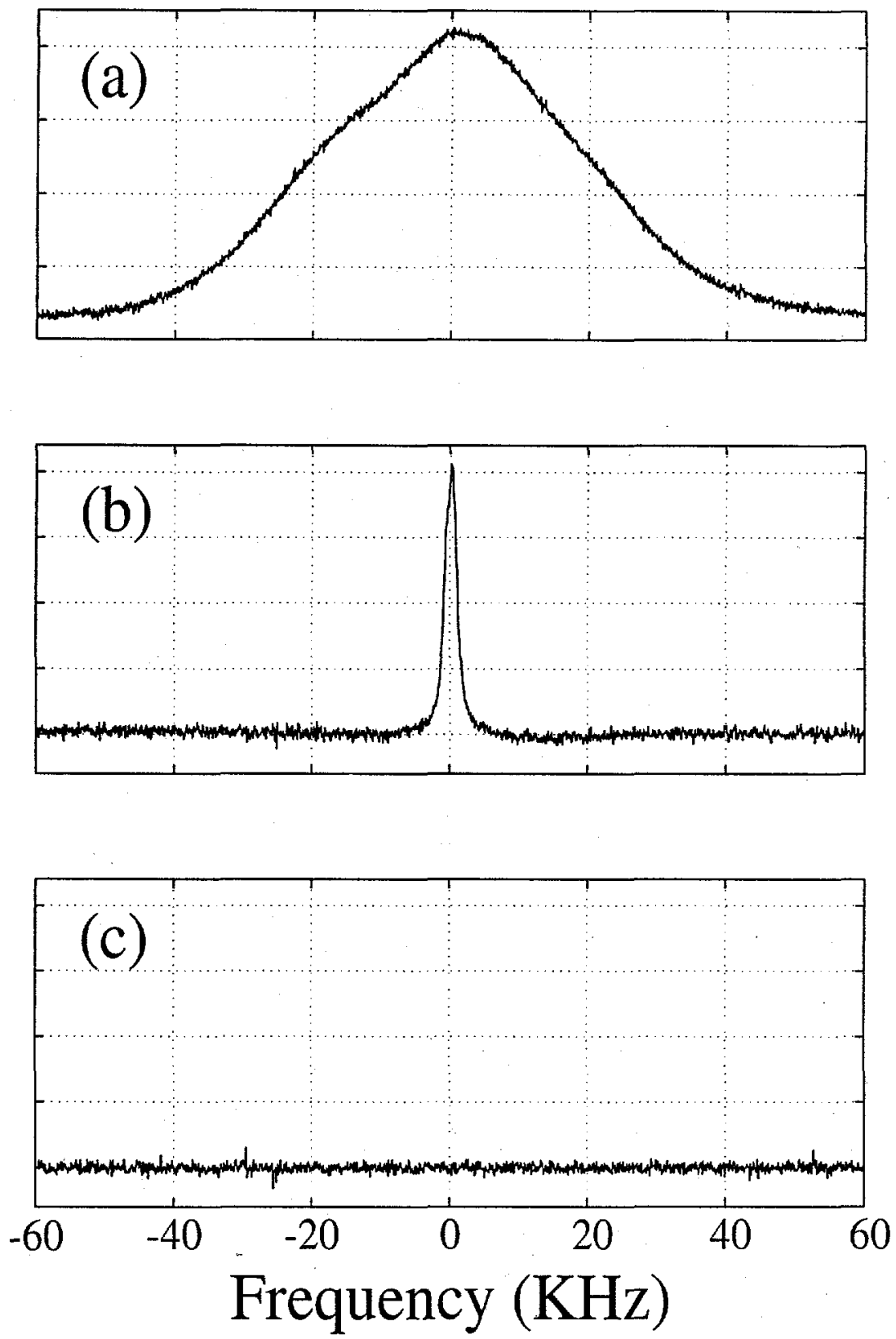

Figure 4.1: ${ }^{1} \mathrm{H}$ NMR spectra of static polycrystalline glycine (MCB Chemicals, $99.5 \%$ ), measured at $4 \mathrm{~K}$ with single $\pi / 2$ pulse excitation and phase cycling. (a) normal spectrum obtained by accumulating 32 scans; (b) frequency spectra of the free induction decay acquired with 2000 scans and an extra $100 \mu$ s acquisition delay; (c) the background spectrum under the same experimental conditions as (b). 

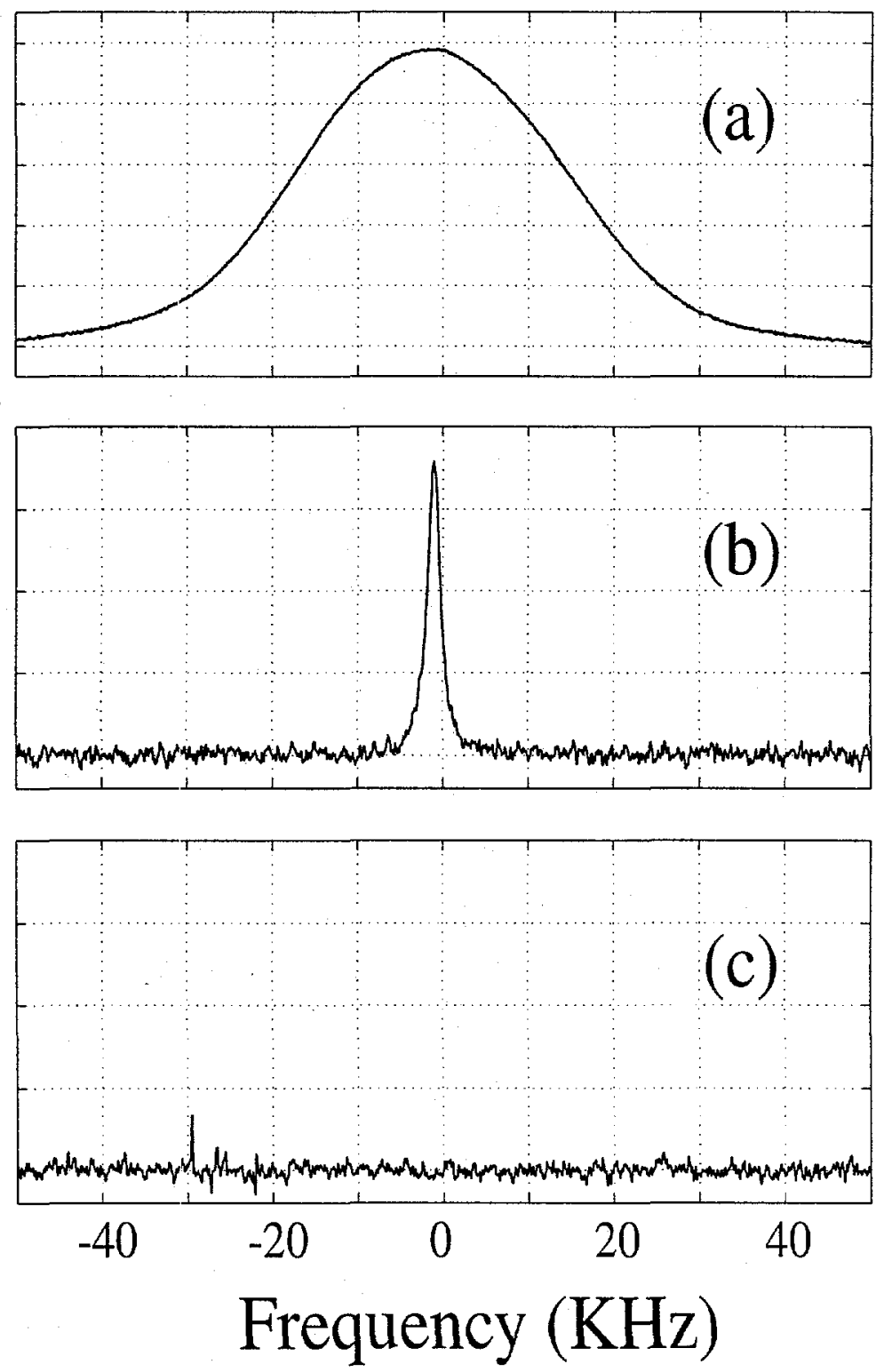

Figure 4.2: ${ }^{1} \mathrm{H}$ NMR spectra of static polycrystalline alanine (Sigma Chemical Co., $99.5 \%$ ) measured at $4 \mathrm{~K}$ with single $\pi / 2$ pulse excitation and phase cycling. (a) normal spectrum obtained by accumulating 160 scans; (b) frequency spectra of the free induction decay acquired with 200 scans and an extra $200 \mu$ s acquisition delay; (c) the background spectrum under the same experimental conditions as (b). 
peak in spectrum (b) clearly shows that the magnetization in fact persists much longer than the usually defined phase relaxation time $\mathrm{T}_{2}$. By comparing with the background spectrum (c), the possibilities of experimental or instrumental artifacts may be mostly eliminated.

\subsection{Spin-Boson Hamiltonian}

The spectra shown in Figs. 4.1 and 4.2 correspond to the Fourier transform of the macroscopic observable $\left\langle\sum_{i=0}^{N-1} I_{x i}(t)\right\rangle$. In the case of equivalent spins, one gets $\left\langle\sum_{i=0}^{N-1} I_{x i}(t)\right\rangle \approx N\left\langle I_{x 0}(t)\right\rangle$, where the subscript "o" labels the tagged spin. Rewrite Eq. (4.1) in the one-spin picture,

$$
\begin{aligned}
\mathcal{H} & =-\omega_{z} I_{z 0}-\omega_{x} I_{x 0}-\omega_{y} I_{y 0}+\mathcal{H}_{L} \\
\omega_{z} & \equiv-2 \sum_{j=1}^{N-1} D_{0 j} I_{z j} \\
\omega_{x} & \equiv \sum_{j=1}^{N-1} D_{0 j} I_{x j} \\
\omega_{y} & \equiv \sum_{j=1}^{N-1} D_{0 j} I_{y j} \\
\mathcal{H}_{L} & \equiv \sum_{i=1}^{N-1} \sum_{i<j} \mathcal{H}_{d, i j}^{\circ}
\end{aligned}
$$

where $\omega_{x}, \omega_{y}$, and $\omega_{z}$ are the dipolar local-fields acting on the tagged spin. Following the convention of NMR, the terms "spin" and "lattice" (" $L ")$ will be used to represent 
the primary and all the secondary degrees of freedom, respectively. The initial density operator in the laboratory frame prepared by a $\pi / 2$ pulse along $-y$ axis can be approximated as

$$
\rho(0) \propto \sinh \left(\beta \omega_{\circ} / 2\right)|+\rangle_{x}\left\langle+\left.\right|_{x} \exp \left[-\beta\left(\omega_{x}+U^{\dagger} \mathcal{H}_{L} U\right)\right]\right.
$$

where $U=\exp \left(1 \pi / 2 \sum_{j=1}^{N-1} I_{y j}\right), \beta$ is the inverse lattice temperature and $| \pm\rangle_{x}$ is defined by $I_{x}| \pm\rangle_{x}= \pm \frac{1}{2}| \pm\rangle_{x}$. A general scheme to describe the evolution of $\left\langle I_{x 0}(t)\right\rangle$ is to first solve the set of coupled Heisenberg equations associated with the Hamiltonian and then take ensemble average over the initial density operator $\rho(0)$. For our dipolecoupled many-spin systems, it still represents a formidable task. This is the starting point of various different approaches and approximations.

First, assume that the lattice can be modeled as a collection of generalized harmonic oscillators. A reservoir consisting of harmonic oscillators is rather general and often provides a suitable mean-field Langevin description of a realistic environment[7]. For example, even the coupling to a Fermionic bath (as in our case) may be well described by the Bosonic environment considered here [6]. Second, in many-spin systems, the autocorrelation time in the longitudinal local-field $\omega_{z}$ is characterized by $(3-4) \mathrm{T}_{2}$, which is much slower than that in the transverse local-field $\omega_{x}$. Therefore, during this time period, $\omega_{z}$ can be temporarily assumed to be a constant. Finally, for mathematical tractability, the fluctuating local-field along the $y$-axis is ignored. 
Based on these assumptions, Eqs. (4.4) and (4.9) become

$$
\begin{gathered}
\mathcal{H}=-\omega_{z} I_{z}-\lambda \omega_{x} I_{z}+\sum_{j} \nu_{j} b_{j}^{\dagger} b_{j} \\
\rho(0) \propto|+\rangle_{x}\left\langle+\left.\right|_{x} \exp \left[-\beta\left(\alpha \omega_{x}+\sum_{j} \nu_{j} b_{j}^{\dagger} b_{j}\right)\right]\right.
\end{gathered}
$$

where $\omega_{x}=\sum_{j} \Gamma_{j}\left(b_{j}+b_{j}^{\dagger}\right)$, and $b_{j}$ and $b_{j}^{\dagger}$ are respectively the annihilation and creation operators for the $j$ th boson of frequency $\nu_{j}$ with coupling constant $\Gamma_{j}$. The parameter $\lambda$ characterizes the intensity of the spin-lattice coupling and also serves as a perturbation variable in later analysis. We introduce the parameter $\alpha$ to study the dependence of the long-time dynamics on the initial density operator, especially on the magnitude of the dipolar order. Equation (4.10) is the famous spin-boson model [5], which is now adopted to describe in the rotating frame the influence of the dissipative dipolar local-fields on the dynamics of a tagged spin within a fully Hamiltonian picture. To test the validity of the above assumptions, we have performed molecular-dynamics simulations for classical spins governed by secular dipolar Hamiltonian. The results (Chapter 7) also exhibit similar long-time dynamics, as discuss ?d below.

\subsection{Reaction Field}

Denote the unperturbed motion of the the transverse local-field with $\omega_{x}^{(0)}(t)$,

$$
\omega_{x}^{(0)}(t)=\sum_{j} \Gamma_{j}\left(b_{j} \mathrm{e}^{-i \nu_{j} t}+b_{j}^{\dagger} \mathrm{e}^{i \nu_{j} t}\right)
$$


This term describes the free fluctuations of the lattice and is usually referred as the $d i$ rect field. The initial statistical distribution of the oscillators is canonical with respect to the shifted harmonic potential of Eq. (4.11), therefore $\left\langle\omega_{x}^{(0)}(t)\right\rangle \neq 0$. Following the same lines as Vitali et al.[10], it is then convenient to adopt the following polaronic transformation

$$
\begin{aligned}
\tilde{b}_{j} & \equiv b_{j}+\frac{\lambda \Gamma_{j}}{\nu_{j}} \alpha, \\
\tilde{\omega}_{x} & \equiv \sum_{j} \Gamma_{j}\left(\tilde{b}_{j}+\tilde{b}_{j}^{\dagger}\right)
\end{aligned}
$$

so that $\tilde{\omega}_{x}^{(0)}(t)$ is now a Gaussian stochastic operator with vanishing mean value. Note that $I_{x}, I_{y}$, and $I_{z}$ are invariant under such transformation. By solving the Heisenberg equation in the new reference frame for $\tilde{\omega}_{x}(t)$, one finds

$$
\tilde{\omega}_{x}(t)=\tilde{\omega}_{x}^{(0)}(t)+\tilde{\omega}_{x}^{(R)}(t)
$$

where $\tilde{\omega}_{x}^{(0)}(t)$ and $\tilde{\omega}_{x}^{(R)}(t)$ are defined in Eq. (4.12) and

$$
\tilde{\omega}_{x}^{(R)}(t)=2 \lambda \int_{0}^{t} d \tau\left\{\sum_{j} \Gamma_{j}^{2} \sin \left[\nu_{j}(t-\tau)\right]\right\}\left[I_{x}(\tau)+\alpha\right]
$$

The second term $\tilde{\omega}_{x}^{(R)}(t)$ in Eq. (4.15) is usually referred as the reaction field since it takes into account the effect that the spin fluctuates and polarizes the lattice which 
reacts back on the spin. The reaction field $\tilde{\omega}_{x}^{(R)}(t)$, which depends on the state of the tagged spin, makes the spin evolution nonlinear and nonstationary.

By assuming $\tilde{\omega}_{x}^{(R)}(t) \approx\left\langle\tilde{\omega}_{x}^{(R)}(t)\right\rangle$, ignoring the quantum fluctuations (i.e., the imaginary part) of the direct-field correlation function $\left\langle\tilde{\omega}_{x}^{(0)}(t) \tilde{\omega}_{x}^{(0)}(0)\right\rangle$, and carrying out a second-order perturbation in $\lambda$, one reaches the following integro-differential equation (Chapter 5)

$$
\begin{aligned}
\frac{d}{d t}\left\langle I_{x}(t)\right\rangle= & -\omega_{z} \int_{0}^{t} d \tau \mathrm{e}^{-\int_{\tau}^{t} d \tau^{\prime} \gamma_{1}\left(\tau^{\prime}\right)}\left[\omega_{z}+\gamma_{2}(\tau)\right]\left\langle I_{x}(\tau)\right\rangle \cos \left\{\int_{\tau}^{t} d \tau^{\prime} 2 \alpha\left[B\left(\tau^{\prime}\right)-\Delta\right]\right\} \\
& +\frac{1}{2} \omega_{z} \int_{0}^{t} d \tau \mathrm{e}^{-\int_{\tau}^{t} d \tau^{\prime} \gamma_{1}\left(\tau^{\prime}\right)} A(\tau) \sin \left\{\int_{\tau}^{t} d \tau^{\prime} 2 \alpha\left[B\left(\tau^{\prime}\right)-\Delta\right]\right\}
\end{aligned}
$$

where $\Delta, \gamma_{1}(t), \gamma_{2}(t), A(t)$, and $B(t)$ are defined by

$$
\begin{aligned}
\Delta & \equiv \lambda^{2} \sum_{j} \frac{\Gamma_{j}^{2}}{\nu_{j}} \\
\gamma_{1}(t) & \equiv \lambda^{2} \int_{0}^{t} d \tau\left\langle\tilde{\omega}_{x}^{(0)}(0) \tilde{\omega}_{x}^{(0)}(-\tau)\right\rangle \cos \left(\omega_{z} \tau\right) \\
\gamma_{2}(t) & \equiv \lambda^{2} \int_{0}^{t} d \tau\left\langle\tilde{\omega}_{x}^{(0)}(0) \tilde{\omega}_{x}^{(0)}(-\tau)\right\rangle \sin \left(\omega_{z} \tau\right) \\
A(t) & \equiv \lambda^{2} \int_{0}^{t} d \tau\left[\sum_{j} \Gamma_{j}^{2} \sin \left(\nu_{j} \tau\right)\right] \sin \left(\omega_{z} \tau\right) \\
B(t) & \equiv \lambda^{2} \int_{0}^{t} d \tau \sum_{j} \Gamma_{j}^{2} \sin \left(\nu_{j} \tau\right) .
\end{aligned}
$$

When time $t=\infty, \gamma_{1}(\infty)$ is the spectral component of the transverse local-field at frequency $\omega_{z}$ and $A(\infty)$ has the physical meaning of the equilibrium Boltzman magnetization under a constant $\omega_{z}$ field. 
To render it possible to compare the above theoretical prediction with experiments, the distribution of the bosons must be specified. Assume that the number of oscillators is infinite with frequencies distributed over a continuum, so that complete information about the effect of the lattice is encapsulated in the single "spectral density" $J(\nu)$ defined as

$$
J(\nu) \equiv \lambda^{2} \sum_{j} \Gamma_{j}^{2} \delta\left(\nu-\nu_{j}\right)
$$

where $\delta$ is the Dirac delta function. For our spin system, the lattice may be empirically described by the "constrained Ohmic" spectral density

$$
\begin{gathered}
J(\nu)=\frac{\lambda^{2} c}{\nu_{c}^{2}} \nu \exp \left(-\nu / \nu_{c}\right), \\
\int_{0}^{\infty} J(\nu) d \nu=\lambda^{2} c
\end{gathered}
$$

where $\nu_{c}$ is the cut-off frequency and $\lambda^{2} c$ is determined by the lattice structure and dipolar coupling strength.

\subsection{Solitonic Modes}

The transverse magnetization $\left\langle I_{x}(t)\right\rangle$ and reaction field $\left\langle\tilde{\omega}_{x}^{(R)}(t)\right\rangle$ can now be numerically solved from Eqs. (4.18) and (4.17) respectively, as depicted in Figs. 4.3 and 4.4 for various values of $\omega_{z}$ and $\lambda$. Further averaging over the Gaussian probability 
distribution of $\omega_{z}$ for various sites in a bulk sample produces spectra in qualitative agreement with the experiments shown in Figs. 4.1and 4.2.

The reaction field, weak in the short-time region, becomes increasingly important upon the increase of time. Consider the case of small tipping angle $(\alpha=-1 / 2)$. In the short-time limit, $\left\langle I_{x}(t)\right\rangle \approx 1 / 2$ and $\left\langle\tilde{\omega}_{x}^{(R)}(t)\right\rangle \approx 0$, while in the long-time limit $\left\langle I_{x 0}(t)\right\rangle \approx 0$ and $\left\langle\tilde{\omega}_{x}^{(R)}(t)\right\rangle \approx \lambda^{2} c / \nu_{c}$. In other words, the spin feels in the $x$-axis an increasing biased field $\tilde{\omega}_{x}^{(R)}(t)$ with an asymptotic strength of $\lambda^{2} c / \nu_{c}$, in addition to a Gaussian stochastic field $\tilde{\omega}_{x}^{(0)}$. After an initial transit time, the spin precesses around a tilted axis with an $x$ component depending on the spin state itself. Adopting the language of soliton theory, the spin is temporarily localized in a state with $\left\langle I_{x}(\infty)\right\rangle \neq 0$ due to the similar mechanism proposed by Davydov for the localization and transport of chemically produced vibrational energy in protein: the exciton-phonon coupling leads to vibrations about new equilibrium positions[11]. The fluctuations of the Gaussian field $\tilde{\omega}_{x}^{(0)}$ and the longitudinal local-field $\omega_{z}$ will eventually provoke the destruction of this localized state with an extended time scale which is an exponential function of the dipolar coupling strength $\lambda$, in a way similar to the thermal instability of the Davydov soliton at physiological temperature[12].

\subsection{Conclusion}

In summary, the role of the reaction field is the missing key property of current NMR relaxation theories. The lattice fluctuations are not independent of the spin 

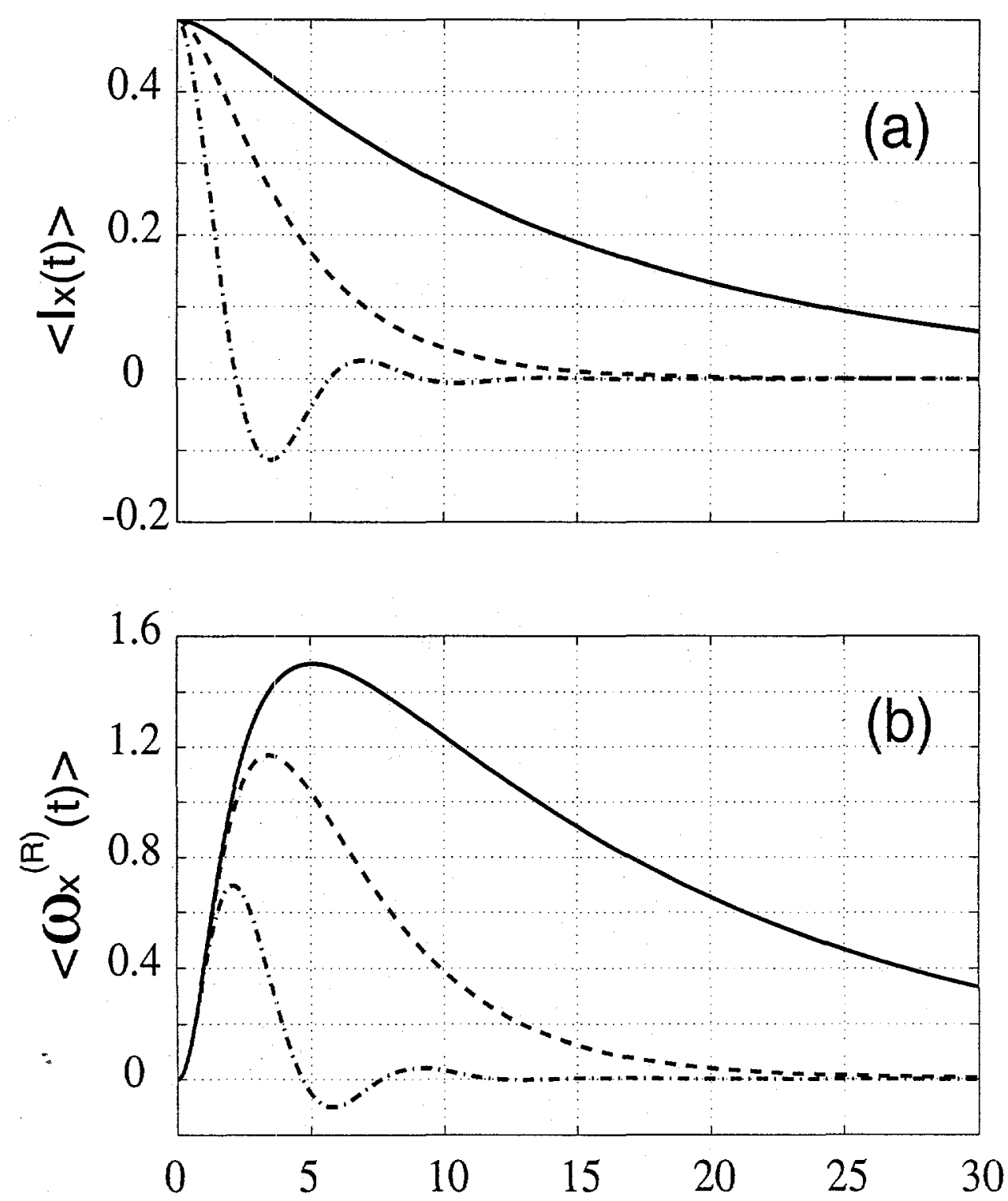

Figure 4.3: The evolution of (a) the transverse magnetization $\left\langle I_{x}(t)\right\rangle$ and (b) the reaction field $\left\langle\tilde{\omega}_{x}^{(R)}(t)\right\rangle$, obtained by numerically solving Eqs. (4.18) and (4.17), respectively. The longitudinal local field $\omega_{z}=0.25$ (solid line), 0.5 (dash line), and 1.0 (dash-dot line). Other parameters used are: $\alpha=0,\left\langle I_{x}(0)\right\rangle=0.5$, cut-off frequency $\nu_{c}=0.5$, and $\lambda=c=1$. 

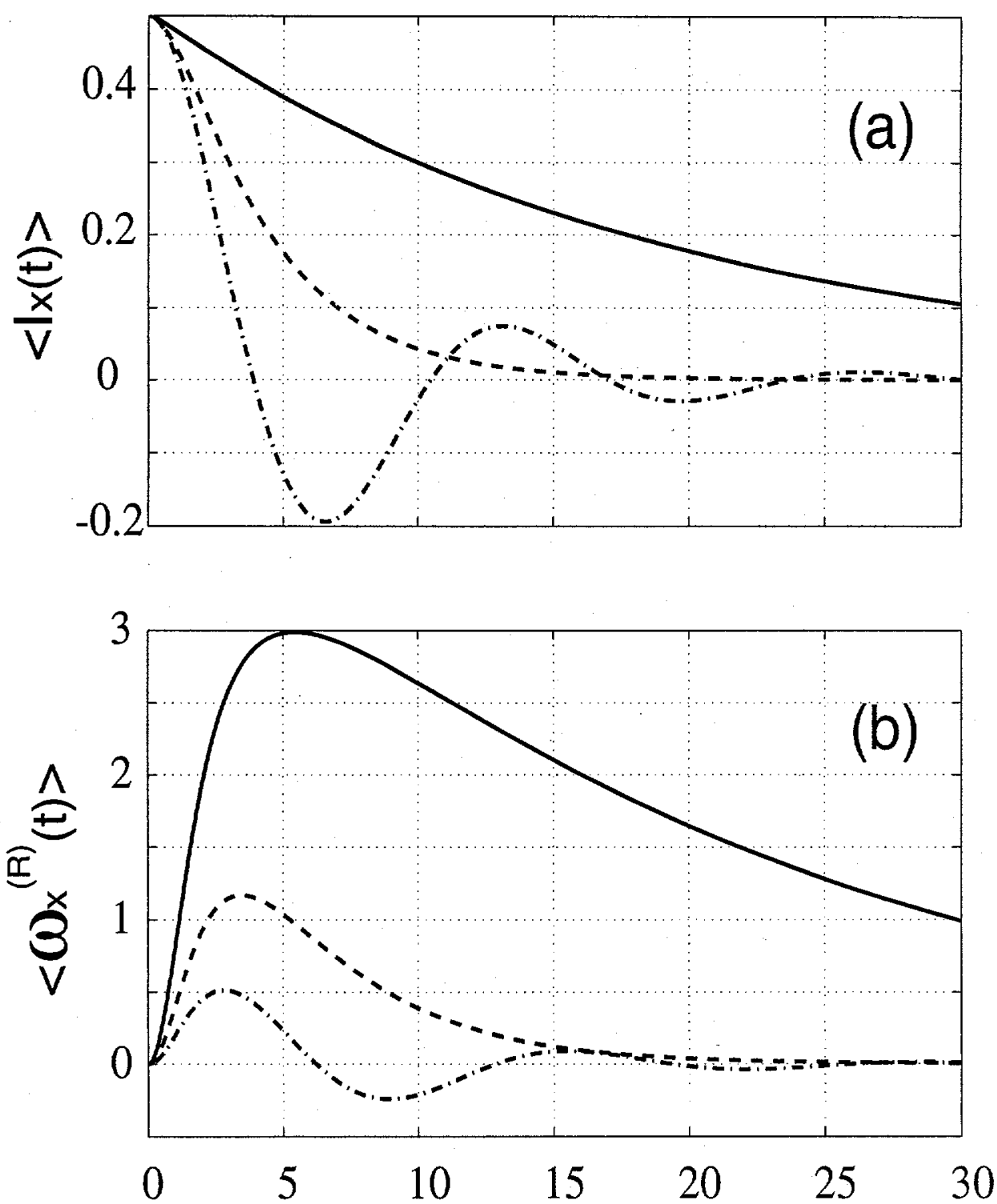

Figure 4.4: The evolution of (a) the transverse magnetization $\left\langle I_{x}(t)\right\rangle$ and (b) the reaction field $\left\langle\tilde{\omega}_{x}^{(R)}(t)\right\rangle$, obtained by numerically solving Eqs. (4.18) and (4.17), respectively. The spin-lattice coupling parameter $\lambda=2$ (solid line), 1 (dash line), and 0.5 (dash-dot line). Other parameters used are: $\omega_{z}=0.5, \alpha=0,\left\langle I_{x}(0)\right\rangle=0.5$, cut-off frequency $\nu_{c}=0.5$, and $c=1$. 
states, and the crucial problem is precisely how to take this effect into account. In this regard, our simple perturbation treatment based on the spin-boson Hamiltonian supplements the prototype approaches with proper inclusion of the reaction field in addition to the direct field in describing the dynamics of the spins coupled to a dissipative lattice. It therefore can provide a qualitative explanation on the physical mechanism of the experimentally confirmed solitonic modes in phase relaxation. When the condition of weak spin-lattice coupling (i.e., small $\lambda$ ) or weal dopolar order (i.e., small $\alpha$ ), tacitly implied by normal NMR experimental conditions, is dismissed, the interplay among the lattice fluctuations and the reaction field, and hence the resulting nonlinear polaronic/solitonic dynamics, can be expected to be even more pronounced. 


\section{Bibliography}

[1] A. Abragam, Principles of Nuclear Magnetism (Clarendon, Oxford, 1961).

[2] See, e.g., E. T. Cheng and J. D. Memory, Phys. Rev. B 6, 1714 (1972); R. Kubo and N. Hashitsume, Prog. Theor. Phys. Suppl. 46, 210 (1970); P. W. Anderson and P. R. Weiss, Rev. Mod. Phys. 25, 269 (1953).

[3] S. Ding and C. A. McDowell, J. Magn. Reson. A 111, 212 (1994).

[4] S. Ding and C. A. McDowell, J. Magn. Reson. A 115, 141 (1995); ibid. 117, 171 (1995); ibid. 120, 261 (1996); S. Ding and C. A. McDowell, Chem. Phys. Lett. 255, 151 (1996); ibid. 259, 538 (1996).

[5] B. C. Gerstein, J. Z. Hu, J. Zhou, C. Ye, M. Solum, R. Pugmire, and D. M. Grant, Solid State NMR 6, 63 (1996); J. Z. Hu, J. Zhou, F. Deng, H. Feng, N. Yang, L. Li, and C. Ye, Solid State NMR 6, 85 (1996).

[6] B. M. Fung, T. Dollase, M. L. Magnuson, and T.-H. Tong, J. Magn. Reson. A 123, 56 (1996). 
[7] M. Moshinsky and Y. F. Smirnov, The Harmonic Oscillator in Modern Physics (Harwood Academic Publisher, Amsterdam, 1996).

[8] U. Weiss, Quantum Dissipative Systems (World Scientific, New Jersey, 1993).

[9] A. J. Leggett, S. Chakravarty, A. T. Dorsey, M. P. A. Fisher, A. Garg and W. Zwerger, Rev. Mod. Phys. 59, 1 (1987).

[10] D. Vitali, L. Bonci, R. Mannella, and P. Grigolini, Phys. Rev. A 45, 2285 (1992).

[11] A. S. Davydov, Biology and Quantum Mechanics (Pergamon Press, New York, 1982).

[12] For a recent review, see A. Scott, Phys. Rep. 217, 1 (1992). 


\section{Chapter 5}

\section{Spin-Boson Hamiltonian}

\subsection{Abstract}

We approach the problem of spin dynamics in quantum dissipative systems starting from a microscopic and quantum-mechanical Hamiltonian of a two-level system coupled to a collection of independent bosonic elementary excitations. Based on the polaronic transform and a second-order perturbation treatment, an analytical solution governing the evolution of $\left\langle I_{x}(t)\right\rangle$ is derived, suggesting that, upon decrease of the longitudinal local field, $\left\langle I_{x}(t)\right\rangle$ should exhibit a critical transition to an overdamped self-trapped regime. The dynamics of this process is expected to be influenced by the joint action of the bath fluctuations and of the nonlinearity stemming from the reaction field. The model is also extended to two-dimensional spin-boson Hamiltonian. 


\subsection{Introduction}

The relaxation of spin system is a subject that has been of great theoretical and experimental interest for a long time. Such understanding is crucial to the interpretation of experimental results and development of new methodology in NMR and ESR. The analysis of spin relaxation is usually based on the following three approaches. One is the quantum mechanical density matrix approach pioneered by Bloch, Redfield, and others [1]. Another scheme is developed by Kubo, Anderson and Weiss and is based on classical multiplicative stochastic equations of motion for the spin components $[2,3]$. The third method is the semiclassical stochastic Hamiltonian formulation developed by Fox [4]. A pervasive feature of some of these approaches is that the description of the dynamics of spin relaxation is based either on untested approximations or on semi-phenomenological assumptions. For example, in the early versions of the fully quantum mechanical treatment, it is assumed at the outset that the density operator can at all times be expressed as the product of a system and a bath density operator, the bath being canonically distributed at all times. Another common feature of these models is the ubiquitous appearance of linear relaxation laws for the average spin components, i.e. of Bloch or Bloch-like equations.

The spin-boson model provides a simple approach to describe the influence of the dissipative environment on the dynamics of a quantum system within a fully 
Hamiltonian picture [5]

$$
\mathcal{H}=-\omega_{z} I_{z}-\lambda \omega_{x} I_{x}+\sum_{j} \nu_{j} b_{j}^{\dagger} b_{j}
$$

where

$$
\begin{gathered}
\omega_{x} \equiv \sum_{j} \omega_{x j}, \\
\omega_{x j} \equiv \Gamma_{j}\left(b_{j}+b_{j}^{\dagger}\right),
\end{gathered}
$$

and $b_{j}$ and $b_{j}^{\dagger}$ are respectively the annihilation and creation operators for the $j$ th boson of frequency $\nu_{j}$ with coupling constant $\Gamma_{j}$. The parameter $\lambda$ characterizes the intensity of the spin-lattice coupling and also serves as a perturbation variable in later analysis. Because of its simplicity, this model Hamiltonian has been studied in many different areas, to name a few, condensed-matter physics[6], chemical physics[7, 8, 9], and the foundation of quantum mechanics[10]. This is a strongly nonisotropic model with fluctuations only along one axis, while in a typical NMR problem the environment provides fluctuating fields that are in all directions. Instead of quantitatively reproducing the NMR spin relaxation process, this work aim at understanding the nonlinear dynamics stemming from the reaction field within a model that has the essential aspects of spin relaxation. The discussion is limited to the following initial 
density operator (Chapter 4)

$$
\rho(0)=|+\rangle_{x}\left\langle+\left.\right|_{x} \exp \left[-\beta\left(\lambda \alpha \omega_{x}+\sum_{j} \nu_{j} b_{j}^{\dagger} b_{j}\right)\right]\right.
$$

where $\alpha$ is a parameter used to study the dependence of spin dynamics on the initial density operator, especially on the magnitude of the dipolar order.

\subsection{Polaronic 'Transformation}

The Heisenberg equation for an arbitrary quantum-mechanical operator $O(t)$ is given by

$$
\frac{d}{d t} O(t)=-1[O(t), \mathcal{H}]
$$

Therefore, the set of Heisenberg equations derived from the spin-boson Hamiltonian of Eq. (5.1) reads[11]

$$
\begin{aligned}
\frac{d}{d t} I_{x}(t) & =\omega_{z} I_{y}(t), \\
\frac{d}{d t} I_{y}(t) & =-\omega_{z} I_{x}(t)+\lambda \omega_{x}(t) I_{z}(t), \\
\frac{d}{d t} I_{z}(t) & =-\lambda \omega_{x}(t) I_{y}(t), \\
\frac{d^{2}}{d t^{2}} \omega_{x j}(t)+\nu_{j}^{2} \omega_{x j}(t) & =2 \lambda \nu_{j} \Gamma_{j}^{2} I_{x}(t),
\end{aligned}
$$


Here we have used the following relations:

$$
\begin{gathered}
{\left[b_{j}-b_{j}^{\dagger}, b_{j}+b_{j}^{\dagger}\right]=2,} \\
{\left[b_{j}-b_{j}^{\dagger}, b_{j}^{\dagger} b_{j}\right]=b_{j}+b_{j}^{\dagger} .}
\end{gathered}
$$

The fluctuating field along the $x$ axis is proportional to the coordinate $\omega_{x}(t)$. If we assume these fluctuations to be fast and their mean value to vanish, we are immediately led by Eqs. (5.6)-(5.9) to a zeroth-order picture, obtained by setting $\omega_{x}(t)=0$. The resulting spin dynamics is a precession around the $z$ axis with precessional frequency $\omega_{z}$

A more refined picture must take the role of the fluctuation into account, especially in the case when it is not much faster than the spin dynamics. Because of the initial condition of Eq. (5.4), the unperturbed motion of the collective coordinate $\omega_{x}(t)$, which will be denoted with $\omega_{x}^{(0)}(t)$, is such that $\left\langle\omega_{x}^{(0)}(t)\right\rangle \neq 0$. Then it is convenient to shift the reference frame so as to make the mean value of the new unperturbed coordinate, $\tilde{\omega}_{x}(t)$, vanish. This condition is fulfilled by adopting the polaronic transform[12, 13, 14]:

$$
\begin{aligned}
\tilde{b}_{j} & \equiv b_{j}+\frac{\lambda \Gamma_{j}}{\nu_{j}} \alpha, \\
\tilde{\omega}_{x j} & \equiv \Gamma_{j}\left(\tilde{b}_{j}+\tilde{b}_{j}^{\dagger}\right), \\
\tilde{\omega}_{x} & \equiv \sum_{j} \tilde{\omega}_{x j} .
\end{aligned}
$$


The initial condition of Eq. (5.4) expressed in terms of the new operators is

$$
\rho(0)=|+\rangle_{x}\left\langle+\left.\right|_{x} \exp \left[-\beta \sum_{j} \nu_{j} \tilde{b}_{j}^{\dagger} \tilde{b}_{j}\right]\right.
$$

As a consequence of adopting the new reference framework, the collective and unperturbed coordinate $\tilde{\omega}_{x}^{(0)}(t)$ is a Gaussian stochastic operator, with vanishing mean value that is completely defined by the two-time correlation function

$$
\begin{gathered}
\left\langle\tilde{x}^{(0)}\left(t_{1}\right) \cdots \tilde{x}^{(0)}\left(t_{2 n+1}\right)\right\rangle=0 \\
\left\langle\tilde{x}^{(0)}\left(t_{1}\right) \cdots \tilde{x}^{(0)}\left(t_{2 n}\right)\right\rangle=\sum_{\text {pair }}\left\langle\tilde{x}^{(0)}\left(t_{i_{1}}\right) \tilde{x}^{(0)}\left(t_{i_{2}}\right)\right\rangle \cdots\left\langle\tilde{x}^{(0)}\left(t_{i_{2 n-1}}\right) \tilde{x}^{(0)}\left(t_{i_{2 n}}\right)\right\rangle
\end{gathered}
$$

with order preserved [15].

In the new reference system, the set of Heisenberg equations of Eqs. (5.6)-(5.9) becomes

$$
\begin{aligned}
\frac{d}{d t} I_{x}(t) & =\omega_{z} I_{y}(t) \\
\frac{d}{d t} I_{y}(t) & =-\omega_{z} I_{x}(t)-2 \Delta \alpha I_{z}(t)+\lambda \tilde{\omega}_{x}(t) I_{z}(t) \\
\frac{d}{d t} I_{z}(t) & =2 \Delta \alpha I_{y}(t)-\lambda \tilde{\omega}_{x}(t) I_{y}(t) \\
\frac{d^{2}}{d t^{2}} \tilde{\omega}_{x j}(t)+\nu_{j}^{2} \tilde{\omega}_{x j}(t) & =2 \lambda \nu_{j} \Gamma_{j}^{2}\left[I_{x}(t)+\alpha\right]
\end{aligned}
$$


where

$$
\Delta \equiv \lambda^{2} \sum_{j} \frac{\Gamma_{j}^{2}}{\nu_{j}} .
$$

Solving Eq. (5.21) one obtains

$$
\begin{aligned}
\tilde{\omega}_{x}(t) & =\tilde{\omega}_{x}^{(0)}(t)+\tilde{\omega}_{x}^{(R)}(t) \\
\omega_{x}^{(0)}(t) & =\sum_{j} \Gamma_{j}\left(b_{j} \mathrm{e}^{-1 \nu_{j} t}+b_{j}^{\dagger} \mathrm{e}^{1 \nu_{j} t}\right) \\
\tilde{\omega}_{x}^{(R)}(t) & =2 \lambda \int_{0}^{t} d \tau\left\{\sum_{j} \Gamma_{j}^{2} \sin \left[\nu_{j}(t-\tau)\right]\right\}\left[I_{x}(\tau)+\alpha\right]
\end{aligned}
$$

The first term $\tilde{\omega}_{x}^{(0)}(t)$ describes the free fluctuations of the lattice in the absence of the interaction with the spin-1/2 system and is usually referred as the direct field, while the second term $\tilde{\omega}_{x}^{(R)}(t)$ is usually referred as the reaction field since it takes into account the effect that the spin fluctuates and polarizes the lattice which reacts back on the spin. The reaction field $\tilde{\omega}_{x}^{(R)}(t)$, which depends on the state of the spin-1/2 system, makes the spin dynamics nonlinear and nonstationary.

Replacing Eq. (5.23) into the set of Heisenberg equations Eqs. (5.18)-(5.21), one 
gets

$$
\begin{aligned}
\frac{d}{d t} I_{x}(t)= & \omega_{z} I_{y}(t) \\
\frac{d}{d t} I_{y}(t)= & -\omega_{z} I_{x}(t)-2 \Delta \alpha I_{z}(t)+\lambda\left[\tilde{\omega}_{x}^{(0)}(t) I_{z}(t)\right]_{S} \\
& +2 \lambda^{2} \int_{0}^{t} d \tau\left\{\sum_{j} \Gamma_{j}^{2} \sin \left[\nu_{j}(t-\tau)\right]\right\}\left[\left(I_{x}(\tau)+\alpha\right) I_{z}(t)\right]_{S} \\
\frac{d}{d t} I_{z}(t)= & 2 \Delta \alpha I_{y}(t)-\lambda\left[\tilde{\omega}_{x}^{(0)}(t) I_{y}(t)\right]_{S} \\
& -2 \lambda^{2} \int_{0}^{t} d \tau\left\{\sum_{j} \Gamma_{j}^{2} \sin \left[\nu_{j}(t-\tau)\right]\right\}\left[\left(I_{x}(\tau)+\alpha\right) I_{y}(t)\right]_{S}
\end{aligned}
$$

where

$$
[a b]_{S} \equiv \frac{1}{2}(a b+b a)
$$

for two arbitrary operators $a$ and $b$. Equations (5.26)-(5.28) are formally exact. When $\lambda=0$ the system is characterized by harmonic oscillations with frequency $\omega_{z}$. They then describe how this ideal behavior is perturbed by the interaction between the spin of interest and its bath of oscillators.

\subsection{Second-Order Perturbation}

To gain a deeper understanding and make the physics more transparent, it is convenient to approximate Eqs. (5.26)-(5.28) in some relevant limit conditions. In the following, we carry out a perturbation expansion at second order in the parameter 
$\lambda$. All the results of this section are valid without making any particular assumption on the spectral density and so they will be expressed in general in terms of the couplings $\Gamma_{j}$. To be consistent with the second-order perturbation treatment, the two-time operators $\left[I_{x}(\tau) I_{z}(t)\right]_{S}$ and $\left[I_{x}(\tau) I_{y}(t)\right]_{S}$, in the last term on the right hand side (rhs) of the second and third equations, must be evaluated at the zeroth order. From Eqs. (5.26)-(5.28), one gets the following zeroth-order approximations

$$
\begin{aligned}
\frac{d}{d t} I_{x}(t) & =\omega_{z} I_{y}(t), \\
\frac{d}{d t} I_{y}(t) & =-\omega_{z} I_{x}(t), \\
\frac{d}{d t} I_{z}(t) & =0
\end{aligned}
$$

Solving the set of differential equations, one obtains the zeroth-order solutions

$$
\begin{aligned}
& I_{x}(t)=I_{x}(0) \cos \omega_{z} t+I_{y}(0) \sin \omega_{z} t \\
& I_{y}(t)=I_{y}(0) \cos \omega_{z} t-I_{x}(0) \sin \omega_{z} t \\
& I_{z}(t)=I_{z}(0)
\end{aligned}
$$

Therefore, the two-time operators, when evaluated at the zeroth order, become

$$
\begin{gathered}
{\left[I_{x}(\tau) I_{z}(t)\right]_{S}=0} \\
{\left[I_{x}(\tau) I_{y}(t)\right]_{S}=\frac{1}{4} \sin \left[\omega_{z}(\tau-t)\right] .}
\end{gathered}
$$


Here we have used the following properties

$$
\begin{aligned}
& {\left[I_{x}(t) I_{y}(t)\right]_{S}=\left[I_{y}(t) I_{z}(t)\right]_{S}=\left[I_{z}(t) I_{x}(t)\right]_{S}=0} \\
& {\left[I_{x}(t) I_{x}(t)\right]_{S}=\left[I_{y}(t) I_{y}(t)\right]_{S}=\left[I_{z}(t) I_{z}(t)\right]_{S}=\frac{1}{4}}
\end{aligned}
$$

Still focusing on Eqs. (5.26)-(5.28), we note that $I_{z}(t)$, appearing in the third term on the right-hand side (rhs) of the second equation, and $I_{y}(t)$, in the second term on the rhs of the third equation, should be evaluated at the first order in $\lambda$. The first order approximation to Eqs. (5.26)-(5.28) reads

$$
\begin{aligned}
\frac{d}{d t} I_{x}(t) & =\omega_{z} I_{y}(t), \\
\frac{d}{d t} I_{y}(t) & =-\omega_{z} I_{x}(t)+\lambda\left[\tilde{\omega}_{x}^{(0)}(t) I_{z}(t)\right]_{S}, \\
\frac{d}{d t} I_{z}(t) & =-\lambda\left[\tilde{\omega}_{x}^{(0)}(t) I_{y}(t)\right]_{S} .
\end{aligned}
$$

Solving the set of differential equations, one obtains the first-order solutions

$$
\begin{aligned}
I_{x}(t)= & I_{x}(0) \cos \left(\omega_{z} t\right)+I_{y}(0) \sin \left(\omega_{z} t\right) \\
& +\lambda \int_{0}^{t} d \tau \sin \left[\omega_{z}(t-\tau)\right]\left[\tilde{\omega}_{x}^{(0)}(\tau) I_{z}(\tau)\right]_{S}, \\
I_{y}(t)= & I_{y}(0) \cos \left(\omega_{z} t\right)-I_{x}(0) \sin \left(\omega_{z} t\right) \\
& +\lambda \int_{0}^{t} d \tau \cos \left[\omega_{z}(t-\tau)\right]\left[\tilde{\omega}_{x}^{(0)}(\tau) I_{z}(\tau)\right]_{S}, \\
I_{z}(t)= & I_{z}(0)-\lambda \int_{0}^{t} d \tau\left[\tilde{\omega}_{x}^{(0)}(\tau) I_{y}(\tau)\right]_{S} .
\end{aligned}
$$


Insert the zeroth-order and first-order solutions into Eqs. (5.26)-(5.28) and then take ensemble average:

$$
\begin{aligned}
\frac{d}{d t}\left\langle I_{x}(t)\right\rangle= & \omega_{z}\left\langle I_{y}(t)\right\rangle \\
\frac{d}{d t}\left\langle I_{y}(t)\right\rangle= & -\omega_{z}\left\langle I_{x}(t)\right\rangle-2 \Delta \alpha\left\langle I_{z}(t)\right\rangle-\lambda^{2} \int_{0}^{t} d \tau\left\langle\tilde{\omega}_{x}^{(0)}(0) \tilde{\omega}_{x}^{(0)}(-\tau)\right\rangle\left\langle I_{y}(t-\tau)\right\rangle \\
& +2 \lambda^{2} \alpha \int_{0}^{t} d \tau \sum_{j} \Gamma_{j}^{2} \sin \left[\nu_{j}(t-\tau)\right]\left\langle I_{z}(t)\right\rangle \\
\frac{d}{d t}\left\langle I_{z}(t)\right\rangle= & 2 \Delta \alpha\left\langle I_{y}(t)\right\rangle-\lambda^{2} \int_{0}^{t} d \tau\left\langle\tilde{\omega}_{x}^{(0)}(0) \tilde{\omega}_{x}^{(0)}(-\tau)\right\rangle\left\langle I_{z}(t-\tau)\right\rangle \cos \left(\omega_{z} \tau\right) \\
& -2 \lambda^{2} \int_{0}^{t} d \tau \sum_{j} \Gamma_{j}^{2} \sin \left[\nu_{j}(t-\tau)\right] \frac{1}{4} \sin \left[\omega_{z}(\tau-t)\right] \\
& -2 \lambda^{2} \alpha \int_{0}^{t} d \tau \sum_{j} \Gamma_{j}^{2} \sin \left[\nu_{j}(t-\tau)\right]\left\langle I_{y}(t)\right\rangle
\end{aligned}
$$

Here we have invoked the key assumption that $\tilde{\omega}_{x}^{(R)}(t) \approx\left\langle\tilde{\omega}_{x}^{(R)}(t)\right\rangle$. Its physical reasons will be discussed below.

\subsection{Mean-Field Approximation}

Form a general point of view, the motion of the two-level system can be seen as a superposition of three processes with distinct time scales. First, there is a slow and systematic process. As demonstrated in Chapter 4, in the weak coupling limit (i.e., small $\lambda$ or large $\omega_{z}$ ), this is a damped oscillatory motion with frequency $\approx \omega_{z}$. In the strong-coupling limit, this motion becomes much slower. Thus we can say that the time scale of the systematic motion of the spin is comparable to $\omega_{z}^{-1}$ or else 
larger. The second process characterizing the dynamics of the spin system is due to the interaction with the bath of oscillators. This interaction, in addition to heavily affecting the free oscillations with frequency $\omega_{z}$, also makes the system fluctuate with approximately the same time scale as that of the bath. The third and fastest process concerns the merely quantum-mechanical fluctuations of the system.

The bath coordinate $\tilde{\omega}_{x}$ certainly is affected by the first process, since this is much slower than its own dynamics. In principle, the coordinate $\tilde{\omega}_{x}$ can also be influenced by the second process, with precisely its own time scale. As to the quantum fluctuation, on the contrary, it must be imagined as being an infinitely fast process stemming from the uncertainty principle itself of quantum mechanics, and the dynamics of $\tilde{\omega}_{x}$ can be safely imagined as being independent of them. This would lead us to the following sound assumption:

$$
\tilde{\omega}_{x}(t) \approx\left\langle\tilde{\omega}_{x}(t)\right\rangle_{S}
$$

where we adopt the following definition:

$$
\left\langle\tilde{\omega}_{x}(t)\right\rangle_{S} \equiv \operatorname{Tr}_{S}\left[\tilde{\omega}_{x}(t) \rho_{S}(0)\right]
$$

and $\rho_{S}(0)=|+\rangle_{x}\left\langle+\left.\right|_{x}\right.$ is the initial density operator of the spin-1/2 dipole. This 
means that the following assumption should be adopted:

$$
\tilde{\omega}_{x}^{(R)}(t) \approx\left\langle\tilde{\omega}_{x}^{(R)}(t)\right\rangle_{S} .
$$

Equation (5.51) is supposed to be the best possible approximation because it only sets equal to zero the time scale of the quantum fluctuations. Unfortunately, this scheme involves the moments of $I_{x}(t)$ at all orders. To get a closed equation for $\left\langle I_{x}(t)\right\rangle$ alone, we must have recourse to the less accurate approximation

$$
\tilde{\omega}_{x}^{(R)}(t) \approx\left\langle\tilde{\omega}_{x}^{(R)}(t)\right\rangle
$$

which is sort of mean-field approximation. This is equivalent to saying that the dynamics of the coordinate $\tilde{\omega}_{x}(t)$ is affected only by the systematic part of the spin motion, but not by the thermal and quantum fluctuations around it. This is not a completely correct physical assumption, since the thermal fluctuations of the spin system have the same time scale as that of the bath, which therefore should be affected by them. However, we believe that keeping the mean value of the reaction field is certainly a beeter approximation than neglecting it completely.

The time evolution of $I_{y}(t-\tau)$ and $I_{z}(t-\tau)$ from $t-\tau$ up to $t$, within their respective time-convoluted expressions, must be evaluated at the zeroth order in $\lambda$ to 
have a consistent second-order perturbation treatment in $\lambda$. This means

$$
\begin{aligned}
& \left\langle I_{y}(t-\tau)\right\rangle \approx\left\langle I_{y}(t)\right\rangle \cos \left(\omega_{z} \tau\right)+\left\langle I_{x}(t)\right\rangle \sin \left(\omega_{z} \tau\right) \\
& \left\langle I_{z}(t-\tau)\right\rangle \approx\left\langle I_{z}(t)\right\rangle .
\end{aligned}
$$

By adopting the above approximations, one finally reaches

$$
\begin{aligned}
\frac{d}{d t}\left\langle I_{x}(t)\right\rangle= & \omega_{z}\left\langle I_{y}(t)\right\rangle \\
\frac{d}{d t}\left\langle I_{y}(t)\right\rangle= & -\omega_{z}\left\langle I_{x}(t)\right\rangle-2 \Delta \alpha\left\langle I_{z}(t)\right\rangle-\gamma_{1}(t)\left\langle I_{y}(t)\right\rangle \\
& -\gamma_{2}(t)\left\langle I_{x}(t)\right\rangle+2 \alpha B(t)\left\langle I_{z}(t)\right\rangle \\
\frac{d}{d t}\left\langle I_{z}(t)\right\rangle= & 2 \Delta \alpha\left\langle I_{y}(t)\right\rangle-\gamma_{1}(t)\left\langle I_{z}(t)\right\rangle+\frac{1}{2} A(t)-2 \alpha B(t)\left\langle I_{y}(t)\right\rangle .
\end{aligned}
$$

where $\gamma_{1}(t), \gamma_{2}(t), A(t)$, and $B(t)$ are defined as

$$
\begin{aligned}
\gamma_{1}(t) & \equiv \lambda^{2} \int_{0}^{t} d \tau\left\langle\tilde{\omega}_{x}^{(0)}(0) \tilde{\omega}_{x}^{(0)}(-\tau)\right\rangle \cos \left(\omega_{z} \tau\right) \\
\gamma_{2}(t) & \equiv \lambda^{2} \int_{0}^{t} d \tau\left\langle\tilde{\omega}_{x}^{(0)}(0) \tilde{\omega}_{x}^{(0)}(-\tau)\right\rangle \sin \left(\omega_{z} \tau\right) \\
A(t) & \equiv \lambda^{2} \int_{0}^{t} d \tau\left[\sum_{j} \Gamma_{j}^{2} \sin \left(\nu_{j} \tau\right)\right] \sin \left(\omega_{z} \tau\right) \\
& =-2 \lambda^{2} \int_{0}^{t} d \tau\left\{\sum_{j} \Gamma_{j}^{2} \sin \left[\nu_{j}(t-\tau)\right]\right\} \frac{1}{4} \sin \left[\omega_{z}(\tau-t)\right] \\
B(t) & \equiv \lambda^{2} \int_{0}^{t} d \tau \sum_{j} \Gamma_{j}^{2} \sin \left(\nu_{j} \tau\right)=2 \lambda^{2} \int_{0}^{t} d \tau \sum_{j} \Gamma_{j}^{2} \sin \left[\nu_{j}(t-\tau)\right]
\end{aligned}
$$

The final step is to describe the evolution of $\left\langle I_{x}(t)\right\rangle$ by solving the set of differential 
equations, Eqs. (5.55)-(5.57). Define

$$
I_{+}(t) \equiv I_{y}(t)+{ }_{1} I_{z}(t)
$$

From the second and third equations of Eqs. (5.55)-(5.57), one obtains

$$
\frac{d}{d t}\left\langle I_{+}(t)\right\rangle+\left\{\gamma_{1}(t)+12 \alpha[B(t)-\Delta]\right\}\left\langle I_{+}(t)\right\rangle=-\left[\omega_{z}+\gamma_{2}(t)\right]\left\langle I_{x}(t)\right\rangle+1 \frac{1}{2} A(t)
$$

The homogeneous solution of Eq. (5.63) is

$$
\left\langle I_{+}(t)\right\rangle=\left\langle I_{+}(0)\right\rangle \mathrm{e}^{-\int_{0}^{t} d \tau\left\{\gamma_{1}(\tau)+1 \alpha[B(\tau)-2 \Delta]\right\}},
$$

while its particular solution reads

$$
\left\langle I_{+}(t)\right\rangle=\int_{0}^{t} d \tau\left\{-\left[\omega_{z}+\gamma_{2}(\tau)\right]\left\langle I_{x}(\tau)\right\rangle+1 A(\tau)\right\} \mathrm{e}^{-\int_{\tau}^{t} d \tau^{\prime}\left\{\gamma_{1}\left(\tau^{\prime}\right)+1 \alpha\left[B\left(\tau^{\prime}\right)-2 \Delta\right]\right\}}
$$

Since

$$
\begin{aligned}
\left\langle I_{y}(t)\right\rangle= & \Re\left[\left\langle I_{+}(t)\right\rangle\right] \\
= & -\int_{0}^{t} d \tau \mathrm{e}^{-\int_{\tau}^{t} d \tau^{\prime} \gamma_{1}\left(\tau^{\prime}\right)}\left[\omega_{z}+\gamma_{2}(\tau)\right]\left\langle I_{x}(\tau)\right\rangle \cos \left\{\int_{\tau}^{t} d \tau^{\prime} 2 \alpha\left[B\left(\tau^{\prime}\right)-\Delta\right]\right\} \\
& +\frac{1}{2} \int_{0}^{t} d \tau \mathrm{e}^{-\int_{\tau}^{t} d \tau^{\prime} \gamma_{1}\left(\tau^{\prime}\right)} A(\tau) \sin \left\{\int_{\tau}^{t} d \tau^{\prime} \alpha\left[B\left(\tau^{\prime}\right)-2 \Delta\right]\right\}
\end{aligned}
$$


and

$$
\frac{d}{d t}\left\langle I_{x}(t)\right\rangle=\omega_{z}\left\langle I_{y}(t)\right\rangle,
$$

the evolution of $\left\langle I_{x}(t)\right\rangle$ is finally governed by

$$
\begin{aligned}
\frac{d}{d t}\left\langle I_{x}(t)\right\rangle= & -\omega_{z} \int_{0}^{t} d \tau \mathrm{e}^{-\int_{\tau}^{t} d \tau^{\prime} \gamma_{1}\left(\tau^{\prime}\right)}\left[\omega_{z}+\gamma_{2}(\tau)\right]\left\langle I_{x}(\tau)\right\rangle \cos \left\{\int_{\tau}^{t} d \tau^{\prime} 2 \alpha\left[B\left(\tau^{\prime}\right)-\Delta\right]\right\} \\
& +\frac{1}{2} \omega_{z} \int_{0}^{t} d \tau \mathrm{e}^{-\int_{\tau}^{t} d \tau^{\prime} \gamma_{1}\left(\tau^{\prime}\right)} A(\tau) \sin \left\{\int_{\tau}^{t} d \tau^{\prime} 2 \alpha\left[B\left(\tau^{\prime}\right)-\Delta\right]\right\}
\end{aligned}
$$

This is the central result of this chapter. For the reader's convenience, the definitions of $\gamma_{1}(t), \gamma_{2}(t), A(t)$, and $B(t)$ are listed below again

$$
\begin{aligned}
\gamma_{1}(t) & \equiv \lambda^{2} \int_{0}^{t} d \tau\left\langle\tilde{\omega}_{x}^{(0)}(0) \tilde{\omega}_{x}^{(0)}(-\tau)\right\rangle \cos \left(\omega_{z} \tau\right) \\
\gamma_{2}(t) & \equiv \lambda^{2} \int_{0}^{t} d \tau\left\{\tilde{\omega}_{x}^{(0)}(0) \tilde{\omega}_{x}^{(0)}(-\tau)\right\rangle \sin \left(\omega_{z} \tau\right) \\
A(t) & \equiv \lambda^{2} \int_{0}^{t} d \tau\left[\sum_{j} \Gamma_{j}^{2} \sin \left(\nu_{j} \tau\right)\right] \sin \left(\omega_{z} \tau\right) \\
& =-2 \lambda^{2} \int_{0}^{t} d \tau\left\{\sum_{j} \Gamma_{j}^{2} \sin \left[\nu_{j}(t-\tau)\right]\right\} \frac{1}{4} \sin \left[\omega_{z}(\tau-t)\right] \\
B(t) & \equiv \lambda^{2} \int_{0}^{t} d \tau \sum_{j} \Gamma_{j}^{2} \sin \left(\nu_{j} \tau\right)=2 \lambda^{2} \int_{0}^{t} d \tau \sum_{j} \Gamma_{j}^{2} \sin \left[\nu_{j}(t-\tau)\right]
\end{aligned}
$$




\subsection{Trapped States}

When $\alpha=0$ (i.e., ignore the dipolar order), the initial density operator becomes

$$
\rho(0)=|+\rangle_{x}\left\langle+\left.\right|_{x} \exp \left[-\beta \sum_{j} \nu_{j} b_{j}^{\dagger} b_{j}\right]\right.
$$

and Eq. (5.68) can be simplified to

$$
\frac{d}{d t}\left\langle I_{x}(t)\right\rangle=-\omega_{z} \int_{0}^{t} d \tau \mathrm{e}^{-\int_{\tau}^{t} d \tau^{\prime} \gamma_{1}\left(\tau^{\prime}\right)}\left[\omega_{z}+\gamma_{2}(\tau)\right]\left\langle I_{x}(\tau)\right\rangle
$$

For time $t$ very large, the above equation can be further simplified to

$$
\frac{d}{d t}\left\langle I_{x}(t)\right\rangle=-\omega_{z}\left(\omega_{z}+\gamma_{2}\right) \int_{0}^{t} d \tau \mathrm{e}^{-(t-\tau) \gamma_{1}}\left\langle I_{x}(\tau)\right\rangle
$$

where the constants

$$
\begin{aligned}
\gamma_{1} & \equiv \lambda^{2} \int_{0}^{\infty} d \tau\left\langle\tilde{\omega}_{x}^{(0)}(0) \tilde{\omega}_{x}^{(0)}(-\tau)\right\rangle \cos \left(\omega_{z} \tau\right) \\
\gamma_{2} & \equiv \lambda^{2} \int_{0}^{\infty} d \tau\left\langle\tilde{\omega}_{x}^{(0)}(0) \tilde{\omega}_{x}^{(0)}(-\tau)\right\rangle \sin \left(\omega_{z} \tau\right)
\end{aligned}
$$

Solving with Laplace transform, one can show that, if $2 \sqrt{\omega_{z}\left(\omega_{z}+\gamma_{2}\right)}>\gamma_{1},\left\langle I_{x}(t)\right\rangle$ will be an exponentially damped sinusoid function described by

$$
\frac{d^{2}}{d t^{2}}\left\langle I_{x}(t)\right\rangle+\gamma_{1} \frac{d}{d t}\left\langle I_{x}(t)\right\rangle+\omega_{z}\left(\omega_{z}+\gamma_{2}\right)\left\langle I_{x}(t)\right\rangle=0
$$


If we assume the resulting equation to be valid also in the case of small $\omega_{z}$, then after reaching the critical condition

$$
2 \sqrt{\omega_{z}\left(\omega_{z}+\gamma_{2}\right)}=\gamma_{1}
$$

the decay of $\left\langle I_{x}(t)\right\rangle$ would change from a damped oscillation to a mere exponential behavior (Chapter 4). Furthermore, the lifetime of the exponential decay would become larger and larger. This is analogous to the inertialess motion of an oscillator moving very slowly from its initial nonequilibrium position. Thus for $\omega_{z}$ tending to be zero, the state with positive polarization in the $x$ direction would be a virtually stable state (trapped state). Therefore, keeping $\omega_{z}$ small or $\lambda$ large allows us to discover a trapping mechanism distinct from that of the standard spin relaxation process.

The above simple analysis is confirmed by numerically evaluating the transverse magnetization $\left\langle I_{x}(t)\right\rangle$ and reaction field $\left\langle\tilde{\omega}_{x}^{(R)}(t)\right\rangle$ according to Eqs. (5.68) and (5.25), respectively. Here the spectral density is assumed to have the following functional form

$$
J(\nu) \equiv \lambda^{2} \sum_{j} \Gamma_{j}^{2} \delta\left(\nu-\nu_{j}\right)=\frac{\lambda^{2} c}{\nu_{c}^{2}} \nu \exp \left(-\nu / \nu_{c}\right)
$$

where $\delta$ is the Dirac delta function and $\nu_{c}$ is the cut-off frequency. The results are depicted respectively in Figs. 4.3 (Chapter 4), 4.4 (Chapter 4), and 5.1for various values of $\omega_{z}, \lambda$, and $\alpha$. 

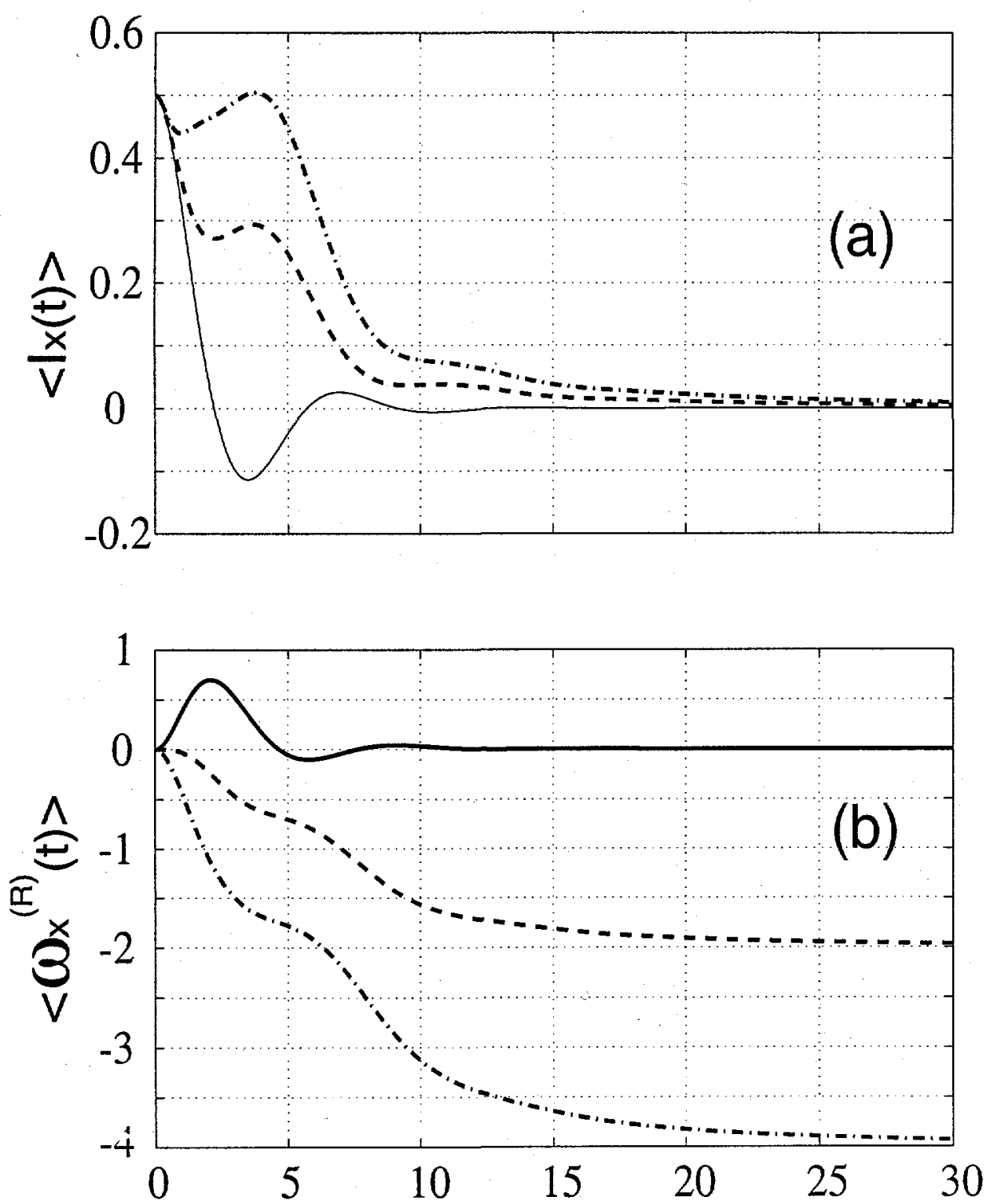

Figure 5.1: The evolution of (a) the transverse magnetization $\left\langle I_{x}(t)\right\rangle$ and (b) the reaction field $\left\langle\tilde{\omega}_{x}^{(R)}(t)\right\rangle$, obtained by numerically solving Eqs. (5.68) and (5.25), respectively. The dipolar-order parameter $\alpha=0$ (solid line), -0.5 (dash line), and -1 (dash-dot line). Other parameters used are: $\omega_{z}=1, \lambda=1,\left\langle I_{x}(0)\right\rangle=0.5$, cut-off frequency $\nu_{c}=0.5$, and $c=1$. 


\subsection{Two-Dimensional Spin-Boson Hamiltonian}

Consider the following two-dimensional spin-boson Hamiltonian

$$
\mathcal{H}=-\omega_{z} I_{z}-\lambda_{x} \omega_{x} I_{x}-\lambda_{y} \omega_{y} I_{y}+\sum_{j} \nu_{x j} b_{x j}^{\dagger} b_{x j}+\sum_{j} \nu_{y j} b_{y j}^{\dagger} b_{y j}
$$

where

$$
\begin{aligned}
\omega_{x} & \equiv \sum_{j} \omega_{x j}, \\
\omega_{x j} & \equiv \Gamma_{x j}\left(b_{x j}+b_{x j}^{\dagger}\right), \\
\omega_{y} & \equiv \sum_{j} \omega_{y j}, \\
\omega_{y j} & \equiv \Gamma_{y j}\left(b_{y j}+b_{y j}^{\dagger}\right) .
\end{aligned}
$$

The corresponding set of Heisenberg equations reads

$$
\begin{aligned}
\frac{d}{d t} I_{x}(t) & =\omega_{z} I_{y}(t)-\lambda_{y} \omega_{y}(t) I_{z}(t), \\
\frac{d}{d t} I_{y}(t) & =-\omega_{z} I_{x}(t)+\lambda_{x} \omega_{x}(t) I_{z}(t), \\
\frac{d}{d t} I_{z}(t) & =-\lambda_{x} \omega_{x}(t) I_{y}(t)+\lambda_{y} \omega_{y}(t) I_{x}(t), \\
\frac{d^{2}}{d t^{2}} \omega_{x j}(t)+\nu_{x j}^{2} \omega_{x j}(t) & =2 \lambda_{x} \nu_{x j} \Gamma_{x j}^{2} I_{x}(t), \\
\frac{d^{2}}{d t^{2}} \omega_{y j}(t)+\nu_{y j}^{2} \omega_{y j}(t) & =2 \lambda_{y} \nu_{y j} \Gamma_{y j}^{2} I_{y}(t) .
\end{aligned}
$$


After a $\pi / 2$ pulse along $-y$ axis, the initial density operator can be written as

$$
\rho(0) \propto|+\rangle_{x}\left\langle+\left.\right|_{x} \exp \left[-\beta\left(\lambda_{x} \alpha \omega_{x}+\sum_{j} \nu_{x j} b_{x j}^{\dagger} b_{x j}+\sum_{j} \nu_{y j} b_{y j}^{\dagger} b_{y j}\right)\right]\right.
$$

where $\alpha$ is a parameter used to study the dependence of spin dynamics on the initial density operator, especially on the magnitude of the dipolar order.

Define the following polaronic transform:

$$
\begin{aligned}
\tilde{b}_{x j} & \equiv b_{x j}+\frac{\lambda_{x} \Gamma_{x j}}{\nu_{x j}} \alpha, \\
\tilde{\omega}_{x j} & \equiv \Gamma_{x j}\left(\tilde{b}_{x j}+\tilde{b}_{x j}^{\dagger}\right), \\
\tilde{\omega}_{x} & \equiv \sum_{j} \tilde{\omega}_{x j} .
\end{aligned}
$$

The initial condition of Eq. (5.87) expressed in terms of the new operators is

$$
\rho(0) \propto|+\rangle_{x}\left\langle+\left.\right|_{x} \exp \left[-\beta\left(\sum_{j} \nu_{x j} \tilde{b}_{x j}^{\dagger} \tilde{b}_{x j}+\sum_{j} \nu_{y j} b_{y j}^{\dagger} b_{y j}\right)\right]\right.
$$

In the new reference system, the set of Heisenberg equations of Eqs. (5.82)-(5.85) 
becomes

$$
\begin{aligned}
\frac{d}{d t} I_{x}(t) & =\omega_{z} I_{y}(t)-\lambda_{y} \omega_{y}(t) I_{z}(t) \\
\frac{d}{d t} I_{y}(t) & =-\omega_{z} I_{x}(t)-2 \Delta \alpha I_{z}(t)+\lambda_{x} \tilde{\omega}_{x}(t) I_{z}(t), \\
\frac{d}{d t} I_{z}(t) & =2 \Delta \alpha I_{y}(t)-\lambda_{x} \tilde{\omega}_{x}(t) I_{y}(t)+\lambda_{y} \omega_{y}(t) I_{x}(t), \\
\frac{d^{2}}{d t^{2}} \tilde{\omega}_{x j}(t)+\nu_{x j}^{2} \tilde{\omega}_{x j}(t) & =2 \lambda_{x} \nu_{x j} \Gamma_{x j}^{2}\left[I_{x}(t)+\alpha\right] \\
\frac{d^{2}}{d t^{2}} \omega_{y j}(t)+\nu_{y j}^{2} \omega_{y j}(t) & =2 \lambda_{y} \nu_{y j} \Gamma_{y j}^{2} I_{y}(t)
\end{aligned}
$$

where

$$
\Delta \equiv \lambda_{x}^{2} \sum_{j} \frac{\Gamma_{x j}^{2}}{\nu_{x j}} .
$$

Solving Eqs. (5.95) and (5.96) one obtains

$$
\begin{aligned}
\tilde{\omega}_{x}(t) & =\tilde{\omega}_{x}^{(0)}(t)+\tilde{\omega}_{x}^{(R)}(t) \\
\omega_{x}^{(0)}(t) & =\sum_{j} \Gamma_{x j}\left(b_{x j} \mathrm{e}^{-1 \nu_{x j} t}+b_{x j}^{\dagger} \mathrm{e}^{1 \nu_{x j} t}\right) \\
\tilde{\omega}_{x}^{(R)}(t) & =2 \lambda_{x} \int_{0}^{t} d \tau\left\{\sum_{j} \Gamma_{x j}^{2} \sin \left[\nu_{x j}(t-\tau)\right]\right\}\left[I_{x}(\tau)+\alpha\right] \\
\tilde{\omega}_{y}(t) & =\tilde{\omega}_{y}^{(0)}(t)+\tilde{\omega}_{y}^{(R)}(t) \\
\omega_{y}^{(0)}(t) & =\sum_{j} \Gamma_{y j}\left(b_{y j} \mathrm{e}^{-1 \nu_{y j} t}+b_{y j}^{\dagger} \mathrm{e}^{i \nu_{y j} t}\right) \\
\tilde{\omega}_{y}^{(R)}(t) & =2 \lambda_{y} \int_{0}^{t} d \tau\left\{\sum_{j} \Gamma_{y j}^{2} \sin \left[\nu_{y j}(t-\tau)\right]\right\} I_{y}(\tau) .
\end{aligned}
$$


The terms $\tilde{\omega}_{x}^{(0)}(t)$ and $\tilde{\omega}_{y}^{(0)}(t)$ describe the free fluctuations of the lattice in the absence of the interaction with the spin-1/2 system (the direct fields), while the terms $\tilde{\omega}_{x}^{(R)}(t)$ and $\tilde{\omega}_{y}^{(R)}(t)$ are the reaction fields. Insert these solutions into the set of Heisenberg equations in the new reference frame, one finally reaches:

$$
\begin{aligned}
\frac{d}{d t} I_{x}(t)= & \omega_{z} I_{y}(t)-\lambda_{y}\left[\tilde{\omega}_{y}^{(0)}(t) I_{z}(t)\right]_{S} \\
& -2 \lambda_{y}^{2} \int_{0}^{t} d \tau\left\{\sum_{j} \Gamma_{y j}^{2} \sin \left[\nu_{y j}(t-\tau)\right]\right\}\left[I_{y}(\tau) I_{z}(t)\right]_{S} \\
\frac{d}{d t} I_{y}(t)= & -\omega_{z} I_{x}(t)-2 \Delta \alpha I_{z}(t)+\lambda_{x}\left[\tilde{\omega}_{x}^{(0)}(t) I_{z}(t)\right]_{S} \\
& +2 \lambda_{x}^{2} \int_{0}^{t} d \tau\left\{\sum_{j} \Gamma_{x j}^{2} \sin \left[\nu_{x j}(t-\tau)\right]\right\}\left[\left(I_{x}(\tau)+\alpha\right) I_{z}(t)\right]_{S} \\
\frac{d}{d t} I_{z}(t)= & 2 \Delta \alpha I_{y}(t)-\lambda_{x}\left[\tilde{\omega}_{x}^{(0)}(t) I_{y}(t)\right]_{S} \\
& -2 \lambda_{x}^{2} \int_{0}^{t} d \tau\left\{\sum_{j} \Gamma_{x j}^{2} \sin \left[\nu_{x j}(t-\tau)\right]\right\}\left[\left(I_{x}(\tau)+\alpha\right) I_{y}(t)\right]_{S}+\lambda_{y}\left[\tilde{\omega}_{y}^{(0)}(t) I_{x}(t)\right]_{S} \\
& +2 \lambda_{y}^{2} \int_{0}^{t} d \tau\left\{\sum_{j} \Gamma_{y j}^{2} \sin \left[\nu_{y j}(t-\tau)\right]\right\}\left[I_{y}(\tau) I_{x}(t)\right]_{S}
\end{aligned}
$$

where

$$
[a b]_{S} \equiv \frac{1}{2}(a b+b a)
$$

for two arbitrary operators $a$ and $b$. Equations (5.104)-(5.106) are formally exact, and are the starting point for perturbation treatment. 
From Eqs. (5.104)-(5.106), one gets the following zeroth-order approximations

$$
\begin{aligned}
\frac{d}{d t} I_{x}(t) & =\omega_{z} I_{y}(t), \\
\frac{d}{d t} I_{y}(t) & =-\omega_{z} I_{x}(t), \\
\frac{d}{d t} I_{z}(t) & =0 .
\end{aligned}
$$

Solving the set of differential equations, one obtains the zeroth-order solutions

$$
\begin{aligned}
& I_{x}(t)=I_{x}(0) \cos \omega_{z} t+I_{y}(0) \sin \omega_{z} t \\
& I_{y}(t)=I_{y}(0) \cos \omega_{z} t-I_{x}(0) \sin \omega_{z} t \\
& I_{z}(t)=I_{z}(0)
\end{aligned}
$$

Therefore, the two-time operators, when evaluated at the zeroth order, become

$$
\begin{gathered}
{\left[I_{x}(\tau) I_{z}(t)\right]_{S}=0} \\
{\left[I_{x}(\tau) I_{y}(t)\right]_{S}=\frac{1}{4} \sin \left[\omega_{z}(\tau-t)\right] .} \\
{\left[I_{y}(\tau) I_{z}(t)\right]_{S}=0,} \\
{\left[I_{y}(\tau) I_{x}(t)\right]_{S}=-\frac{1}{4} \sin \left[\omega_{z}(\tau-t)\right] .}
\end{gathered}
$$


The first order approximation to Eqs. (5.104)-(5.106) reads

$$
\begin{aligned}
\frac{d}{d t} I_{x}(t) & =\omega_{z} I_{y}(t)-\lambda_{y}\left[\tilde{\omega}_{y}^{(0)}(t) I_{z}(t)\right]_{S} \\
\frac{d}{d t} I_{y}(t) & =-\omega_{z} I_{x}(t)+\lambda_{x}\left[\tilde{\omega}_{x}^{(0)}(t) I_{z}(t)\right]_{S} \\
\frac{d}{d t} I_{z}(t) & =-\lambda_{x}\left[\tilde{\omega}_{x}^{(0)}(t) I_{y}(t)\right]_{S}+\lambda_{y}\left[\tilde{\omega}_{y}^{(0)}(t) I_{x}(t)\right]_{S}
\end{aligned}
$$

Solving the set of differential equations, one obtains the first-order solutions

$$
\begin{aligned}
I_{x}(t)= & I_{x}(0) \cos \left(\omega_{z} t\right)+I_{y}(0) \sin \left(\omega_{z} t\right)+\lambda_{x} \int_{0}^{t} d \tau \sin \left[\omega_{z}(t-\tau)\right]\left[\tilde{\omega}_{x}^{(0)}(\tau) I_{z}(\tau)\right]_{S} \\
& -\lambda_{y} \int_{0}^{t} d \tau \cos \left[\omega_{z}(t-\tau)\right]\left[\tilde{\omega}_{y}^{(0)}(\tau) I_{z}(\tau)\right]_{S} \\
I_{y}(t)= & I_{y}(0) \cos \left(\omega_{z} t\right)-I_{x}(0) \sin \left(\omega_{z} t\right)+\lambda_{y} \int_{0}^{t} d \tau \sin \left[\omega_{z}(t-\tau)\right]\left[\tilde{\omega}_{y}^{(0)}(\tau) I_{z}(\tau)\right]_{S} \\
& +\lambda_{x} \int_{0}^{t} d \tau \cos \left[\omega_{z}(t-\tau)\right]\left[\tilde{\omega}_{x}^{(0)}(\tau) I_{z}(\tau)\right]_{S} \\
I_{z}(t)= & I_{z}(0)-\lambda_{x} \int_{0}^{t} d \tau\left[\tilde{\omega}_{x}^{(0)}(\tau) I_{y}(\tau)\right]_{S}+\lambda_{y} \int_{0}^{t} d \tau\left[\tilde{\omega}_{y}^{(0)}(\tau) I_{x}(\tau)\right]_{S}
\end{aligned}
$$

Insert the zeroth-order and first-order solutions into Eqs. (5.104)-(5.106) and then take ensemble average by using the key assumptions that $\tilde{\omega}_{x}^{(R)}(t) \approx\left\langle\tilde{\omega}_{x}^{(R)}(t)\right\rangle$ and $\tilde{\omega}_{y}^{(R)}(t) \approx\left\langle\tilde{\omega}_{y}^{(R)}(t)\right\rangle$. The time evolution of $I_{x}(t-\tau), I_{y}(t-\tau)$ and $I_{z}(t-\tau)$ from $t-\tau$ up to $t$, within their respective time-convoluted expressions, must be evaluated at the zeroth order in $\lambda$ to have a consistent second-order perturbation treatment. This 
means

$$
\begin{aligned}
\left\langle I_{x}(t-\tau)\right\rangle & \approx\left\langle I_{x}(t)\right\rangle \cos \left(\omega_{z} \tau\right)-\left\langle I_{y}(t)\right\rangle \sin \left(\omega_{z} \tau\right) \\
\left\langle I_{y}(t-\tau)\right\rangle & \approx\left\langle I_{y}(t)\right\rangle \cos \left(\omega_{z} \tau\right)+\left\langle I_{x}(t)\right\rangle \sin \left(\omega_{z} \tau\right) \\
\left\langle I_{z}(t-\tau)\right\rangle & \approx\left\langle I_{z}(t)\right\rangle .
\end{aligned}
$$

By adopting the above approximations, one finally reaches

$$
\begin{aligned}
\frac{d}{d t}\left\langle I_{x}(t)\right\rangle= & \omega_{z}\left\langle I_{y}(t)\right\rangle-\gamma_{1 y}\left\langle I_{x}(t)\right\rangle+\gamma_{2 y}\left\langle I_{y}(t)\right\rangle, \\
\frac{d}{d t}\left\langle I_{y}(t)\right\rangle= & -\omega_{z}\left\langle I_{x}(t)\right\rangle-2 \Delta \alpha\left\langle I_{z}(t)\right\rangle-\gamma_{1 x}(t)\left\langle I_{y}(t)\right\rangle \\
& -\gamma_{2 x}(t)\left\langle I_{x}(t)\right\rangle+2 \alpha B_{x}(t)\left\langle I_{z}(t)\right\rangle, \\
\frac{d}{d t}\left\langle I_{z}(t)\right\rangle= & 2 \Delta \alpha\left\langle I_{y}(t)\right\rangle-\gamma_{1 x}(t)\left\langle I_{z}(t)\right\rangle+\frac{1}{2} A(t) \\
& -2 \alpha B_{x}(t)\left\langle I_{y}(t)\right\rangle-\gamma_{1 y}\left\langle I_{z}(t)\right\rangle .
\end{aligned}
$$


where $\gamma_{1 x}(t), \gamma_{2 x}(t), \gamma_{1 y}(t), \gamma_{2 y}(t), A(t), B_{x}(t)$, and $B_{y}(t)$ are defined as

$$
\begin{aligned}
\gamma_{1 x}(t) \equiv & \lambda_{x}^{2} \int_{0}^{t} d \tau\left\langle\tilde{\omega}_{x}^{(0)}(0) \tilde{\omega}_{x}^{(0)}(-\tau)\right\rangle \cos \left(\omega_{z} \tau\right), \\
\gamma_{2 x}(t) \equiv & \lambda_{x}^{2} \int_{0}^{t} d \tau\left\langle\tilde{\omega}_{x}^{(0)}(0) \tilde{\omega}_{x}^{(0)}(-\tau)\right\rangle \sin \left(\omega_{z} \tau\right), \\
\gamma_{1 y}(t) \equiv & \lambda_{y}^{2} \int_{0}^{\infty} d \tau\left\langle\tilde{\omega}_{y}^{(0)}(0) \tilde{\omega}_{y}^{(0)}(-\tau)\right\rangle \cos \left(\omega_{z} \tau\right), \\
\gamma_{2 y}(t) \equiv & \lambda_{y}^{2} \int_{0}^{\infty} d \tau\left\langle\tilde{\omega}_{y}^{(0)}(0) \tilde{\omega}_{y}^{(0)}(-\tau)\right\rangle \sin \left(\omega_{z} \tau\right) \\
A(t) \equiv & \lambda_{x}^{2} \int_{0}^{t} d \tau\left[\sum_{j} \Gamma_{x j}^{2} \sin \left(\nu_{x j} \tau\right)\right] \sin \left(\omega_{z} \tau\right) \\
& +\lambda_{y}^{2} \int_{0}^{t} d \tau\left[\sum_{j} \Gamma_{y j}^{2} \sin \left(\nu_{y j} \tau\right)\right] \sin \left(\omega_{z} \tau\right), \\
B_{x}(t) \equiv & \lambda_{x}^{2} \int_{0}^{t} d \tau \sum_{j} \Gamma_{x j}^{2} \sin \left[\nu_{x j}(t-\tau)\right] \\
B_{y}(t) \equiv & \lambda_{y}^{2} \int_{0}^{t} d \tau \sum_{j} \Gamma_{y j}^{2} \sin \left[\nu_{y j}(t-\tau)\right] .
\end{aligned}
$$

This is the central result of this section.

By assuming the functional forms of the spectral density

$$
\begin{aligned}
& J_{x}(\nu) \equiv \lambda_{x}^{2} \sum_{j} \Gamma_{x j}^{2} \delta\left(\nu-\nu_{x j}\right), \\
& J_{y}(\nu) \equiv \lambda_{y}^{2} \sum_{j} \Gamma_{y j}^{2} \delta\left(\nu-\nu_{y j}\right),
\end{aligned}
$$

one can then numerically solve the coupled differential equations of Eqs. (5.127)- 
(5.129) with the following initial condition

$$
\left\langle I_{x}(0)\right\rangle=\frac{1}{2},\left\langle I_{y}(0)\right\rangle=\left\langle I_{z}(0)\right\rangle=0 .
$$

The results are similar to those depicted in Figs. 4.3 (Chapter 4), 4.4 (Chapter 4), and 5.1 for various values of $\omega_{z}, \lambda$, and $\alpha$.

\subsection{Conclusion}

In this chapter, we focus our attention on the interesting spin dynamics described by the spin-boson Hamiltonian. We find an analytical result based on the polaronic transform and a second-order perturbation treatment. The adoption of the polaronic transform results in a component of the "magnetic field" with an $x$ component depending on the state of the spin. In the weak coupling regime (i.e., large $\omega_{z}$ or small $\lambda),\left\langle I_{x}(t)\right\rangle$ exhibits damped oscillations around the standard equilibrium $\left\langle I_{x}(t)\right\rangle=0$. Upon decrease of $\omega_{z}$ or increases of $\lambda$ and $\alpha$ (dipolar order), a transition to the overdamped motion takes place. It is concluded that a reliable analysis of a general fluctuation-dissipation process requires a proper inclusion of the reaction field, in addition to the direct field. The other striking consequences of the reaction field will be discussed in the next chapter under strong semiclassical assumptions. 


\section{Bibliography}

[1] A. Abragam, Principles of Nuclear Magnetism (Clarendon, Oxford, 1961).

[2] P. W. Anderson and P. R. Weiss, Rev. Mod. Phys. 25, 269 (1953).

[3] R. Kubo and N. Hashitsume, Prog. Theor. Phys. Suppl. 46, 210 (1970).

[4] R. F. Fox, J. Math. Phys. 15, 217 (1974).

[5] A. J. Leggett, S. Chakravarty, A. T. Dorsey, M. P. A. Fisher, A. Garg and W. Zwerger, Rev. Mod. Phys. 59, 1 (1987).

[6] U. Weiss, Quantum Dissipative Systems (World Scientific, New Jersey, 1993).

[7] R. A. Harris and R. Silbey, J. Chem. Phys. 78, 7330 (1983).

[8] R. Silbey and R. A. Harris, J. Chem. Phys. 80, 2615 (1984).

[9] J. S. Bader, R. A. Kuharski, and D. Chandler, J. Chem. Phys. 93, 230 (1990).

[10] A. J. Leggett and A. Garg, Phys. Rev. Lett. 54, 857 (1985).

[11] K. Lindenberg and B. J. West, Phys. Rev. 30, 568 (1984). 
[12] T. D. Lee, F. E. Low, and D. Pines, Phys. Rev. 15, 297 (1953).

[13] C. Aslangul, N. Pottier, and D. Saint James, J. Phys. (Paris) 46, 2031 (1985).

[14] C. Aslangul, N. Pottier, and D. Saint James, J. Phys. (Paris) 47, 1657 (1986).

[15] G. W. Ford, M. Kac, and P. Mazur, J. Math. Phys. 6, 504 (1965). 


\section{Chapter 6}

\section{Hamiltonian Renormalization}

\subsection{Abstract}

The role of the reaction field (the bath motion induced by the coupling with the system) is the missing key property of current NMR relaxation theories. In this chapter, we illustrate how to carry out a semiclassical analysis of the spin-boson Hamiltonian without losing the influence of the reaction field. The semiclassical approximation allows us to simplify the analysis by replacing the quantum-mechanical dissipation process with the standard classical fluctuation-dissipation process. With such approximation, the spin dynamics can be described in terms of a set of nonlinear stochastic differential equations. The resulting unusual spin relaxation process can then be satisfactorily accounted by a reaction-field induced renormalization of the Hamiltonian of interest. 


\subsection{Introduction}

The stochastic Liouville equation (SLE) developed by Kubo [1], thanks to its elegance and simplicity, becomes very popular and is currently applied to many branches of spectroscopy, especially in the field of magnetic resonance. In the past decade, there have been some proposals to amend this theory by removing its main fault: the magnetization does not relax towards its equilibrium value corresponding to the given external Zeeman field but towards the zero value, due to the fact that the influence of the reaction field is disregarded. Kubo and Hashitsume show that if a frictional resistance is assumed to accompany the random field causing Brownian motion, the stochastic Liouville equation leads to a Fokker-Planck equation that guarantees approach to equilibrium [2]. A completely different attitude is adopted by Seshadri and Lindenberg [3]. These authors ground their analysis on a rigorous microscopic description, which leads them to the important conclusion that the phenomenological structure of the Kubo theory is always accompanied by a nonlinear dissipation term. This is a result of remarkable interest since this nonlinear dissipation also affects the spin dynamical behavior, thereby leading to effects which are in principle experimentally observable. A more phenomenological approach is followed by Stillman and Freed [4]. These authors study the rotational dynamics of a molecule within the theoretical framework of the SLE. They make the assumption that the rotor reaches a canonical equilibrium distribution, and use the detailed balance method to supplement the SLE with a conventional reaction field. 
In all these works, the system of interest reaches a canonical equilibrium distribution either as a consequence of a rigorous microscopic description or of explicit constraints stemming from statistical mechanics. In this chapter, we study the effects of the reaction field without imposing any constraint on the equilibrium distribution of the spin system, but only the canonical equilibrium condition for the initial state of the bath. We find out that the bare Hamiltonian must be replaced by a renormalized one, which results in an unusual precessional motion. In additional to the constant longitudinal local field along the $z$-axis, a component orthogonal to it, which depends on the mean value of the $x$ component of the dipole, appears.

\subsection{Semiclassical Approximation}

The Kubo theory [1] has essentially a phenomenological foundation. This is made especially clear by the so-called Kubo stochastic oscillator. This is a stochastic model, written as

$$
\frac{d}{d t} \boldsymbol{\mu}(t)=\mathrm{i}\left[\omega(t)+\omega_{\mathrm{o}}\right] \boldsymbol{\mu}(t)
$$

This means that, due to the influence of the bath, the dipole $\mu(t)$ is driven by a stochastic process as well as by the time-independent longitudirial field $\omega_{0}$. Within the Kubo picture, the random frequency $\omega(t)$ is assumed to be a colored Gaussian 
noise, whose dynamics is described by

$$
\frac{d}{d t} \omega(t)=-\gamma \omega(t)+f(t),
$$

where $f(t)$ is a white Gaussian noise, with vanishing mean value, defined by

$$
\langle f(0) f(t)\rangle=2 \gamma\left\langle\omega^{2}\right\rangle_{e q} \delta(t) .
$$

The imaginary frequency mimicks a precession process that actually takes place in a three-dimensional space.

Our theoretical investigation rests on the following microscopic Hamiltonian:

$$
\mathcal{H}=-\omega_{z} I_{z}-\omega_{x} I_{x}+\mathcal{H}_{B}
$$

where $\omega_{z}$ is a constant longitudinal field and $\omega_{x}$ is a fluctuating transverse field. This means that we restrict ourselves to study a strongly nonisotropic model in which the interaction along the $x$ direction prevails over that along the $y$ direction. The variable $\omega_{x}$ represents the thermal bath degrees of freedom interacting with the spin system and $\mathcal{H}_{B}$ drives the free motion of $\omega_{x}$. We are interested in the time evolution of the magnetic moment vector:

$$
\boldsymbol{\mu}=\left[2\left\langle I_{x}\right\rangle_{S}, 2\left\langle I_{y}\right\rangle_{S}, 2\left\langle I_{z}\right\rangle_{S}\right],
$$


which univocally describes the spin- $1 / 2$ density matrix $\left(\langle\rangle_{S}\right.$ means the average over the spin degrees of freedom). To write the equation of motion of $\mu$, we proceed as follows. First, from the Hamiltonian of Eq. (6.4), we derive the set of Langevinenberg equations providing the time evolution of $I_{x}(t), I_{y}(t), I_{z}(t)$, and $\omega_{x}(t)$. These equations also involve terms such as $\omega_{x}(t) I_{y}(t)$ and $\omega_{x}(t) I_{z}(t)$, the mean values of which in principle can not be factorized into a system and a bath part. By application of the semiclassical approximation, according to which $\omega_{x}$ is regarded as being a fluctuating $c$ number, it is natural to make this factorization assumption. We thus obtain

$$
\begin{aligned}
\frac{d}{d t} \mu_{x}(t) & =\omega_{z} \mu_{y}(t) \\
\frac{d}{d t} \mu_{y}(t) & =-\omega_{z} \mu_{x}(t)+\omega_{x} \mu_{z}(t), \\
\frac{d}{d t} \mu_{z}(t) & =-\omega_{x}(t) \mu_{y}(t) \\
\frac{d}{d t} \omega_{x}(t) & =-\Gamma \omega_{x}(t)-\Gamma \mu_{x}(t)-F(t)
\end{aligned}
$$

where the friction $\Gamma$ and the white Gaussian noise $F(t)$, with vanishing mean value, are related to each other by the standard fluctuation-dissipation relation

$$
\left\langle F\left(t_{1}\right) F\left(t_{2}\right)\right\rangle=2 \Gamma\left\langle\omega_{x}^{2}\right\rangle \delta\left(t_{1}-t_{2}\right),
$$

with

$$
\left\langle\omega_{x}^{2}\right\rangle=2 k_{B} T
$$


The semiclassical approximation allows us to simplify the problem by replacing the quantum-mechanical dissipation process with the standard classical fluctuation-dissipation process of Eq. (6.10). Equation (6.9) means that the bath variable $\omega_{x}$ is thought of as the coordinate of an overdamped oscillator, driven by the white and Gaussian stochastic force $F(t)$. This corresponds to the semiclassical approximation of a quantum-mechanical Hamiltonian of a spin- $1 / 2$ system interacting with a dissipative oscillator. The quantum mechanical oscillator is now replaced by a classical and stochastic oscillator in the overdamped regime. With a few mild assumptions, this in turn is equivalent to a spin coupled to a linear oscillator interacting with an infinite number of degrees of freedom, which may be taken to simulate a canonical thermal bath under a suitable hypothesis. Consequently, the quantum-mechanical variable $\omega_{x}$ has dissiptive properties due to the interaction with a virtually infinite number of degrees of freedom.

The new and relevant aspect is the presence of the reaction field (the term $\Gamma \mu_{x}$ ) in Eq. 6.9), which has precisely the same structure as that of a rigorous Hamiltonian description (Chapter 5). If one disregards this term, the dynamics implied by the set of Heisenberg equations, Eqs. (6.6)-(6.9), is fairly well understood. In the Markovian case (fast relaxation of the coupled oscillator, i.e., large $\Gamma$ ), the system is qualitatively well described by the Bloch equation [5]. The use of the SLE would allow us to extend the investigation to the case of non-Markovian process. 


\subsection{Fokker-Planck Equation}

The Fokker-Planck equation for the probability distribution $\pi\left(\mu_{x}, \mu_{y}, \mu_{z}, \omega_{x} ; t\right)$ corresponding to the set of Langevin equations, Eqs. (6.6)-(6.9), reads

$$
\begin{aligned}
\frac{\partial}{\partial t} \pi\left(\mu_{x}, \mu_{y}, \mu_{z}, \omega_{x} ; t\right) & =\mathcal{L} \pi\left(\mu_{x}, \mu_{y}, \mu_{z}, \omega_{x} ; t\right) \\
& \equiv\left(\mathcal{L}_{a}+\Lambda+\mathcal{L}_{I}\right) \pi\left(\mu_{x}, \mu_{y}, \mu_{z}, \omega_{x} ; t\right)
\end{aligned}
$$

where

$$
\begin{aligned}
\mathcal{L}_{a} & \equiv-\omega_{z} \mu_{y} \frac{\partial}{\partial \mu_{x}}+\omega_{z} \mu_{x} \frac{\partial}{\partial \mu_{y}} \\
\Lambda & \equiv \Gamma\left[\frac{\partial}{\partial \omega_{x}} \omega_{x}+\left\langle\omega_{x}^{2}\right\rangle \frac{\partial^{2}}{\partial \omega_{x}^{2}}\right] \\
\mathcal{L}_{I} & \equiv-\omega_{x} \mu_{z} \frac{\partial}{\partial \mu_{y}}+\omega_{x} \mu_{y} \frac{\partial}{\partial \mu_{z}}+\Gamma \mu_{x} \frac{\partial}{\partial \omega_{x}}
\end{aligned}
$$

Note that the above equations must be regarded as being an exact description of the dynamics of the whole system, i.e., system of interest and bath. This is due to the fact that we use the standard fluctuation-dissipation process of Eq. (6.10), which makes exact the corresponding multidimensional Fokker-Planck equation.

Now, we approach the problem of replacing the Langevin equations, Eqs. (6.6)(6.9), with a reduced description concerning only the system of interest, i.e., the dipole $\boldsymbol{\mu}$. This must be done in such a way as to properly take into account the role of the reaction field. First, the oscillator is driven to a canonical equilibrium distribution 
by the standard fluctuation-dissipation relation, and via the spin-bath interaction, transmits its own canonical properties to the system. As a result of this, the whole system is driven towards the following equilibrium distribution:

$$
\pi_{e q}\left(\mu_{x}, \mu_{y}, \mu_{z}, \omega_{x}\right) \propto \exp \left[-\frac{1}{2 k_{B} T}\left(\omega_{z} \mu_{z}+\omega_{x} \mu_{x}+\frac{\omega_{x}^{2}}{2}\right)\right]
$$

It is straightforward to verify that this is the stationary solution to Eq. (6.12). Such a solution natually leads one to describe the thermal bath in terms of a new variable,

$$
\tilde{\omega}_{x} \equiv \omega_{x}+\mu_{x}
$$

so that the total equilibrium distribution of Eq. (6.16) appears to be factorized into a system and a bath part, with no coupling term:

$$
\pi_{e q}\left(\mu_{x}, \mu_{y}, \mu_{z}, \tilde{\omega}_{x}\right) \propto \exp \left[-\frac{1}{2 k_{B} T}\left(\omega_{z} \mu_{z}-\frac{\mu_{x}^{2}}{2}+\frac{\tilde{\omega}_{x}^{2}}{2}\right)\right]
$$

Integrating over $\tilde{\omega}_{x}$ in Eq. (6.18), one can show that the spin part happens to be canonical with respect to the effective Hamiltonian

$$
\mathcal{H}_{\text {ren }}=\frac{1}{2}\left[\omega_{z} \mu_{z}-\frac{\mu_{x}^{2}}{2}\right] .
$$

Put in another way, the canonicity of the bath, represented by the standard fluctuation- 
dissipation process driving the overdamped oscillator in contact with the spin variable, is transmitted to the system of interest.

The renormalized Hamiltonian of Eq. (6.19) also means that the traditional prediction of usual statistical mechanics

$$
p_{e q}\left(\mu_{x}, \mu_{y}, \mu_{z}\right) \propto \exp \left[-\frac{1}{2 k_{B} T} \omega_{z} \mu_{z}\right]
$$

must be replaced by the following more reliable prediction:

$$
p_{e q}\left(\mu_{x}, \mu_{y}, \mu_{z}\right) \propto \exp \left[-\frac{1}{2 k_{B} T}\left(\omega_{z} \mu_{z}-\frac{\mu_{x}^{2}}{2}\right)\right]
$$

In the case of $\omega_{z} \gg 1 / 2$, the two equilibrium distribution, Eqs. (6.20) and (6.21), almost coincide. In the case of $\omega_{z} \ll 1 / 2$, on the contrary, one has a quite significant discrepancy, due to the term that is quadratic in $\mu_{x}$ that ultimately comes from our proper treatment of the reaction field. Such renormalized Hamiltonian leads to a dynamics under the action of a nonlinear potential which at a critical value of the lomgitudinal local field $\omega_{z}$ becomes bistable.

The above picture can also be obtained by briefly analyzing the dynamics described by the set of Langevin equations. By applying the transformation of Eq. (6.17), 
Eqs. (6.6)-(6.9) are replaced by

$$
\begin{aligned}
& \frac{d}{d t} \mu_{x}(t)=\omega_{z} \mu_{y}(t) \\
& \frac{d}{d t} \mu_{y}(t)=-\omega_{z} \mu_{x}(t)-\mu_{x}(t) \mu_{z}(t)+\tilde{\omega}_{x}(t) \mu_{z}(t) \\
& \frac{d}{d t} \mu_{z}(t)=\mu_{x}(t) \mu_{y}(t)-\tilde{\omega}_{x}(t) \mu_{y}(t) \\
& \frac{d}{d t} \tilde{\omega}_{x}(t)=-\Gamma \tilde{\omega}_{x}(t)+\omega_{z} \mu_{y}(t)-F(t)
\end{aligned}
$$

In this new reference frame, the reaction field is given by the term $\omega_{z} \mu_{y}(t)$. Thus, when the system is close to equilibrium (i.e., $\mu_{y}(t)=0$ ), this new expression of the reaction field turns out to be weak. From the first equation of this set, we see indeed that at equilibrium $\mu_{y}(t)$ must vanish, thereby making the reaction field equal to zero. In the adiabatic limit, one can regard the variable $\tilde{\omega}_{x}$ as being so fast fluctuating as to make it possible to replace it with its vanishing mean value. In this way, one gets the systematic motion of the spin system, which is described by the renormalized Hamiltonian of Eq. (6.19) and whose equation of motion are

$$
\begin{aligned}
\frac{d}{d t} \mu_{x}(t) & =\omega_{z} \mu_{y}(t), \\
\frac{d}{d t} \mu_{y}(t) & =-\omega_{z} \mu_{x}(t)-\mu_{x}(t) \mu_{z}(t), \\
\frac{d}{d t} \mu_{z}(t) & =\mu_{x}(t) \mu_{y}(t) .
\end{aligned}
$$

In the case of $\omega_{z} \ll 1 / 2$, this set of equations results in a bistable precessional motion. 
This is so because a systematic $x$ component of the reaction field appears. When this $x$ component is large enough with respect to the longitudinal field $\omega_{z}$, the dipole is prevented from executing a full precession around the $z$ axis. Therefore, this simple analysis shows the transition from the untrapped state (one precessional cone around the $z$ axis) to the trapped state (two distinct precessional cones).

\subsection{Numerical Simulation}

The numerical simulation of Eqs. (6.6)-(6.9) corresponds to a treatment of the semiclassical dynamics with no approximations, thereby fully including the reaction field. We assume that the initial condition is given by $\mu_{x}(0)=1, \mu_{y}(0)=0, \mu_{z}(0)=0$. In Fig. 6.1, we plot three typical trajectories.

The result looks quite different than expected on the basis of standard linear theories. In the initial part of the motion, $\mu_{x}$ executes fast oscillations of small amplitude around a nonvanishing mean value. Then it jumps into the symmetric state with just the opposite mean value and fluctuates for a while around the new equilibrium position. This behavior is closely reminiscent of a Kramers-like process: the coordinate of a particle moving in a double-well potential executes stochastic oscillations around the bottom of the potential well and then, from time to time, makes random jumps into the other well.

The transition from a trapped precessional state to an untrapped one implies the existence of an activation process, i.e., a very precise energy level has to be reached. It 

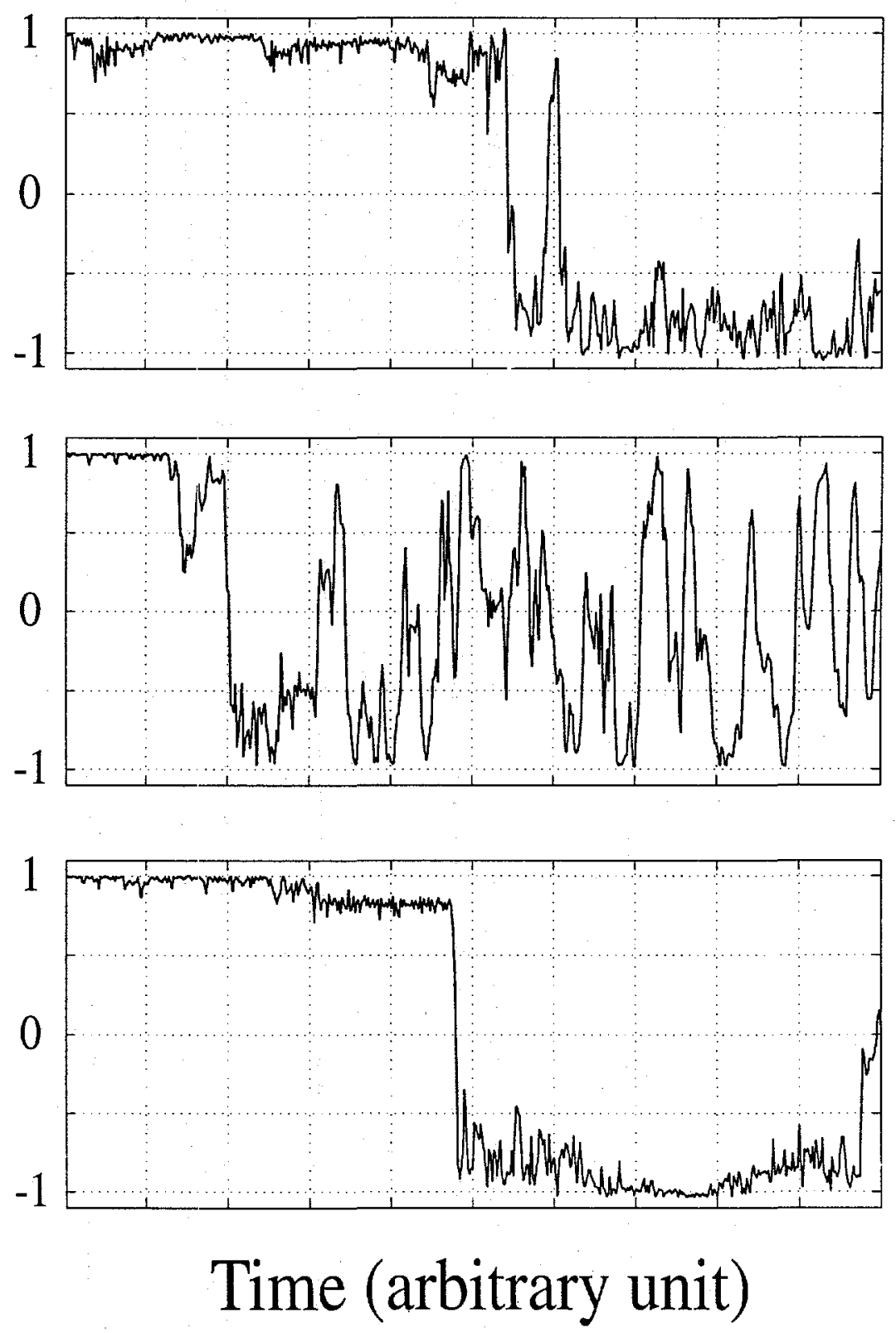

Figure 6.1: Typical trajectory of $\mu_{x}(t)$ by numerically solving the set of Langevin equations, Eqs. (€.6)-(6.9). The values of the parameters are: $\omega_{z}=\omega_{x}=1, k_{B} T=$ $0.1, \Gamma=10$. 
seems to suggest that the spin-relaxation process takes place via an energy activated process and supports our theoretical interpretation based on the renormalization of the Hamiltonian of interest. We are taught, by the theory of thermally activated processes, that if the relaxation process takes place through an escape over a potential barrier, then the relaxation process consists of two subsequent steps. At the beginning, over a very short time scale, a sort of quasistationary state is reached within the potential minima, corresponding to the attainment of a nonvanishing mean value of $\mu_{x}$. This fact by itself is quite unusual in the field of spin relaxation. Then the activation process sets in over a much more extended time scale and the final and true equilibrium state is reached. The numerical trajectory also suggests that the dynamics can not be simply described be a standard Kramer-like process. From Fig. 6.1, it is clear that the motion soon evolves into something different from the Kramer process, with $\mu_{x}$ executing large amplitude oscillations around the zero mean value. These large amplitude oscillations are quite persistent and, once brought into existence, they continue for virtually unlimited periods of time.

As a comparison, Fig. 6.2 shows some unusual trajectories of an arbitrarily tagged classical spin embedded in a simple cubic lattice containing 125 equivalent classical spins with periodic boundary condition (Chapter 7). All the spins are first treated by an ideal $90^{\circ}$ pulse along $-y$ axis and then evolves under the truncated dipolar Hamiltonian in the rotating frame precessing with the Larmor frequency. To mimick the spin-boson Hamiltonian and to make the self-trapping phenomena more obvious, 

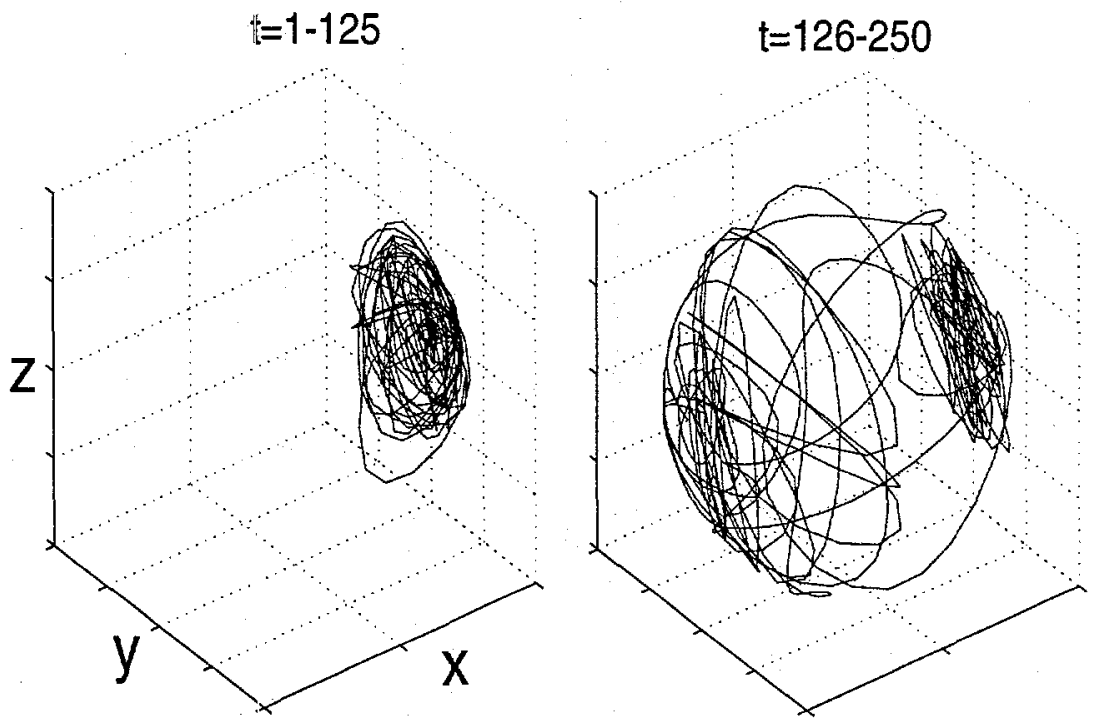

$t=251-375$

$t=376-500$
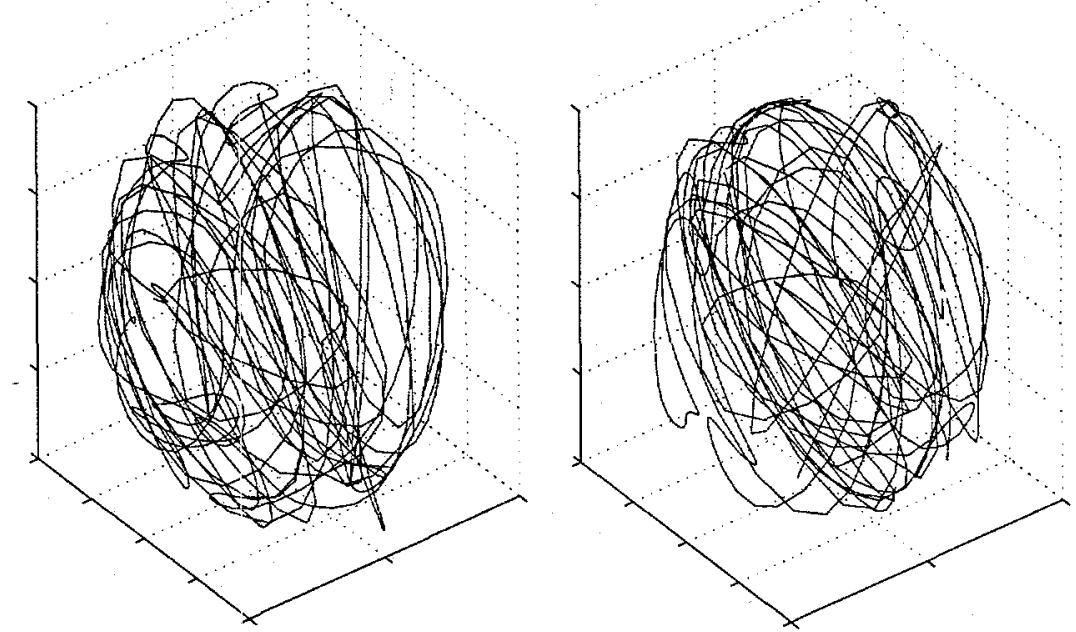

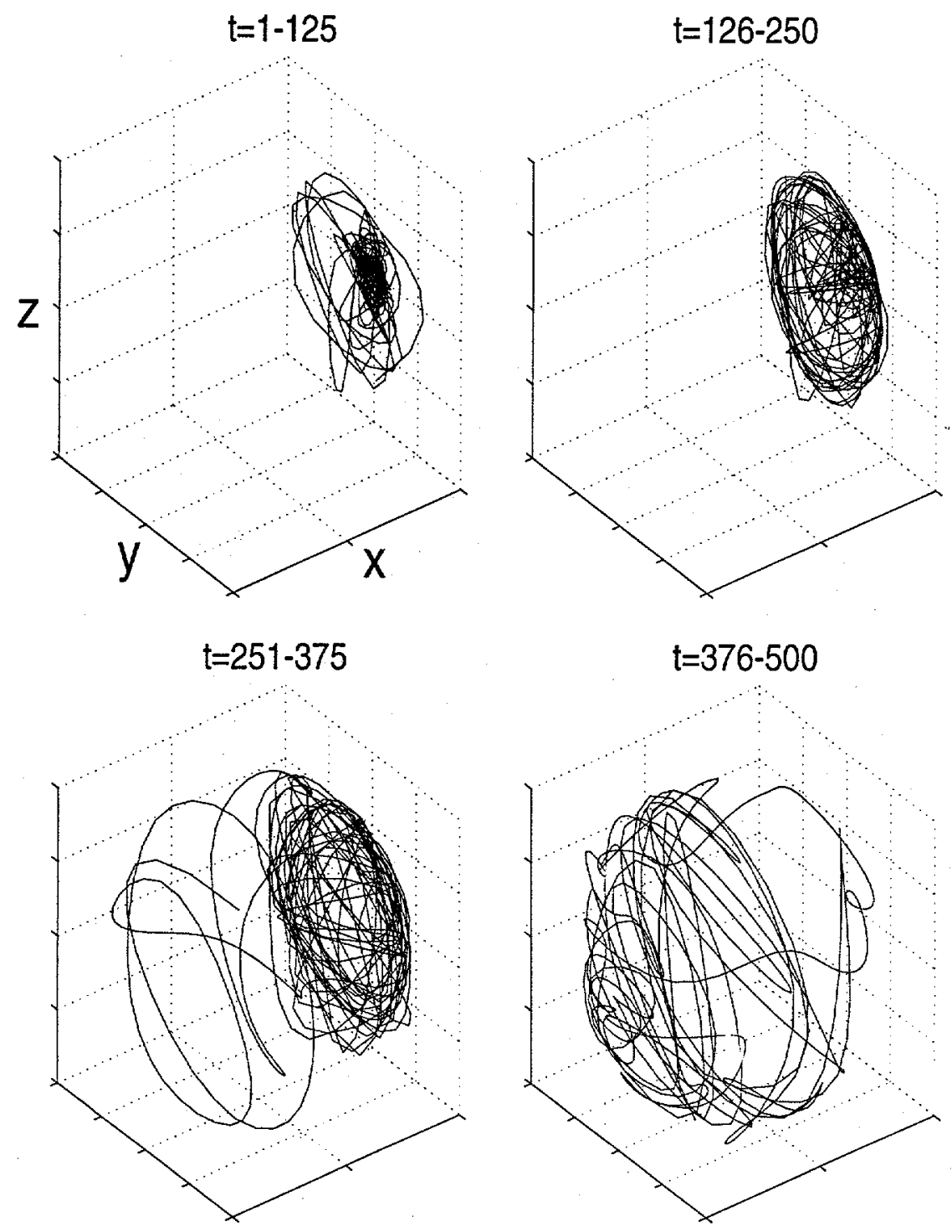

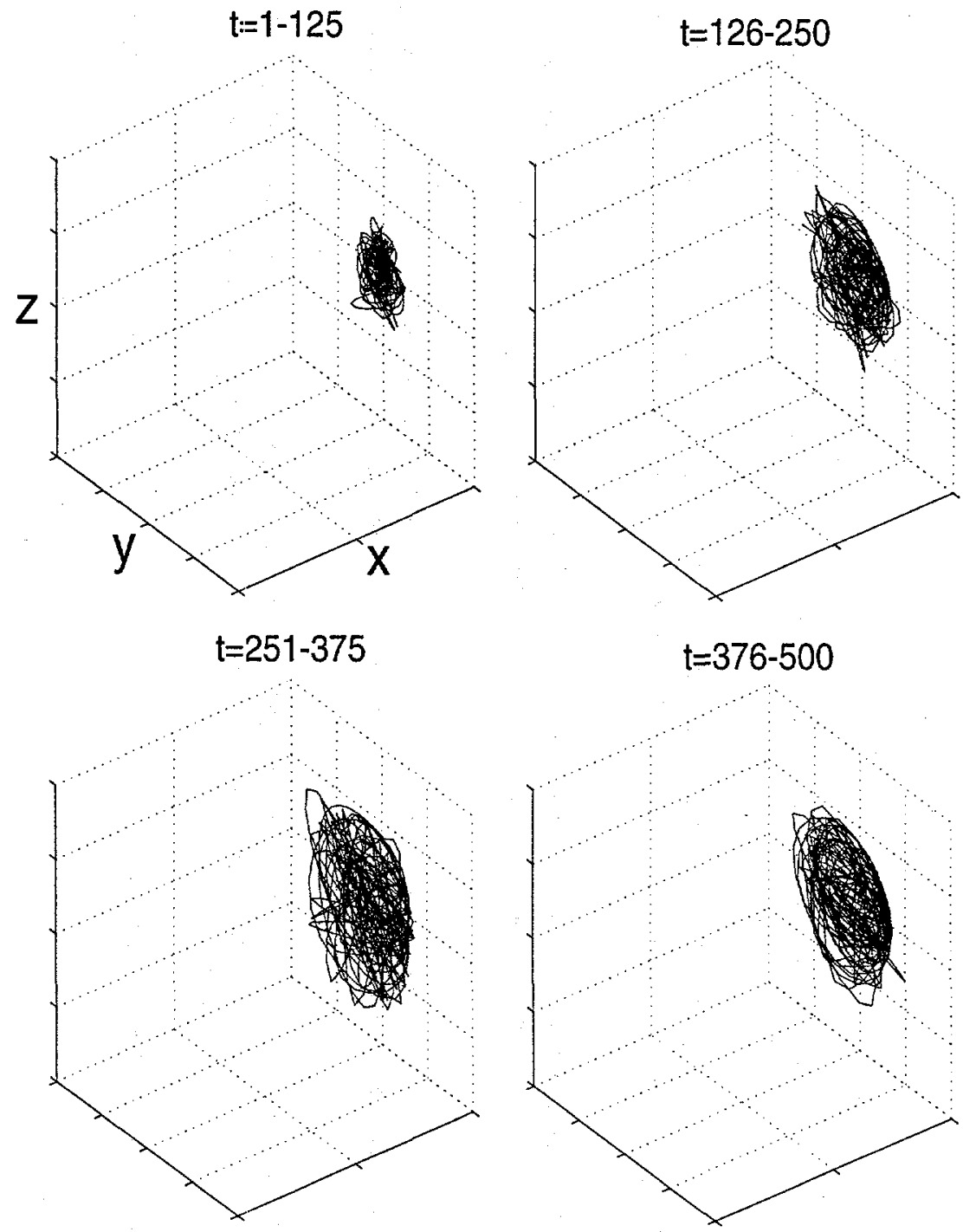

Figure 6.2: Trajectories of an arbitrarily tagged classical spin in a simple cubic lattice containing 125 equivalent classical spins with periodic boundary condition. To mimick the spin-boson Hamiltonian and to make the self-trapping phenomena more obvious, the longitudinal local field acting on the tagged spin is fixed. The dipolar coupling in the $y$ direction between the tagged spin and its neighbors is ignored, while in the $x$ direction is increased by 3 times. Time is in arbitrary unit. 
the longitudinal local field acting on the tagged spin is fixed. The dipolar coupling in the $y$ direction between the tagged spin and its neighbors is ignored, while in the $x$ direction is increased by 3 times. It shows that, as an effect of bath fluctuations, the dipole indeed jumps from one to the other metastable state in a way similar to the dynamics predicted by the semicalssical approximation of spin-boson Hamiltonian.

\subsection{Deterministic Chaos}

Before ending this chapter, it is worthwhile to briefly mention that there are other striking manifestations of semiclassical nonlinearity associated with the spin-boson Hamiltonian and the classical spin dipoles. Besides the spin self-trapping demonstrated here, there are quantum irreversibility and the fast growth of quantum mechanical uncertainity $[6,7,8,9]$. Both are provoked by semiclassical chaos, which in turn is triggered by the reaction field. The phenomenon of sensitive dependence on initial conditions is a quality that all chaotic systems definitely have. This is illustrated in Figs. 6.3, 6.4, and 6.5 for typical trajectories of $\mu_{x}(t)$ and $\mu_{z}(t)$ of an arbitrarily tagged classical spin in a simple cubic lattice containing 125 equivalent classical spins with periodic boundary condition. All the spins are first treated by an ideal pulse along $-y$ axis and then evolves under the truncated dipolar Hamiltonian in the rotating frame precessing with the Larmor frequency. The trajectory is numerically solved by explicit Runge-Kutta $(4,5)$ algorithm of Dormand-Prince [4]. All these trajectories show signs of chaos: sensitivity nonlinearly amplifies even the smallest 
difference in the initial conditions. Figure 6.3 even shows that the finite-precision numerical calculation, paradigm of computer reliability and precision, is knocked out by a simple feedback mechanicsm: the reaction field.

\subsection{Conclusion}

The semiclassical approximation to the spin-boson Hamiltonian leads to interesting nonlinear effects, triggered by the important action of the reaction field. The main effect of the reaction field is the renormalization of the Hamiltonian of the system of interest. The system equilibrium distribution turns out to be canonical with respect to the renormalized Hamiltonian of Eq. (6.19). Its direct consequence is the two-step relaxation process. Initially, the system relaxes towards one of the two quasiequilibrium states, characterized by a nonzero value of $\left\langle\mu_{x}\right\rangle$. Subsequently, the system tends to leave this state and relaxes towards the final equilibrium state characterized by $\left\langle\mu_{x}\right\rangle=0$. It is also possible to make another prediction, stemming from the fact that, for the trapped state, the transition from a precessional cone to another is an incoherent process with a mean transition time that is the inverse of such a rate. It is then expected that a stochastic resonance effect takes place when an external coherent excitation is tuned to the mean frequency of this process. 

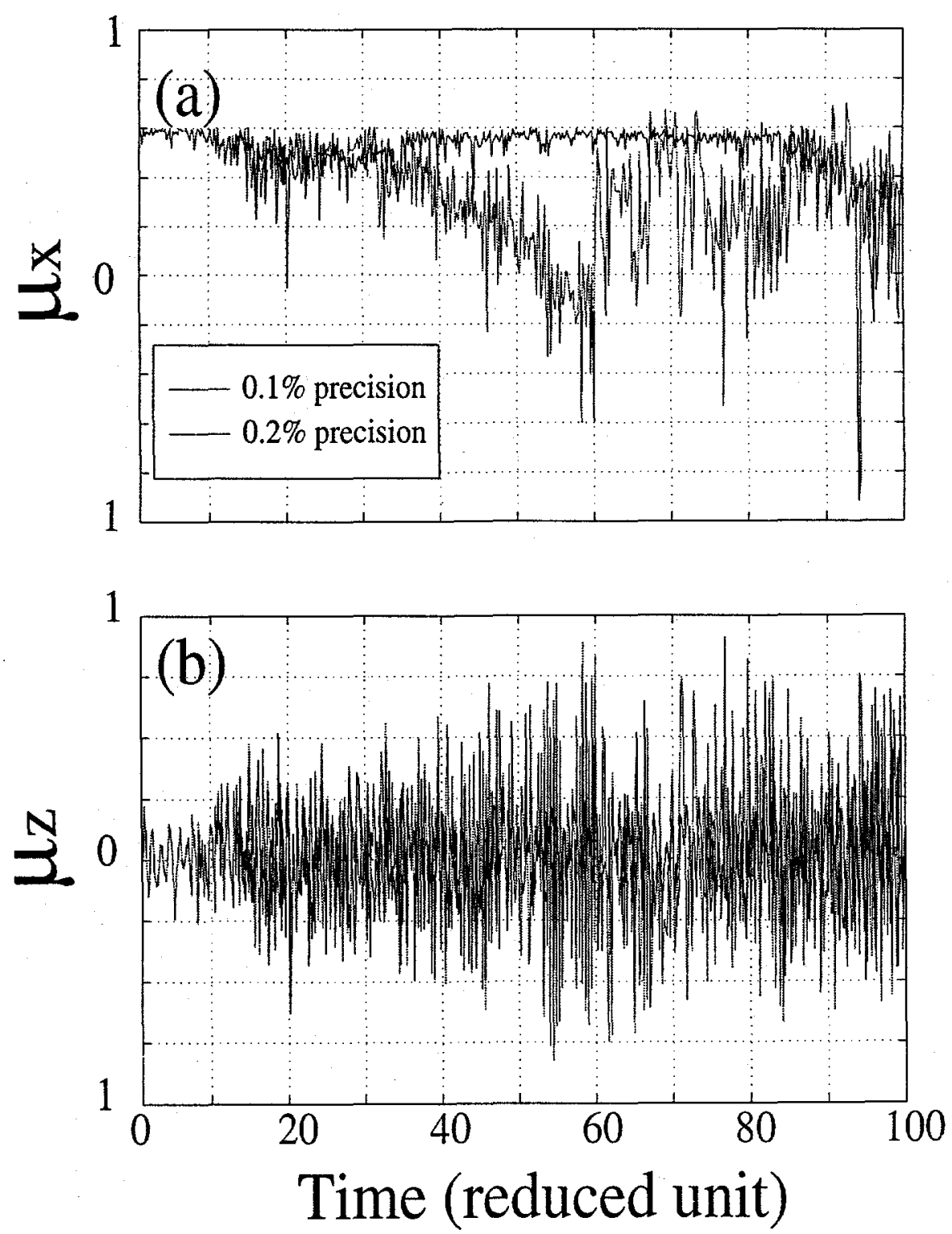

Figure 6.3: A typical trajectory of (a) $\mu_{x}(t)$ and (b) $\mu_{z}(t)$ of an arbitrarily tagged classical spin in a simple cubic lattice containing 125 equivalent classical spins with periodic boundary condition. All the spins are first treated by an ideal $90.0^{\circ}$ pulse along $-y$ axis and then evolve under the truncated dipolar Hamiltonian in the rotating frame precessing with the Larmor frequency. The trajectory is numerically solved by explicit Runge-Kutta $(4,5)$ algorithm of Dormand-Prince with relative error tolerance of $0.1 \%$ and $0.2 \%$. 
(a)

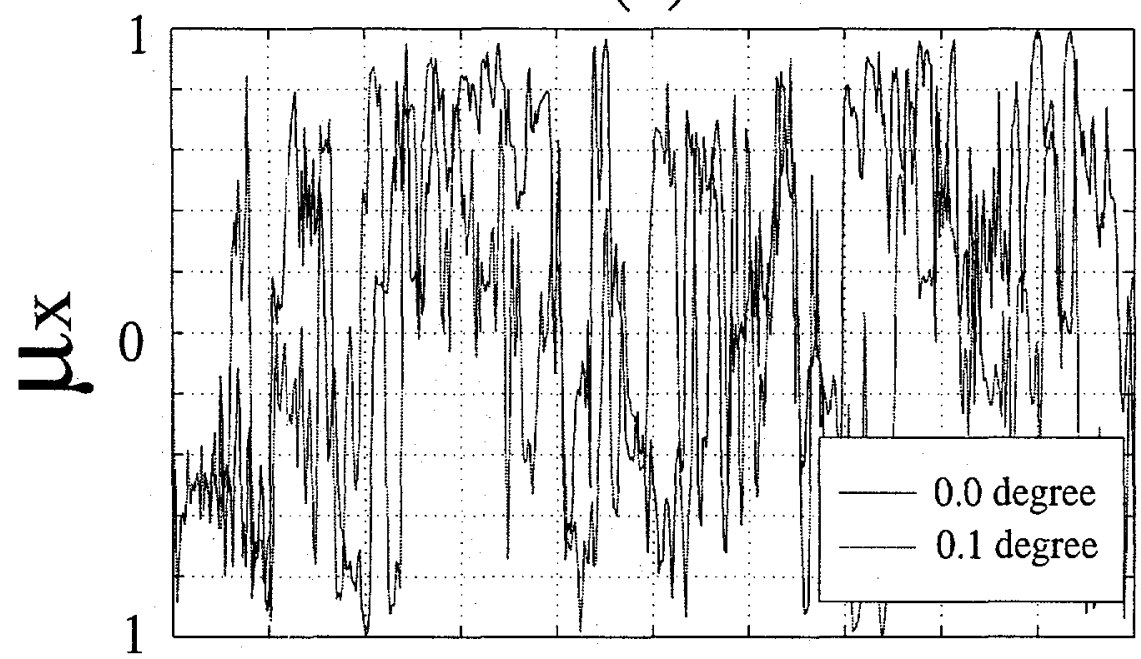

(b)

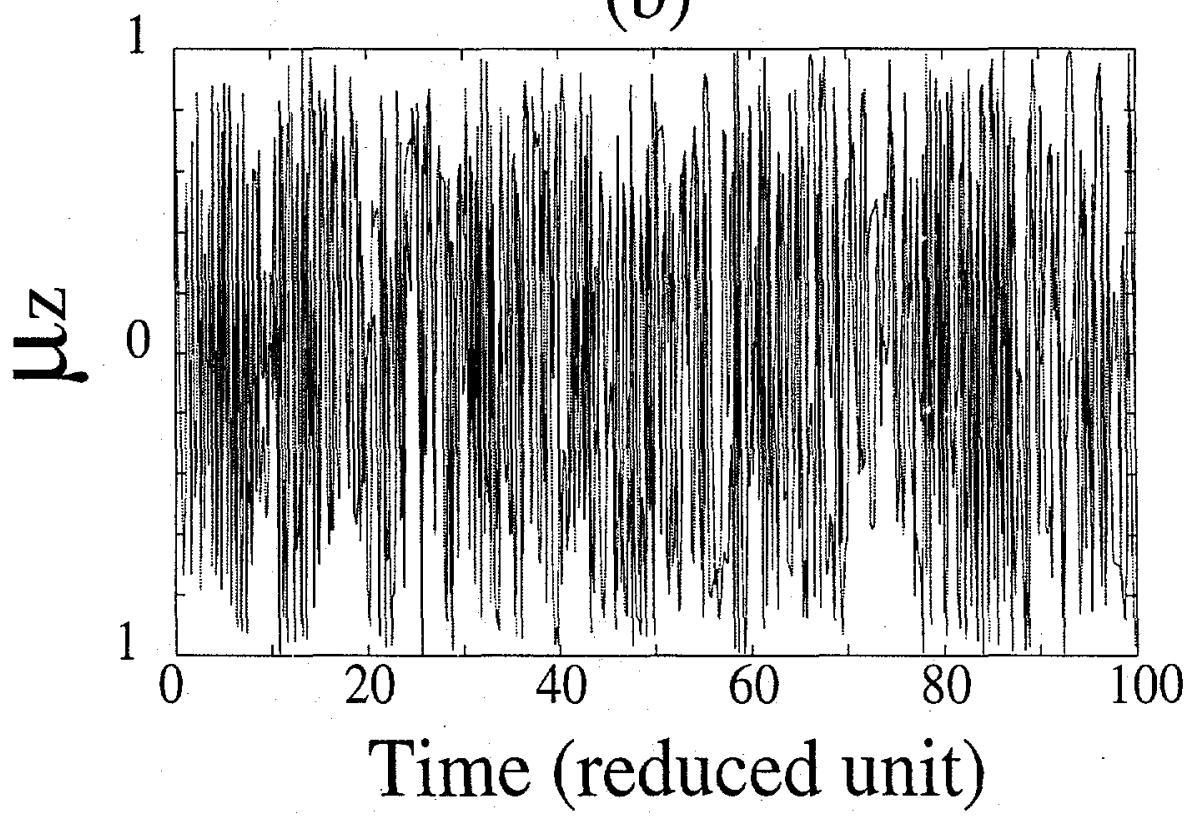

Figure 6.4: A typical trajectory of (a) $\mu_{x}(t)$ and (b) $\mu_{z}(t)$ of an arbitrarily tagged classical spin in a simple cubic lattice containing 125 equivalent classical spins with periodic boundary condition. All the spins are first treated by an ideal $0.0^{\circ}$ or $0.1^{\circ}$ pulse along $-y$ axis and then evolve under the truncated dipolar Hamiltonian in the rotating frame precessing with the Larmor frequency. The Ljapunov exponent is estimated to be 0.32 . 

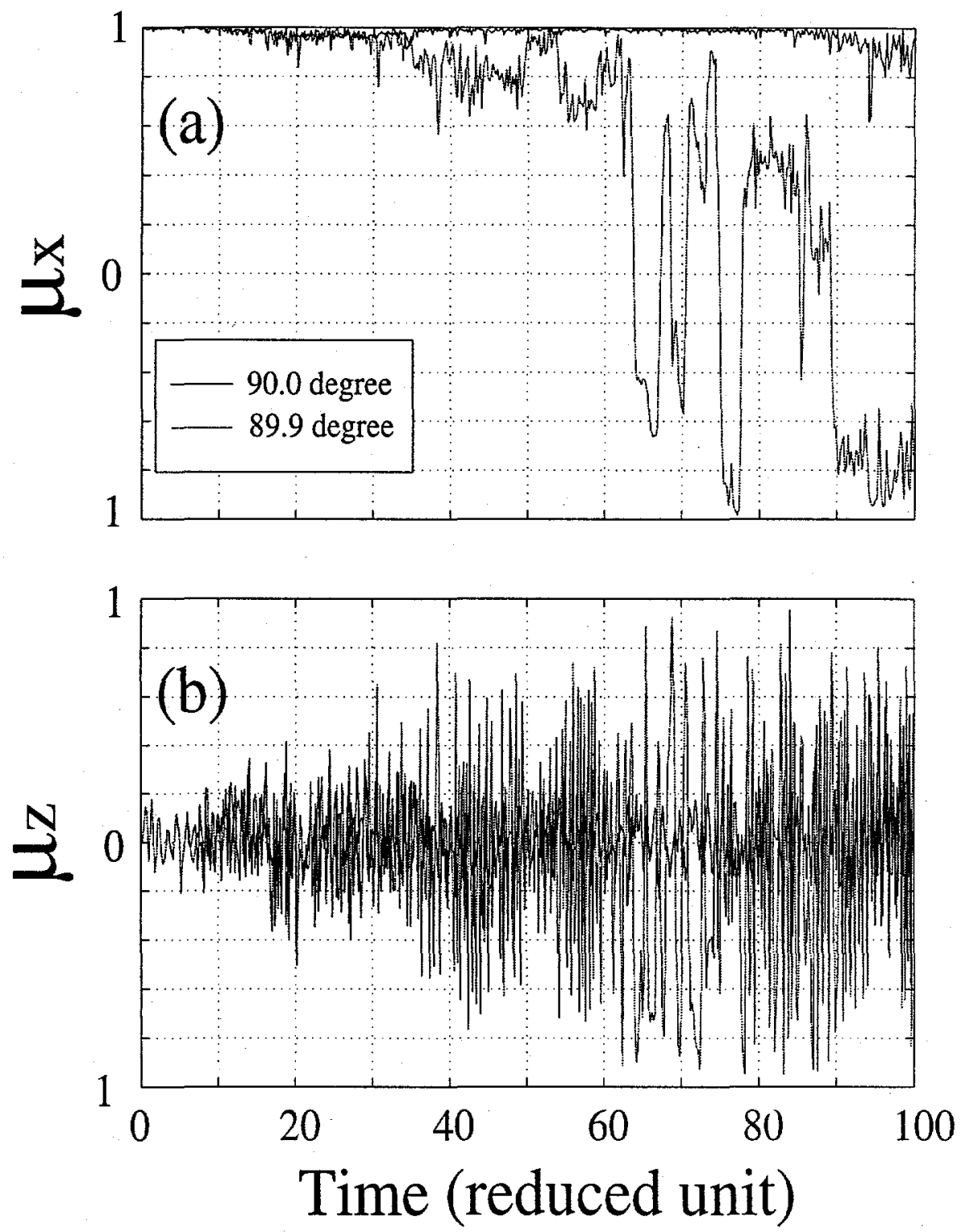

Figure 6.5: A typical trajectory of (a) $\mu_{x}(t)$ and (b) $\mu_{z}(t)$ of an arbitrarily tagged classical spin in a simple cubic lattice containing 125 equivalent classical spins with periodic boundary condition. All the spins are first treated by an ideal $89.9^{\circ}$ or $90.0^{\circ}$ pulse along $-y$ axis and then evolve under the truncated dipolar Hamiltonian in the rotating frame precessing with the Larmor frequency. The Ljapunov exponent is estimated to be 0.11 . 


\section{Bibliography}

[1] R. Kubo, J. Math. Phys. 4, 174 (1963).

[2] R. Kubo and N. Hashitsume, Prog. Theor. Phys. Suppl. 46, 210 (1970).

[3] V. Seshadri and K. Lindenberg, Physica A 115, 501 (1982).

[4] A. E. Stillman and J. H. Freed, J. Chem. Phys. 72, 550 (1980).

[5] B. Carmeli and D. Chandler, J. Chem. Phys. 89, 452 (1988).

[6] R. Graham and M. Hohnerbach, Z. Phys. Cond. Matt. 57, 233 (1984).

[7] R. F. Fox and J. Edison, Phys. Rev. 34, 482 (1986).

[8] P. I. Belobrov, G. M. Zaslavskii, and G. Kh. Tartakovskii, Sov. Phys. JETP 44, $945(1977)$

[9] P. W. Milonni, J. R. Ackerhalt, and H. W. Galbraith, Phys. Rev. Lett. 50, 966 (1983).

[10] J. R. Dormand and P. J. Prince, J. Comp. Appl. Math. 6, 19 (1980). 


\section{Chapter 7}

\section{Classical Spin Simulations}

\subsection{Abstract}

We have used Monte Carlo simulations, combined with microscopic Heisenberg equation of motion, to calculate accurately the many-spin FID waveform. The results are employed to verify the validity of our previous analysis in the limit of spin quantum number $I \rightarrow \infty$. It is shown that the spin self-trapped states could manifest themselves as small, yet persistent, components in the FIDs and may be the physical origin of the anomalous long-time behavior recently reported by Ding and McDowell. When the spin-lattice coupling strength and/or the dipolar order is increased, the resulting nonlinear polaronic/solitonic dynamics becomes even more pronounced. 


\subsection{Introduction}

Spin dynamics in quantum dissipative systems constitutes one of the most fundamental problems in the fields of magnetic resonance and condensed matter physics. A system with few quantal degrees of freedom (the "spin") is coupled to a fluctuating and dissipative bath ("lattice"). For abundant spins in condensed phases, particularly those with high gyromagnetic ratios, the homonuclear dipole-dipole interaction $\mathcal{H}_{d}$ is the dominant mechanism responsible for the spin-lattice coupling. When probing the spin dynamics, one first employs an external excitation which drives the whole system away from equilibrium and then measures its time-dependent response, the so-called free-induction decay (FID)). Rigorous theoretical predictions of the waveforms of the FID are complicated by the many-body nature of the Hamiltonian. Nevertheless, numerous approximate analytical theories have been proposed that can quantitatively or qualitatively describe the FID's short-time behavior: Gaussian decays for time smaller than the transverse relaxation time $\mathrm{T}_{2}[1]$; however, many essential features in the longer-time regimes still remain to be explored and verified, such as the beat structure [2] and the recently-reported anomalous long-time tails[3].

Advances in computer technology make spin-dynamics simulations very attractive. A genuine numerical approach to calculate the FID would require diagonalization of matrices of dimension $(2 I+1)^{N}$, where $I$ is the spin quantum number and $N$ is the number of coupled spins in the system. This would create a hopeless situation for $N$ sufficiently large to faithfully bring out the many-spin effects. In the classical limit, 
however,

$$
I \rightarrow \infty, \hbar \rightarrow 0, \gamma \hbar \sqrt{I(I+1)} \rightarrow|\mu|
$$

( $\gamma$ : gyromagnetic ratio, $\mu$ : magnetic moment) and the dimension greatly reduces to $2 N$ : two polar angles for each classical spin. Such computationally feasible classicalspin simulations can then be used to verify the validity of theoretical predictions in the limit of $I \rightarrow \infty$. One can also monitor a single trajectory; whereas in a real experiment one can only obtain an ensemble average over all trajectories.

For a system of $N$ identical classical spins in a rigid lattice subject to a large external Zeeman field $B_{\circ}$ along the $z$ axis, the effective interaction in the rotating frame precessing with the Larmor frequency $\omega_{0}=-\gamma B_{\circ}$ about the $z$ axis can be approximated by the truncated dipolar Hamiltonian $\mathcal{H}_{d}^{\circ}$

$$
\begin{gathered}
\mathcal{H}_{d}^{\circ}=\sum_{i=0}^{N-1} \sum_{j>i} \mathcal{H}_{d, i j}^{\circ}, \\
\mathcal{H}_{d, i j}^{\circ}=D_{i j}\left(2 \mu_{z i} \mu_{z j}-\mu_{x i} \mu_{x j}-\mu_{y i} \mu_{y j}\right), \\
D_{i j}=\frac{1-3 \cos ^{2} \theta_{i j}}{2 r_{i j}^{3}}
\end{gathered}
$$

where $r$ is the internuclear distance, and $\theta$ is the angle between the internuclear vector and the Zeeman field [2]. Since the Zeeman energy of a spin is around three orders of magnitude greater than its dipolar energy, high-field truncation is used so that only motions conserving the zeeman energy are allowed. Denote the interaction in the 
one-spin picture for a tagged spin labeled with subscript " 0 " as

$$
\mathcal{H}_{d}^{\circ}=-h_{0} \cdot \mu_{0}+\sum_{i=1}^{N-1} \sum_{j>i} \mathcal{H}_{d, i j}^{\circ}
$$

where $h_{0}=\left[h_{x 0}, h_{y 0}, h_{z 0}\right]$ are the dipolar local fields acting on the tagged spin produced by its neighbors, $\boldsymbol{\mu}_{0}=\left[\mu_{x 0}, \mu_{y 0}, \mu_{z 0}\right]$, and

$$
\begin{aligned}
& h_{z 0} \equiv-2 \sum_{i=1}^{N-1} D_{0 i} \mu_{z i} \\
& h_{\alpha 0} \equiv \sum_{i=1}^{N-1} D_{0 i} \mu_{\alpha i}(\alpha=x, y) .
\end{aligned}
$$

The classical equation of motion for any dynamical variable $A$ can be obtained by the following differential equation:

$$
\frac{d}{d t} A=\{A, H\}
$$

where $\{-,-\}$ indicates a Poisson bracket. The resulting dynamics is that each spin precesses under the torque exerted by its local fields,

$$
\frac{d}{d t} \mu_{i}(t)=\gamma \mu_{i}(t) \times h_{i}(t)(i=0, \cdots, N-1)
$$




\subsection{Computational Procedures}

The computational procedures are summarized as follows. Consider a simple-cubic lattice containing $N=5^{3}=125$ spins. Periodic boundary condition is applied to ensure that all dipoles have the same number of neighbors. We describe the spins in a coordinate system rotating about the Zeeman field with the Larmor frequency. Each spin sees in this system the dipolar field from its 32 nearest neighbors. Our restriction to the 32 nearest neighbors is immaterial in the FID's waveform, as dipolar coupling in condensed phases is a short-range interaction. The spin located at the center will be our observed spin, while the rest 124 spins will be regarded as the lattice. Based on the high temperature approximation $\left(k_{B} T \gg|\mu| B_{\mathrm{o}}\right)$, the initial density operator in the laboratory frame can be approximated as (Chapters 4 and 5)

$$
\rho(0) \propto|+\rangle_{z}\left\langle+\left.\right|_{z} \exp \left[-\beta \alpha \sum_{j=1}^{N-1} \mathcal{H}_{d, 0 j}^{\circ}\right)\right] \exp \left[-\beta\left(-B_{\circ} \sum_{i=1}^{N-1} \mu_{z i}+\sum_{i=1}^{N-1} \sum_{j>i} \mathcal{H}_{d, i j}^{\circ}\right)\right]
$$

where $\beta=1 / k_{B} T$ is the inverse temperature, $| \pm\rangle_{z}$ are the eigenstates of $I_{z}$ of the observed spin, and $\alpha$ is a parameter used to study the effect of dipolar order on the spin dynamics. For normal dipolar coupling, $\alpha$ is equal to 1 . Following the initial density operator, the tagged spin can be assumed to start from the initial condition $\boldsymbol{\mu}_{0}(0)=[0,0,|\boldsymbol{\mu}|]$, while the initial configuration for the lattice spins is obtained by generating $N$ uniformly distributed random vectors on the surface of a sphere with radius $|\boldsymbol{\mu}|[3]$. All the spins are first treated by an ideal pulse along $-y$ axis with 
tipping angle $\theta$. The corresponding rotation in the spin space is

$$
\mu_{i}\left(0_{+}\right)=\left[\begin{array}{ccc}
\cos (\theta) & 0 & -\sin (\theta) \\
0 & 1 & 0 \\
\sin (\theta) & 0 & \cos (\theta)
\end{array}\right] \boldsymbol{\mu}_{i}(0), i=0, \cdots, N-1
$$

where time $t=0_{+}$denotes the point immediately after the pulse. The time evolution of all the magnetic moments $\left\{\boldsymbol{\mu}_{i}(t)\right\}_{(i=0, \cdots, N-1)}$ is then numerically solved by integrating the system of differential equations, Eq (7.9), based on explicit Runge-Kutta $(4,5)$ algorithm of Dormand-Prince [4]. Finally, the FID is estimated by averaging $\mu_{x 0}(t)$ over the trajectories weighed by the equilibrium Boltzman probabilities of the initial configurations

$$
\left\langle\mu_{x 0}(t)\right\rangle \propto \sum_{\text {trajectories }} \rho(0) \mu_{x 0}(t)
$$

For a given set of initial configurations and calculated trajectories, one can use the symmetry of the equations of motion to generate additional sets of inital configurations whose trajectories are related by symmetry to the calculated trajectories(Table 1). This increases the number of initial configurations sampled, thereby reducing the error due to finite Monte Carlo sampling, without having to due any additional trajectory calculations. For systems consisting of just one type of particle, it is sensible 
Table 7.1: New Trajectories Based on Symmetry Considerations $(i=0, \cdots, N-1)$

\begin{tabular}{|c|c|}
\hline Initial Configurations & Trajectories \\
\hline & \\
$\left\{\mu_{x i}\left(0_{+}\right), \mu_{y i}\left(0_{+}\right), \mu_{z i}\left(0_{+}\right)\right\}$ & $\left\{\mu_{x i}(t), \mu_{y i}(t), \mu_{z i}(t)\right\}$ \\
$\left\{-\mu_{x i}\left(0_{+}\right),-\mu_{y i}\left(0_{+}\right), \mu_{z i}\left(0_{+}\right)\right\}$ & $\left\{-\mu_{x i}(t),-\mu_{y i}(t), \mu_{z i}(t)\right\}$ \\
$\left\{-\mu_{x i}\left(0_{+}\right), \mu_{y i}\left(0_{+}\right),-\mu_{z i}\left(0_{+}\right)\right\}$ & $\left\{-\mu_{x i}(t), \mu_{y i}(t),-\mu_{z i}(t)\right\}$ \\
$\left\{\mu_{x i}\left(0_{+}\right),-\mu_{y i}\left(0_{+}\right),-\mu_{z i}\left(0_{+}\right)\right\}$ & $\left\{\mu_{x i}(t),-\mu_{y i}(t),-\mu_{z i}(t)\right\}$ \\
\hline
\end{tabular}

to use the reduced units. In this chapter, the time $t$ is expressed in the unit of

$$
\frac{2 r_{\circ}^{3}}{3 \gamma|\mu|}
$$

where $r_{\mathrm{o}}$ is the lattice constant.

\subsection{Simulation Results}

Figure 7.1 shows the FID of the tagged spin based on 40,000 trajectories with dipolar-order parameter $\alpha=1$. The other physical parameters used in the chapter are: $k_{B} T=200, B_{\circ}=10$. In conventional NMR theory, the transverse magnetization is completely dephased within tens of microseconds due to the observed spins evolving under different local fields. With the assumption that the spin does not affect the evolution of the lattice, one obtains the typical NMR result, as shown in Fig. 7.1b. However, when one takes into account the effect of the spin on the evolution of the lattice, that is, takes into account the reaction field, one obtains a small, yet persistent 
component buried in the FID [ Fig. 7.1a].

Such long time tail becomes even more obvious when one increases the coupling strength of the tagged spin to the lattice, as shown in Fig. 7.2 (dipolar-order parameter $\alpha=1$ ). The oscillatory beat structure in the FIDs is the manifestation of the cooperative coherent dipole motions [7]. In Fig. 7.2b, the oscillation of the FID is center at $\left\langle\mu_{x 0}(t)\right\rangle=0$. This is consistent with the prediction made by Kubo and Toyabe, who treats the local field at a given spin as a stochastic process independent of that spin [8]. As discussed in Chapter 6, this appealing theory conflicts with the proper attainment of a canonical equilibrium due to the fact that the influence of the reaction field is disregarded. On the contrary, when the reaction field is turned on, as shown in Fig. 7.2a, the FID oscillates about a new equilibrium state with $\left\langle\mu_{x 0}(t)\right\rangle \neq 0$. It verifies the physical picture portrayed in the previous chapters based on the spinboson Hamiltonian: solitonic modes originated from the nonlinearity triggered by the joint action of the lattice fluctuations and the reaction field. This is even more clear when one looks at the individual trajectories shown in Fig. 7.3. Certain events exhibit states where the spin evolves into a trapped state due to the reaction field, i.e., the local fields and spin evolve to align, which causes the spin to be trapped, since $\mu_{0}(t) \times h_{0}(t)=0$. Random fluctuations of the other lattice spins eventually cause the local fields to become misaligned with the tagged spin, thus causing the spin to be no longer trapped.

It is also interesting to observe the effect of the dipolar order in the initial density 

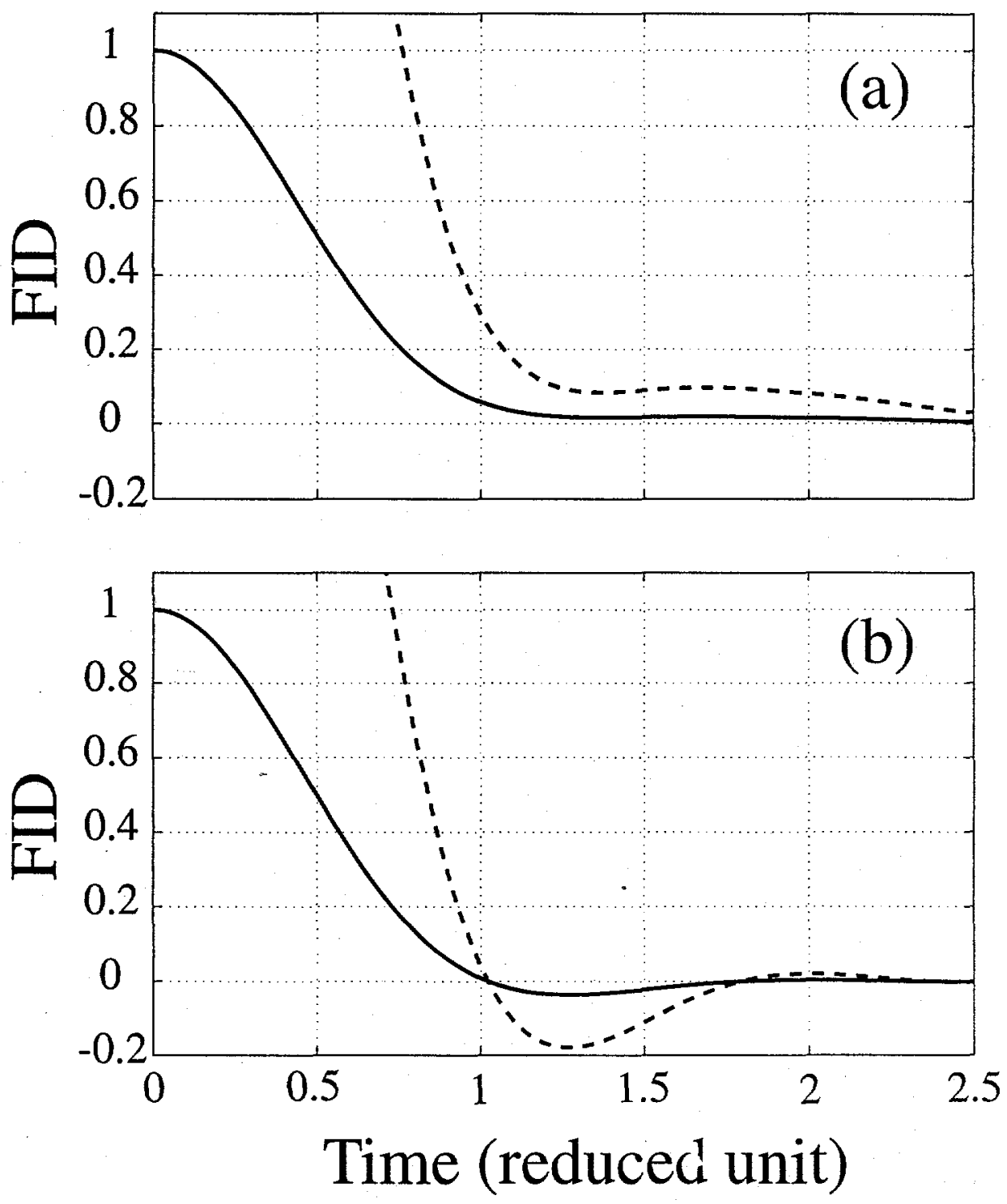

Figure 7.1: The FID of a tagged spin in a simple cubic lattice of spins (a) with reaction field and (b) without. The dashed line represents a magnification by a factor of 5. Figure (b) corresponds to the typical free induction decay, which goes to zero for long times; on the other hand, figure (a) shows a nonvanishing component. 

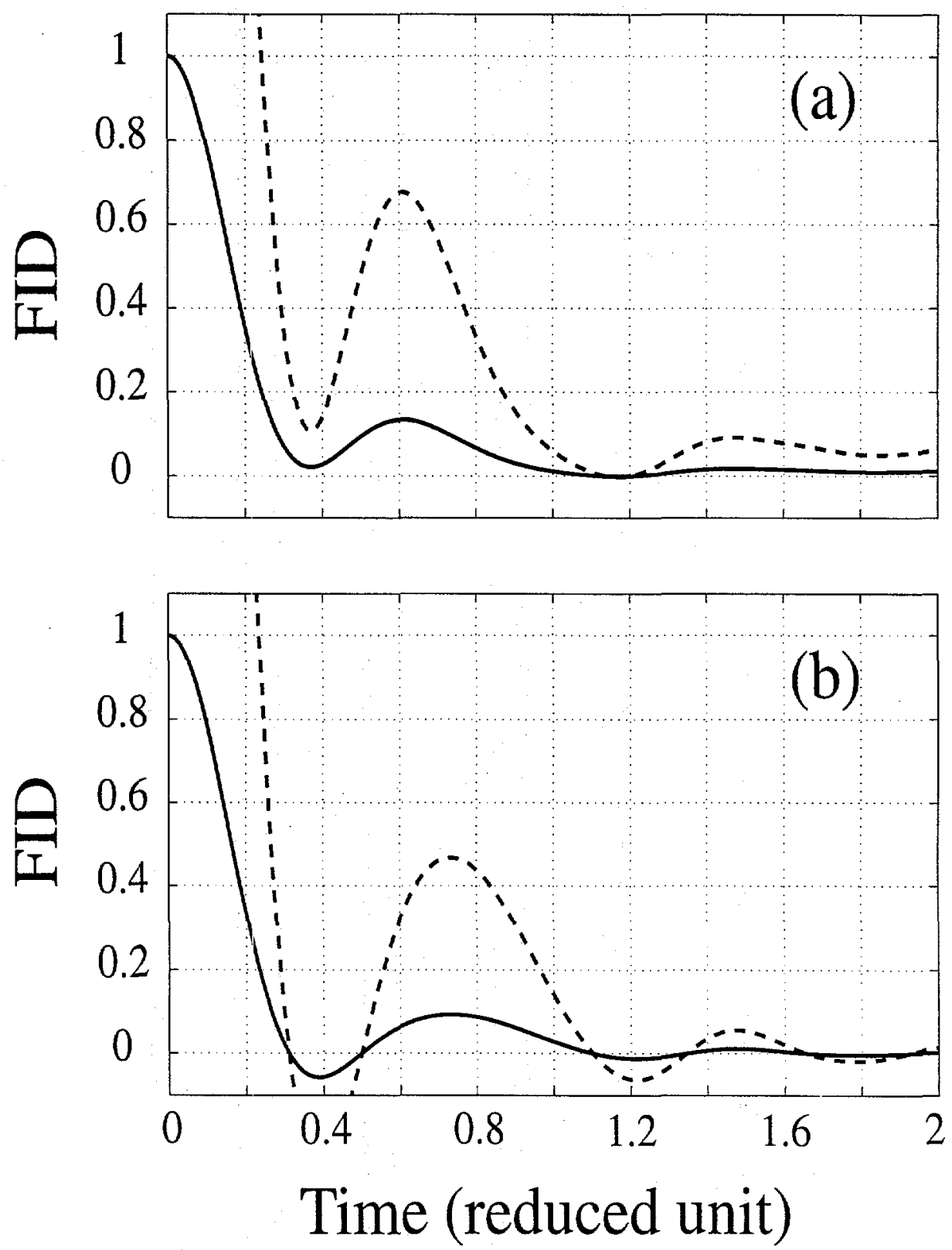

Figure 7.2: The FID of a tagged spin coupled to a simple-cubic lattice of spins (a) with and (b) wihtout reaction field. The dashed line represents a magnification of a factor of 5 . The coupling constants of the tagged spin to the lattice spins have been increased by a factor of 3 relative to Fig. 7.1's coupling constants. In figure (a), one sees a larger amplitude of the long time tail relative to Fig. 7.1a. 

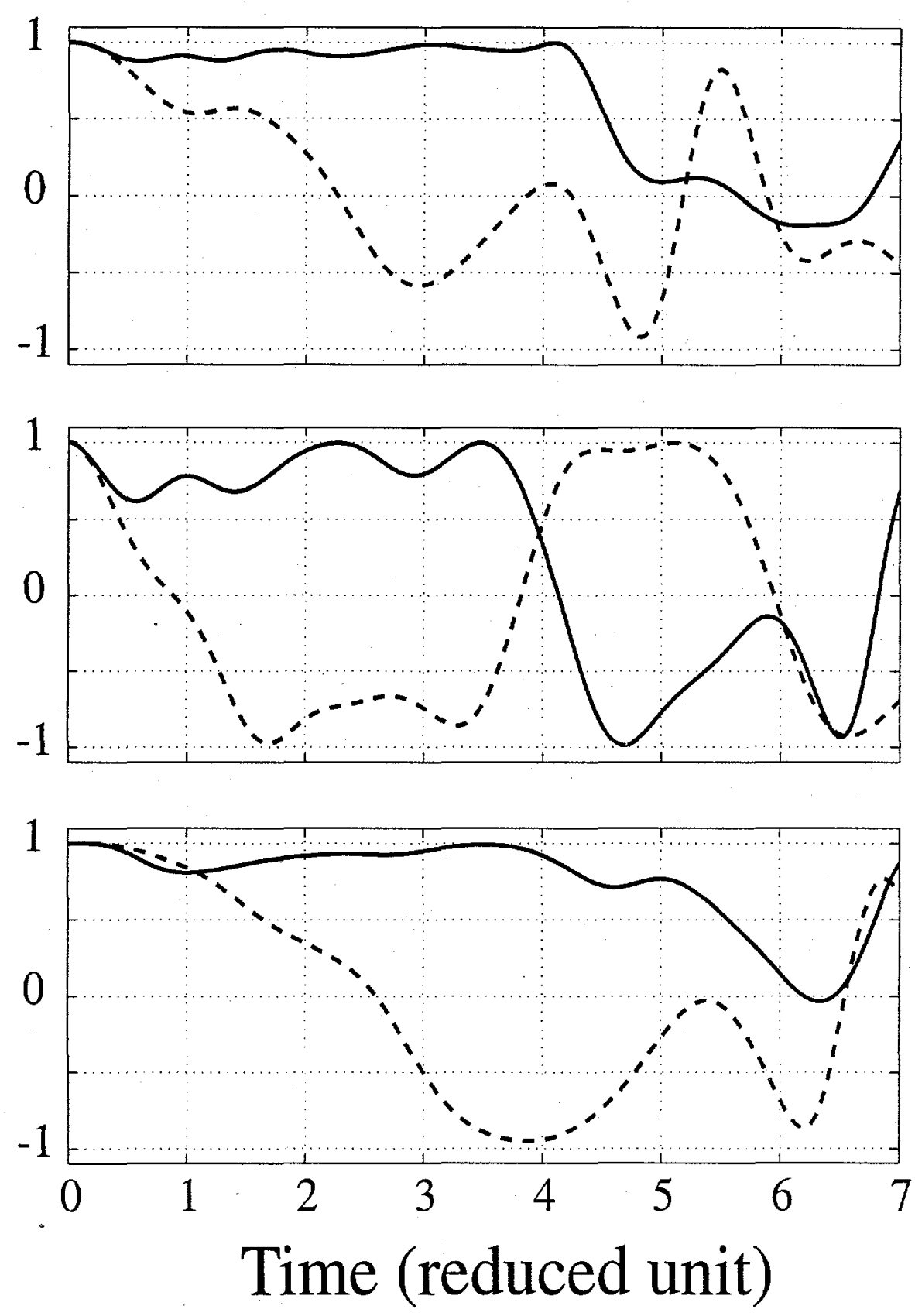

Figure 7.3: Trajectories of the tagged spin in a simple-cubic lattice. The solid lines exhibit self trapping, due to the reaction field, whereas the dashed trajectories, which do not include the reaction field, do not evolve into trapped states. 
operator towards the development of the solitonic modes. This is shown in Fig. 7.4 for dipolar-order parameter $\alpha=30$ (solid line) and $\alpha=15$ (dash line). It reveals the possibility of enhancing the long-time tails by converting zeeman order into dipolar order. If this observation is further confirmed by quantum spin simulations and experiments, then, without resorting to demanding decoupling techniques in the spin and/or spatial spaces, the phenomena of spin self-trapping under dipolar local fields may provide solid-state NMR a convenient way to achieve better resolution. One can use a sufficiently long delay between simple pulse preparation and data acquisition to filter out the initial fast-decaying part before performing Fourier transformation. 

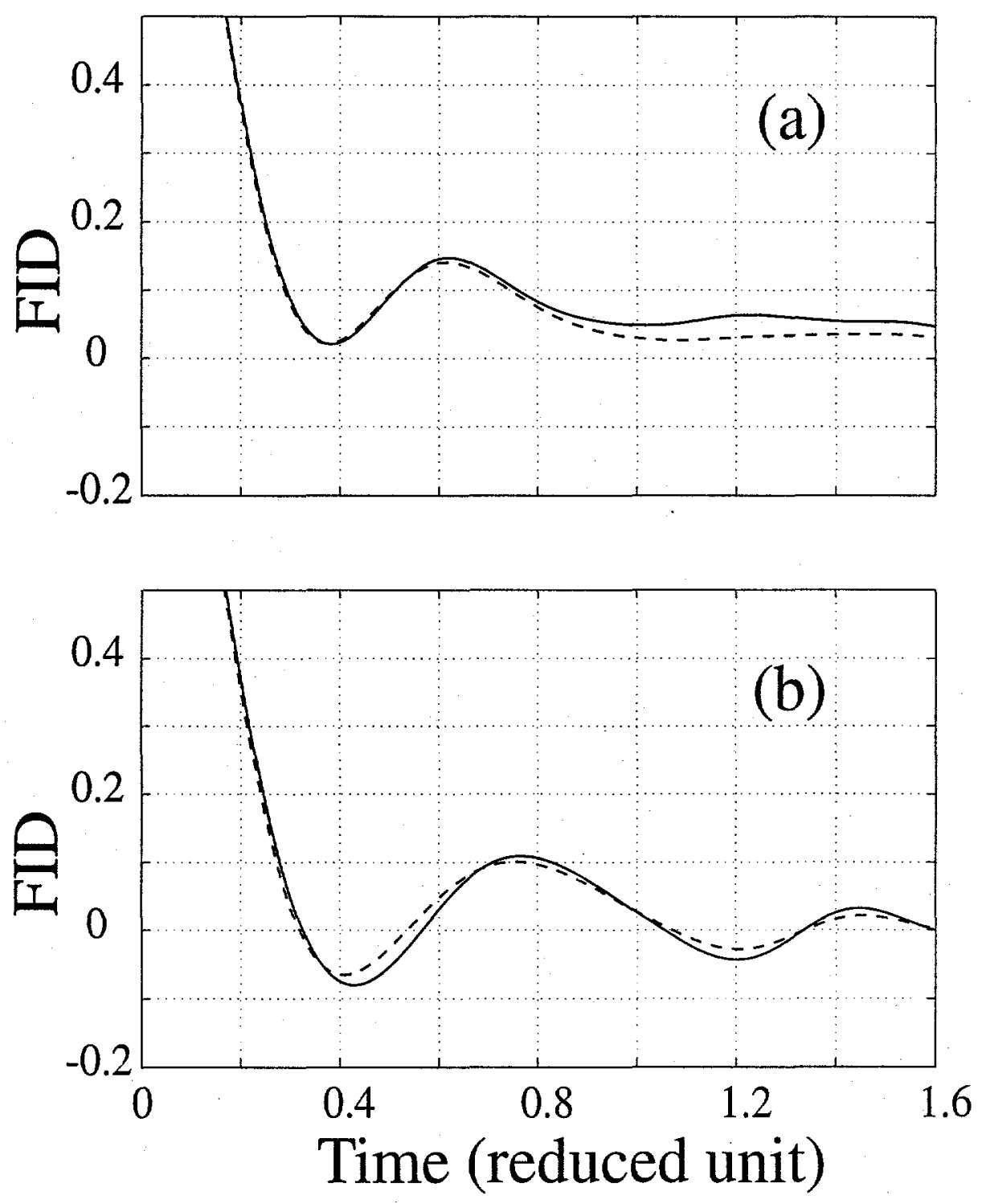

Figure 7.4: The FID of a tagged spin coupled to a simple-cubic lattice of spins (a) with and (b) wihtout reaction field. The dipolar-order parameter $\alpha$ equals to 30 (solid line) and 15 (dash line). 


\section{Bibliography}

[1] See, e.g., E. T. Cheng and J. D. Memory, Phys. Rev. B 6, 1714 (1972); R. Kubo and N. Hashitsume, Prog. Theor. Phys. Suppl. 46, 210 (1970); P. W. Anderson and P. R. Weiss, Rev. Mod. Phys. 25, 269 (1953).

[2] I. J. Lowe and R. E. Norberg, Phys. Rev. 107, 46 (1957).

[3] S. Ding and C. A. McDowell, J. Magn. Reson. A 111, 212 (1994); ibid. 115, 141 (1995); ibid. 117, 171 (1995); ibid. 120, 261 (1996); S. Ding and C. A. McDowell, Chem. Phys. Lett. 255, 151 (1996); ibid. 259, 538 (1996).

[4] A. Abragam, Principles of Nuclear Magnetism (Clarendon, Oxford, 1961).

[5] G. Marsaglia, Ann. Math. Stat. 43, 645 (1972).

[6] J. R. Dormand and P. J. Prince, J. Comp. Appl. Math. 6, 19 (1980).

[7] A. V. Ivanova and B. N. Provotorov, Chem. Phys. Reports. 14, 1127 (1995).

[8] R. Kubo and T. Toyabe, in Magnetic Resonance and Relaxation, edited by R. Blinc (North Holland, Amsterdam, 1967), p. 810. 
Part III

Appendix 



\section{Appendix A}

\section{Advanced NMR Signal Processing}

\section{Package}

Within the last decade, there has been a flurry of research activity into formulating and comparing alternative means of NMR signal processing $[1,2,3,4,5,6,7,8,9$, 10]. The primary motivation has apparently been the development of techniques for accurate spectral quantification and enhanced spectral resolution. One of the most effective ways to gain a deep appreciation and understanding of such advanced techniques is to process NMR signals. There is a great deal of information to be gained by experimenting with algorithms, testing them on real FIDs, developing new approaches, and discovering at what point the theory begins to break down in practice. Therefore, to assist in the implementation of these advanced NMR signal processing techniques, a package of MATLAB programs is constructed, as summarized 
in Table A.1. MATLAB [11] has the advantage of providing easy access to matrix software developed by the LINPACK [12] and EISPACK [13] projects, and allowing rapid code development and refinement [14]. None of these programs in this package are particularly long and may be typed-in by hand without too much difficulty.

\section{A.1 Matrix Pencil}

Assume the FID can be decomposed into a set of exponentially damped sinusoids,

$$
\begin{array}{r}
y_{n}=x_{n}+w_{n}=\sum_{i=1}^{M}\left|a_{i}\right| \exp \left(j \theta_{i}\right) \exp \left[\left(-\alpha_{i}+j 2 \pi f_{i}\right) n\right]+w_{n} \\
=\sum_{i=1}^{M} a_{i} z_{i}{ }^{n}+w_{n} \quad n=0,1, \ldots, N-1
\end{array}
$$

where $\left|a_{i}\right|, \alpha_{i}, f_{i}, \theta_{i}$ represent the absolute amplitüdes, damping factors (inverse time constants), frequencies, and phases of the $M$ distinct exponentials, respectively; $j$ is used to denote $\sqrt{-1} . z_{i} \equiv \exp \left(-\alpha_{i}+j 2 \pi f_{i}\right)$ is the "signal pole" and $a_{i} \equiv\left|a_{i}\right| \exp \left(j \theta_{i}\right)$ is the "complex amplitude". There is an important difference between the complex amplitude $a_{i}$ and the signal pole $z_{i}$ in the functional form of Eq. (A.2); the signal model is linear in the first and nonlinear in the second. The complications of nonlinearity can be circumvented by invoking the linear prediction principle or the matrix pencil method. 
Table A.1: Summary of Advanced NMR Signal Processing Package

\begin{tabular}{|l|l|l|}
\hline Section & Program & Description \\
\hline Matrix Pencil & $\begin{array}{l}\text { mp_mp.m } \\
\text { mp_p.m }\end{array}$ & $\begin{array}{l}\text { matrix pencil } \\
\text { linear prediction }\end{array}$ \\
\hline Set Theoretic Estimation & $\begin{array}{l}\text { ste_moor.m } \\
\text { ste_rank.m } \\
\text { ste_eigen } \\
\text { ste_toep.m } \\
\text { ste_herm.m } \\
\text { ste_sub.m }\end{array}$ & $\begin{array}{l}\text { B. de Moor algorithm } \\
\text { matrix rank mapping } \\
\text { eigenstructure mapping } \\
\text { Toeplitz structure mapping } \\
\text { Hermitian structure mapping } \\
\text { subsequence property mapping }\end{array}$ \\
\hline System Identification & $\begin{array}{l}\text { si_pade.m } \\
\text { si_prony.m } \\
\text { si_ter.m } \\
\text { si_auto.m } \\
\text { si_cov.m }\end{array}$ & $\begin{array}{l}\text { Padé approximation } \\
\text { Prony method } \\
\text { iterative prefiltering method } \\
\text { autocorrelation method } \\
\text { covariance method }\end{array}$ \\
\hline Spectral Estimation & $\begin{array}{l}\text { se_mlm.m } \\
\text { se_mem.m } \\
\text { se_phd.m } \\
\text { se_music.m } \\
\text { se_ev.m } \\
\text { se_minnorm.m } \\
\text { se_principal.m }\end{array}$ & $\begin{array}{l}\text { MigsIC method } \\
\text { eigenvector method } \\
\text { minimum norm method } \\
\text { principal component method } \\
\text { maximum entropy method } \\
\text { misarenko harmonic decomposition }\end{array}$ \\
\hline
\end{tabular}




\section{A.1.1 Matrix Pencil Method with Information Theoretic Cri-}

\section{teria}

The matrix pencil method involves finding the signal poles, $z_{i}$, directly by solving a generalized eigenvalue problem. Let $\boldsymbol{X}_{0}$ and $\boldsymbol{X}_{1}$ be two noise-free data matrices with dimension $(N-L) \times L$ defined by

$$
\boldsymbol{X}_{0}=\left[\begin{array}{cccc}
x_{L-1} & x_{L-2} & \cdots & x_{0} \\
x_{L} & x_{L-1} & \cdots & x_{1} \\
\vdots & \vdots & \ddots & \vdots \\
x_{N-2} & x_{N-3} & \cdots & x_{N-L-1}
\end{array}\right], \boldsymbol{X}_{1}=\left[\begin{array}{cccc}
x_{L} & x_{L-1} & \cdots & x_{1} \\
x_{L+1} & x_{L} & \cdots & x_{2} \\
\vdots & \vdots & \ddots & \vdots \\
x_{N-1} & x_{N-2} & \cdots & x_{N-L}
\end{array}\right]
$$

where $L$ is called the pencil parameter. The the signal poles $z_{i}$ can be identified with the $M$ non-zero generalized eigenvalues of the matrix pair $\left(\boldsymbol{X}_{1}, \boldsymbol{X}_{0}\right)$,

$$
\boldsymbol{X}_{1} \boldsymbol{q}_{i}=z_{i} \boldsymbol{X}_{0} \boldsymbol{q}_{i}
$$

where $\boldsymbol{q}_{i}$ is the eigenvector associated with the eigenvalue (and signal pole) $z_{i}$.

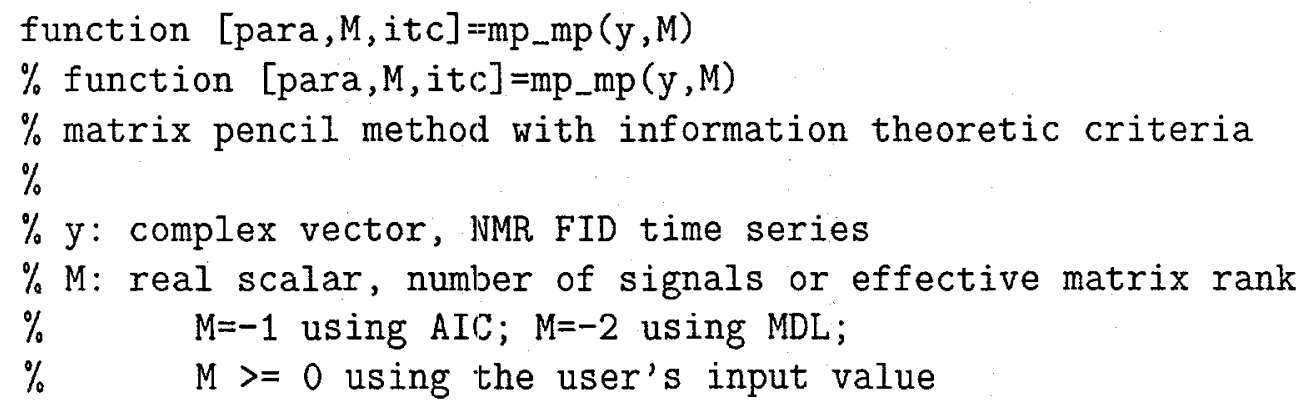


$\%$ para: real $M * 4$ matrix, estimated damping factor,

$\% \quad$ frequency, amplitude, phase

$\%$ itc: real vector, containing AIC or MDL function values

$\% * * * * * * * * * *$ begin of function mp_mp.m $* * * * * * * * * *$

$\mathrm{y}=\mathrm{y}(:)$;

$\mathrm{N}=$ length $(\mathrm{y})$;

$\%$-- pencil parameter

$\mathrm{L}=\mathrm{fl} \operatorname{loor}(\mathrm{N} / 3)$;

$\%--Y 0=Y(:, 2: L+1), Y 1=Y(:, 1: L)$

$Y=$ toeplitz $(y(L+1: N), y(L+1:-1: 1))$;

$\%$--- singular value decomposition

$[\mathrm{U}, \mathrm{S}, \mathrm{V}]=\operatorname{svd}(\mathrm{Y}(:, 2: \mathrm{L}+1), 0)$;

$\mathrm{S}=\operatorname{diag}(\mathrm{S})$;

itc=zeros $(1, \mathrm{~L})$;

$\%$--- determining $M$ by AIC

if $M==-1$

for $\mathrm{k}=0: \mathrm{L}-1$;

itc $(k+1)=-2 * N * \operatorname{sum}(\log (S(k+1: L))) \quad \ldots$

$+2 * \mathrm{~N} *(\mathrm{~L}-\mathrm{k}) * \log ((\operatorname{sum}(\mathrm{S}(\mathrm{k}+1: \mathrm{L})) /(\mathrm{L}-\mathrm{k})))+2 * \mathrm{k} *(2 * \mathrm{~L}-\mathrm{k})$;

end

[tempY, tempI]=min (itc); M=tempI-1;

end

$\%$--- determining $M$ by $M D L$

if $\mathrm{M}==-2$

for $\mathrm{k}=0: \mathrm{L}-1$;

$\operatorname{itc}(k+1)=-N * \operatorname{sum}(\log (S(k+1: L))) \ldots$

$+\mathrm{N} *(\mathrm{~L}-\mathrm{k}) * \log ((\operatorname{sum}(\mathrm{S}(\mathrm{k}+1: \mathrm{L})) /(\mathrm{L}-\mathrm{k})))+\mathrm{k} *(2 * \mathrm{~L}-\mathrm{k}) * \log (\mathrm{N}) / 2$;

end

[tempY, tempI] $=\min ($ itc) ;

$M=$ tempI -1 ;

end

$\%$--- signal pole $z=\exp (\mathrm{s})$

$s=\log (\operatorname{eig}(\operatorname{diag}(1 . / S(1: M)) * \ldots$

$((U(:, 1: M) ' * Y(:, 1: L)) * V(:, 1: M))))$;

$\mathrm{Z}=\operatorname{zeros}(\mathrm{N}, \mathrm{M})$;

for $\mathrm{k}=1: M$;

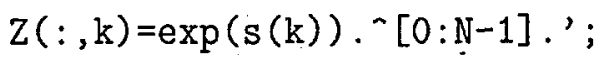

end;

$\%$--- Iinear least squares analysis

$\mathrm{a}=\mathrm{Z} \backslash \mathrm{y}$;

$\operatorname{para}=[-\operatorname{real}(\mathrm{s}) \operatorname{imag}(\mathrm{s}) / 2 / \mathrm{pi} \operatorname{abs}(\mathrm{a}) \operatorname{imag}(\log (\mathrm{a} . / \mathrm{abs}(\mathrm{a})))]$;

return 
$\%$

end of function mp_mp.m

\section{A.1.2 Linear Prediction with Singular Value Decomposition}

The principles of linear prediction with singular value decomposition (LPSVD) have been extensively documented. In summary, one first solves the following linear prediction equations for the coefficients $\left\{c_{i}\right\}_{1 \leq i \leq L}$ of the prediction polynomial,

$$
\left[\begin{array}{cccc}
y_{L-1} & y_{L-2} & \cdots & y_{0} \\
y_{L} & y_{L-1} & \cdots & y_{1} \\
\vdots & \vdots & \ddots & \vdots \\
y_{N-2} & y_{N-3} & \cdots & y_{N-L-1}
\end{array}\right]\left[\begin{array}{c}
c_{1} \\
c_{2} \\
\vdots \\
c_{L}
\end{array}\right]=\left[\begin{array}{c}
y_{L} \\
y_{L+1} \\
\vdots \\
y_{N-1}
\end{array}\right] .
$$

The roots of the prediction polynomial $P(z)=1-\sum_{i=1, L} c_{i} z^{-i}$ then produce the $M$ signal poles $\left\{z_{i}\right\}_{1 \leq i \leq M}$. The success of LPSVD resides in the ability of linear prediction to extend truncated FIDs for resolution enhancement, or to estimate missing or corrupted initial data points for baseline and phase corrections. The corrupting effects of noise can be mitigated through a judicious combination of an over-cstimated prediction order ( $L \gg M$, introducing extra $L-M$ noise-related poles) to account for the noise in the measurements, and SVD-based signal-subspace techniques to discriminate between signal and noise.

function para=mp_lp $(y, M)$

$\%$ function para=mp_lp $(y, M)$ 
$\%$ linear prediction with singular value decomposition

$\%$ reference:

$\%$ R. Kumaresan, D. W. Tufts IEEE Trans. Acoust. Speech Signal Processing

$\%$ vol. ASSP-30, 837-840, 1982.

$\%$ arguments :

$\% y$ : complex vector, NMR FID time series

$\% \mathrm{M}$ : real scalar, number of signals or effective matrix rank

$\%$ para: real $M * 4$ matrix, estimated damping factor,

$\% \quad$ frequency, amplitude, phase

$\% * * * * * * * * * *$ begin of function mp_lp.m $* * * * * * * * * *$

$\mathrm{y}=\mathrm{y}(:) ;$

$\%$--- number of complex data points in FID

$\mathrm{N}=$ length $(\mathrm{y})$;

$\%$--- linear prediction order $L=3 / 4 * \mathrm{~N}$

$\mathrm{L}=$ floor $(\mathrm{N} * 3 / 4)$;

$\%$--- backward prediction data matrix

$A=\operatorname{hankel}(\operatorname{conj}(y(2: N-L+1))$, $\operatorname{conj}(y(N-L+1: N)))$;

$\%$--- backward prediction data vector

$\mathrm{h}=\operatorname{conj}(\mathrm{y}(1: \mathrm{N}-\mathrm{L}))$;

$\%$--- singular value decomposition

$[\mathrm{U}, \mathrm{S}, \mathrm{V}]=\mathrm{svd}(\mathrm{A}, 0)$;

clear A;

$\mathrm{S}=\operatorname{diag}(\mathrm{S})$;

$\%$--- bias compensation

bias $=\operatorname{mean}(\mathrm{S}(\mathrm{M}+1: \min ([\mathrm{N}-\mathrm{L}, \mathrm{L}])))$;

$\%$--- prediction polynomial coefficients

$\left.\mathrm{b}=-\mathrm{V}(:, 1: \mathrm{M}) *\left(\operatorname{diag}(1 . /(\mathrm{S}(1: \mathrm{M})-\mathrm{bias})) *(\mathrm{U}(:, 1: \mathrm{M}))^{\prime} * \mathrm{~h}\right)\right)$;

$\%$--- polynomial rooting

$s=\operatorname{conj}(\log (\operatorname{roots}([b($ length $(b):-1: 1) ; 1])))$;

$\%$--- extract true signal poles

$\mathrm{s}=\mathrm{s}(\mathrm{find}(\mathrm{real}(\mathrm{s})<0))$;

$\mathrm{Z}=\mathrm{zeros}(\mathrm{N}$, length $(\mathrm{s}))$;

for $\mathrm{k}=1$ : length $(\mathrm{s})$;

$\mathrm{Z}(:, \mathrm{k})=\exp (\mathrm{s}(\mathrm{k})) \cdot{ }^{\wedge}[0: \mathrm{N}-1] .^{\prime} ;$

end;

$\%$--- linear least squares analysis

$\mathrm{a}=\mathrm{Z} \backslash \mathrm{y}$;

$\operatorname{para}=[-\operatorname{real}(\mathrm{s}) \operatorname{imag}(\mathrm{s}) / 2 / \mathrm{pi}$ abs (a) $\operatorname{imag}(\log (\mathrm{a} . / \mathrm{abs}(\mathrm{a})))]$;

return

$\% * * * * * * * * * *$ end of function mp_lp.m $* * * * * * * * * *$ 


\section{A.2 Set Theoretic Estimation}

If $\left(\Psi_{i}\right)_{1 \leq i \leq I}$ is the collection of $I$ propositions representing a priori knowledge on the observed NMR signal and $\Xi$ is the solution space, a collection of so-called property sets $\left(S_{i}\right)_{1 \leq i \leq I}$ can be constructed in a propositional manner, namely,

$$
S_{i}=\left\{a \in \Xi \mid \Psi_{i} \text { holds for } a\right\}
$$

A set theoretic estimate is any object consistent with all available information, i.e., any point in the set intersection

$$
S=\bigcap_{i=1}^{I} S_{i}=\left\{a \in \Xi \mid \forall i \in\{1, \cdots, I\} \Psi_{i} \text { holds for } a\right\}
$$

A signal-enhanced FID, $y$, can be formally obtained by solving the following best feasible approximation problem

$$
\inf _{\boldsymbol{y} \in S} d(\boldsymbol{x}, \boldsymbol{y})
$$

where "inf" denotes the greatest lower bound operator, $\boldsymbol{x}$ is the measured noisy FID, and " $d(\boldsymbol{x}, \boldsymbol{y})$ " refers to a distance measure.

In the following, the algorithm proposed by B. de Moor $[19,20]$ is first adopted to solve the resulting best feasible approximation problem Eq. (A.8). Then, a more general and efficient algorithm, iterative parallel projections, is presented. 


\section{A.2.1 B. de Moor algorithm}

If the spin relaxation can be treated as a first order process, the measured NMIR FID will be a sum of exponentially damped sinusoids perturbed by additive noise. Consider the data matrix $\boldsymbol{X}$ formed from such a FID $\boldsymbol{x}=\left[x_{0}, x_{1}, \ldots, x_{N-1}\right]^{T}$,

$$
\boldsymbol{X}(\boldsymbol{x})=\left[\begin{array}{cccc}
x_{0} & x_{1} & \cdots & x_{N-L} \\
x_{1} & x_{2} & \cdots & x_{N-L+1} \\
\vdots & \vdots & \ddots & \vdots \\
x_{L} & x_{L+1} & \cdots & x_{N-1}
\end{array}\right]
$$

where "T" denotes matrix transpose and $L$ is a parameter chosen by the user. It follows that $\boldsymbol{X}$, when constructed from noiseless FID, possesses two important properties, (i) $\Psi_{1}$, Hankel structure, and (ii) $\Psi_{2}$, rank deficiency. A Hankel matrix is one which is symmetrical about any cross-diagonal, while the rank deficiency of $\boldsymbol{X}$ results from $x$ being the impulse response of a finite dimensional linear system of relatively low order.

With above a priori knowledge, the general expression of the best feasible approximation problem can be specified as

$$
\inf \boldsymbol{y \in S = S _ { 1 } \cap S _ { 2 }} d(\boldsymbol{x}, \boldsymbol{y})
$$

where property sets $S_{1}=\{\boldsymbol{x} \in \Xi \mid \boldsymbol{X}(\boldsymbol{x})$ is a Hankel matrix $\}, S_{2}=\{\boldsymbol{x} \in \Xi \mid \operatorname{rank}[\boldsymbol{X}(\boldsymbol{x})]=$ 
$M\}$, and $\boldsymbol{X}(\boldsymbol{x}) \in C^{(L+1) \times(N-L+1)}$. The specific projection operator required to solve Eq. (A.10) has recently been developed in the context of constrained total least squares $[15,16,17,18]$. The algorithm proposed by B. de Moor $[19,20]$ is adopted here, and its MATLAB implementation is listed below. Much work needs to be done on possible refinements, accelerations, and memory-thrift.

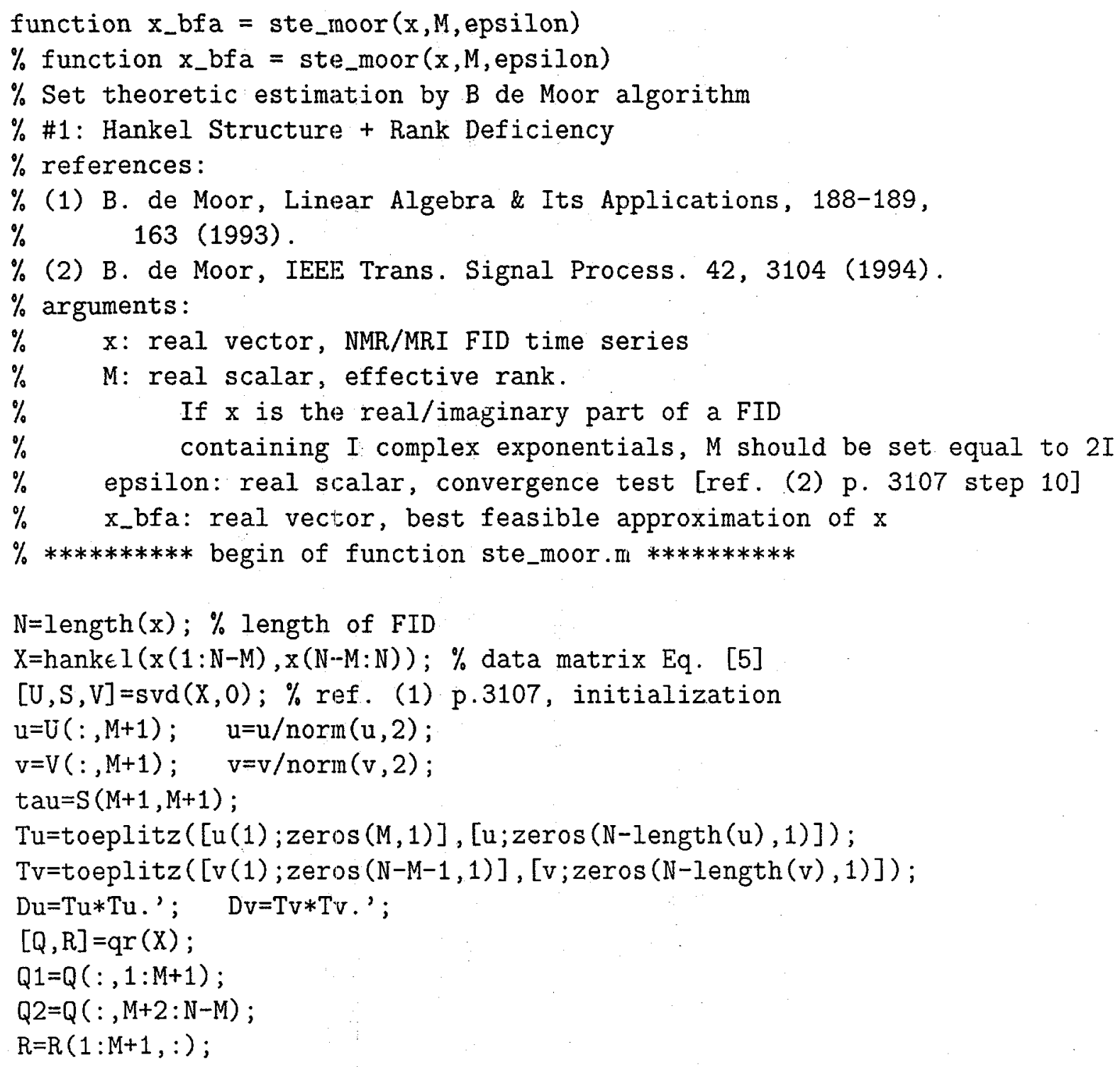




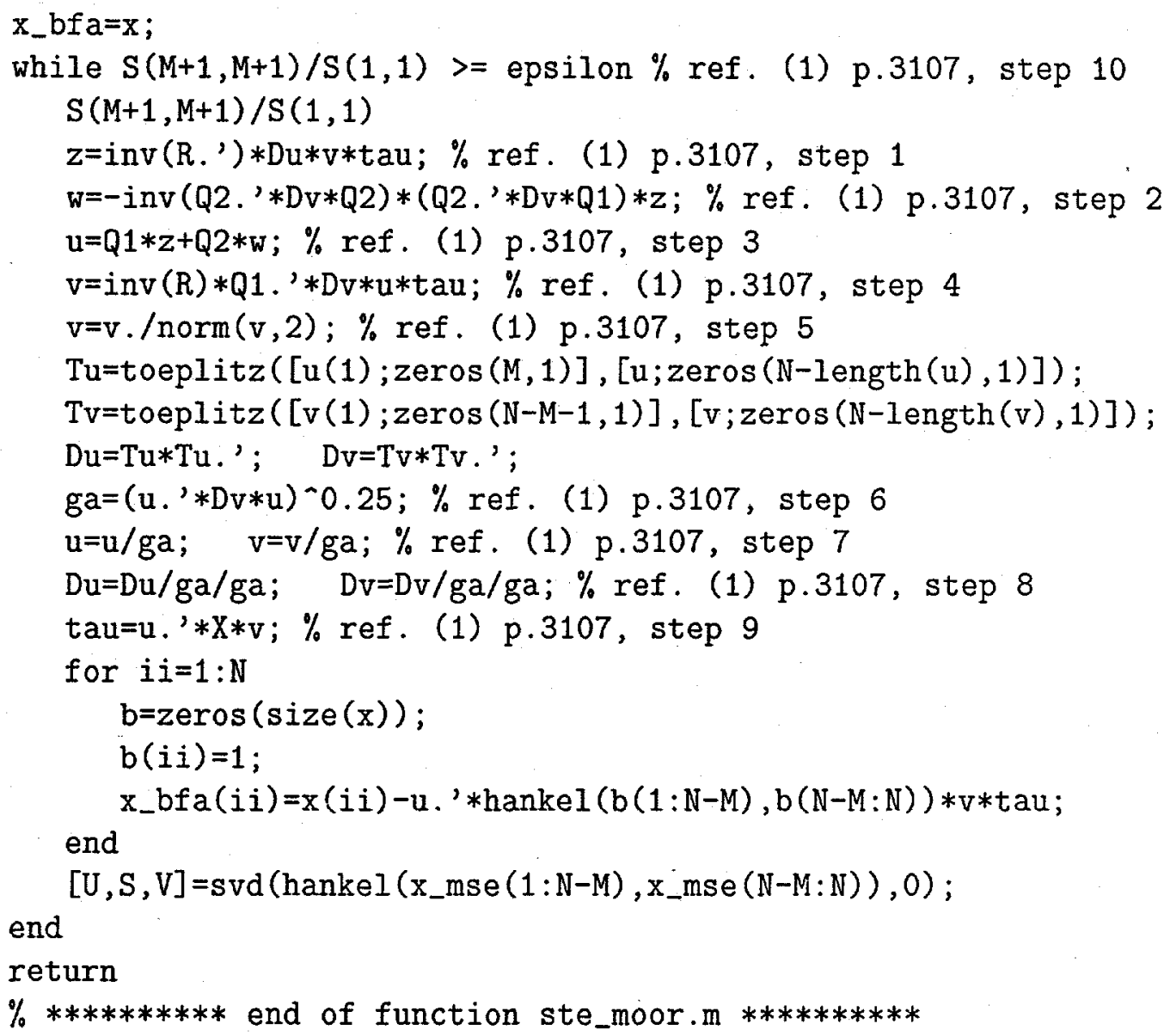

\section{A.2.2 Iterative Parallel Projections}

The algorithm of iterative parallel projections, instead of attacking Eq. (A.8) directly, exploits the techniques of best approximation and nonlinear programming to solve the potentially more tractable set of problems

$$
\inf _{\boldsymbol{y} \in S_{k}} d(\boldsymbol{x}, \boldsymbol{y}), \quad 1 \leq k \leq M
$$


It then invokes the technique of parallel projections to constitute a local approximate solution to Eq. (A.8), which consists of the following recursive sequence

$$
x^{(j)}=\Pi\left(x^{(j-1)}\right)=\sum_{k=1}^{M} \omega_{k} \Pi_{S_{k}}\left(x^{(j-1)}\right),
$$

where $j \geq 1, \boldsymbol{x}^{(0)}$ is the initial estimate, and the weight satisfies $\sum_{k=1}^{M} \omega_{k}=1$. In such a scheme, all the property sets are activated simultaneously and the new iteration is a combination of the projections of the current iterate onto each property set. The following programs implement some of the most useful property mapping operators.

\section{Matrix Rank}

Let $\boldsymbol{X}$ be an arbitrary matrix in $C^{m \times n}$, the metric space consists of all $m \times n$ complex valued matrices, whose singular value decomposition is given by

$$
\boldsymbol{X}=\sum_{j=1}^{r} \sigma_{j} u_{j} v_{j}^{\dagger}=\sigma_{1} u_{1} v_{1}^{\dagger}+\sigma_{2} u_{2} v_{2}^{\dagger}+\cdots+\sigma_{r} u_{r} v_{r}^{\dagger}
$$

where " $\dagger$ " stands for taking the Hermitian conjugate, $r \leq \min (m, n)$ is equal to the rank of $\boldsymbol{X}$, the $\left(\sigma_{j}\right)_{1 \leq j \leq r}$ are real and nonnegative singular values ordered in the monotonically nonincreasing fashion $\sigma_{j} \geq \sigma_{j+1}$, and the $\left(\boldsymbol{u}_{j}\right)_{1 \leq j \leq r}$ and $\left(\boldsymbol{v}_{j}\right)_{1 \leq j \leq r}$ are the corresponding orthonormal left and right singular vectors, respectively. The matrix $\boldsymbol{X}$ is thus constructed from the contribution of $r$ rank-one matrices weighted by the respective singular values. It follows that if $\sigma_{p} \neq \sigma_{p+1}$, the unique matrix $\boldsymbol{X}_{R}$ 
of rank $p$ or less contained in property set $S_{R}$ that lies closest to $\boldsymbol{X}$ in the minimum Frobenius norm sense is given by the norm-reducing closed mapping $\Pi_{S_{R}}$

$$
\boldsymbol{X}_{R}=\Pi_{S_{R}}(\boldsymbol{X})=\sum_{j=1}^{p} \sigma_{j} \boldsymbol{u}_{j} \boldsymbol{v}_{j}^{\dagger}
$$

The nonconvexity of $S_{R}$ is established by noting that the sum of two rank- $p$ matrices can have a rank greater than $p$.

function $Y=$ ste_rank $(Y, M)$

$\%$ function $Y=$ ste_rank $(Y, M)$

$\%$ Set theoretic estimation: matrix rank mapping

$\%====$ arguments $====$

$\% \mathrm{Y}$ : input arbitrary matrix

$\% \mathrm{Y}$ : output matrix with rank=M

$\% \mathrm{M}$ : the desired rank

$\% * * * * * * * * * *$ begin of function ste_rank.m $* * * * * * * * * *$

$[\mathrm{U}, \mathrm{S}, \mathrm{V}]=\operatorname{svd}(\mathrm{Y}, 0)$;

$\mathrm{Y}=\mathrm{U}(:, 1: \mathrm{M}) * \mathrm{~S}(1: \mathrm{M}, 1: \mathrm{M}) * \mathrm{~V}(:, 1: \mathrm{M})^{\prime}$;

return

$\% * * * * * * * * * *$ end of function ste_rank.m $* * * * * * * * * *$

\section{Eigen Structure}

Let $\boldsymbol{X} \in C^{n \times n}$ be a Hermitian matrix whose eigendecomposition is specified by

$$
\boldsymbol{X} \boldsymbol{v}_{j}=\lambda_{j} \boldsymbol{v}_{j}
$$

where $\left(\lambda_{j}\right)_{1 \leq j \leq n}$ are the $n$ real eigenvalues, and $\left(v_{j}\right)_{1 \leq j \leq n}$ are their associated eigenvectors. Let the eigenvalues be ordered in the monotonically nonincreasing fashion 
$\lambda_{j} \geq \lambda_{j+1}$ in which the first $q$ eigenvalues are positive and the rest $(n-q)$ are nonpositive. The unique positive semidefinite matrix $\boldsymbol{X}_{P}$ that lies closest to $\boldsymbol{X}$ in the Frobenius norm sense is given by the following mapping $\Pi_{S_{P}}$ :

$$
\boldsymbol{X}_{P}=\Pi_{S_{P}}(X)=\sum_{j=1}^{q} \lambda_{j} \boldsymbol{v}_{j} \boldsymbol{v}_{j}^{\dagger}
$$

Furthermore, $\Pi_{S_{P}}$ is a norm-reducing closed point-to-point mapping.

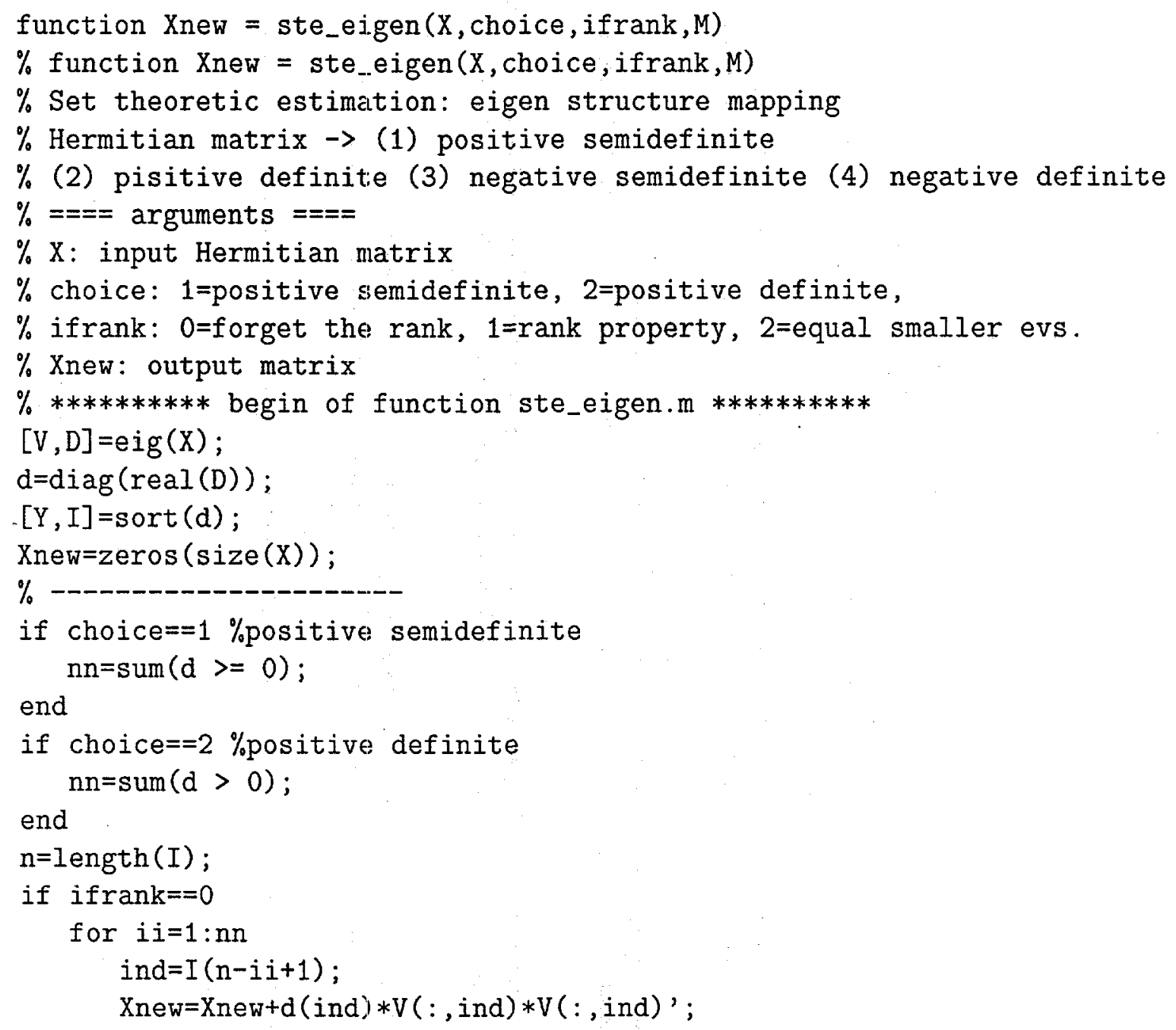




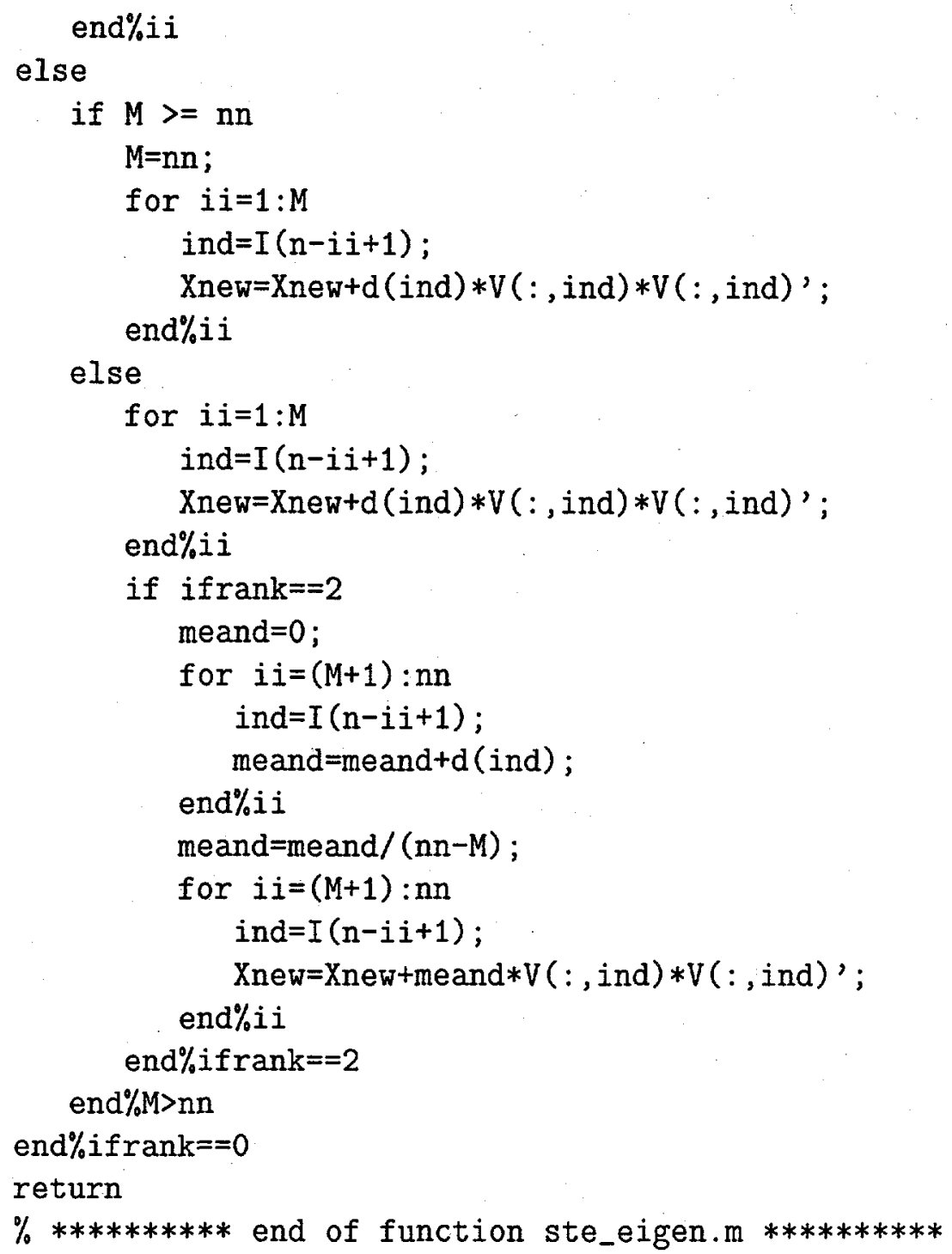

\section{Toeplitz Structure}

A Toeplitz matrix has the property that all the elements along the diagonal are identical and so are those along each subdiagonal. Assume the unique Toeplitz matrix $\boldsymbol{X}_{T}$ that lies closest to an arbitrary matrix $\boldsymbol{X} \in C^{m \times n}$ in the Frobenius norm sense is obtained by the mapping $\Pi_{S_{T}}$. It is found that the Toeplitz-structured mapping 
$\Pi_{S_{T}}$, when applied to $\boldsymbol{X}$, results in $\boldsymbol{X}_{T}$ whose $k$ th subdiagonal elements equal the average value of the $k$ th subdiagonal elements of $\boldsymbol{X}$.

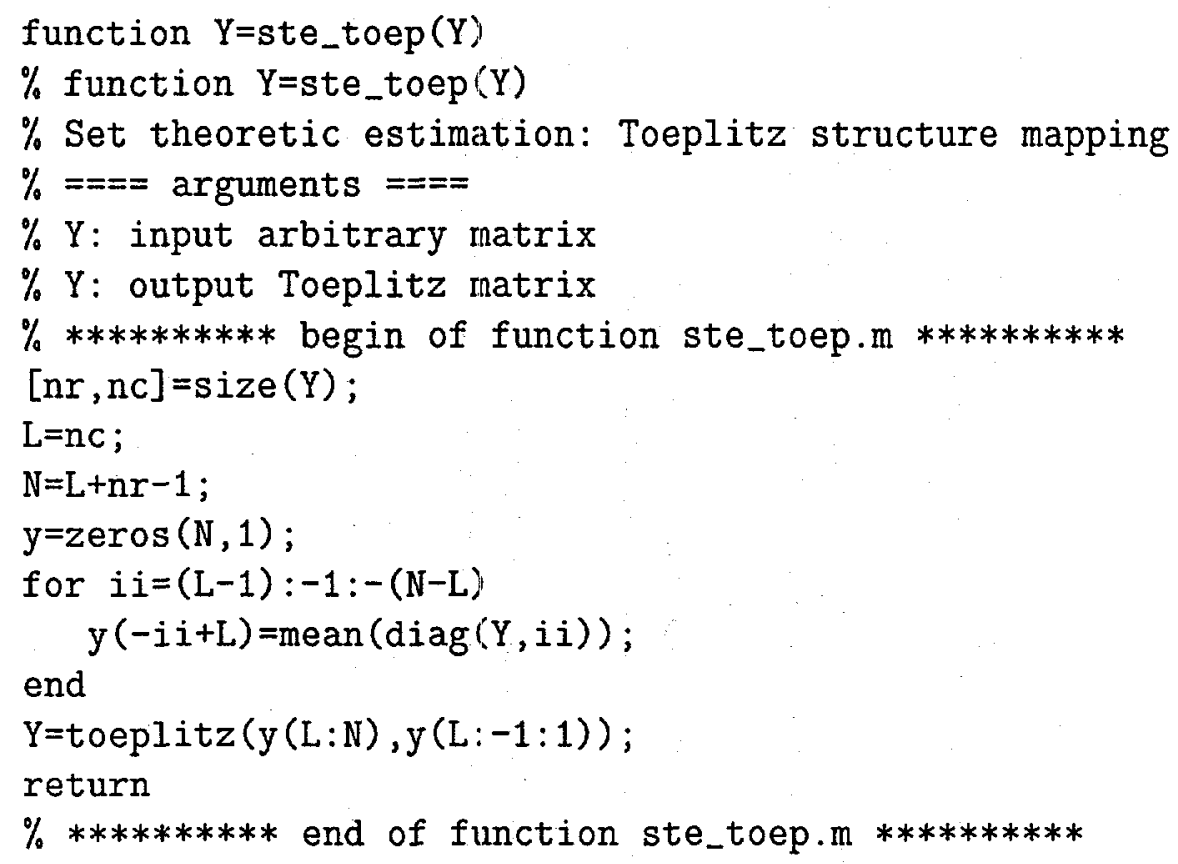

\section{Hermitian Structure}

The unique Hermitian matrix $\boldsymbol{X}_{H}$ that lies closest to a matrix $\boldsymbol{X} \in C^{n \times n}$ in the Frobenius norm sense is given by the following Hermitian-structured mapping $\Pi_{S_{H}}$,

$$
\boldsymbol{X}_{H}=\Pi_{S_{H}}(X)=\frac{1}{2}\left(X+X^{\dagger}\right)
$$

All linear-structured matrix property sets $\left(e . g ., S_{T}\right.$ and $\left.S_{H}\right)$ are both closed and convex. 
function $X=$ ste_herm $(X)$

$\%$ function $X=$ ste_herm $(X)$

$\%$ set theoretic estimation: Hermtian mapping

$\%====$ arguments $====$

$\% \mathrm{X}$ : input arbitrary matrix

$\% \mathrm{X}$ : output Hermitian matrix

$\% * * * * * * * * * *$ begin of function ste_herm.m $* * * * * * * * * *$

$\mathrm{X}=\left(\mathrm{X}+\mathrm{X}^{2}\right) / 2$;

return

$\% * * * * * * * * * *$ end of function ste_herm.m $* * * * * * * * * *$

\section{Subsequence Property}

Assume $\boldsymbol{x}=\{x(1), x(2), \cdots, x(N)\}$ comprises a sum of $Q$ noiseless complex exponentials. This sequence may be decimated by a positive integer $p \geq 2$ to form $p$ subsequences

$$
\boldsymbol{x}_{p: q}=\{x(q), x(p+q), \cdots, x(\operatorname{FIX}(N / p-1) p+q)\} .
$$

Here $1 \leq q \leq p$ and "FIX" denotes rounding toward zero. Each such subsequence will also comprise a sum of $Q$ complex exponentials with the exponents being equal to those of the original raised to the $p^{\text {th }}$ power. We can then construct the composite 
subsequence data matrix $\boldsymbol{X}_{p}$

$$
\boldsymbol{X}_{p}=\left[\begin{array}{c}
\boldsymbol{X}_{p: 1} \\
\boldsymbol{X}_{p: 2} \\
\vdots \\
\boldsymbol{X}_{p: p}
\end{array}\right]
$$

where submatrix $\boldsymbol{X}_{p: q}(1 \leq q \leq p)$ denotes the data matrix formed from subsequence $x_{p: q}$. It follows that $\boldsymbol{X}_{p}$ will also possess rank $Q$. The projector $\Pi_{S_{U, p}}$ associated with the subsequence property set $S_{U, p}$ for an arbitrary standard data matrix $\mathrm{X} \in C^{m \times n}$ can be described as follows. (i) Extract the sequence $\boldsymbol{x}$ from $\boldsymbol{X}$. Decimate $\boldsymbol{x}$ by $p$ to form $p$ subsequences $\left(\boldsymbol{x}_{p: q}\right)_{1 \leq q \leq p}$. (ii) For each $\boldsymbol{x}_{p: q}$, form submatrix $\boldsymbol{X}_{p: q}$, and then construct the composite subsequence data matrix $\boldsymbol{X}_{p}$ by Eq. (A.19). (iii) Find the nearest rank $Q$ approximation to $\boldsymbol{X}_{p}$ by program ste_rank.m. (iv) Perform the inverse operation of (i) and (ii), i.e., extract the submatrix and subsequence and then form the enhanced standard data matrix $\boldsymbol{X}_{U, p}$. Consequently, $\boldsymbol{X}_{U, p}=\Pi_{S_{U, p}}(\boldsymbol{X})$.

function $X=$ ste_sub $\left._{-} X, M, K\right)$

$\%$ function $X=$ ste_sub $(X, M, K)$

$\%$ Set theoretic estimation: subsequence property mapping

$\%====$ arguments $====$

$\% \mathrm{X}$ : input arbitrary Toeplitz data matrix

$\% \mathrm{X}$ : output subsequence-enhanced Toeplitz data matrix

$\% \mathrm{M}: \#$ of exponential components (integer, $1 * 1$ )

$\% \mathrm{~K}$ : decimation number (integer, $1 * 1$ )

$\% * * * * * * * * * *$ begin of function ste_sub.m $* * * * * * * * *$

$\%$--- extract the original time series 
$[\mathrm{nr}, \mathrm{nc}]=\operatorname{size}(\mathrm{X})$;

$\mathrm{L}=\mathrm{nc}-1 ; \%$ the data matrix will be $(\mathrm{N}-\mathrm{L}) *(\mathrm{~L}+1)$

$\mathrm{N}=\mathrm{nr}+\mathrm{L} ; \%$ length of original time series

$y=\operatorname{zeros}(1, N)$;

for $i i=1: N$

$$
y(i i)=\operatorname{mean}(\operatorname{diag}(X,-i i+L+1)) \text {; }
$$

end

$\mathrm{Nt}=\mathrm{fix}($ length $(\mathrm{y}) / \mathrm{K}) * \mathrm{~K} ; \%$ length of the truncated time series

$\mathrm{nn}=\mathrm{Nt} / \mathrm{K} ; \%$ length of the decimated time series

$11=f l o o r(n n / 2) ; \%$ sub data matrix will be $(n n-11) *(11+1)$

$\mathrm{Xk}=\operatorname{zeros}((\mathrm{nn}-11) * \mathrm{~K}, 11+1)$;

$\%$--- construct composite data matrix

ynew=zeros $(1, \mathrm{Nt})$;

for $\mathrm{mm}=1: \mathrm{K}$

$\mathrm{ykm}=\mathrm{y}(\mathrm{mm}: \mathrm{K}: \mathrm{Nt})$;

$\mathrm{Xk}(((\mathrm{nn}-11) *(\mathrm{~mm}-1)+1):((\mathrm{nn}-11) * \mathrm{~mm}),:)=\ldots$ toeplitz $(y k m(1 l+1: n n), \operatorname{rot} 90(y k m(1: 1 l+1), 2))$;

end

$\%$--- rank projection

$\mathrm{Xk}=\mathrm{mserank}(\mathrm{Xk}, \mathrm{M})$;

$\%$--- extract enhanbced subsequence

for $\mathrm{mm}=1: \mathrm{K}$

$y k m=\operatorname{zeros}(1, \mathrm{nn})$;

for $i i=1: n n$ $y \operatorname{km}(i i)=\ldots$

$\operatorname{mean}(\operatorname{diag}(X k(((n n-1)) *(m m-1)+1):((n n-11) * m m),:),-i i+11+1))$;

end

ynew $(\mathrm{mm}: \mathrm{K}: \mathrm{Nt})=\mathrm{ykm}$;

end

if $\mathrm{N}==\mathrm{Nt}$

else

ynew $=[$ ynew y $(\mathrm{Nt}+1: \mathrm{N})]$;

end

$\%$--- corstruct enhanced data matrix

$X=$ toeplitz $($ ynew $(L+1: N), \operatorname{rot} 90($ ynew $(1: L+1), 2))$;

return

$\% * * * * * * * *$ end of function ste_sub.m 


\section{A.3 System Identification}

An important application of system identification in NMR is the prediction and extension of truncated FID. A FID $x(n)$ is known over a given interval of time, and the goal is to determine $x(n)$ over some other interval. If a model can be found that provides an accurate representation for the system that generates $x(n)$, then the model may be used to estimate the unknown values of $x(n)$. In general, there are two steps in the modeling process. The first is to choose an appropriate parametric form for the model. In system identification, one frequently assumes that the model has a rational system function of the form

$$
H(z)=\frac{B_{q}(z)}{A_{p}(z)}=\frac{\sum_{k=0}^{q} b_{q}(k) z^{-k}}{1+\sum_{k=1}^{p} a_{p}(k) z^{-k}}
$$

Once the form of the model has been selected, the next step is to find the model parameters that provide the best approximation to the given FIDs.

\section{A.3.1 Padé Approximation}

Given a FID $x(n)$, the Padé approximation finds the coefficients in the model of Eq. (A.20) by matching the first $p+q+1$ FID data points exactly, i.e., $x(n)=h(n)$ for $n=0,1, \cdots, p+q$, where $h(n)$ is the unit response function. There are three required inputs to this program. The first is the vector $\mathrm{x}$ that contains the values of the FID $x(n)$ that is to be modeled. The other two inputs, $\mathrm{p}$ and $\mathrm{q}$, specify the 
model order, i.e., the order of the polynomials $A_{p}(z)$ and $B_{q}(z)$, respectively. The output consists of two vectors, a and b, that contain the model coefficients $a_{p}(k)$ and $b_{q}(k)$, respectively. As indicated in Eq. (A.20), the first coefficient of the vector a will always be equal to one.

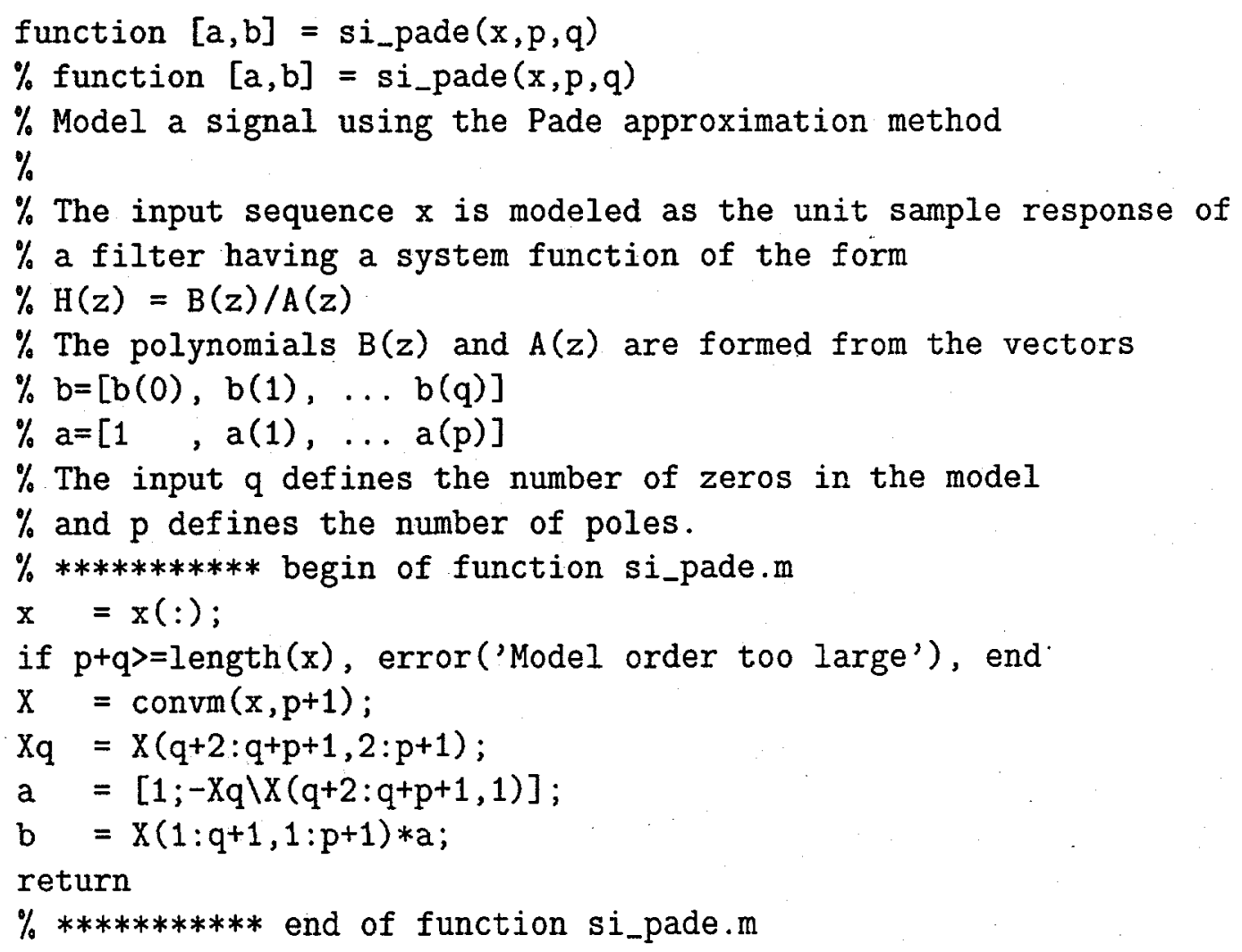

function $X=\operatorname{convm}(x, p)$

$\%$ function $x=\operatorname{convm}(x, p)$

$\%$ Generates a convolution matrix

$\%$

$\%$ Given a vector $\mathrm{x}$ of lenght $\mathrm{N}$, an $\mathrm{N}+\mathrm{p}-1$ by $\mathrm{p}$ convolution

$\%$ matrix of the following form is generated

$\%$

$\%$

$1 \mathrm{x}(0) 0$

0 


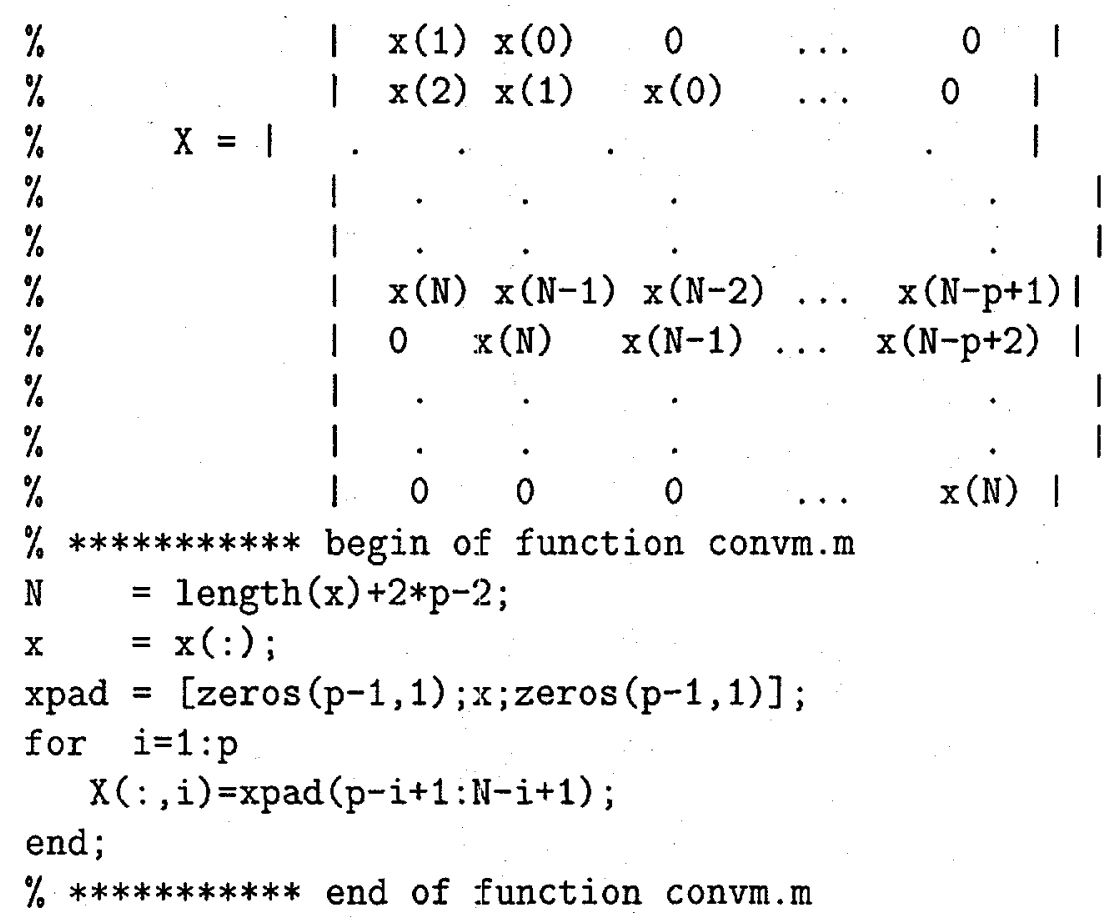

\section{A.3.2 Prony Method}

As with Padé approximation, Prony method finds a model for a FID $x(n)$ of the form given in Eq. (A.20). Unlike the Padé approximation, however, the denominator coefficients $a_{p}(k)$ are found by minimizing the Prony error $\varepsilon_{\text {Prony }}$ defined as

$$
\varepsilon_{\text {Prony }}=\sum_{n=q+1}^{\infty}|e(n)|^{2}=\sum_{n=q+1}^{\infty}\left|x(n)+\sum_{l=1}^{p} a_{p}(l) x(n-l)\right|^{2}
$$

Once the denominator coefficients have been determined, the numerator coefficients $b_{q}(k)$ are found using the Padé approximation to match the FID exactly for the first $q+1$ values of $x(n)$.

In the derivation of Prony method, it is assumed that the FID $x(n)$ is known for all 
$n \geq 0$. Therefore, there is an important practical issue that concerns how to address the problem of only being able to record and process a finite-length observation of $x(n)$. In the following program, we assume that the FID $x(n)$ is zero for all values of $n$ that are greater than the length of the input vector $\mathrm{x}$. Therefore, it uses the autocorrelation method (described below) to find the denominator coefficients, and the Padé approximation to find the numerator coefficients.

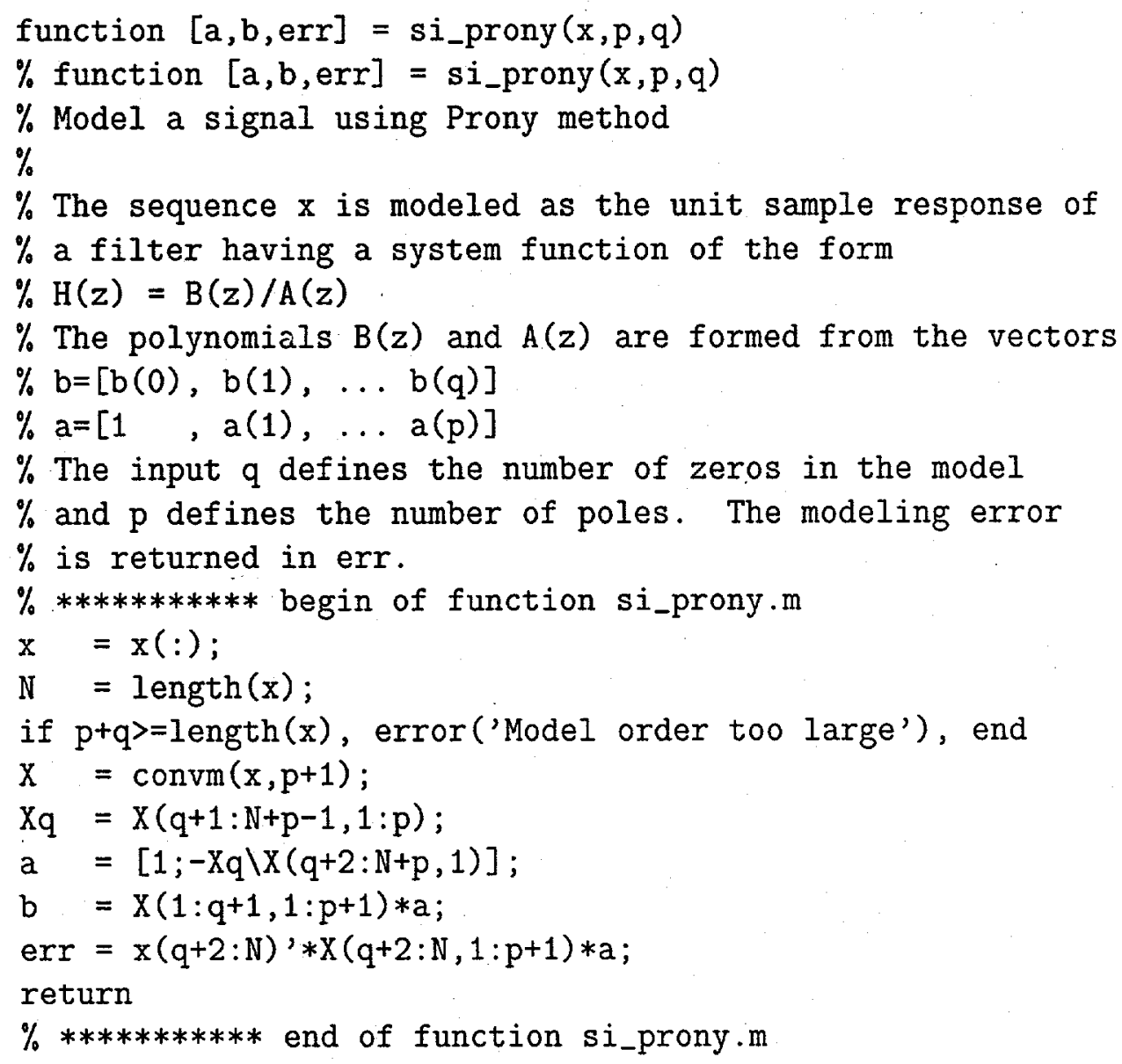




\section{A.3.3 Iterative Prefiltering}

Iterative prefiltering, or the method of Steiglitz and McBride, is an iterative algorithm to find the rational model of Eq. (A.20) for a FID $x(n)$ that minimizes the least squares error

$$
\varepsilon_{L S}=\sum_{n=0}^{\infty}|x(n)-h(n)|^{2}
$$

where $h(n)$ is the unit response function. Although there is no general proof of convergence, iterative prefiltering often reaches an acceptable solution after 5 to 10 iterations. The inputs that are required are the signal vector, $\mathrm{x}$, the number of poles in the model, $\mathrm{p}$, the number of zeros, $\mathrm{q}$, and the number of iterations $\mathrm{n}$. In addition, there is an optional input vector, a0, that is used to initialize the recursion with a given set of denominator coefficients. If this input is left unspecified, then the initial condition is found using Prony method. The outputs of the program are the model coefficients $a_{p}(k)$ and $b_{q}(k)$, which are stored in the vectors a and b, respectively, and the squared error, err.

function $[a, b, e r r]=$ si_iter $(x, p, q, n, a)$

$\%$ function $[a, b, e r r]=\operatorname{si} i \operatorname{ter}(x, p, q, n, a)$

$\%$ Pole-zero signal modeling using iterative prefiltering. $\%$

$\%$ The sequence $x$ is modeled as the unit sample response of

$\%$ a filter having a system function of the form

$\% H(z)=B(z) / A(z)$

$\%$ The polynomials $B(z)$ and $A(z)$ are formed from the vectors 


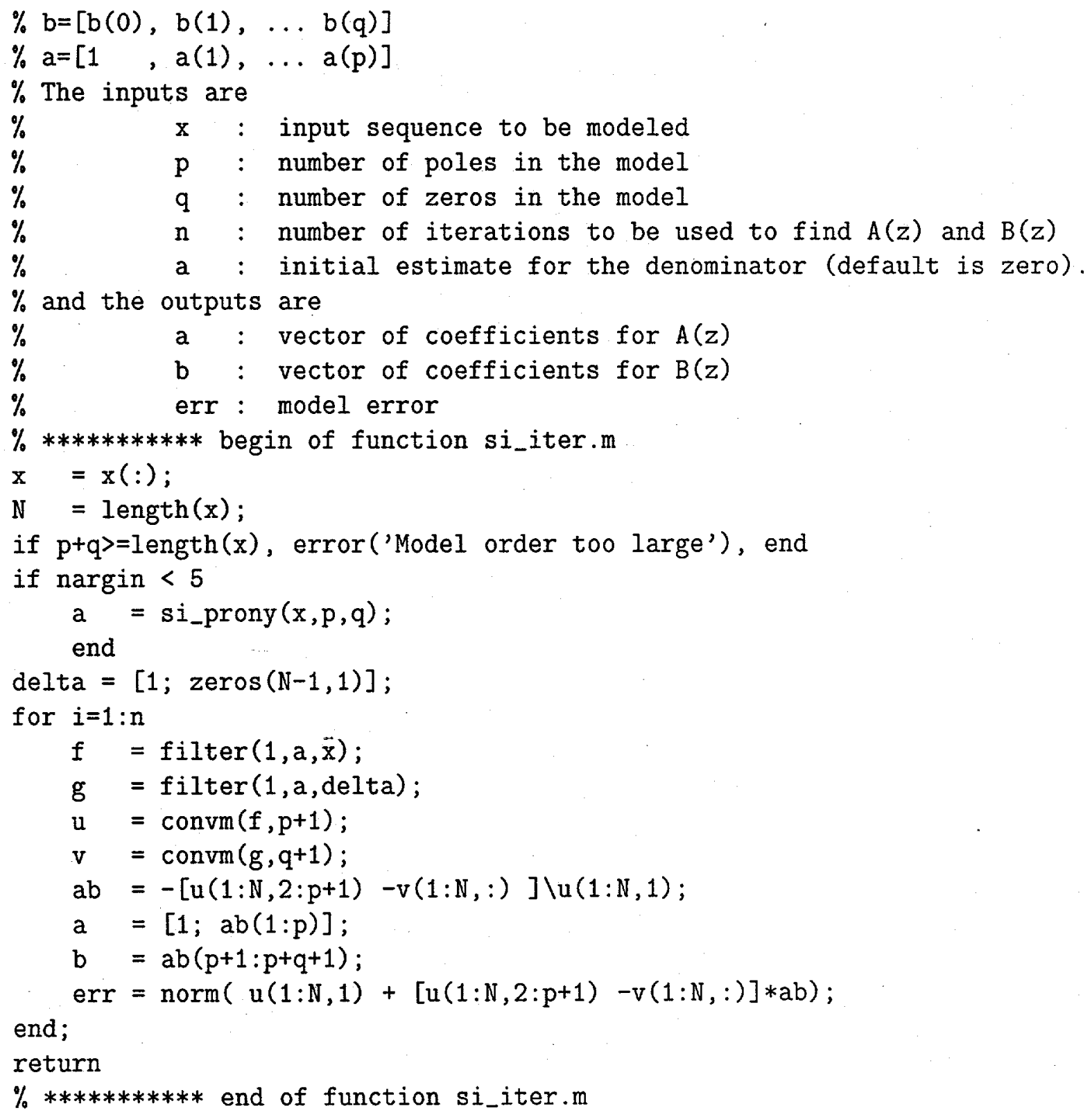

\section{A.3.4 Autocorrelation Method}

The autocorrelation method is an all-pole modeling technique that finds the allpole coefficients $a_{p}(k)$ from the values of $x(n)$ for $n=0,1, \cdots, N$ by minimizing the 
Prony error Eq. (A.21). Since $x(n)$ is assumed to be known only for $0 \leq n \leq N$, the error $e(n)$ may only be evaluated for $p \leq n \leq N$. Therefore, $\varepsilon_{\text {Prony }}$ can not be minimized unless some assumptions are made about the values of $x(n)$ outside the interval $[0, N]$. With the autocorrelation method, $x(n)$ is assumed to be equal to zero for $n<0$ and $n>N$, which is equivalent to applying a rectangular data window to $x(n)$. Although this window biases the solution, it ensures that the all-pole model will be stable.

The input $\mathrm{x}$ is a vector that contains the signal values $x(n)$, and $\mathrm{p}$ is an integer that specifies the model order (number of poles). The output a is the vector of coefficients $a_{p}(k)$, and err is the modeling error, $\epsilon_{\text {Prony }}=\min \left\{\varepsilon_{\text {Prony }}\right\}$.

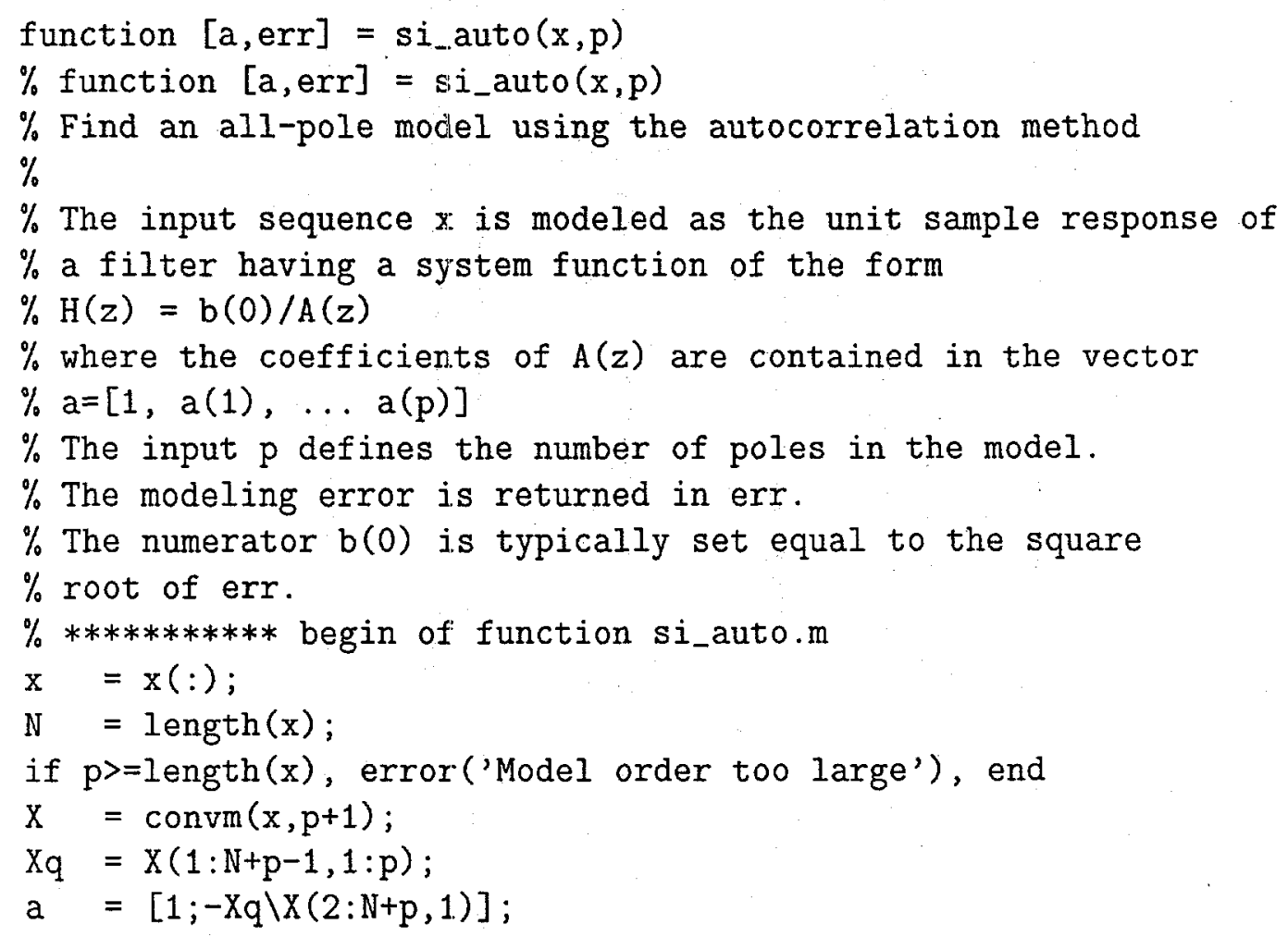


$\operatorname{err}=\operatorname{abs}\left(X(1: N+p, 1)^{\prime} * X * a\right)$;

return

$\% * * * * * * * * * * *$ end of function si_auto.m

\section{A.3.5 Covariance Method}

The covariance method is an alternative to the autocorrelation method for finding an all-pole model for a finite-length sequence $x(n), n=0,1, \cdots, N$. Instead of assuming that the unknown values of $x(n)$ are equal to zero, the covariance method modifies the error that is to be minimized. The modification simply involves redefining the limits on the sum for $\varepsilon_{\text {Prony }}$ to begin at $n=p$ and end at $n=N$. Since the covariance method does not window the data, the model is generally more accurate than the autocorrelation method. However, the model is not guaranteed to be stable.

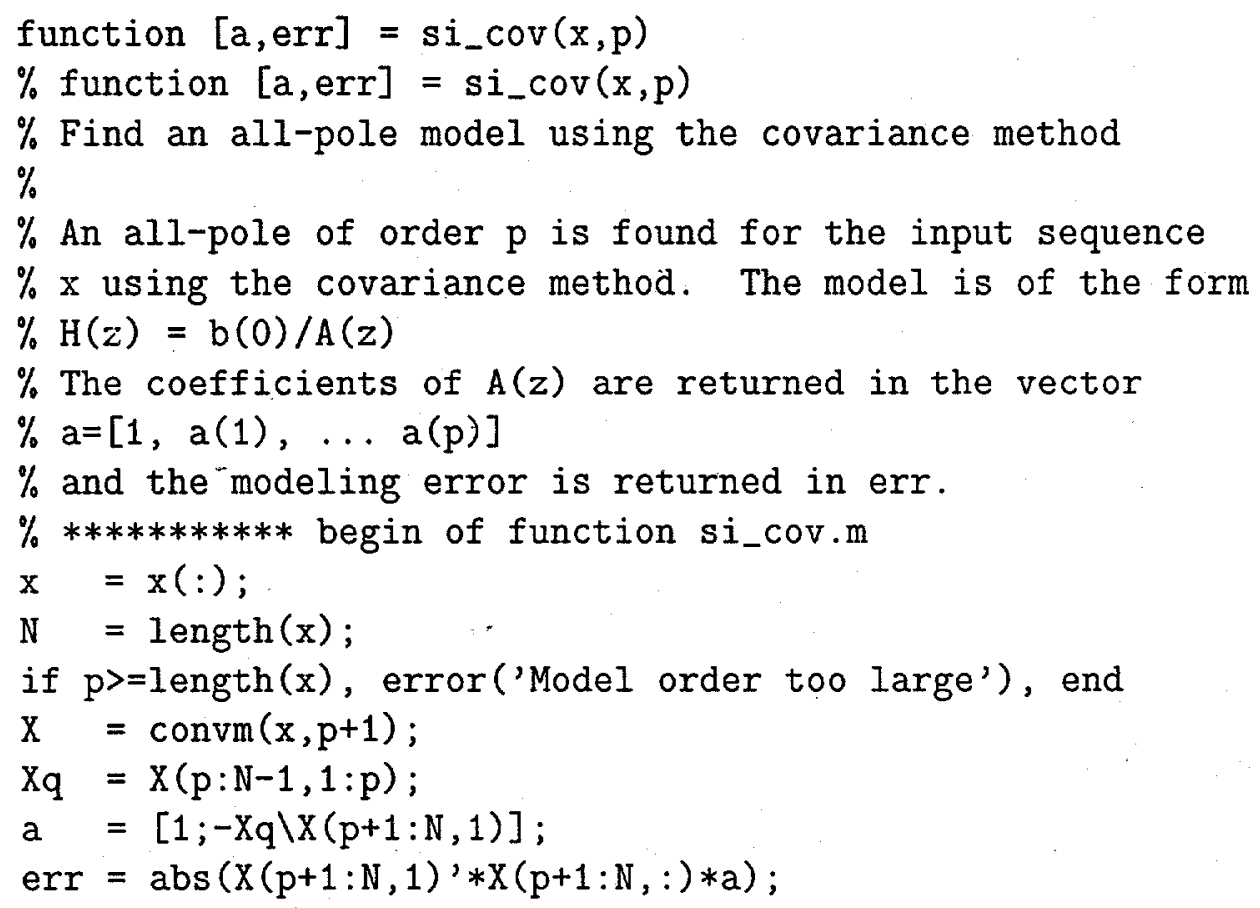


return

$\% * * * * * * * * * * *$ begin of function si_cov.m

\section{A.4 Spectral Estimation}

In this section, we consider the problem of estimating the frequency spectra of NMR FIDs. If the FID $x(n)$ is known for all $n$, estimating the frequency spectrum is straightforward, in theory, since it can be computed by its Fourier transform. However, there are two practical difficulties that make spectral estimation both an interesting and a challenging task. First, the amount of FID points may be very limited. Secondly, the FID is often corrupted by noise or contaminated with an interfering signal. Thus, NMR spectral estimation is a problem that involves estimating the frequency spectrum $P_{x}\left(e^{j \omega}\right)$ from a finite number of noisy measurements of the FID $x(n)$.

The approaches for spectral estimation may be generally categorized into two classes: classical (nonparametric) and nonclassical (parametric). In the first class, the frequency spectrum is estimated by directly Fourier transforming the FID, while the second class is based on using a model for process which drives the FID. For example, if it is known that $x(n)$ is a $p$ th-order autoregressive process, then measured values of $x(n)$ may be used to estimate the parameters of the all-pole model, $a_{p}(k)$, and these estimated model parameters, $\hat{a}_{p}(k)$, may then, in turn, be used to estimate 
$P_{x}\left(e^{j \omega}\right)$ as follows:

$$
P_{x}\left(e^{j \omega}\right)=\frac{1}{\left|\sum_{k=0}^{p} \hat{a}_{p}(k) e^{-j k \omega}\right|^{2}},
$$

where $j=\sqrt{-1}$.

\section{A.4.1 Maximum Likelihood Method}

For a FID $x(n)$, the maximum likelihood estimate of the frequency spectrum is

$$
P_{M L M}\left(e^{j \omega}\right)=\frac{1}{\boldsymbol{e}^{H} \boldsymbol{R}_{x}^{-1} \boldsymbol{e}}
$$

where $\boldsymbol{R}_{x}$ is the $p \times p$ autocorrelation matrix and $\boldsymbol{e}=\left[1, e^{j \omega}, \cdots, e^{j p \omega}\right]$.

Instead of evaluating Eq. (A.24) directly, the following program finds the maximum likelihood estimate using the expansion

$$
P_{M L M}\left(e^{j \omega}\right)=\frac{1}{\sum_{i=1}^{p} \frac{1}{\lambda_{i}}\left|e^{H} v_{i}\right|^{2}}
$$

where $\lambda_{i}$ and $\boldsymbol{v}_{i}$ are the eigenvalues and eigenvectors, respectively, of $\boldsymbol{R}_{x}$.

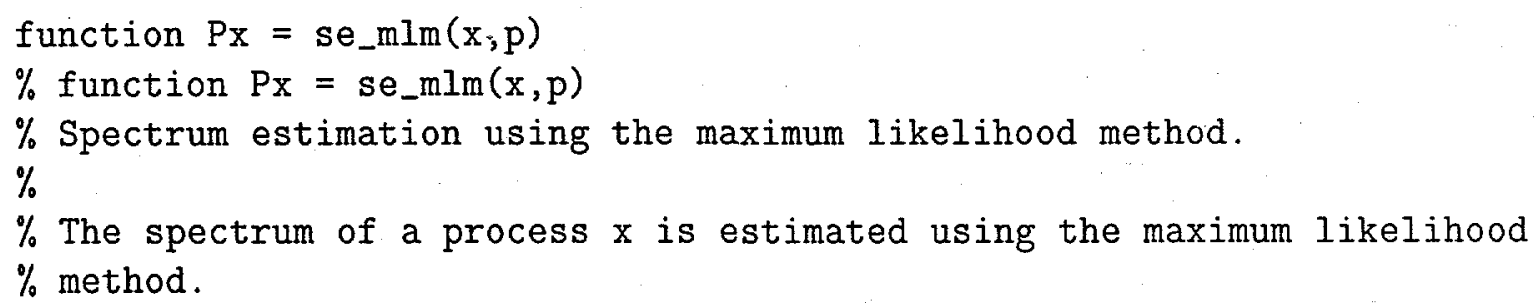


$\% \mathrm{x}:$ Input sequence

$\% \mathrm{p}:$ : Order of the minimum variance estimate - for short

$\%$ sequences, $p$ is typically about length $(x) / 3$

$\%$ The spectrum estimate is returned in $\mathrm{Px}$ using a $\mathrm{dB}$ scale.

$\% * * * * * * * * * * *$ begin of function se_mlm.m

$\mathrm{x}=\mathrm{x}(:)$;

$\mathrm{R}=\operatorname{covaiance}(\mathrm{x}, \mathrm{p})$;

$[\mathrm{v}, \mathrm{d}]=\operatorname{eig}(\mathrm{R})$;

$\mathrm{U}=\operatorname{diag}(\operatorname{inv}(\operatorname{abs}(\mathrm{d})+\mathrm{eps}))$;

$\mathrm{V}=\operatorname{abs}(\mathrm{fft}(\mathrm{v}, 1024)) \cdot{ }^{-2}$;

$\mathrm{Px}=10 * \log 10(\mathrm{p})-10 * \log 10(\mathrm{~V} * \mathrm{U})$;

return

$\% * * * * * * * * * * *$ end of function si_pade.m

\section{A.4.2 Maximum Entropy Method}

The maximum entropy method of spectral estimation finds an all-pole model for a process using the autocorrelation method, and then uses the model parameters $a_{p}(k)$ to estimate the spectrum as follows:

$$
P_{M E M}\left(e^{j \omega}\right)=\frac{\epsilon_{\text {Prony }}}{\left|1+\sum_{k=1}^{p} a_{p}(k) e^{-j k \omega}\right|^{2}}
$$

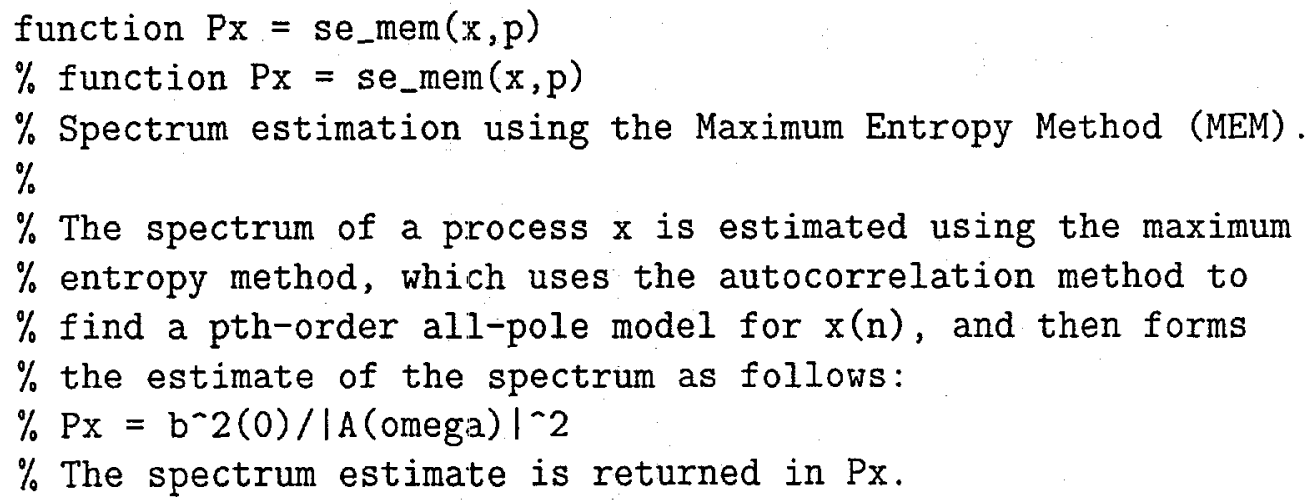




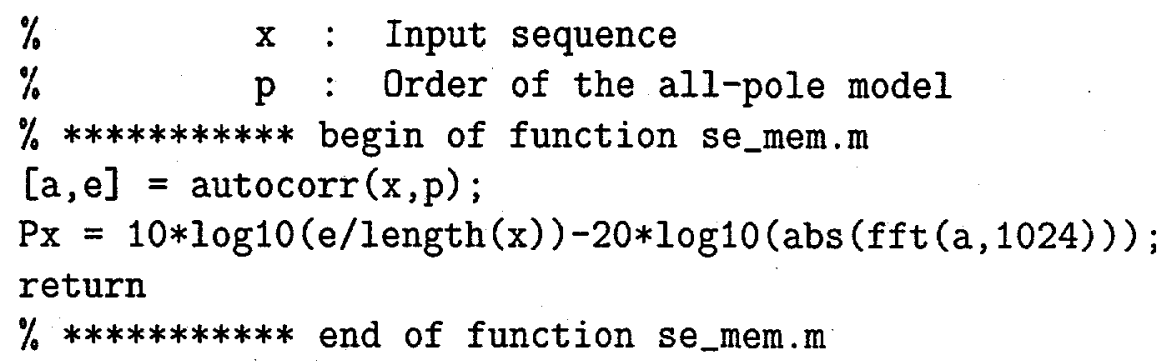

\section{A.4.3 Noise Subspace Methods}

In this subsection, we describe the programs that are used for FIDs that consist of a sum of complex exponentials in white noise. We begin with the noise subspace methods, which are frequency estimation algorithms and include the Pisarenko harmonic decomposition, the MUSIC algorithm, the eigenvector method, and the minimum norm algorithm. In the next subsection, we discuss the program that use a signal subapace approach, which involves a principal component analysis of the autocorrelation matrix $\boldsymbol{R}_{x}$.

\section{Pisarenko Harmonic Decomposition}

A noise subspace method to estimate the frequencies of $p$ complex exponentials in noise involves the use of a frequency estimation of the form

$$
P_{x}\left(e^{j \omega}\right)=\frac{1}{\sum_{i=p+1}^{M} \alpha_{i}\left|e^{H} v_{i}\right|^{2}}
$$


where $\boldsymbol{v}_{\boldsymbol{i}}$ are vectors that lie in the noise subspace of $\boldsymbol{R}_{x}$, and $\alpha_{i}$ are constants. In the Pisarenko harmonic decomposition, the frequency estimation function is

$$
P_{\text {Pisarenko }}\left(e^{j \omega}\right)=\frac{1}{\left|e^{H} v_{m i n}\right|^{2}}
$$

where $\boldsymbol{v}_{\min }$ is the eigenvector of $\boldsymbol{R}_{x}$ that has the minimum eigenvalue. The input of the program is the FID vector, $x$, and an interger $p$ that defines the number of complex exponentials in $x(n)$. The output is the eigenvector having the smallest eigenvalue, a, along with the minimum eigenvalue, sigma, which may be used as an estimate of the white noise variance.

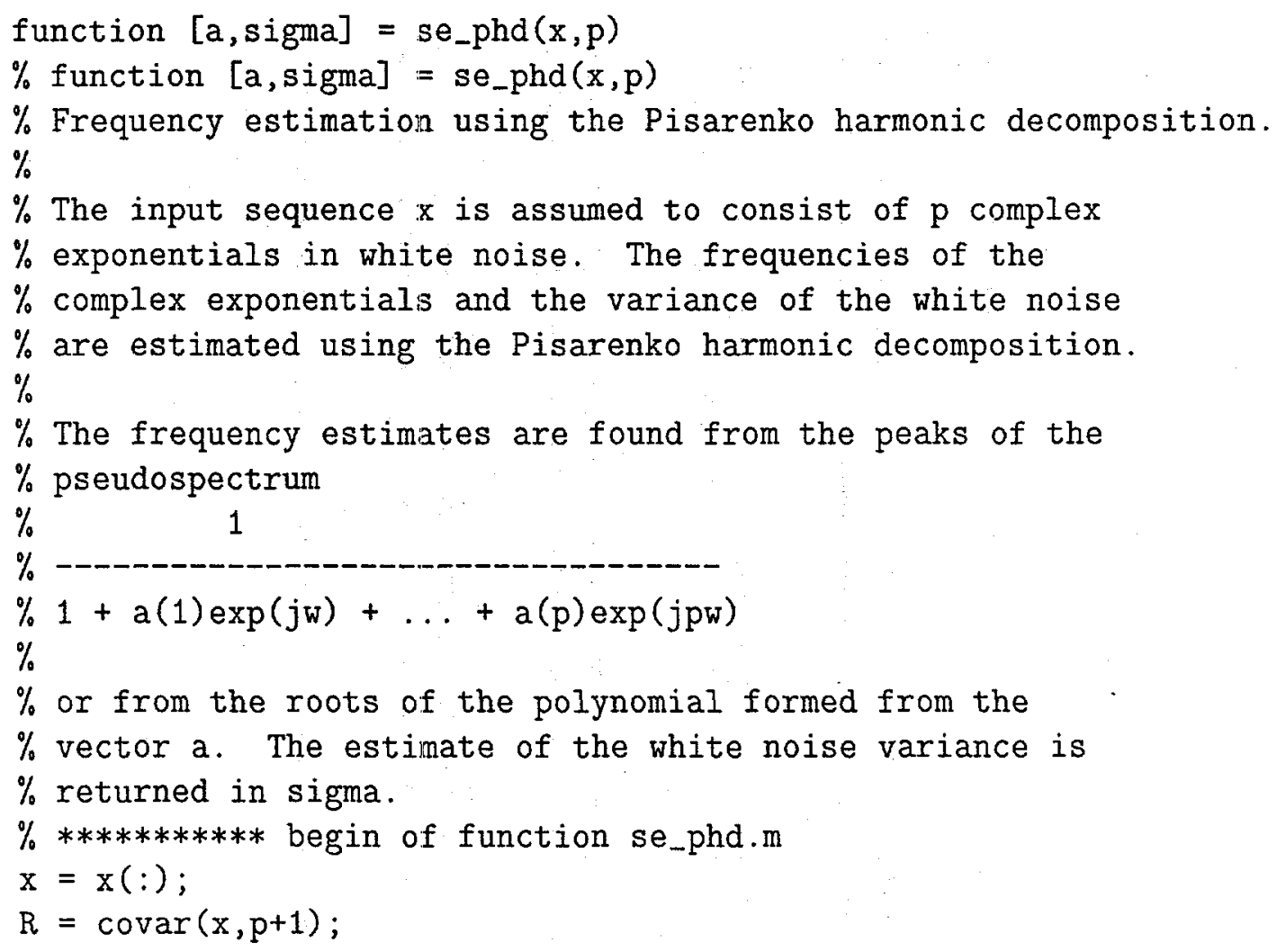


$[v, d]=e i g(R)$;

$\operatorname{sigma}=\min (\operatorname{diag}(d))$;

index $=f$ ind $(\operatorname{diag}(d)==$ sigma) ;

$\mathrm{a}=\mathrm{v}(:$, index $)$;

return

$\% * * * * * * * * * * *$ end of function se_phd.m

\section{MUSIC Method}

The MUSIC algorithm is another frequency estimation technique of the form given in Eq. (A.27). The constants $\alpha_{i}$ are equal to one and $\boldsymbol{v}_{i}$ are the $M-p$ eigenvectors of $\boldsymbol{R}_{x}$ that have the smallest eigenvalues.

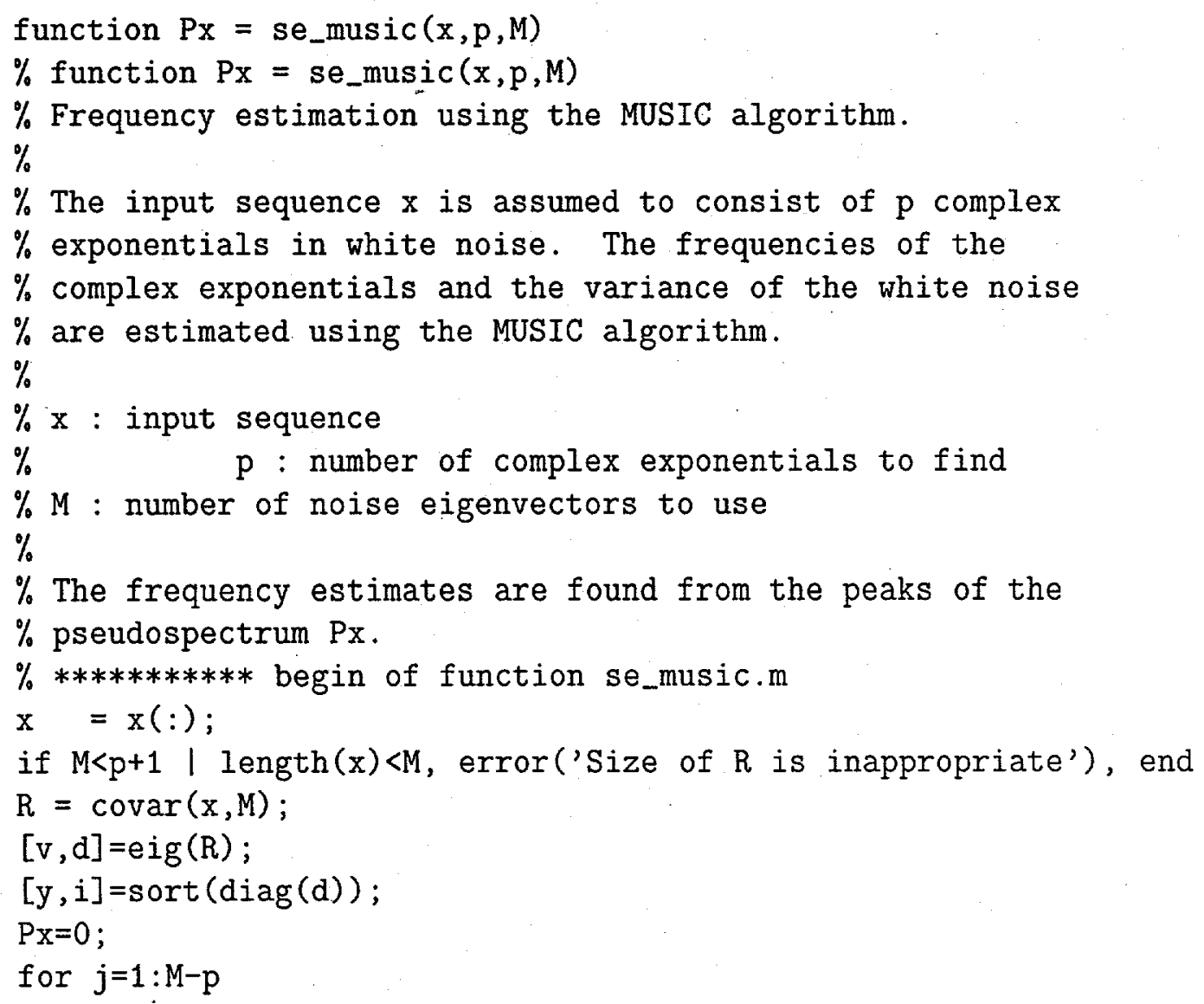


$P x=P x+a b s(f f t(v(:, i(j)), 1024))$

end;

$P x=-20 * \log 10(P x)$;

return

$\% * * * * * * * * * * *$ end of function se_music.m

\section{Eigenvector Method}

The eigenvector method is a frequency estimation algorithm that is similar to the MUSIC algorithm. As wuth the MUSIC algorithm, the vectors $v_{i}$ for $i=p+1$ to $M$ are the eigenvectors that have the smallest eigenvalues. The constants, $\alpha_{i}$, however, are equal to the inverse of the eigenvalues, $\alpha_{i}=1 / \lambda_{i}$.

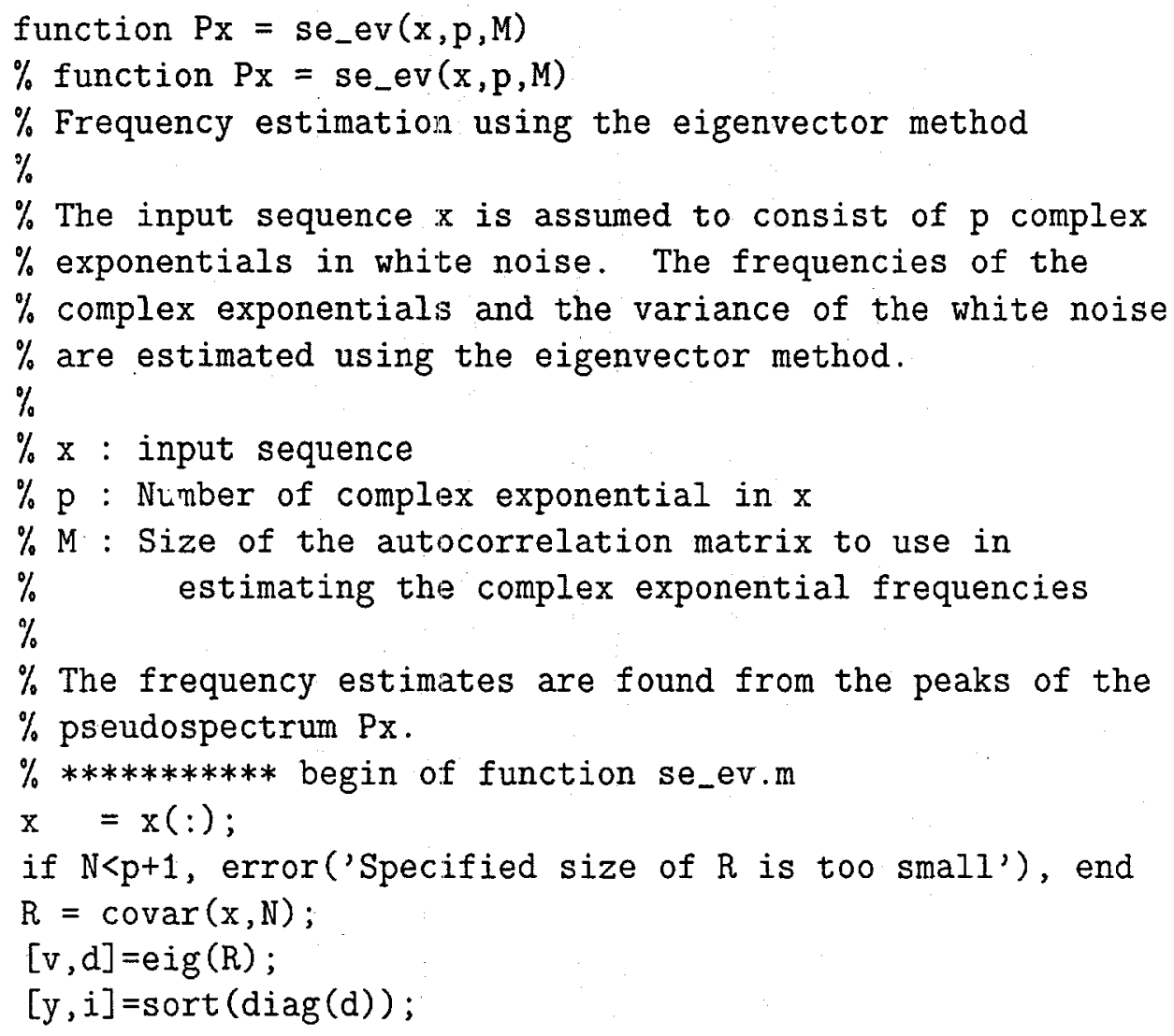




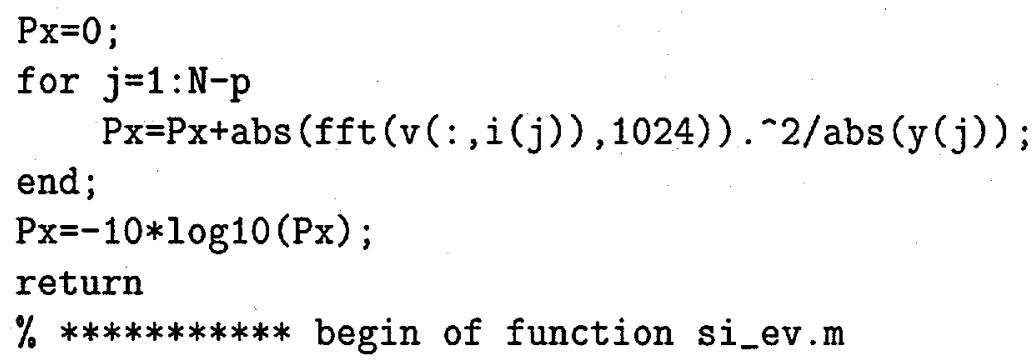

\section{Minimum Norm Method}

The last frequency estimation algorithm included in this package is the minimum norm method, which uses a frequency estimation function of the same form as that used for the Pisarenko harmonic decomposition,

$$
P_{M N}\left(e^{j \omega}\right)=\frac{1}{\left|e^{H} a\right|^{2}}
$$

However, instead of using the eigenvector having the smallest eigenvalue, the minimum norm method uses the vector $\boldsymbol{a}$ in the noise subspace that has the minimum norm.

function $P x=$ se_minnorm $(x, p, M)$

$\%$ function $P x=$ se_minnorm $(x, p, M)$

$\%$ Frequency estimation using the minimum norm algorithm.

$\%$

$\%$ The input sequence $\mathrm{x}$ is assumed to consist of $\mathrm{p}$ complex

$\%$ exponentials in white noise. The frequencies of the

$\%$ complex exponentials and the variance of the white noise

$\%$ are estimated using the minimum norm algorithm.

$\%$

$\% \mathrm{x}$ : input sequence

$\% \mathrm{p}:$ Number of complex exponential in $\mathrm{x}$ 
$\%$ : Size of the autocorrelation matrix to use in

$\%$ estimating the complex exponential frequencies

$\%$

$\%$ The frequency estimates are found from the peaks of the

$\%$ pseudospectrum $\mathrm{Px}$.

$\%$

$\% * * * * * * * * * * *$ begin of function se_minnorm.m

$\mathrm{x}=\mathrm{x}(:)$;

if $N<p+1$, error('Specified size of $R$ is too small'), end $\mathrm{R}=\operatorname{covar}(\mathrm{x}, \mathrm{N})$;

$[\mathrm{v}, \mathrm{d}]=\mathrm{e} i \mathrm{~g}(\mathrm{R})$;

$[y, i]=\operatorname{sort}(\operatorname{diag}(d))$;

for $j=1: N-p$

$V=[V, v(:, i(j))]$;

end;

$\mathrm{a}=\mathrm{V} * \mathrm{~V}(1,:)^{\prime} ;$

$P x=-20 * \log 10(\operatorname{abs}(f f t(a, 1024)))$;

return

$\% * * * * * * * * * * *$ begin of function si_minnorm.m

\section{A.4.4 Signal Subspace Method}

For $\mathrm{s}$ NMR FID consisting of $p$ complex exponentials in noise, a signal subspace method of spectral estimation finds a reduced rank approximation to the autocorrelation matrix using the $p$ principal eigenvectors and eigenvalues,

$$
\hat{\boldsymbol{R}}_{x}=\sum_{i=1}^{p} \lambda_{i} \boldsymbol{v}_{i} \boldsymbol{v}_{i}^{H}
$$

and then estimates the frequency spectrum from $\hat{\boldsymbol{R}}_{x}$, as described previously.

function $\mathrm{Px}=$ se_principal $(\mathrm{x}, \mathrm{p}, \mathrm{M})$

$\%$ function $\mathrm{Px}=$ se_principal $(\mathrm{x}, \mathrm{p}, \mathrm{M})$

$\%$ 
$\%$ The spectrum of a process $\mathrm{x}$ is estimated using a principal

$\%$ components analysis of the autocorrelation matrix.

$\%$ The model for the process is that $x(n)$ consists of a sum of

$\%$ complex exponentials in white noise.

$\%$ After a principle components analysis, the principal eigenvectors

$\%$ are used in the Blackman-Tukey estimate.

$\%$

$\% \quad x \quad$ : input sequence

$\% \quad p \quad$ : number of complex exponentials in $x$

$\% \quad M \quad$ : size of autocorrelation matrix

$\%$ The spectrum estimate is returned in Px using a $d B$ scale.

$\% * * * * * * * * * * *$ begin of function se_principal.m

$\mathrm{x}=\mathrm{x}(:)$;

if $M<p+1$, error('Specified size of $R$ is too small'), end

$\mathrm{R}=\operatorname{covar}(\mathrm{x}, \mathrm{M})$;

$[\mathrm{v}, \mathrm{d}]=e \mathrm{ig}(\mathrm{R})$;

$[y, i]=\operatorname{sort}(\operatorname{diag}(d))$;

$\mathrm{Px}=0$;

for $j=M-p+1, M$;

$P x=P x+a b s(f f t(v(:, i(j)), 1024)) * \operatorname{sqrt}(\operatorname{real}(y(j)))$;

end;

$P x=20 * \log 10(P x)-10 * \log 10(M)$;

return

$\% * * * * * * * * * * *$ end of function se_principal.m 


\section{Bibliography}

[1] S. M. Kay, "Modern Spectral Estimation," Prentice-Hall, Englewood Cliffs, New Jersey, 1987.

[2] S. L. Marple, "Digital Spectral Analysis with Applications," Prentice-Hall, Englewood Cliffs, New Jersey, 1987.

[3] J. C. Lindon and A. G. Ferrige, Prog. NMR Spectrosc. 14, 27 (1980).

[4] R. de Beer and D. van Ormondt, in "NMR Basic Principles and Progress" (P. Diehl, E. Fluck, H. Gunther, R. Kosfeld, and J. Seelig, Eds.), Vol. 26, pp. 201, Springer-Verlag, Berlin/Heidelberg, 1992.

[5] R. E. Hoffman and G. C. Levy, Prog. NMR Spectrosc. 23, 211 (1991).

[6] J. J. Led and H. Gesmar, Chem. Rev. 91, 1413 (1991).

[7] R. Roy, B. G. Sumpter, G. A. Pfeffer, S. K. Gray, and D. W. Noid, Physics Reports 205, 109 (1991).

[8] H. Gesmar, J. J. Led, and F. Abildgaard, Prog. NMR Spectrosc. 22, 255 (1990). 
[9] D. S. Stephenson, Prog. NMR Spectrosc. 20, 515 (1988).

[10] H. Barkhuijsen, R. de Beer, A. C. Drogendijk, D. van Ormondt, and J. W. C. van der Veen, in "Proceedings International School of Physics 'Enrico Fermi' on the 'Physics of NMR Spectroscopy in Biology and Medicine'" (B. Maraviglia, Ed.), pp. 313, Italian Physical Society, 1988.

[11] The Mathworks, Inc., "MATLAB Reference Guide," South Natick, Massachusetts, 1992.

[12] J. J. Dongarra, C. B. Moler, J. R. Bunch, and G. W. Steward, "LINPACK User's Guide," SIAM, Philadelphia, 1979.

[13] B. T. Smith, J. M. Boyle, J. J. Dongarra, B. S. Garbow, Y. Ikebe, V. C. Klema, and C. B. Moler, "Matrix Eigensystem Routines-EISPACK Guide," SpringerVerlag, Berlin, 1976.

[14] B. P. O. van Tongeren, A. F. Mehlkopf, D. van Ormondt, M. Schramp, and R. de Beer, J. Magn. Reson. 100, 619 (1992).

[15] H. Park, J. B. Rosen, and S. van Huffel, in "SVD and Signal Processing III: Algorithms, Architectures, and Applications" (M. Moonen and B. De Moor, Eds.), pp. 399-406, Elsevier Science, New York, 1995.

[16] T. J. Abatzoglou, J. M. Mendel, and G. A. Harada, IEEE Trans. Signal Process. 39, 1070 (1991). 
[17] J. H. McClellan and D. Lee, IEEE Trans. Signal Process. 39, 509 (1991).

[18] Y. Bresler and A. Macovski, IEEE Trans. Acoust. Speech Signal Process. ASSP34, 1081 (1986).

[19] B. de Moor, Linear Algebra and Its Applications 188-189, 163 (1993).

[20] B. de Moor, IEEE Trans. Signal Process. 42, 3104 (1994). 


\section{Appendix B}

\section{Many-Spin FID Simulation}

\section{Package}

\section{B.1 Quantum Spins}

The past few years have brought a rapid increase in the importance of scientific computation. Computer performance has improved tremendously, and powerful new software concepts have been introduced. This is particularly true for many-spin FID calculation, where the absence of exact and general theoretical treatment makes numerical simulation very attractive. Computer simulation of magnetic resonance experiments, however, still requires a rather tedious programming step because standard computer languages do not include many of the data types commonly used to describe the quantum-mechanical spin dynamics. To overcome these deficiencies and 
to assist in the user's implementation, a concise GAMMA program is listed below, which simulates the FID of a polycrystalline dipole-coupled many-spin system under magic-angle spinning. The GAMMA (general approach to magnetic resonance mathematical analysis) library, developed by Ernst group, extends the computer language $\mathrm{C}++$ by adding data type typically used in NMR, such as operators, superoperators, and tensors[1]. This framework enables individual end users to write their particular simulations in an abstract formalism analogous to the analytical description of the problem. None of these programs in this package are particularly long and may be typed-in by hand without too much difficulty.

For systems consisting of just one type of particle, it is sensible to use the reduced units. In this package, the time $t$ is expressed in the unit of

$$
\frac{2 r_{\circ}^{3}}{3 \gamma|\mu|}
$$

where $r_{0}$ is the lattice constant.

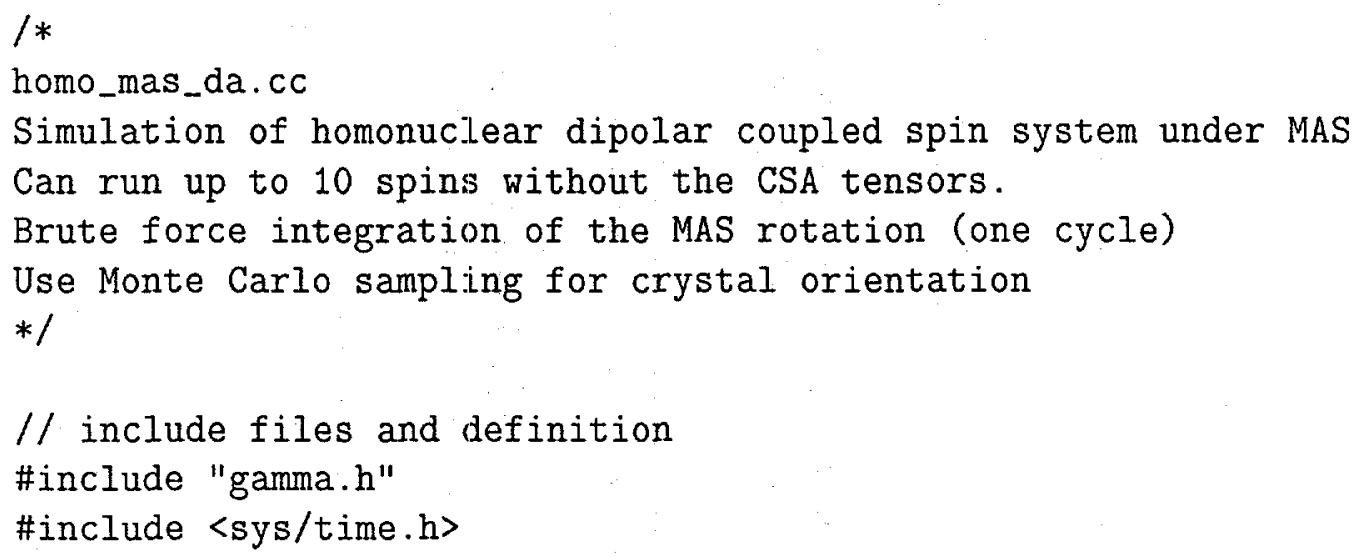


\#include <sys/resource.h>

\#define NPROP 100

\#define MAXSPINS 10

// main program

main(int argc, char *argv[])

\{

// variables declare

spin_system ax(2);

gen_op Ham, U[NPROP], H[5], det, sigma;

spin_T Hdip[MAXSPINS] [MAXSPINS];

space_T Adip [MAXSPINS] [MAXSPINS], Adip_R[MAXSPINS] [MAXSPINS];

double D[MAXSPINS] [MAXSPINS];

int $i, j, k, n f r e e$, nmas, count, detspin;

String name, names;

const double thetam $=54.73561032$;

double dw, scale;

int nspins, index, nprop;

double alpha, beta, gamma;

double alpha_D[MAXSPINS] [MAXSPINS], beta_D [MAXSPINS] [MAXSPINS];

double gamma_D [MAXSPINS] [MAXSPINS] ;

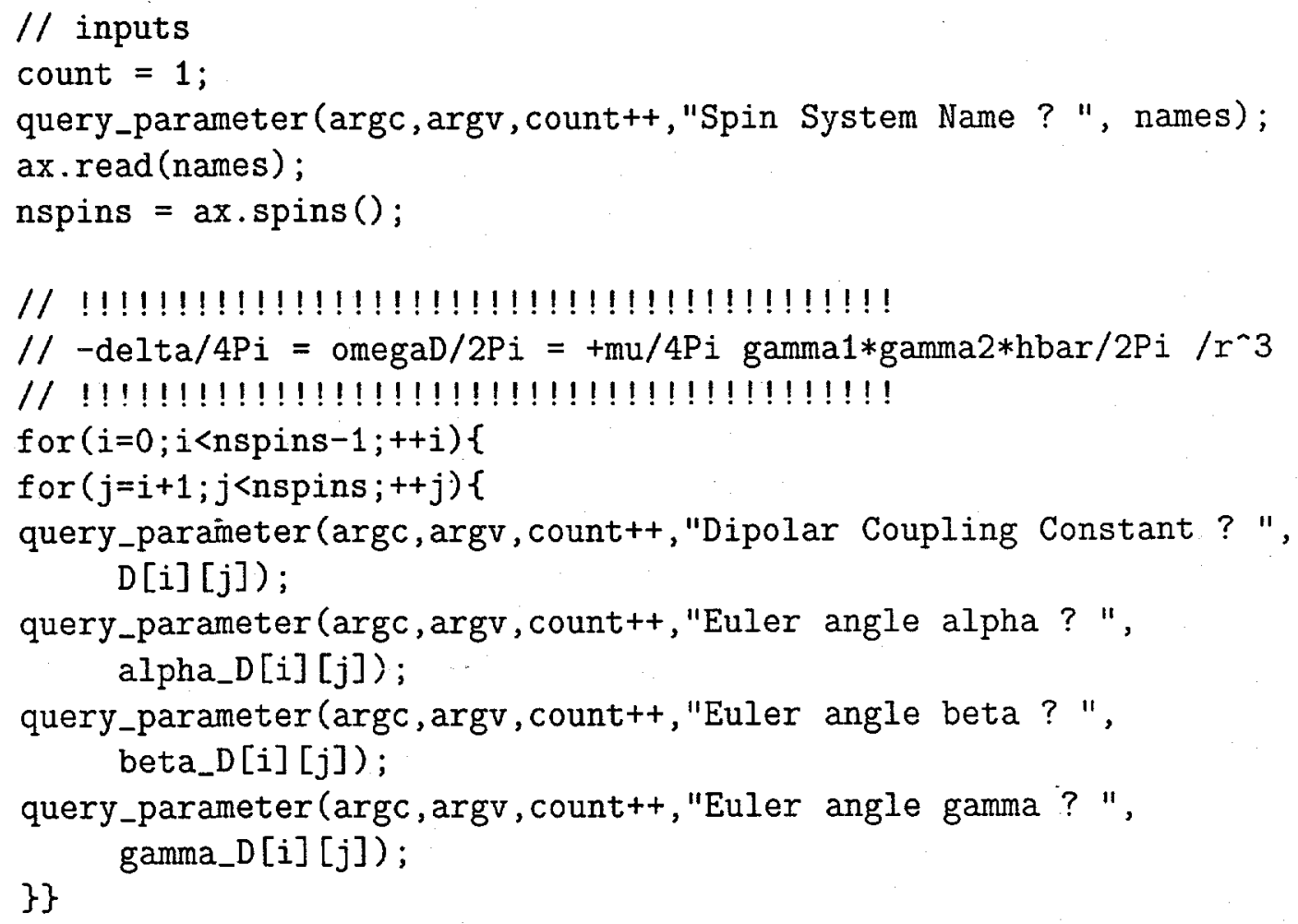


query_parameter (argc, argv, count++, "Dwell Time (sec) ? ", dw);

query_parameter(argc,argv, count++, "\# Points in free FID ?", nfree);

query_parameter (argc,argv, count++,"\# Points in MAS FID ?", nmas);

query_parameter(argc,argv, count++,"\# Points per Rotor Cycle ?", nprop);

query_parameter (argc, argv, count++, "Spin Detected (nth) n=?", detspin);

query_parameter (argc,argv, count++, "Output Filename?", name);

$/ /$ output $\log$

cout $\ll$ "\n\nSimulation of dipolar coupling $\backslash n "$;

cout $<<=============:================\backslash n \backslash n^{\prime \prime}$;

cout $\ll$ "Program version: " $<$ FILE $_{-} \ll$ " compiled at "

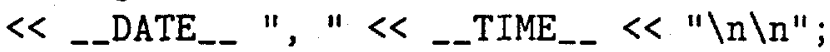

cout $\ll$ "Parameters: \n";

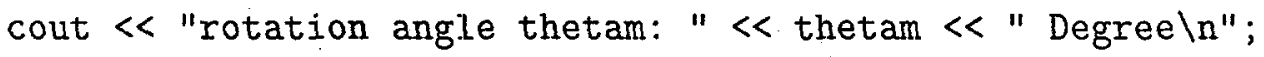

cout < "size of spin system: "< nspins < " spins $\backslash n "$ "

for $(i=0 ; i<$ nspins $-1 ;++i)$

$\{$ for $(j=i+1 ; j<$ nspins $;++j)$

$\{$ cout $\ll$ "dipolar coupling constant (" $<i<$ ", "

$\ll j \ll "$ : : " $<D[i][j] \ll " H z \backslash n "$;

cout $\ll$ "relativ orientation of $D$ tensor: ?"

$\ll$ alpha_D $[i][j] \ll$ "," $\ll$ beta_D $[i][j]$

$\ll "$ " " $\ll$ gamma_D $[i][j] \ll$ " $) \backslash n "$;

\}

\}

cout $\ll$ "Dwell Time (sec): " $<$ dw $\ll$ "\n";

cout $\ll$ "Number of Data Points in free FID: " $<$ nfree $\ll ~ " \backslash n "$;

cout,$<$ "Number of Data Points in MAS FID: " $<$ nmas $\ll ~ " \backslash n "$;

cout $\ll$ "\# of Sampling Points per Rotor Cycle: " $<$ nprop $\ll ~ " \backslash n "$;

cout $\ll$ "Spin Detected (nth) $n=" \ll \operatorname{detspin<<~"\backslash n";~}$

cout $\ll$ "Output filename: " $<$ name $\ll$ " $\backslash n$ ";

cout $\ll$ "\n";

// hamiltonian

for $(i=0 ; i<n s p i n s-1 ;++i)$

$\{$ for $(j=i+1 ; j<$ nspins $;++j)$

$\left\{\operatorname{Hdip}[i][j]=T_{-} D(a x, i, j)\right.$; 


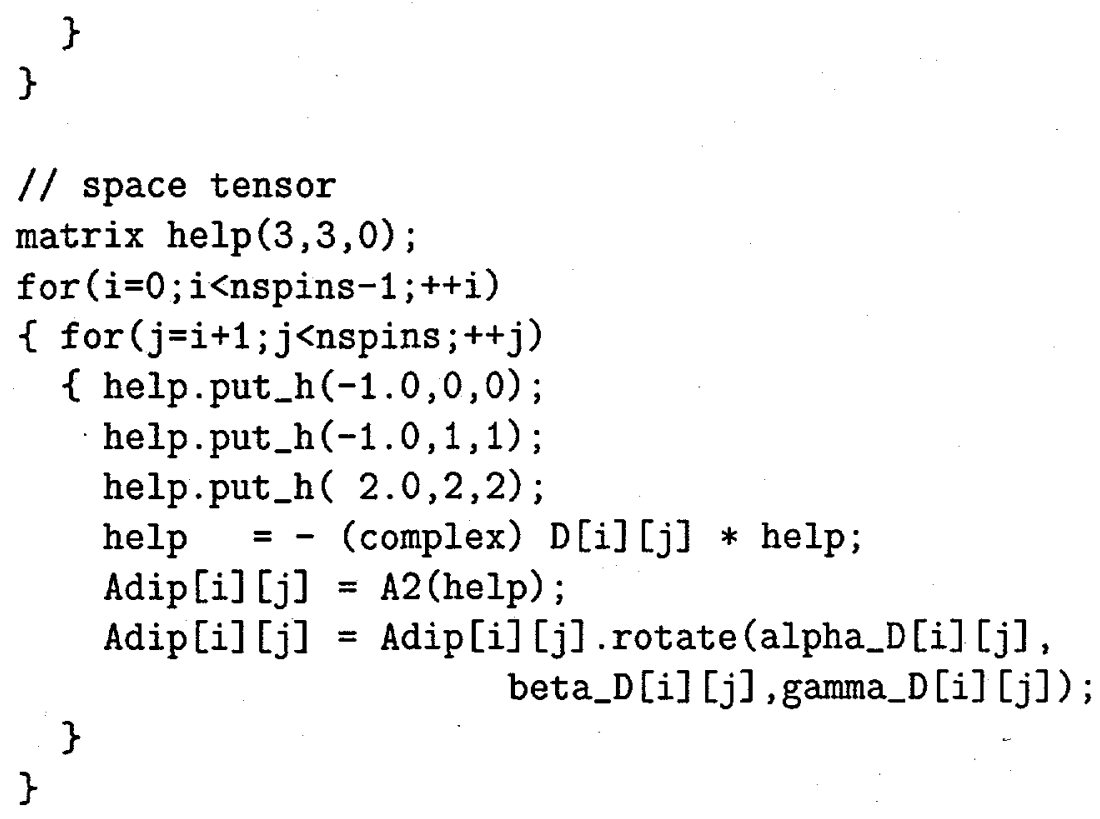


\}

// free FID, nfree pts

Ham $=$ gen_op () ;

if (nfree>0)

$\{$ for $(i=0 ; i<$ nspins $-1 ;++i)$

$\{$ for $(j=i+1 ; j<$ nspins $;++j)$

Ham $+=$ Adip_R $[i][j]$. component $(2,0) * \operatorname{Hdip}[i][j]$. component $(2,0)$;

$/ / \operatorname{Ham}+=\operatorname{ax} \cdot \operatorname{shift}(i) * \operatorname{Iz}(\operatorname{ax}, i)$;

\}

block_1D fidfree(nfree);

String name1 = name+"f.mat";

String name2 = name+" $f$;

FID (sigma, det, Ham, dw , nfree, fidfree);

MATLAB (name1, name 2, fidfree, 1 ) ;

\}

evolve_ip (sigma, Ham,dw*nfree);

// MAS fid, nmas pts, propagator over one cycle of the MAS

if (nmas >0)

$\{$ block_1D fidmas (nmas);

Ham $=$ gen_op () ;

for (index $=0$; index<nprop; ++index)

$\{$ for $(i=-2 ; i<=2 ;++i)$

Ham $+=\exp (\operatorname{complex}(0, i * 2.0 * P I * i n d e x /$ double (nprop) $)) * H[i+2]$;

$U[$ index $]=\operatorname{prop}(\mathrm{Ham}, \mathrm{dw})$;

\}

for $(i=0 ; i<$ nprop; $++i)$

$\mathrm{U}[\mathrm{i}]$. set_DBR();

$\operatorname{det}$.set_DBR();

sigma.set_DBR();

for $(i=0 ; i<$ nmas $;++i)$

$\{$ fidmas $(i)+=$ trace (det, sigma)*scale;

sigma.sim_trans_ip(U[i\%nprop]);

\}

String name1 = name+"m.mat";

String name2 = name+" $\mathrm{m}$ ";

MATLAB (name1, name2, fidmas ,1);

\}

\} 


\section{B.2 Classical Spins}

Table B.1: Summary of Subroutines for Classical Spin Simulations

\begin{tabular}{|c|c|}
\hline Program & Description \\
\hline $\begin{array}{l}\text { spinmd.m } \\
\text { spinmd_sig.m } \\
\text { spinmd_bloc.m } \\
\text { spinmd_dot.m } \\
\text { spinmd_crd.m } \\
\text { spinmd_ed.m } \\
\text { spinmd_ndx.m } \\
\text { spinmd_roty.m } \\
\text { spinmd_avg.m }\end{array}$ & $\begin{array}{l}\text { main program } \\
\text { dipolar Hamiltonian tensor } \\
\text { dipolar local fields } \\
\text { evolution under local fields } \\
\text { simple-cubic lattice coordinates } \\
\text { dipolar energy } \\
\text { periodic boundary condition } \\
\text { pulse rotation along } y \text { axis } \\
\text { canonical ensemble average }\end{array}$ \\
\hline
\end{tabular}

A genuine numerical approach to calculate the FIDs would require diagonalization of matrices of dimension $(2 I+1)^{N}$, where $I$ is the spin quantum number and $N$ is the number of coupled spins in the system. This would create a hopeless situation for $N$ sufficiently large to faithfully bring out the many-spin effects. In the classical limit, however,

$$
I \rightarrow \infty, \hbar \rightarrow 0, \gamma \hbar \sqrt{I(I+1)} \rightarrow|\mu|,
$$

( $\gamma$ : gyromagnetic ratio, $\boldsymbol{\mu}$ : magnetic moment) and the dimension greatly reduces to $2 N$ : two polar angles for each classical spin. Such computationally feasible classicalspin simulations can then be used to verify the validity of theoretical predictions 
in the limit of $I \rightarrow \infty$. One can also monitor a single trajectory, where as a real experiment can only provide ensemble average of all the trajectories.

The main program is listed below. Its subroutines and computational procedures are summarized in Table B.1.

$\%$ spinmd.m

$\%$ spinmd: molecular dynamics simulations for classical spins

$\%$ main program:

$\%$ FIDs of observed spins in dissipatice simple-cubic lattice

$\%$ subject to many-spin homonuclear dipole-dipole interaction

$\%===$ simulation parameters

$\%$ number of trajectories

mcno=10000;

$\%$ length of the simple-cubic lattice $=2 * \mathrm{RG} 1+1$

RG1=2;

$\%$ radius of interaction sphere

$\mathrm{RG} 2=2$;

$\%$ radius of observed sphere (the "spins")

RG3=2;

$\%$ initial seed for random number generator

seed=0;

$\%$ dipolar Hamiltonian: =0, truncated; =1, complete ham $=0$;

$\% g=[g x g v g z]$, strength of the spin-lattice coupling.

$\%$ for normal dipolar interaction, $g=\left[\begin{array}{lll}1 & 1 & 1\end{array}\right]$

$\mathrm{g}=\left[\begin{array}{lll}1 & 1 & 1\end{array}\right]$;

$\%$ structure of config: [randseed,hx,hy,hz,Ez,Ed] where

$\% \mathrm{hx}, \mathrm{hy}, \mathrm{hz}$ are the dipolar local-fields acting on the tagged spin

$\%$ after the pulse and Ez (Ed) is the total Zeeman (dipolar)

$\%$ energy of the spintlattice system

config=zeros $($ mcno, 6$)$;

$\%===$ central cell

$\%$ crd1, e0, et, $\mathrm{H}$ share the same index rule and structure

$\% \mathrm{ndx}=(\operatorname{crd} 1(:, 1)+\mathrm{RG} 1): *(2 * \mathrm{RG} 1+1)^{\wedge} 2+$ 
$\%(\operatorname{crd} 1(:, 2)+\mathrm{RG} 1) *(2 * \mathrm{RG} 1+1)+(\operatorname{crd} 1(:, 3)+\mathrm{RG} 1)+1$

$\%$ crd1: absolute coordinates [ $x$ y $z$ a] of the

$\%$ central lattice containing $\mathrm{N} 1=(-\mathrm{RG} 1: \mathrm{RG} 1)-3$ spins

$\%$ e0, et: [e1x e1y e1z;...;eN1x en1y eN1z]

$\% \mathrm{H}:$ [H1x H1y H1z; ..; HN1x HN1y HN1z]

[crd1, N1] =spinmd_crd (RG1);

$\%===$ interaction sphere

$\%$ local field: consider only spins within a sphere of radius $\mathrm{RG} 2$

$\% \mathrm{~N} 2$ : number of neighbors within the sphere

$\%$ structure of crd2: [ $\left.\begin{array}{lll}x & y & a\end{array}\right], a=r / r 0$

$[\mathrm{crd} 2, \mathrm{~N} 2]=$ spinmd_rad $(\mathrm{RG} 2)$;

$\%===$ observed sphere

[crd3, N3] =spinmd_rad (RG3);

$\%$ index of the observed spins

$\mathrm{ndx} 0=$ spinmd_ndx $(\operatorname{crd} 3, \mathrm{RG} 1)$;

$\%===$ sigma: spatial part of the dipolar Hamiltonian

sigma=spinmd_sig(crd2,ham_ic);

$\%===$ initial configurations

rand ('seed', seed);

for $i i=1: m c n o$

config ( $i i, 1)=$ rand ('seed');

$\%$ e 0 et: size $\mathrm{N} 1 * 3$

e $0=$ spinmd_ic (N1, config $(i i, 1), 1)$;

$\%$ fixed the observed spins at $\left[\begin{array}{lll}1 & 0 & 0\end{array}\right]$

e $0(\operatorname{ndx} 0,:)=[$ ones $(N 3,1) \operatorname{zeros}(N 3,2)]$;

$\%$ local fields acting on the observed spins

$n d x=$ spinmd_ndx $(\operatorname{crd} 2(:, 1: 3), R G 1)$;

tagged $=1$;

config (ii, 2:4)=spinmd_bloc (sigma, e0, ndx, ndx0, tagged, g);

$\%$ total Zeeman Energy

config (ii, 5) $=-\operatorname{sum}(e 0(:, 3))$;

$\%$ total dipole Energy

[Edtotal ,Ed] =spinmd_ed (e0, RG1, crd1, crd2 , sigma, ndx0, g);

config $(i \dot{i}, 6)=$ Edtotal;

end $\% \mathrm{mcno}$ 
$\%===$ evolution

ham_evo $=0$;

fileno $=[0: 24]$;

iterno $=400$;

tspan $=[0: 0.02: 3.5]$. '; $^{\circ}$

options=odeset ('RelTol' , 1e-2);

$\%$ reaction field: $=0$ without; $=1$ with;

ifrf $=1$;

$\%===$ dipolar Hamiltonian

sigma=spinmd_sig (crd2, ham_evo);

for filecnt=1:length (fileno)

filecnt

et=zeros (length (tspan) , $3 * \mathrm{~N} 1)$;

mux $=$ zeros (iterno, length (tspan));

muy $=\operatorname{zeros}$ (iterno, length (tspan));

muz=zeros (iterno, length (tspan));

for itercnt=1:iterno

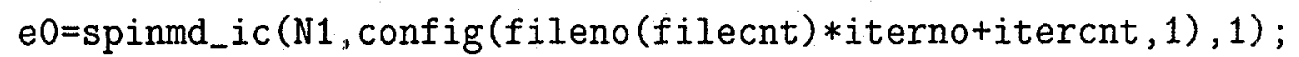

$e 0(n d x 0,:)=\left[\begin{array}{lll}1 & 0 & 0\end{array}\right]$;

$\%===$ evolution in the rotating frame

$\%$ change e0's structure to: [e1x;e1y;e1z;... ;eN1x;eN1y;eN1z]

$[t, e t]=$ ode45 ('spinmd_dot', tspan, reshape $(e 0$. ', $3 * N 1,1)$, options , . .

crd1, N1, RG1, crd2, sigma, ndx0, g, ifrf);

$\operatorname{mux}($ itercnt,$:)=$ et $(:, 3 *($ ndx $0-1)+1) .^{\prime}$;

muy (itercnt, $:)=e t(:, 3 *($ ndx $0-1)+2) .{ }^{2}$;

muz (itercnt, : ) $=\operatorname{et}(:, 3 *(\mathrm{ndx} 0-1)+3) .^{\prime}$;

$\%$ save file

if rem(itercnt, 50) $==0$

[filecnt itercnt]

eval(['save ind1_' fn num2str(fileno(filecnt)) ...

'. mat mux muy muz tspan itercnt ham_evo ifrf']);

end

end $\%$ iterno

end $\%$ fileno 


\section{B.2.1 Dipole-Dipole Interaction}

For a system of $N$ identical classical spins in a rigid lattice subject to a large external Zeeman field $B_{\circ}$ along the $z$ axis, the effective interaction in the rotating frame precessing with the Larmor frequency $\omega_{0}=-\gamma B_{0}$ can be approximated by the truncated dipolar Hamiltonian $\mathcal{H}_{d}^{\circ}$ (spinmd_sig.m)

$$
\begin{gathered}
\mathcal{H}_{d}^{\circ}=\sum_{i=0}^{N-1} \sum_{j>i} \mathcal{H}_{d, i j}^{\circ} \\
\mathcal{H}_{d, i j}^{\circ}=D_{i j}\left(2 \mu_{z i} \mu_{z j}-\mu_{x i} \mu_{x j}-\mu_{y i} \mu_{y j}\right) \\
D_{i j}=\frac{1-3 \cos ^{2} \theta_{i j}}{2 r_{i j}^{3}}
\end{gathered}
$$

where $r$ is the internuclear distance, and $\theta$ is the angle between the internuclear vector and the Zeeman field. The presence of a strong Zeeman field serves to identify the $z$ component of magnetization with a large Zeeman energy, and the high-field truncation is designed such that only motions conserving this energy are allowed[2].

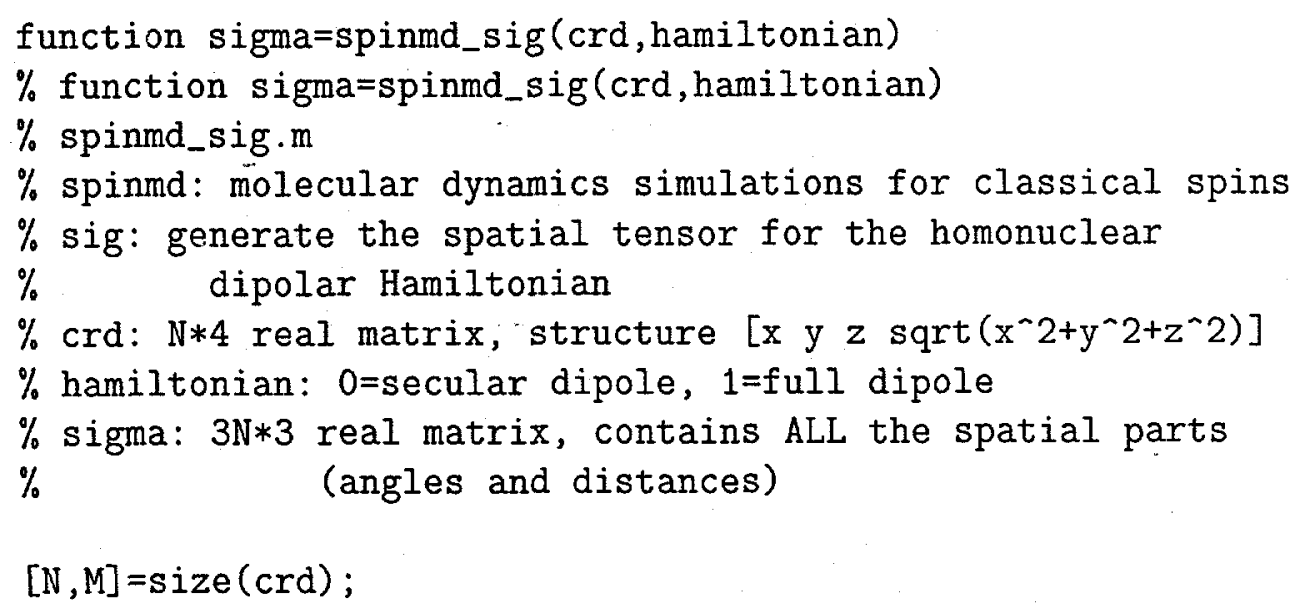




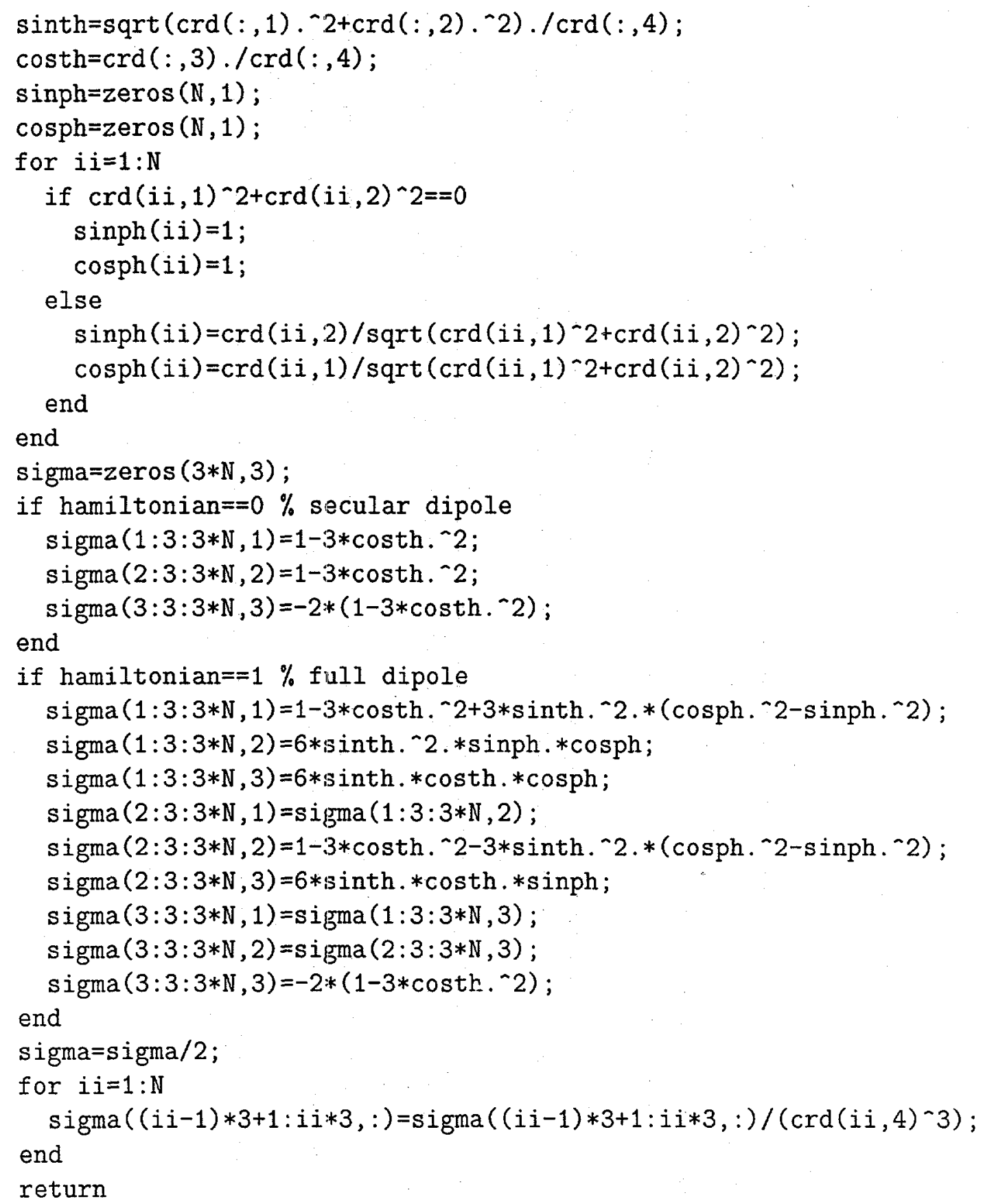




\section{B.2.2 Dipolar Local Fields}

Rewrite the interaction in the one-spin picture for an arbitrary spin labeled with subscript "0",

$$
\mathcal{H}_{d}^{\circ}=-\boldsymbol{h}_{0} \cdot \boldsymbol{\mu}_{0}+\sum_{i=1}^{N-1} \sum_{j>i} \mathcal{H}_{d, i j}^{\circ}
$$

where $h_{0}=\left[h_{x 0}, h_{y 0}, h_{z 0}\right]^{T}$ are the dipolar local fields acting on the tagged spin produced by its neighbors, $\mu_{0}=\left[\mu_{x 0}, \mu_{y 0}, \mu_{z 0}\right]^{T}$, and

$$
\begin{gathered}
h_{z 0} \equiv-2 \sum_{i=1}^{N-1} D_{0 i} \mu_{z i}, \\
h_{\alpha 0} \equiv \sum_{i=1}^{N-1} D_{0 i} \mu_{\alpha i}(\alpha=x, y) .
\end{gathered}
$$

function bloc=spinmd_bloc (sigma, et, ndx, ndx0, tagged, $g$, ifrf)

$\%$ function bloc=spinmd_bloc (sigma, et, ndx, ndx 0, tagged,g, ifrf)

$\%$ spinmd_bloc.m

$\%$ spinmd: molecular dynamics simulations for classical spins

$\%$ bloc: calculate dipolar local fields

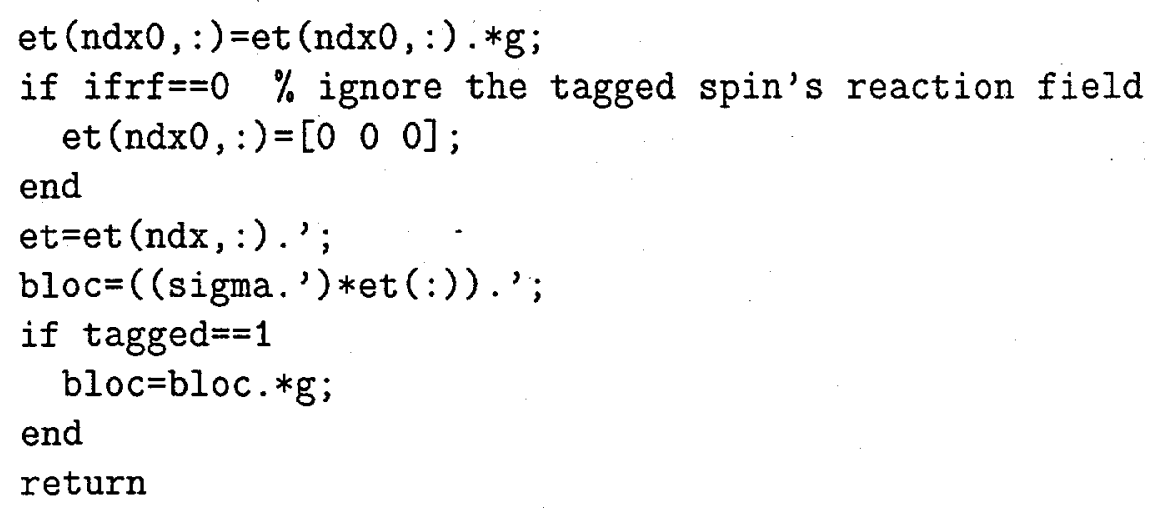




\section{B.2.3 Equation of Motion}

Each and every spin precesses under the torque exerted by its local fields, as described by the set of differential equations

$$
\frac{d}{d t} \mu_{i}(t)=\gamma \mu_{i}(t) \times h_{i}(t)(i=0, \cdots, N-1)
$$

The time evolution of all the magnetic moments $\left\{\boldsymbol{\mu}_{i}(t)\right\}_{(i=0, \cdots, N-1)}$ is then numerically solved by integrating the system of differential equations, Eq (B.9), based on explicit Runge-Kutta (4,5) algorithm of Dormand-Prince [4].

function edot=spinmd_dot ( $t$, et,flag, crd1,N1,RG1, crd2,

$$
\text { sigma, ndx } 0, g, \text { ifrf) }
$$

$\%$ function edot=spinmd_dot $(t$, et,flag, $, \operatorname{crd} 1, N 1, R G 1$,

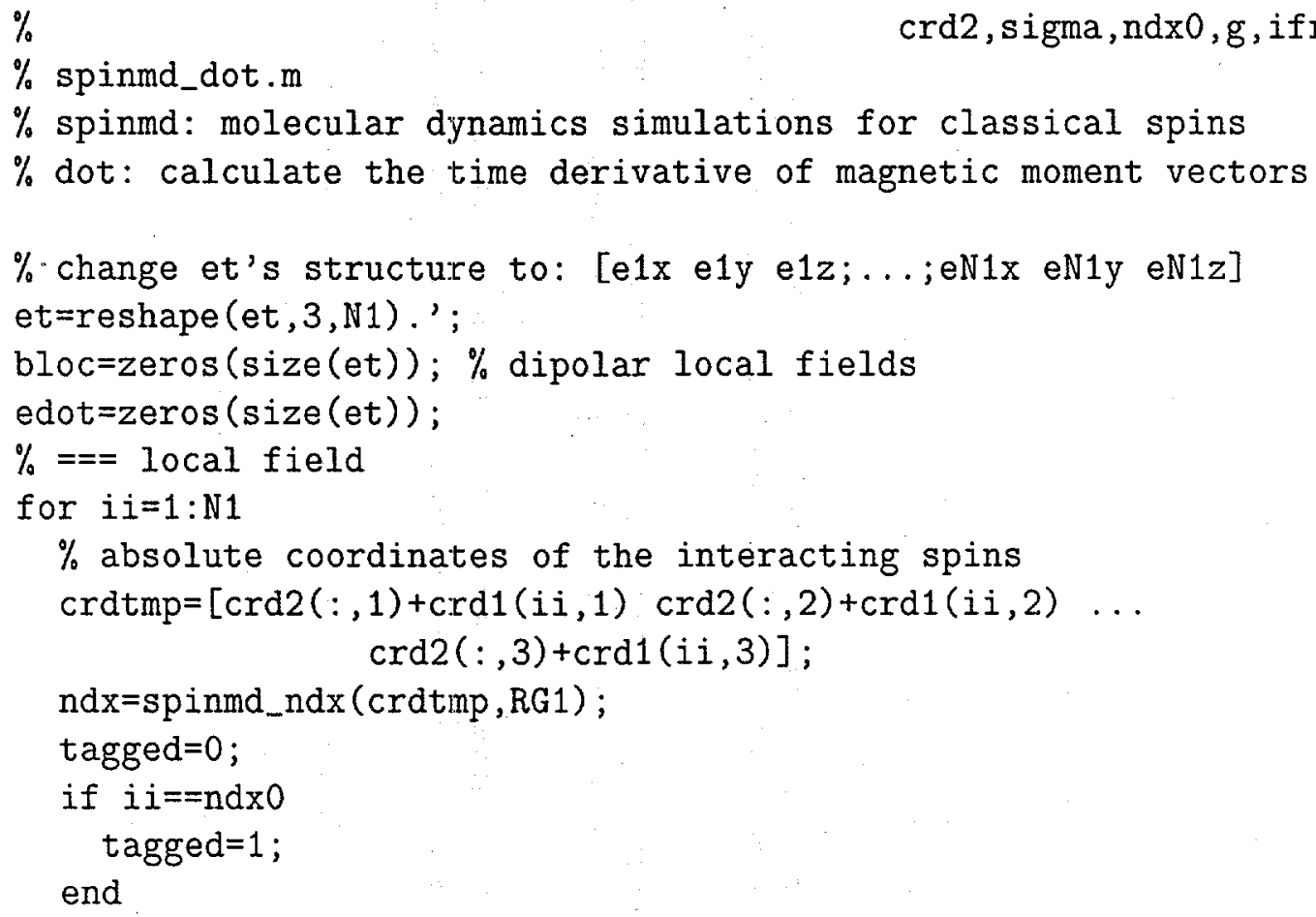




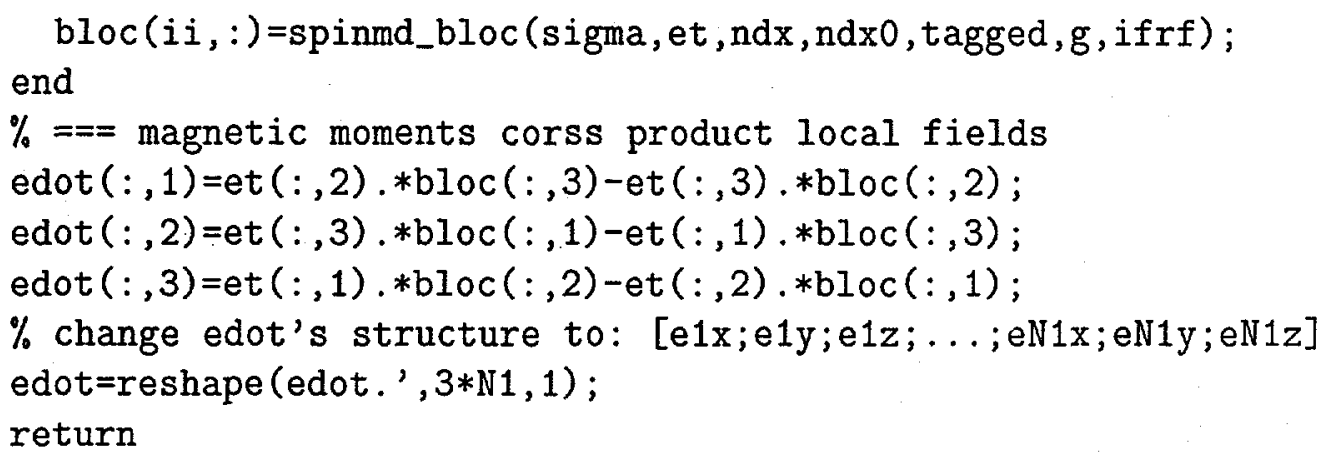

\section{B.2.4 Initial Configurations}

The initial configuration $\left\{\boldsymbol{\mu}_{i}(0)\right\}_{(i=0, \cdots, N-1)}$ is obtained by generating $N$ uniformly distributed random vectors on the surface of a sphere with radius $|\boldsymbol{\mu}|[3]$. The associated total dipolar energy $\mathcal{H}_{d}^{\circ}$ and total Zeeman energy $\mathcal{H}_{\circ}$ are calculated respectively by Eqs. (B.3)-(B.5) (spinmd_ed.m) and

$$
\mathcal{H}_{\circ}=-B_{\circ} \sum_{i=0}^{N-1} \mu_{z i}
$$

function $[\mathrm{crd} ; \mathrm{N}]=$ spinmd_crd $(r)$

$\%$ spinmd_crd.m

$\%$ spinmd: molecular dynamics simulations for classical spins

$\%$ crd: generate coordinates of the integer points

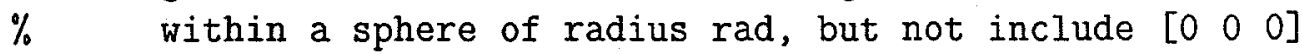

$\% r: 1 * 1$ integer, helf-length of the simple-cubic lattice

$\%$ structure of crd: [x y z sqrt $\left.\left(x^{\wedge} 2+y^{\wedge} 2+z^{\wedge} 2\right)\right]$

$\%$ crd has the following index rule and structure

$\% \mathrm{ndx}=(\operatorname{crd}(:, 1)+\mathrm{r}) *(2 * \mathrm{r}+1)-2+$

$\% \quad(\operatorname{crd}(:, 2)+r) *(2 * r+1)+(\operatorname{crd}(:, 3)+r)+1$

$\%$ crd: coordinates $\left[x\right.$ y $z$ sqrt $\left.\left(x^{\wedge} 2+y^{\wedge} 2+z^{\wedge} 2\right)\right]$ of the

$\%$ central lattice containing $N=(-r: r)-3$ spins 


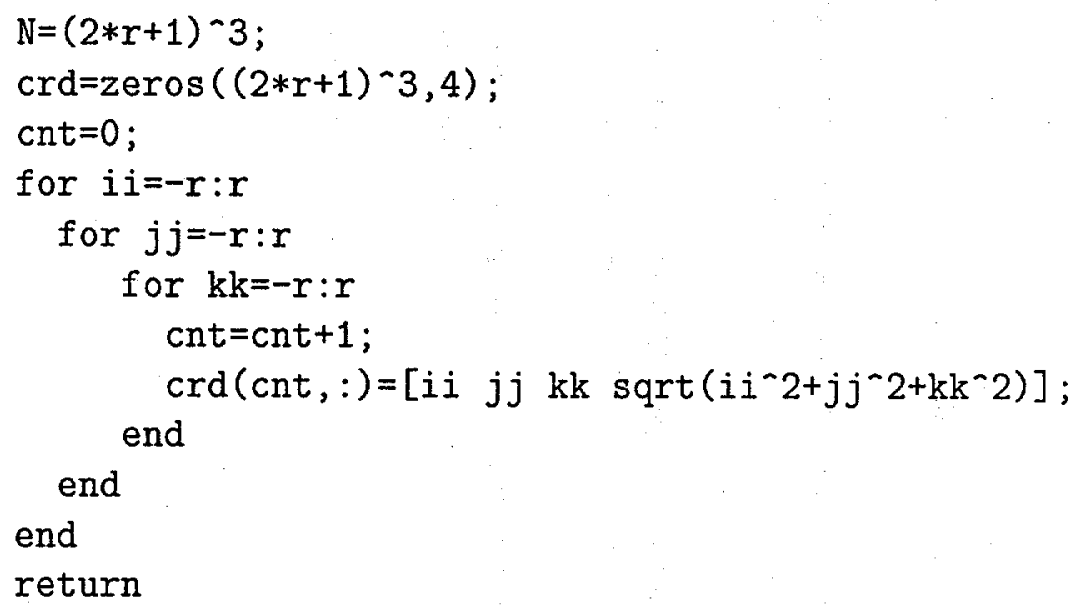

function [Edtotal, Ed] =spinmd_ed (e0,RG1, crd1, crd2, sigma, ndx0,g)

$\%$ function [Edtotal, Ed] =spinmd_ed $(e 0, \operatorname{RG} 1, \operatorname{crd} 1, \operatorname{crd} 2$, sigma, ndx $0, \mathrm{~g})$

$\%$ spinmd_ed.m

$\%$ spinmd: molecular dynamics simulations for classical spins

$\%$ ed: calculate the dipolar energies for that specific configuration

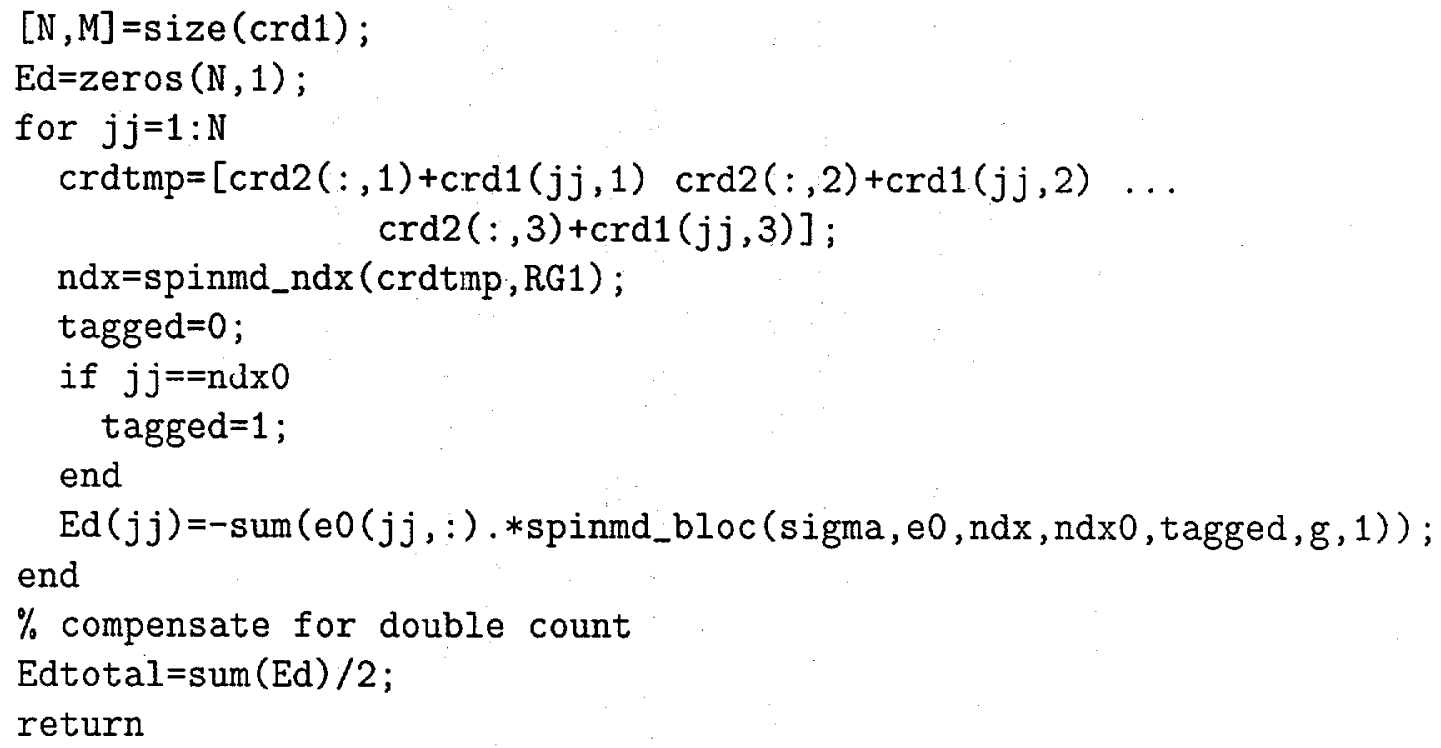


$\%$ function e0=spinmd_ic $(\mathrm{N}$, randseed $)$

$\%$ spinmd_ic.m

$\%$ spinmd: molecular dynamics simulations for classical spins

$\%$ ic: generate $\mathrm{N}$ uniformly distributed random vectors on the

$\% \quad$ surface of a unit sphere

\% refs: G. Marsaglia, Ann. Math. Stat. 43, 645 (1972).

rand ('seed', randseed);

eta $=\operatorname{zeros}(\mathrm{N} * 10,3)$;

eta $(:, 1: 2)=1-2 *$ rand $(\mathrm{N} * 10,2)$;

$\operatorname{eta}(:, 3)=\operatorname{eta}(:, 1) \cdot \wedge^{\wedge} 2+e t a(:, 2) \cdot \wedge 2$;

tmp $=e t a(f i n d(e t a(:, 3)<1),:)$;

$\%$ e0: size $\mathrm{N} * 3$

$\mathrm{e} 0=[2 * \operatorname{tmp}(1: \mathrm{N}, 1) . * \operatorname{sqrt}(1-\operatorname{tmp}(1: \mathrm{N}, 3)), \ldots$

$2 * \operatorname{tmp}(1: N, 2) . * \operatorname{sqrt}(1-\operatorname{tmp}(1: N, 3)), 1-2 * \operatorname{tmp}(1: N, 3)]$;

return

function $n d x=$ spinmd_ndx (crdtmp, RG1)

$\%$ function $n d x=$ spinmd_ndx (crdtmp, RG1)

$\%$ spinmd_ndx.m

$\%$ spinmd: molecular dynamics simulations for classical spins $\%$ ndx: periodic boundry condition

$\%===$ periodic $\mathrm{BC}$

crdtmp $=\operatorname{crdtmp}-$ round $(\operatorname{crdtmp} /(2 * \mathrm{RG} 1+1)) *(2 * \mathrm{RG} 1+1)$;

$\% \mathrm{ndx}$ : index of spins, map to crd1

$\mathrm{ndx}=((\operatorname{crdtmp}(:, 1)+\mathrm{RG} 1) *(2 * \mathrm{RG} 1+1)-2+\ldots$

$(\operatorname{crdtmp}(:, 2)+\mathrm{RG} 1) *(2 * \mathrm{RG} 1+1)+(\operatorname{crdtmp}(:, 3)+\mathrm{RG} 1)+1) ;$

return

function [crd, N] =spinmd_rad (rad)

$\%$ function [crd, N] = spinmd_rad (rad)

$\%$ spinmd_rad.m

\% spinmd: molecular dynamics simulations for classical spins

$\%$ rad: generate coordinates of the integer points

$\% \quad$ within a sphere of radius rad, but not include $\left[\begin{array}{lll}0 & 0 & 0\end{array}\right]$ 
$\%$ rad: $1 * 1$ integer, radius of the sphere

$\% \mathrm{~N}$ : number of neighbors within the sphere $\mathrm{N}=0$;

for $i i=-f l o o r(r a d): c e i l(r a d)$

for $j j=-f l o o r(\mathrm{rad}):$ ceil (rad)

for $k k=-f l o o r(r a d): c e i l(r a d)$

if $i i^{\wedge} 2+j j^{\wedge} 2+k k^{\wedge} 2<=\operatorname{rad}^{\wedge} 2 \& i i^{\wedge} 2+j j^{\wedge} 2+k k^{\wedge} 2>0$ $\mathrm{N}=\mathrm{N}+1$; end

end

end

end

$\%$ structure of crd: $\left[x\right.$ y $\left.z \operatorname{sqrt}\left(x^{\wedge} 2+y^{\wedge} 2+z^{\wedge} 2\right)\right]$

$\operatorname{crd}=\operatorname{zeros}(\mathrm{N}, 4)$;

cnt $=0$;

for $i i=-f l o o r(\mathrm{rad}): c e i l(\mathrm{rad})$

for $j j=-f l o o r(\mathrm{rad})$ : ceil (rad)

for $k k=-f l o o r(r a d): c e i l(r a d)$

if $i i^{\wedge} 2+j j^{\wedge} 2+k k^{\wedge} 2<=\operatorname{rad}^{\wedge} 2$ \& $i i^{\wedge} 2+j j^{\wedge} 2+k k^{\wedge} 2>0$

cnt $=$ cnt +1 ;

$\operatorname{crd}(\operatorname{cnt},:)=\left[i i j j k k \operatorname{sqrt}\left(i i^{\sim} 2+j^{\wedge} j^{\sim} 2+k k^{\wedge} 2\right)\right]$; end

end

end

end

return

\section{B.2.5 Pulse Rotation}

All the spins are first treated by an ideal pulse along $-y$-axis with tipping angle

$\theta$. The corresponding rotation in the spin space is

$$
\mu_{j}\left(0_{+}\right)=\left[\begin{array}{ccc}
\cos (\theta) & 0 & -\sin (\theta) \\
0 & 1 & 0 \\
\sin (\theta) & 0 & \cos (\theta)
\end{array}\right] \mu_{j}(0), \text { for } j=0, \cdots, N-1
$$


where time $t=0_{+}$denotes the point immediately after the pulse.

function e0=spinmd_roty (e0,theta)

$\%$ function e0=spinmd_roty (e0, theta)

$\%$ spinmd_roty.m

$\%$ spinmd: molecular dynamics simulations for classical spins

$\%$ roty: rotate vector along $+y$ axis by theta angle (in radius)

$\%$ rotation matrix $[\cos ($ theta) $0-\sin ($ theta) $; 010$;

$\% \quad \sin ($ theta) $0 \cos ($ theta) $]$

$\mathrm{e} 0=([\cos ($ theta $) 0-\sin ($ theta $) ; 010 ; \ldots$

$\sin ($ theta) $0 \cos ($ theta) $] * e 0 . ') . '$;

return

\section{B.2.6 Canonical Ensemble Average}

Finally, the FID is estimated by averaging $\sum_{j=0}^{N-1} \mu_{x j}(t)$ over the trajectories weighed by the equilibrium Boltzman probabilities of the initial configurations

$$
\left\langle\sum_{j=0}^{N-1} \mu_{x j}(t)\right\rangle \propto \sum_{\text {trajectories }} \mathrm{e}^{-\beta\left(\mathcal{H}_{\circ}+\mathcal{H}_{d}^{\circ}\right)} \sum_{j=0}^{N-1} \mu_{x j}(t)
$$

function $[f i d 0, f i d 1]=\operatorname{spinmd} \_a v g(M, b e t a, E s, E I, E s I)$

$\%$ function [fido,fid1]=spinmd_avg(M, beta,Es,El,EsI)

$\%$ spinmd_sl.m

$\%$ spinmd: molecular dynamics simulations for classical spins

$\%$ avg: canonical ensemble average

$\% \mathrm{M}$ : magnetization data, (\# trajectories)*(\# fid points)

$\%$ Es: spin energy, (\# trajectories) $* 1$

$\% E l$ : lattice energy, (\# trajectories) $* 1$

$\%$ Esl: spin-lattice coupling energy

$\%$ fido $=\exp [-b(E s+E 1)] * M$ 
$\%$ fid1 $=\{[\exp (-\mathrm{b} * \mathrm{Es} 1)-1] * \exp [-\mathrm{b} *(\mathrm{Es}+\mathrm{E} 1)]\} * \mathrm{M}$

fido $=(\exp (-\operatorname{beta} *(E \mathbf{s}+E 1)) . ') * M$;

$\%$ fid1 $=(((\exp (-$ beta $* E s 1)-1) \cdot * \exp (-\operatorname{beta} *(E s+E 1))) . ') * M$;

fid1 $=((-$ beta $*$ Esl.$* \exp (-$ beta $*(E s+E 1))) . ') * M$;

return 


\section{Bibliography}

[1] S. A. Smith, T. O. Levante, B. H. Meier, and R. R. Ernst, J. Magn. Reson. A 106, 75 (1994).

[2] A. Abragam, Principles of Nuclear Magnetism (Clarendon, Oxford, 1961).

[3] G. Marsaglia, Ann. Math. Stat. 43, 645 (1972).

[4] J. R. Dormand and P. J. Prince, J. Comp. Appl. Math. 6, 19 (1980). 